\title{
A home away from home? An interpretative phenomenological analysis of Vietnamese students' acculturation experiences in Aotearoa-New Zealand
}

\author{
By \\ Hau Trung Ho \\ A thesis \\ submitted to Victoria University of Wellington \\ in fulfilment of the requirements for the degree of \\ Doctor of Philosophy
}





\section{Abstract}

International students' experiences are a major concern for universities and educational researchers. Globally, Vietnam is a top source country for international students. Universities in Aotearoa-New Zealand host an increasing number of Vietnamese students, but researchers often subsume this group into generic cultural and ethnic categories such as 'Asian student'. As a result, little is known about their experience.

This study explores the everyday experiences of 10 Vietnamese master's students from an Aotearoa-New Zealand university through a series of semi-structured in-depth interviews. An interpretative phenomenological analysis approach enabled me to capture the essential meanings of the participants' experiences and understand what it was like to be Vietnamese students in Aotearoa-New Zealand.

As the participants built new lives in Aotearoa-New Zealand, they dealt with the practicalities of uncomfortable or difficult housing conditions as well as established relationships within the diverse cultural contexts of the host country. Accommodation issues had a significant impact on their studies and sense of security and belonging. The students had a hard time finding a place to live. Not feeling comfortable, safe or at peace in their new houses, they did not feel like they were at home. They did not view their houses as a place where they could take refuge and leave the hustle of life outside.

In academic settings, the students initially struggled to deal with a range of new and unfamiliar learning and teaching styles (e.g., classroom discussion) and conventions about referencing and citing. These made them feel overwhelmed and bewildered. During these formative months, many of them experienced language difficulties, a sense of cultural dislocation, and a deep yearning for their families. These difficulties forced them to learn to deal with challenges and become independent. The students drew strength from their Vietnamese cultural values and practices to overcome difficulties.

Based on the findings, I propose a framework for exploring the experience of short-term Vietnamese international students. The study provides implications for host universities to assist Vietnamese students as they orient themselves to daily life in Aotearoa-New Zealand. 


\section{Acknowledgements}

Undertaking a PhD is a transformative experience. Getting to this point has been a long journey with many individuals assisting me along the way. With deep gratitude to Dr Stephanie Doyle and Professor Joanna Kidman. It was my very good fortune to have them as my supervisors. Words are not enough to express how grateful I am for all that both have done for me. Their wisdom, knowledge, and encouragement have inspired me to persevere and work harder. Joanna's constructive and insightful feedback made an invaluable contribution to the quality of my project. Special thanks to Stephanie for guiding me through stages of my doctoral studies and for always being there when needed. The four-and-a-half-year experience of working with her was enjoyable and memorable.

To the participants for their time, trust, openness, and commitment in sharing their experiences. Their stories are the soul of this study. Without their contribution, my study would not have been possible. I wish them all the best for the next phase of their lives.

To the examiners, Dr Carolyn Tait, Professor Ly Tran, and Associate Professor Jennie Billot, for their very thoughtful and insightful feedback which helped improve my thesis significantly. I highly appreciated their deep engagement with my thesis.

To doctoral students at 3 Waiteata Road and those in the cohort group, who considerably contributed to my doctoral experiences. I appreciated the knowledge they shared with me.

To Dr Lizzie Towl and Dr Sara Cotteral for organising fantastic workshops, where I learnt a lot. I would like to acknowledge Lizzie for running the Thesis Bootcamp, which provided me with an opportunity to make remarkable progress on my thesis.

To Minh Tran for being understanding and supportive. Discussion with her gave me new insights into academic issues. Her fellowship and encouragement helped me overcome the challenges of undertaking my PhD.

Last but not least, to my parents and sisters who have always believed in me and encouraged me in all my pursuits. They have been present in all stages of my life and always want the best for me. My parents' unconditional love and quiet care have given me the strength to get through the tough times. I would not be who I am without their unparalleled support. After a journey of a thousand miles, I know that I have a home to return and rest. I am forever indebted to my parents for all they have done for me. 


\section{Publication from this Research}

Ho, T. H. (2020). The experiences of Vietnamese students in New Zealand: A new country - a new home. Transitions: Journal of Transient Migration, 4(2), 203-221.

\section{Conference Presentations}

Ho, T. H. (2019, July). From cosy home to the outside world: Just a sightseeing? Vietnamese international students in New Zealand. Paper presented at the European Conference on Education, London, UK.

Ho, T. H. (2019, December). The experiences of Vietnamese students in New Zealand: A phenomenological study. Paper presented at the New Zealand Association for Research in Education (NZARE), Christchurch, New Zealand. 


\title{
Table of Contents
}

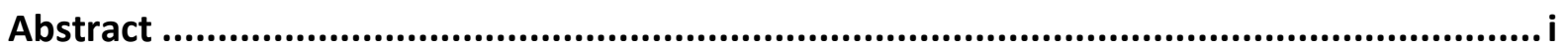

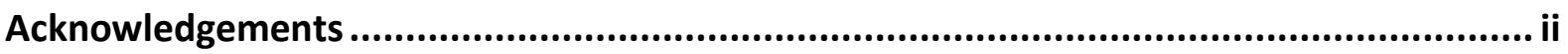

Publication from this Research .......................................................................................ii

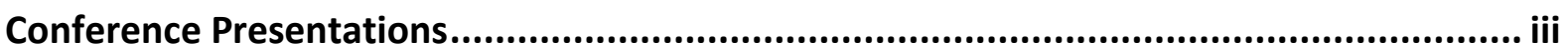

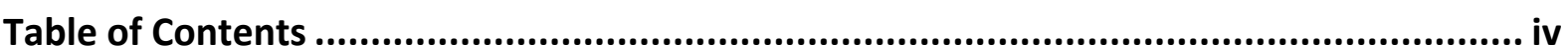

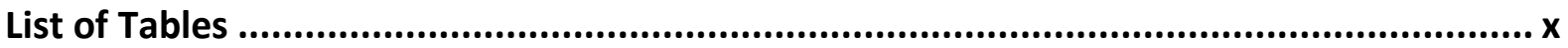

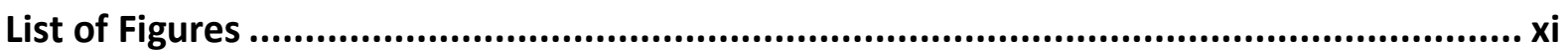

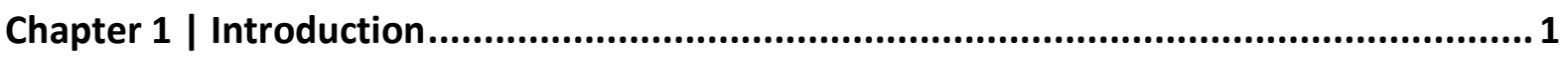

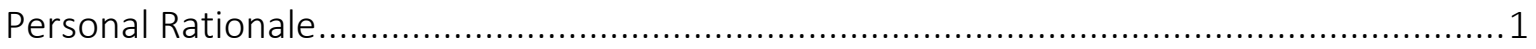

Aotearoa-New Zealand's Role in International Education ..............................................

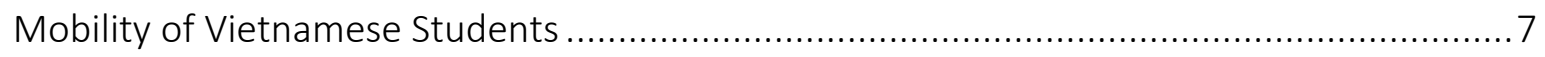

Teaching and Learning in the Vietnamese Higher Education ........................................10

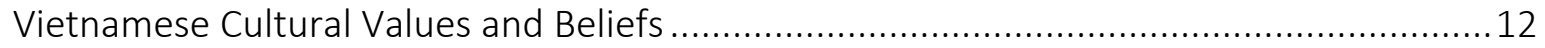

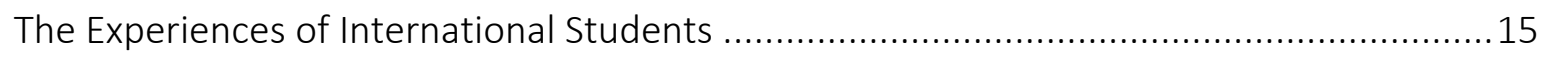

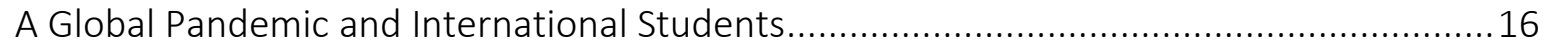

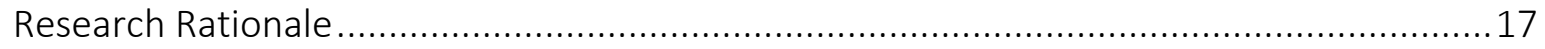

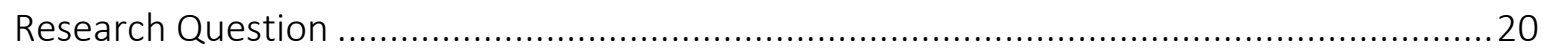

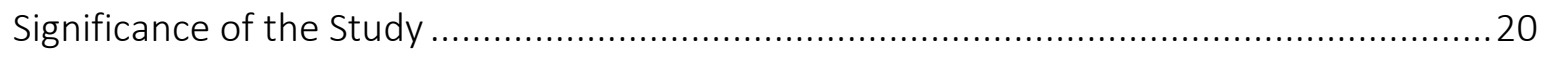

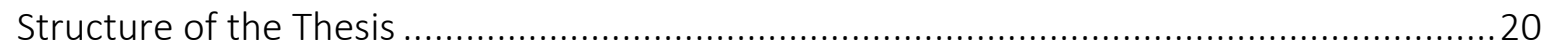

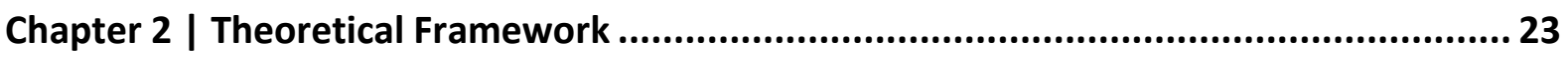

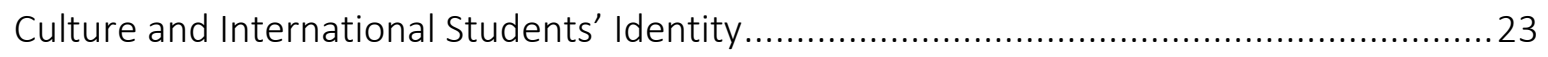

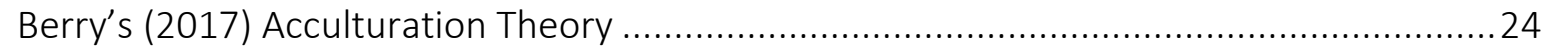

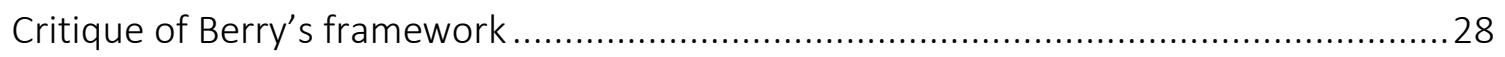

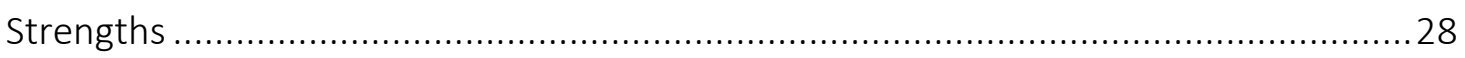

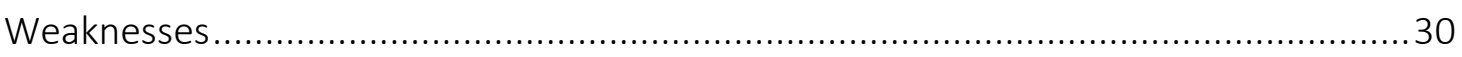

Using Berry's acculturation theory to study international students' experience ..............30

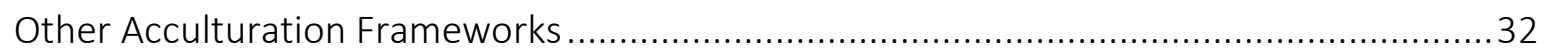

Ward et al.'s (2001; 2016) and Zhou et al.'s (2008) acculturation models ......................33

L. N. Tran's (2011) Vietnamese-specific acculturation framework................................ 38

Marginson and Colleagues' Work on International Student' Experiences ...........................41 
Marginson's (2014) theory of international students' self-formation

Marginson et al.'s (2010) concept of international student security

Critique of Marginson's (2014) idea of international students' self-formation and Marginson et al.'s (2010) conceptualisation of international student security................44

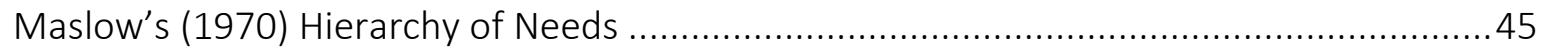

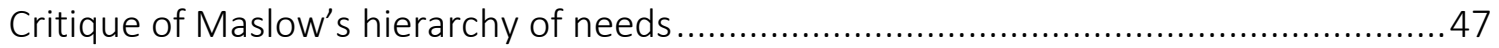

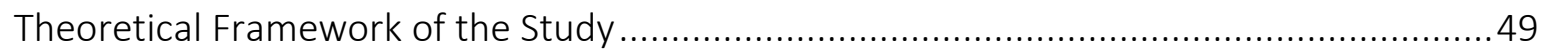

Why Were Western Theories and a Western Research Approach Used Rather Than Vietnamese Indigenous Theories and Ways of Knowing? ................................................5

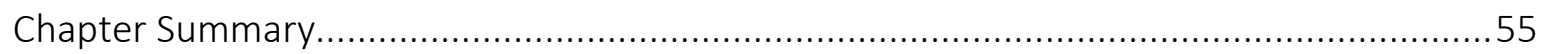

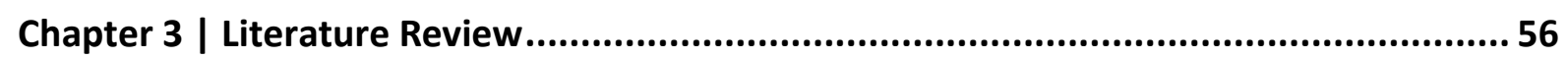

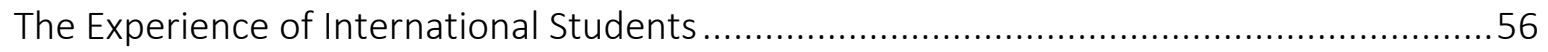

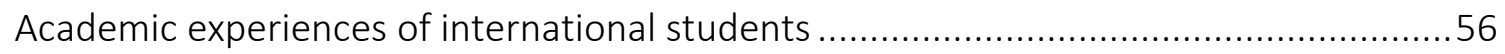

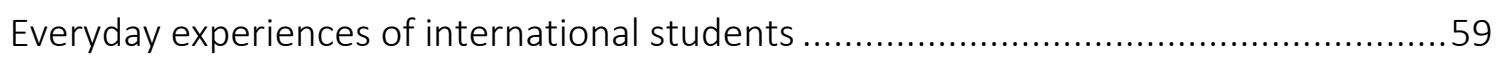

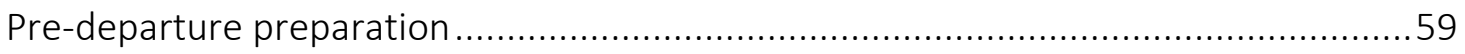

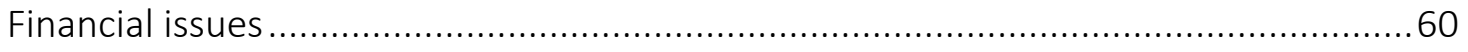

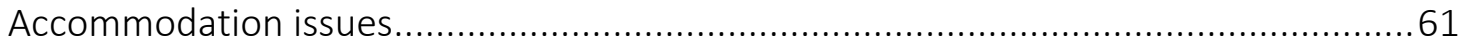

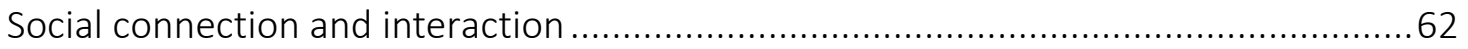

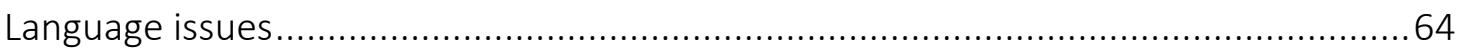

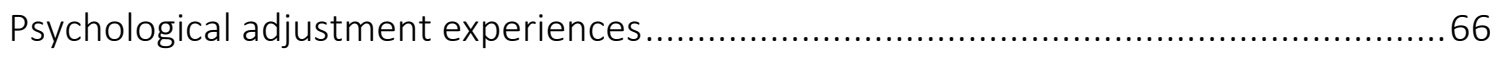

Students' strategies in dealing with challenges and help-seeking behaviours .................69

Previous Studies on Vietnamese International Students' Experiences .............................. 71

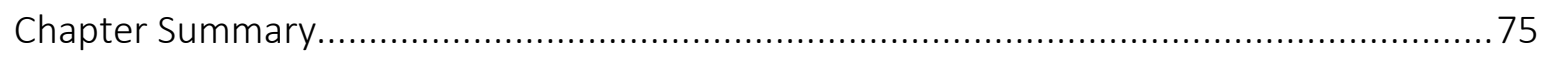

Chapter 4 | Research Methodology and Design.............................................................. 77

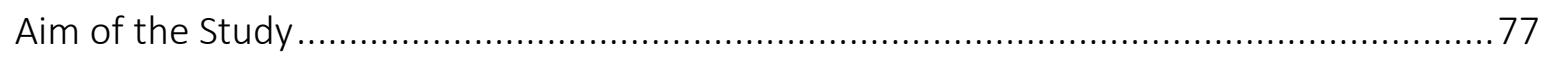

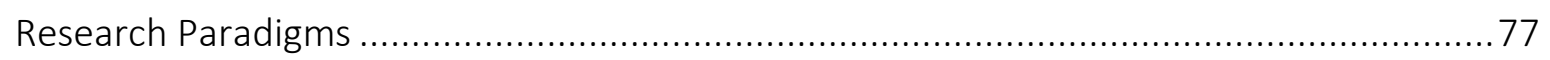

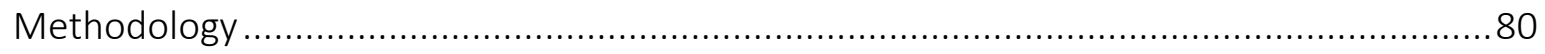

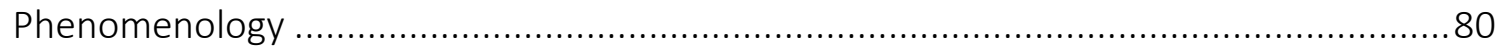

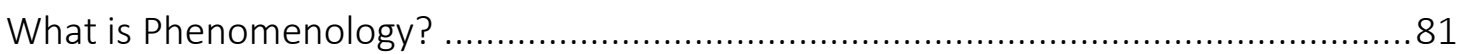

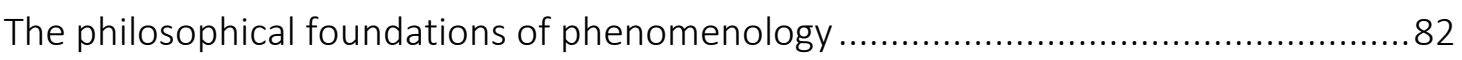

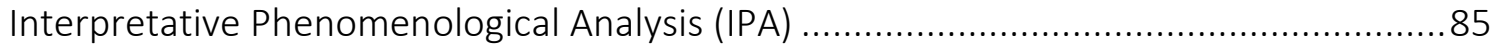

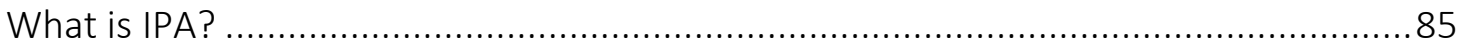

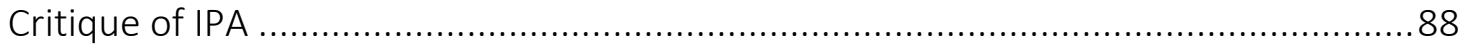


IPA or other kinds of phenomenology?

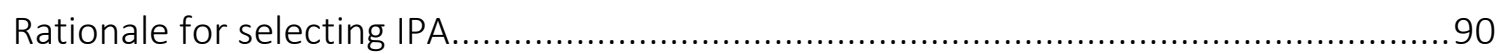

The link between the theoretical approach and IPA as a research approach .................92

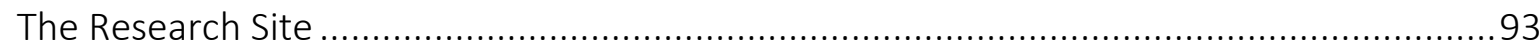

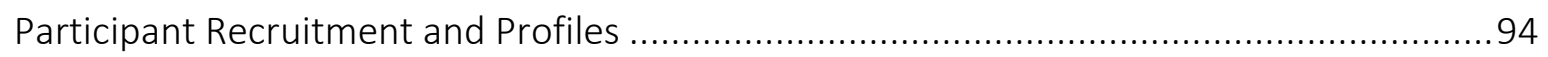

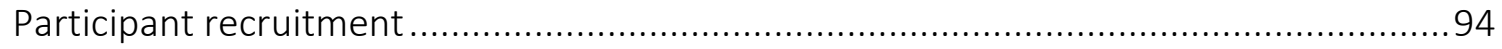

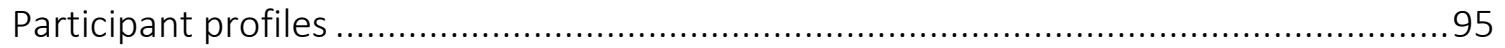

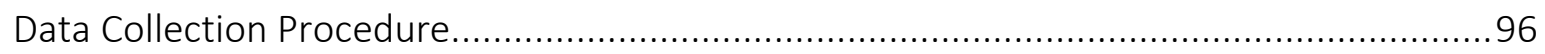

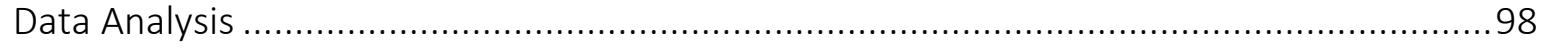

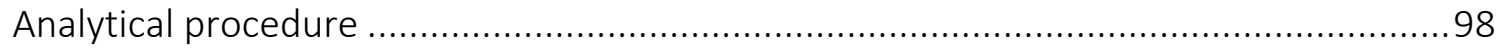

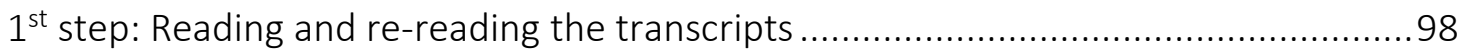

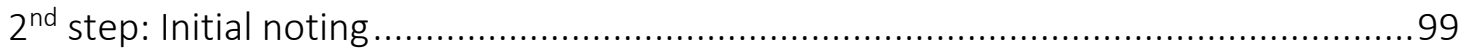

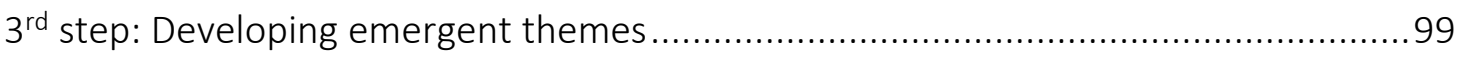

$4^{\text {th }}$ step: Searching for connections across emergent themes .................................. 100

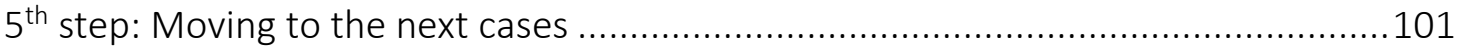

Final step: Looking for patterns across all cases..................................................... 101

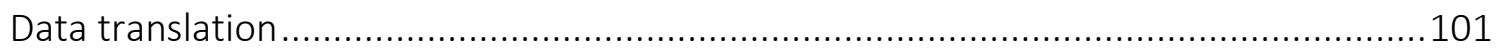

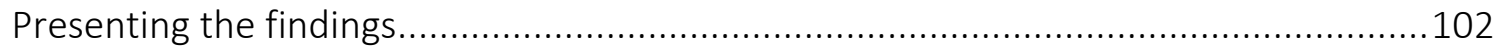

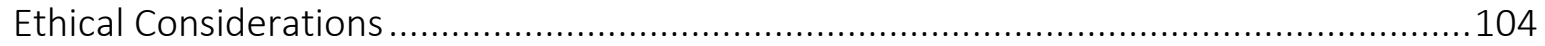

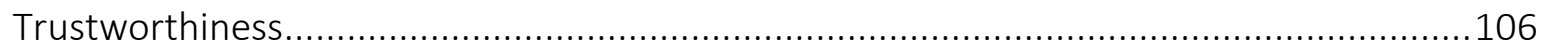

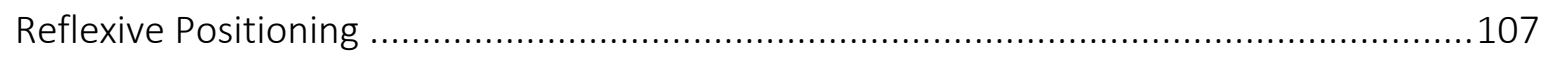

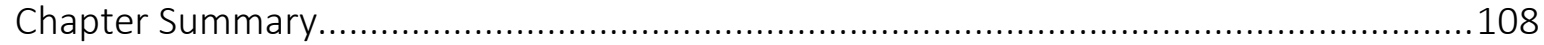

Chapter 5 | Individual Experiences: A Homogeneous but also Heterogeneous Cohort of

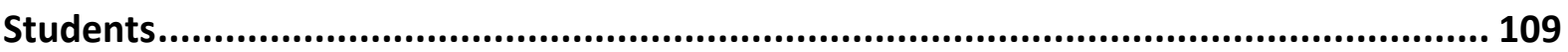

Longing for Home and the Feeling of Not Belonging …............................................ 109

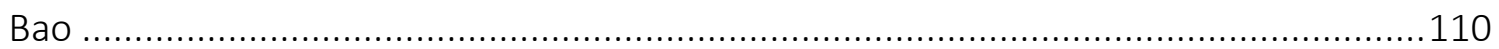

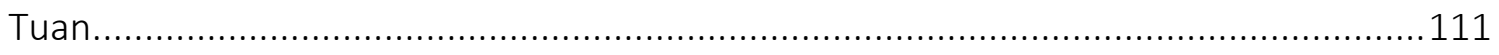

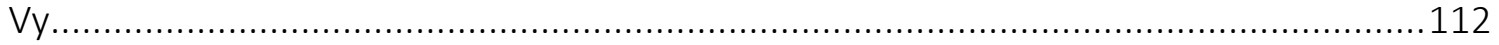

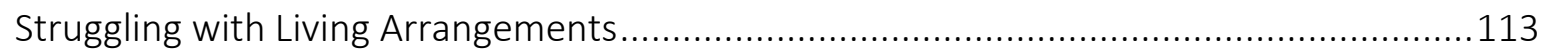

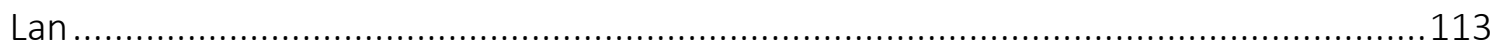

Hai

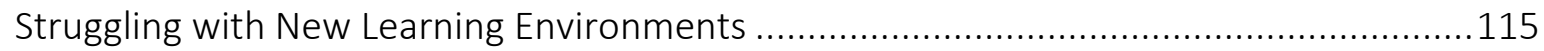


Ngoc

Tam.

Surviving and Thriving 118

Thanh 118

Ly 119

Trung 120

Chapter Summary.

121

Chapter 6 | Initial Settlement Difficulties and Learning to Live Alongside Others: Living Arrangements, Communication and Cultural Challenges 122

Living Situations of the Students 123

Challenging Living Arrangements 124

Struggling to find a place to live .124

Unfamiliar rental housing market and vulnerable tenants 128

Support from flatmates 131

Issues with cramped living spaces...... .134

Privacy and personal security 136

Issues with flatmates and landlords/landladies 137

Learning to live with others under the same roof 139

Communication and Cultural Challenges 141

Language difficulties. 141

Struggling with cultural differences 145

Chapter Summary. 148

Chapter 7 | The Quest for Knowledge and the Struggles Along the Journey: Language Difficulties and Demanding Academic Environments. 149

An Overview of the Academic Context. 149

Language Difficulties in the Academic Setting 151

Struggling with different accents 151

Struggling with writing assignments and reading materials 155

Struggling with the New Academic Environment. 157

New teaching and learning style 157

Heavy workloads .162

Issues with referencing 165 
Impacts of part-time jobs on study 167

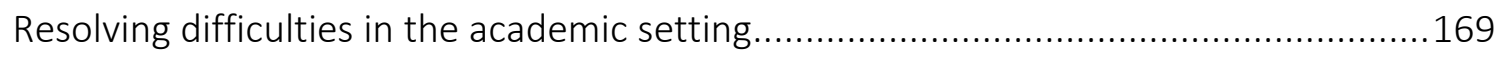

Chapter Summary.

Chapter 8 | Learning to Live on One's Own: Dislocation from Home and Friendship Networks 173

Struggling with Dislocation from Home and Familiar People ......................................174

Homesickness and yearning for connections with families and friends .......................174

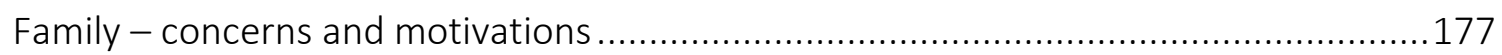

Enablers and Barriers in Forming a Meaningful Friendship.........................................179

Enablers in forming relationships with co-nationals and other Asian international students 180

Barriers in forming relationships with host-national students .................................182

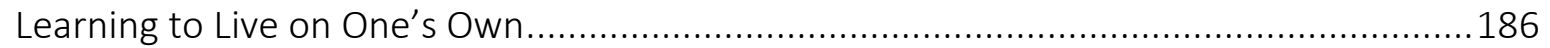

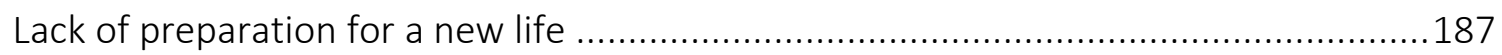

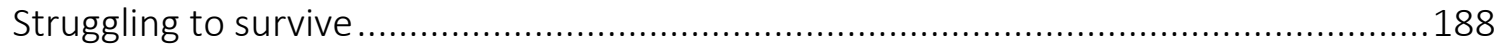

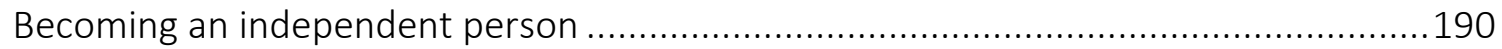

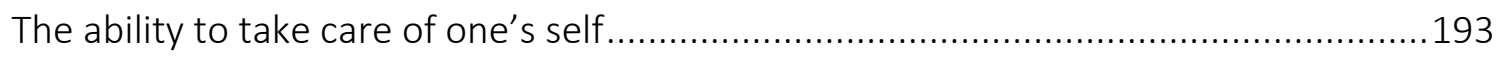

Financial independence and ability to manage budgets ............................................ 197

The Essence of Being Vietnamese Students in Aotearoa-New Zealand: Thriving Despite

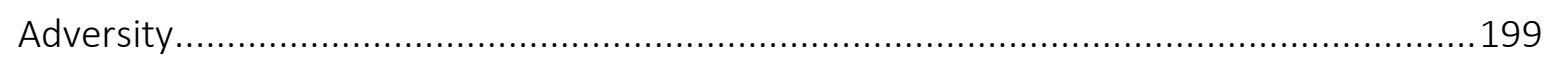

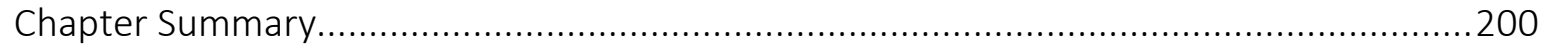

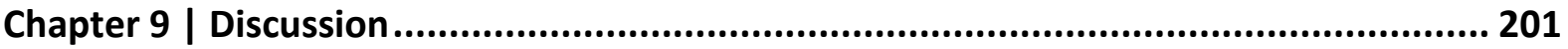

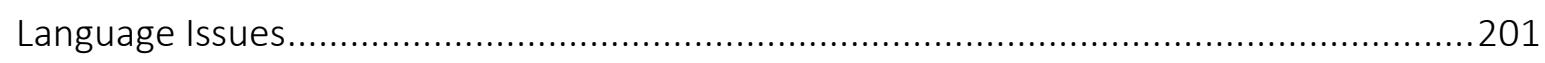

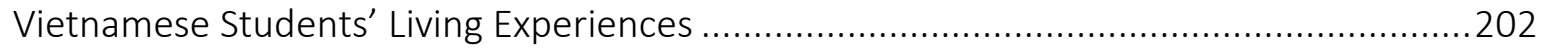

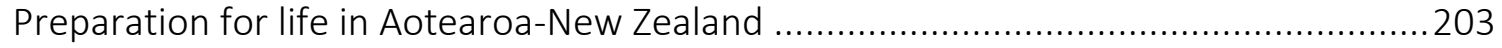

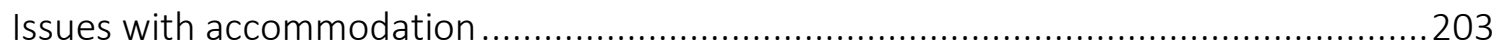

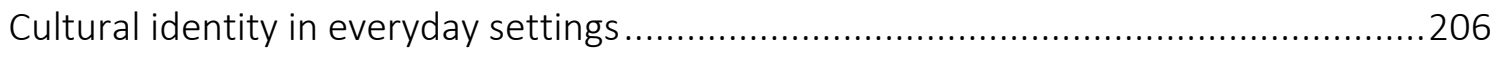

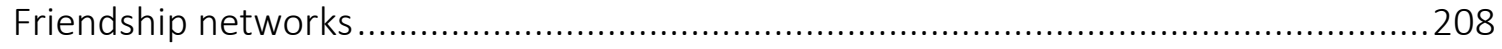

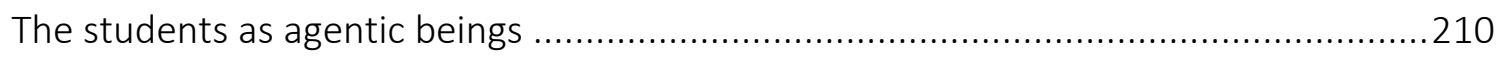

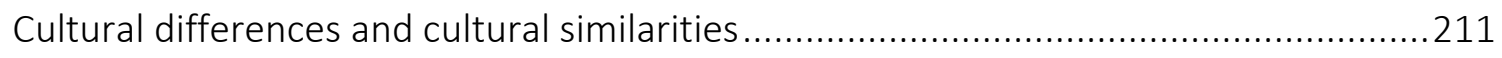

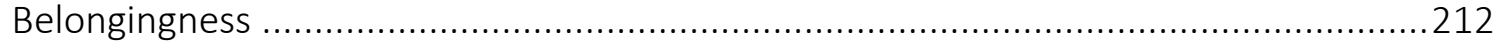

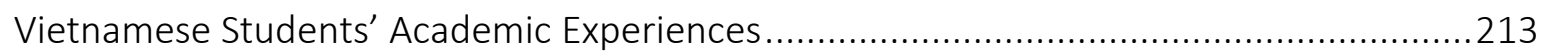


Heavy workloads

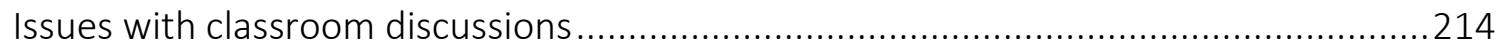

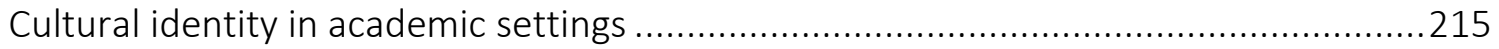

Major Contributors to the Vietnamese Students' Experiences in Aotearoa-New Zealand 215

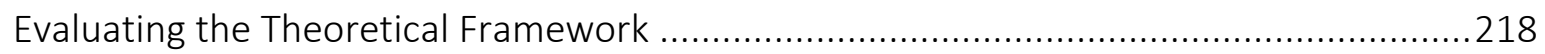

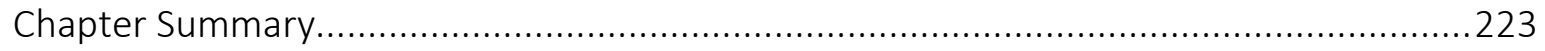

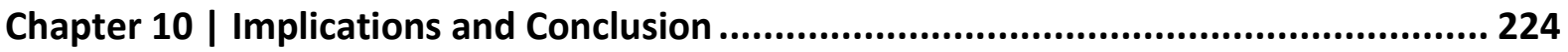

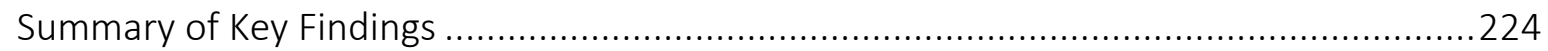

Theoretical Contributions of the Study and Reconceptualisation of the Theoretical Model 226

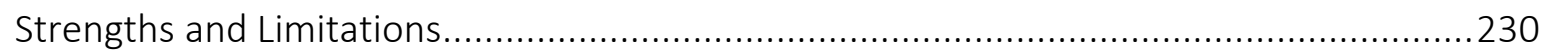

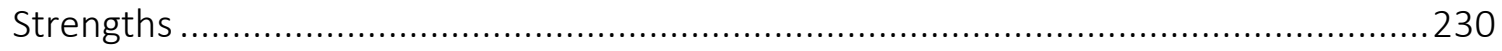

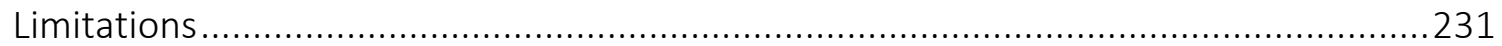

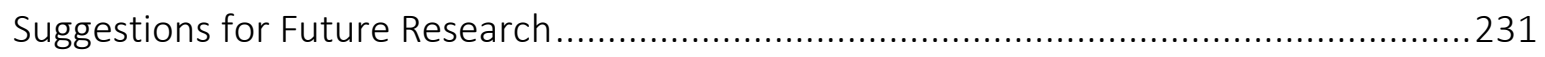

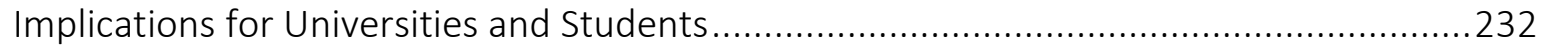

Researcher's Reflection: "The End of One Journey Marks the Beginning of Another." - Luke

Davies 233

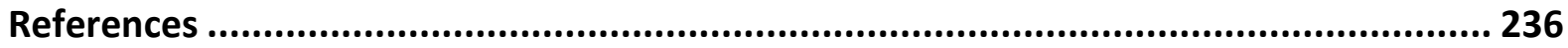

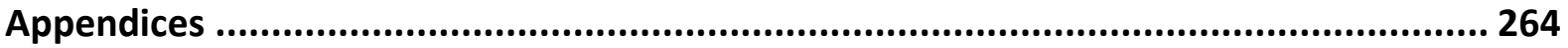

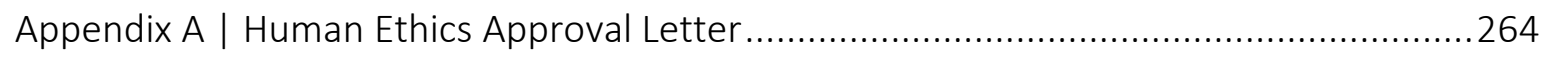

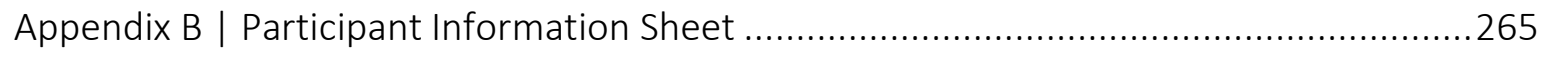

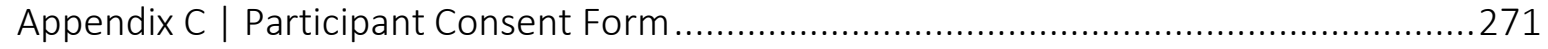

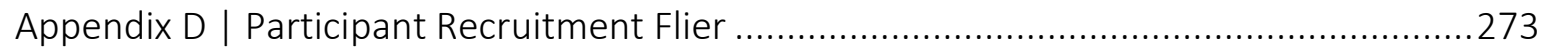

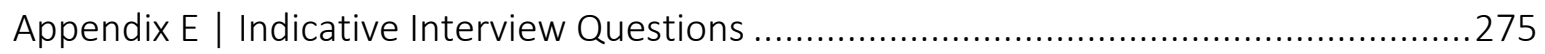

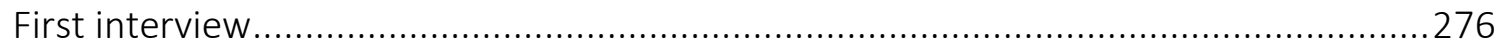

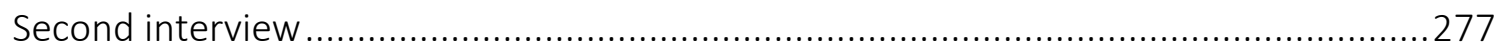

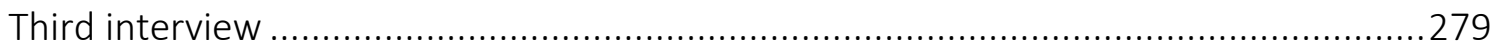

Appendix F | Summary of Studies on Vietnamese International Students' Experiences ... 280 


\section{List of Tables}

Table 4.1. Profiles of the participants...

Table 4.2. The structures of themes and sub-themes.

103

Table 6.1. Living situation changes of the students

123 


\section{List of Figures}

Figure 2.1. Acculturation framework (Berry, 2017, p. 16).

Figure 2.2. Acculturation strategies of ethnocultural groups, adapted from Berry and Sam (2016, p. 22)

Figure 2.3. The ABC model of culture shock (Ward et al., 2001, p. 271). 35

Figure 2.4. The acculturation process (Zhou et al., 2008, p. 69) 36

Figure 2.5. Acculturation process and context (Ward \& Geeraert, 2016, p. 99).....

Figure 2.6. A Vietnamese-specific psychological acculturation framework (L. N. Tran, 2011, p. 272) 40

Figure 2.7. Maslow's hierarchy of needs, adapted from Maslow (1970)..... .47

Figure 2.8. Acculturation framework for international students .51

Figure 9.1. Major contributors to the students' experiences.

Figure 10.1. Acculturation framework for short-term Vietnamese international students ... 227 


\section{Chapter 1 | Introduction}

Life is a journey that must be travelled, no matter how bad the roads and accommodations.

For many students, studying overseas is like a dream come true. But what is it like to be an international student? What do insiders talk about their first-hand experiences? Is it as rosy as what marketers of educational institutions always say? To answer these questions, in this study, I explore the experiences of Vietnamese postgraduate students in Aotearoa-New Zealand. In this chapter, I describe my own positioning and interest in the topic. I provide the background of the study and discuss the role of Aotearoa-New Zealand in international education, the mobility of Vietnamese students, Vietnamese cultural values and beliefs, and the experiences of international students. I note significant impacts that COVID-19 may have on international students, considering that this pandemic has affected several aspects of people's lives. The research rationale, research question, and significance of the study are outlined, and the chapter concludes with an overview of the thesis structure.

\section{Personal Rationale}

I came across a blog post that contributed to my aspiration to carry out this project. The post, entitled 'Study abroad: The untold story' (International student ambassadors University of Tartu, 2016), is about the experience of a Vietnamese doctoral student in Estonia. For the most part, he described his living experiences, focusing on language barriers, homesickness, and personality. Before arriving in the country, he thought he could deal with linguistic issues. However, he then felt disappointed with his English skills because he found it difficult to engage in conversations. Sometimes, he did not understand what his professors, international friends, university staff, and Estonians were saying, nor did they understand him. He was excited to discover the new living environment during the first few weeks, but felt empty, lonely, and homesick after that. He was an ambivert in that he sometimes wanted to hang out with friends, and other times he did not. But if he did not 
attend social gatherings or events, he could not make new friends, which was a dilemma. However, he found ways of tackling these problems and things got better over time. Although these issues are common to Vietnamese students who are living outside their home country, many students, including me, were unaware of them or ill-prepared to deal with them before arriving in host countries.

My interest in international students' experiences stems from my experiences as an international student in Aotearoa-New Zealand, from my friends' stories, and my hope to help other Vietnamese students to have better experiences living and studying in this country. As an English teacher, studying overseas was my dream. I wanted to experience life and cultures in English-speaking countries and use what I had learnt at university in real-life situations. I desired to gain a qualification and specialisation in the area that I have a strong interest in - international education, and hoped that with the acquired knowledge, I can contribute to my home institution. I started my journey to Aotearoa-New Zealand with positive attitudes and full of energy.

Studying at VUW has provided a lot of positive aspects. While I could study in a worldranking university with outstanding research facilities and live in a culturally diverse community, I faced many difficulties. Initially, I found it hard to understand what people were saying and to be understood. What I had learned or trained in my home country seemed to be insufficient (e.g., English skills, research methodology), causing me to feel that Aotearoa-New Zealand was a land of the unknown. I found it hard to form meaningful relationships with my flatmates, some of whom were New Zealanders and others who were of other nationalities. I experienced several misunderstandings due to cultural differences, which contributed to my limited interactions with them and my sense of disconnectedness.

My friends who studied in the USA, Australia, and the United Kingdom (the UK) for their master's degrees, have shared their experiences with me. Each had distinctive experiences adjusting to their host country. One of my colleagues, a graduate student in the USA, commented that it was challenging to make friends with local or international students from other countries. One friend who studied in Australia said that while he could establish friendship with quite a few Australian classmates, he felt that discrimination was not 
obvious, yet still around. The one in the UK shared that she felt lonely sometimes and frequently sought emotional support from her family in Vietnam.

Some of my friends who pursued a master's degree at VUW have shared their difficulties including more pressure on them due to expectations from their family and others in Vietnam and responsibilities as scholarship holders. Other observations have been to do with changes such as enhanced language proficiency, personality, interacting with people from other cultural backgrounds, changes to worldviews, and greater acceptance of other ideas and beliefs. These made me wonder whether other Vietnamese students in AotearoaNew Zealand have similar experiences, how they deal with problems, and how they feel about their experiences.

\section{Aotearoa-New Zealand's Role in International Education}

Aotearoa-New Zealand is situated in the Asia-Pacific region, and like many other nations in the Pacific region, it shares British or European settler-colonial or neo-colonial government, civic and economic systems (Marginson, Kaur, \& Sawir, 2011). The nation has a long history of involvement in international education. Although the Colombo Plan is often cited as a milestone for Aotearoa-New Zealand international education, overseas students had come to the country long before that, with students attending the Auckland-based Anglican training seminary St John's College in the late 1800s and Pacific government-assisted students in 1919 (Butcher \& McGrath, 2011). After that, students from Asia-Pacific region began to travel to Aotearoa-New Zealand for tertiary education under the Colombo Plan, which was established in 1950 by the Commonwealth foreign ministers, including Australia, Canada, Ceylon (present-day Sri Lanka), India, Pakistan, New Zealand, and the UK. The Colombo Plan served as a strategy to reduce the influence of communism on Asian countries (Meadows, 2011). Under this scheme, international education in Commonwealth countries, including Aotearoa-New Zealand, was conceptualised as an aid for student-sending countries in Asia (Butcher \& McGrath, 2011). An estimated 3500 students from South and Southeast Asian countries came to Aotearoa-New Zealand between the 1950s and 1970s (McMillan, 2016; Ziguras \& McBurnie, 2011). This scheme ended in the 1980s. 
The contemporary policy construction and provision of international education has grown to encompass offshore, onshore, and cross-national provisions and partnerships. The scope of international education includes overseas students coming to Aotearoa-New Zealand to study, students in overseas institutions using a product, services, and approaches developed in Aotearoa-New Zealand, and Aotearoa-New Zealand students studying abroad. A growing trend has been transnational education involving international branch campuses and joint programmes where students complete part of a qualification in their home countries and the other part in Aotearoa-New Zealand (Education New Zealand, 2018a). Like Australia, Aotearoa-New Zealand is attractive to international students in the region because it offers high quality education and is an English-speaking country (Ziguras \& McBurnie, 2011).

In the mid-1980s, Aotearoa-New Zealand education started to be commercialised and internationalised under the Labour government's education reform policy which encouraged educational institutions to recruit self-funded international students (Martens \& Starke, 2008; Ziguras \& McBurnie, 2011). Since then, international education has been seen as an export industry for the country. In 2019, Aotearoa-New Zealand received 61,240 international students (IIE, 2019). Although this number is small in comparison to a number of well-known host destinations (e.g., the USA, the UK, and Canada), Aotearoa-New Zealand is among the countries that have the highest international students-higher education populations ratio, which is $15.5 \%$ (IIE, 2019). The international education sector is a source of increasing revenues (ICEF Monitor, 2018a). By 2017, international students were contributing more than $\$ 5$ billion annually to the economy (Radio New Zealand, 2018). In 2017, international education was the fourth-largest export industry and the second-largest services export sector (Education New Zealand, 2018a).

In terms of the history of relations between New Zealand and Vietnam, Vietnam War (19601975), also known as the War Against America, is an important landmark. According to New Zealand History, part of the New Zealand Ministry for Culture and Heritage (n.d.), over 3000 New Zealanders came to Vietnam during the Vietnam war although their contribution to the war was modest in comparison to the world wars. New Zealand's involvement in the war was associated with their defence ties with the United States. Aotearoa-New Zealand and Vietnam established diplomatic relations in 1975 and in the ensuing years, educational 
partnerships have flourished. For example, Victoria University of Wellington (VUW) has a partnership with the Diplomatic Academy of Vietnam, and Ho Chi Minh City University of Pedagogy. Auckland University of Technology has collaborations with the Vietnam National University, and the Posts and Telecommunications Institute of Technology University. Massey University has partnered with the Vietnam National University. Joint scholarships between New Zealand and Vietnamese governments have been offered to Vietnamese students at both undergraduate and postgraduate levels (Cục hợp tác quốc tế - International cooperation department, 2020). The bilateral relationships and engagement between Vietnam and New Zealand have been strengthened in recent years. A joint statement on the strategic partnership has been approved on 22 July 2020 by Vietnamese and New Zealand Prime Ministers Nguyen Xuan Phuc and Jacinda Ardern, respectively. As such, a particular focus will be on trade, agriculture, education, and cooperation on key matters of mutual interest (New Zealand Foreign Affairs and Trade - Manatu Aorere, 2020). Education is an integral part of the bilateral ties between Vietnam and New Zealand. In one of the activities to celebrate this relationship, in late 2020, the New Zealand Embassy in Vietnam and the New Zealand Consulate General honoured six Vietnamese alumni in the New Zealand Outstanding Alumni Awards (New Zealand Education, 2020).

There have been changes in foreign policy priorities and educational philosophy in wellknown host countries, including Aotearoa-New Zealand (Butcher, McGrath, \& Stock, 2008). Historically, international education was used to serve the political and economic purposes of the provider countries (Rizvi, 2009). In the mid to late twentieth century, these countries considered international education as a form of aid to cultivate a skilled workforce for student-sending countries (Butcher et al., 2008). The reliance of student-sending countries on provider countries was strengthened during this period. More recently, international education has been viewed as a commercial enterprise, meaning that disseminating knowledge is viewed as a means for economic return. This is characterised by the recruitment of a large body of international students (L. T. Tran \& Gomes, 2017).

As non-citizens and temporary migrants, international students cannot fully access the social protection regimes that they can in their home countries (Sawir, Marginson, Nyland, Ramia, \& Rawlings-Sanaei, 2009b). In Aotearoa-New Zealand, international student security 
and quality assurance are formally regulated by The Education (Pastoral Care of International Students) Code of Practice (or The Code for short). The Code was introduced in 2001 and at that time, it was unique among English-speaking countries in terms of its regulatory framework for the provision of consumer welfare for international students (Sawir, Marginson, Nyland, Ramia, \& Rawlings-Sanaei, 2009a). Over the years, it has been revised several times. Operating at the institutional level, the Code is designed that international students in Aotearoa-New Zealand have positive experiences in relation to well-being, achievement, and rights (New Zealand Qualifications Authority, 2016). The Aotearoa-New Zealand government authorises educational institutions to be responsible for certain services and protections and oversees those institutions to make sure that their practices are aligned with those functions. Currently, two agencies - New Zealand Qualifications Authority (NZQA) and iStudent Complaints (New Zealand Qualifications Authority, 2020) - deal with complaints from international students and their authorised representatives concerning breaches of The Code.

In recent years, various strategies and policies have been established in relation to international education and international students' experience. The international Education Strategy 2018-2030, released in August 2018, set a path for the development of AotearoaNew Zealand's international education sector (Education New Zealand, 2018a) that seeks to create an environment where international education will provide economic, social, and cultural benefits for Aotearoa-New Zealand. This Strategy is underpinned by the International Student Wellbeing Strategy which was released in June 2017 and ensures that students' experience is at the heart of the international education sector (New Zealand Ministry of Education, 2017).

As part of the commercialisation of international education in the mid-1980s, Asia became the key education market for Aotearoa-New Zealand. Most international students in the country come from Asian countries, with China and India being the leading source countries whose students make up 50\% of all international students (ICEF Monitor, 2018c). Other top source countries have included Japan, Korea, USA, Thailand, Brazil, and the Philippines, in that order. A number of Asian countries have shifted from being source countries to hosting international students (Education New Zealand, 2018a). Some such as China (IIE, 2019), 
Korea (H. K. Lee \& Rhee, 2019), Malaysia, and Singapore (Doyle, 2016) have become significant providers.

\section{Mobility of Vietnamese Students}

Within the context of international education, the term 'student mobility' refers to a phenomenon in which students undertake a full degree programme outside their home countries, participate in a semester programme abroad, or study in collaborative degree programmes, such as a twinning programme (Knight, 2018). The flow of Vietnamese students overseas increased during French colonisation (1858-1954) and at that time, was mainly politically driven with the French colonial administration seeking to indoctrinate Vietnamese people into political ideologies and "understandings about civilisation" that served the political and economic needs of the French regime (C. H. Nguyen, 2018, p. 142). In that era, Vietnamese nationalists (e.g., Hồ Chí Minh, Phan Chu Trinh, and Phan Bội Châu) sought to liberate the nation through sending students to develop connections and acquire advanced knowledge in countries such as Russia, Japan, and China (C. H. Nguyen, 2018; L. T. Tran, Marginson, \& Nguyen, 2014). By the 1960s and 1970s, patterns of overseas study tended to be linked to geopolitical division in Vietnam, with Eastern Europe being the destination for tens of thousands of students from northern Vietnam while the USA and other parts of Europe being chosen by a significant but smaller number of students from southern Vietnam (London, 2010).

During the colonial period, opportunities for overseas studies were largely limited to special groups of students or the elite population (Gribble, 2011; Kelly, 2000; Meadows, 2011). However, recent trends in Vietnamese student mobility have changed and are affected by socio-political and economic changes in Vietnam and destination countries, and by transformations in international educational policies in host countries (C. H. Nguyen, 2018). Overseas study is now largely accessible to those who can afford it (M. L. T. Nguyen, 2012). Like many countries in the Asia-pacific region, Vietnam is impacted by globalisation, which requires the country to broaden its social, political, economic, and cultural relationships with other countries (London, 2010; M. L. T. Nguyen, 2012). 
Vietnam's economic reform process began in 1986 with the implementation of the 'Doi Moi' economic reforms (often known as Renovation policy) which sought to initiate the growth of the Vietnamese economic system. In recent years, Vietnam has seen a period of strong economic transformation. In response to globalisation and economic growth, education reforms were initiated. It is anticipated that these will enable the country to cultivate the skilled workforce needed for the global economy (M. L. T. Nguyen, 2012). Over a decade ago, the World Bank (2008) advised that Vietnam needed to improve its research capacity in science and technology to realise its aspiration to become a modern and industrialised country. To cope with challenges as well as to take the opportunity in the new socioeconomic period, the Vietnamese government adopted various policies and strategies to raise educational standards and internationalise higher education, for example, Education Law, the Education Law 2005, the Higher Education Renovation Agenda 2006-2020, Strategies for education development 2011-2020, and Higher Education Law. Based on the Strategy for Education Development 2011-2020, outbound mobility was considered a fundamental strategy to develop human resources and international cooperation in the field of education to enable Vietnam to catch up with countries in the region and beyond (L. T. Tran \& Marginson, 2018).

In recent years, Vietnam has become a top sending country for international students into Western education systems. The Vietnamese Ministry of Education and Training estimates that in the middle of 2020, there are around 190,000 Vietnamese students overseas and 500 collaborative degree programmes with educational institutions in over 30 countries (Vietnam Ministry of Education and Training, 2020). The Vietnamese government prioritised scholarships for students to undertake education in Russia, but self-funded students favoured Western destinations (World Education News and Reviews, 2017). In 2019, Vietnam was the sixth biggest source country for international students in the USA (IIE, 2020a), and fifth in Canada (IIE, 2020b). At the start of 2020, it is the fourth source country in Australia (Australian Education International, 2020). In Aotearoa-New Zealand, it was ranked $6^{\text {th }}$ in 2019 with 1,270 students (IIE, 2020d). Vietnam has been positioned as a strategic partner on education by Aotearoa-New Zealand government agencies (Education New Zealand, 2018b). 
Although the number of Vietnamese students in Asian countries is small compared to Western countries, the figures suggest a change in the mobility patterns in which Vietnamese students are increasingly considering studying intra-regionally, besides the Western countries that are traditional popular destinations. For example, Vietnamese students have become the second-largest international student group in South Korea (ICEF Monitor, 2019). Vietnamese enrolments saw an increase by up to 156\% between 2017 and 2019 , for a net increase of approximately 23,000 students, accounting for nearly a quarter of international students as of April 2019 (ICEF Monitor, 2019). The number of Vietnamese students in Japan varies in different sources and whether short-term abroad students are included. According to the Japan Student Services Organization (2019), in 2018, there were 72,354 Vietnamese students in the country while IIE (2020c) indicates that the number of Vietnamese students was 35,489 . In 2019 , the number of Vietnamese students in Japan increased to 42,083 (IIE, 2020c). Vietnam is the fifth-biggest student source country in Thailand (UNESCO, 2020) and was the third-largest sending market in Taiwan in 2018, numbering 12,983 students (Taiwan News, 2019). These figures illustrate the importance of Vietnam as a source country for international students and its potential for growth in "markets" that meet the needs of this student cohort.

For most Vietnamese students, the decision to study overseas is driven by socioeconomic factors, such as the lack of particular degree programmes at some Vietnamese universities, expectations of better job opportunities after graduation (in Vietnam, the host country, or another country), and students' aspirations to emigrate. Since the introduction of the 1986 Doi Moi policy (the Renovation policy), the Vietnamese government has established numerous strategies to improve the quality of the workforce and international integration, including sending students to study in other countries (C. H. Nguyen, 2018). Study abroad is explicitly encouraged for both students and academics (World Education News and Reviews, 2017). The availability of government scholarships has facilitated the outbound flow of students (H. H. Pham, 2018). Two well-known funding schemes were Project 322 launched in 2000 (Prime Minister of Socialist Republic of Vietnam, 2000) and Project 911 in 2011 (Prime Minister of Socialist Republic of Vietnam, 2010). These projects aimed to sponsor study overseas with the expectation of 20,000 doctoral graduates by 2020 . However, the 
majority of Vietnamese outbound students are not on scholarships. In 2016, 5,500 Vietnamese international students were state funded (VnExpress, 2016).

The increasing number of outbound students reflects the country's economic growth and the shortcomings of Vietnamese higher education system. Vietnamese students travel to study overseas at increasingly younger ages (ICEF Monitor, 2018b). Many Vietnamese families want their children to study in an international environment in the hope that this will contribute to their competitive economic advantage (University World News, 2019). A growing middle class in Vietnam is another factor that has been driving demand for highquality education in Vietnam and abroad (ICEF Monitor, 2018b). Domestic tertiary education appears not to satisfy many middle- and upper-class families who prefer to send their children to study abroad (ICEF Monitor, 2018b). Two possible reasons for the low level of satisfaction are graduate unemployment and highly competitive university entrance, resulting in only a small fraction of the $90 \%$ of young Vietnamese who wish to have a place in a university are able to do so (ICEF Monitor, 2018b).

The numbers of Vietnamese students overseas vary depending on sources and calculation methods, but consistently indicate an upward trend. As cited by BMI (2020), the Minister of Ministry of Education and Training - Phung Xuan Nha - estimated in late 2018 that 170,000 Vietnamese citizens were studying abroad, increasing from 130.000 in 2016. In 2018, there were 572.415 valid study abroad permit holders. According to UNESCO (2020), there are 49.386 Vietnamese tertiary-level students overseas, increasing from 55,980 in 2013, and accounting for $1.8 \%$ of total mobile students across the globe. UNESCO (2020) identified the top destination countries for Vietnamese students as Japan, the USA, Australia, South Korea, France, the UK, Finland, Germany, Canada, and Russian Federation, in that order.

\section{Teaching and Learning in the Vietnamese Higher Education}

This section examines the Vietnamese higher education in terms of teaching and learning to lay the context for the study. The Vietnamese higher education is the weakest sector in the education system and has much room for improvement (N. T. Nguyen \& Tran, 2019; L. T. Tran \& Marginson, 2018). For years, it has encountered issues such as bureaucratic obstacles and outdated curricular. The system has been described as being in a state of 
crisis, internationally isolated in terms of knowledge, and lacking high-quality universities, which fails to prepare students who can satisfy the demands of Vietnam's economy and society (Vallely \& Wilkinson, 2008). The government strategies outlined earlier in this chapter were underpinned by assumptions that the Vietnamese higher education system was not meeting the economic reform agenda. Systemic weaknesses included low teaching quality, poor qualifications of academic staff, students being ill-prepared for market needs, limited resources, and inflexible curricular. Recently, positive changes in university practices, such as the adoption of practice-based and student-centred teaching, growing emphasis on research and university-industry links have been noted (L. T. Tran, Nghia, Nguyen, \& Ngo, 2019). However, such improvements are not observed across all institutions and the Vietnamese higher education is still struggling with the identified problems (N. T. Nguyen \& Tran, 2019).

While student-centred teaching approaches have been promoted by the Vietnamese government since 2005 with the introduction of the Higher Education Reform Agenda, progress has been slow and fragmented (Nghia, Phuong, \& Huong, 2020). Traditional teaching methods, with teachers acting as transmitters of knowledge and students as passive receivers, predominate (Nghia et al., 2020), and in many classrooms, students are not given adequate opportunities for discussion or asking questions (Das, Nguyen, Nguyen, Nomikoudis, \& Dung, 2019). Teachers interact with students by asking questions and students answer based on information from textbooks (Nhat, Lien, Tinh, Hang, \& Trang, 2018). Such factors contribute to a lack of engagement in classroom discussion, which is perceived as passive in Western countries (Young, 2017).

Teachers in Vietnamese universities usually rely heavily on prescribed textbooks (Hiep, 2001; Nhat et al., 2018), which stems from a range of factors, for example, government control over textbooks (Harman, Hayden, \& Pham, 2010; D. T. Tran, Kettle, May, \& Klenowski, 2016). In a study on the role of institutional English language policies in Vietnamese university teachers' classroom practices (D. T. Tran et al., 2016), the teacher participants closely followed the prescribed textbooks and did not wish to adjust the content. They commented that keeping up with the teaching plans prescribed by their universities left them no time for other classroom activities. From an institutional point of 
view, in Vietnam, prescribed textbooks fostered standardisation of content (D. T. Tran et al., 2016). Other factors that are associated with teachers' reliance on textbooks include their heavy workload (Hiep, 2001), inadequate training and experience in adapting materials ( $\mathrm{T}$. T. M. Nguyen \& Cao, 2019).

Like many Asian countries, the relationship between students and teachers is usually formal and hierarchical in Vietnam (Harman et al., 2010; Mai \& Hall, 2017). Traditionally, the Vietnamese expected students to respect teachers, follow their instructions, and not question their knowledge (T. H. T. Pham, 2010). They viewed teachers as "having much better knowledge than students" (T. H. T. Pham, 2010, p. 31). Students avoided engaging in exchanges with teachers that might have implied criticism (C. Nguyen \& Griffin, 2010). Those who debated with teachers were viewed as discourteous and disrespectful (C. T. Nguyen, 2011). More recent evidence suggests that traditional and more interactive practices may be working in tandem in the classroom. In their study on student teachers' views of teachers and teaching in a Vietnamese institution, Mai and Hall (2017) found that the participants had unquestioning respect for the authority of their tutors. However, they were active in group work and would ask their teachers if they had questions. Mai and Hall (2017) suggested that there was some readiness from the participants to accept new teaching ideas and models, and the changes were visible but took place slowly. These cultural values may shape Vietnamese international students' relationships with their teachers and affect their participation in classroom activities.

\section{Vietnamese Cultural Values and Beliefs}

In this section, I examine some of the cultural values and beliefs that underpin Vietnamese society to set the scene for making sense of the Vietnamese students' experience. As members of cultural communities, individuals and their behaviours are shaped by societal characteristics, core values, and beliefs that predominate in their countries. In contrast to individualist societies (e.g., the USA, Ireland and Australia), in Vietnamese collectivistic society, there is a high degree of interdependence and responsibility to the group (N. T. Nguyen, 2019). Vietnamese people place emphasis on belonging and maintenance of harmony and are integrated into strong, cohesive in-groups, including their extended 
families, (Hofstede, 2011). The exercise of self-control in their everyday lives, which is associated with self-interests, personal desire and (negative) feelings and emotions, is important in maintaining harmonious relationships (J.-B. Li, Vazsonyi, \& Dou, 2018).

Vietnam has incorporated a range of values and beliefs from both ancient and modern times. Nguyen (2016) identified five major 'value layers' in Vietnamese society, including traditional values, Confucian and Taoist values, Buddhist values, Western values, and Socialist values. Amongst these layers, traditional values, Confucianism and Taoism emphasise harmony and respect for others (Q. T. N. Nguyen, 2016; L. T. Tran \& Marginson, 2014). Vietnamese cultural values and characteristics have been formed through everyday labour and throughout a long national history (Phan, 2008; L. T. Tran \& Marginson, 2014). The Vietnamese have adopted various systems of thought and combined them with local traditional beliefs to enrich their culture. They maintained elements that are suitable to Vietnamese culture and ways of living (L. T. Tran \& Marginson, 2014). The desire for harmony, which can be viewed as characteristic of the Vietnamese, guides the way they behave and make sense of their world.

Vietnamese culture privileges the ability to deal with challenges and maintain harmony (L. T. Tran \& Marginson, 2014). Vietnam is an agriculture-based country (Son, Chi, \& Kingsbury, 2019). Farmers' lives are significantly influenced by environmental conditions and climate change, and the agricultural industry involves intensive labour. Since ancient times, Vietnamese people, especially farmers, have felt a need to draw on the collective strength and solidarity of the whole community to harvest and protect crops (L. T. Tran \& Marginson, 2014). Therefore, Vietnamese farmers' ways of cultivating and living, which are flexible and communally oriented, encourages people to flexibly adapt to unfamiliar situations. Working conditions in these sectors have always drawn people together, which helps them to recognise the importance of social relations, community, and solidarity (Phan, 2008; L. T. Tran \& Marginson, 2014). The focus on communal responsibility as a social and cultural virtue shapes individuals' commitment to maintaining harmony with others. This sense of community is regarded as being emotion-oriented and is different from that of Chinese and Japanese people which is often viewed as being social-oriented (Phan, 2008). Although Vietnam is undergoing a transition to a more industrial and urban country, these values and 
beliefs still contribute to Vietnamese people's way of living and behaving (L. T. Tran \& Marginson, 2014).

The concept of harmony is one of the core ideas in Confucianism doctrines (Di Fabio \& Tsuda, 2018). Confucianism has been described as "a system of philosophical, ethical and political thought based on the teachings of Confucius" (B. D. Li, 1993, p. 305). The Confucian classics recognised the role of harmony in interpersonal relationships and social roles and suggested that strong relationships are fundamental to families and society. The Confucian concept of harmony underlines plurality and diversity. Confucianism and Taoism have at its centre harmony between individuals (Q. T. N. Nguyen, 2016). Following this principle, Vietnamese people tend to be tolerant and attempt to avoid conflict so as to maintain good relationships with others.

Vietnam can be depicted as having a Confucian heritage culture (O'Dwyer, 2017). However, it should be noted that the term 'Confucian heritage culture' implies that some cultural beliefs are common to cultures partly influenced by Confucianism, rather than suggesting that the countries have the same or shared cultures (Watkins \& Biggs, 2001). Cultural diversity among Asian countries also needs to be acknowledged (O'Dwyer, 2017).

Of the two main religions (Buddhism and Taoism/Daoism) and one philosophy (Confucianism), Taoism, which focuses on avoiding conflicts and retreating to isolation, has been suggested as the least influential in Vietnamese culture (T. D. Nguyen, 2013; Xu, 2002). Buddhism is the most influential to the Vietnamese's spiritual life. Confucianism was introduced to Vietnam under the thousand years of Chinese rule (111 BC - 938 AD) as part of the Chinese rulers' efforts to dominate the country (H. N. Nguyen, 1998). Over time, rather than being a religion, Confucianism has become part of Vietnamese culture (Vuong et al., 2018). These suggest that Vietnamese international students' behaviours and viewpoints about issues in the everyday and academic settings (e.g., their relationships with flatmates and their tendency to avoid conflict) are likely to be associated with Buddhism and/or Confucianism's values. 


\section{The Experiences of International Students}

Overseas study is not just about acquiring a degree. International students' experiences are multifaceted and related to their academic and everyday settings (Marginson, Nyland, Sawir, \& Forbes-Mewett, 2010; Ward, Bochner, \& Furnham, 2001). International students may have a mix of pleasant and less favourable experiences during their studies. Education is what makes their experiences different from those of other groups of sojourners (Ward et al., 2001).

Studying overseas may be an enriching experience for some. Lillyman and Bennett (2014) and L. T. Tran (2016) highlight aspects of personal development, including raised levels of self-confidence, independent thinking, career opportunities, and professional development. Others have found that studying abroad can develop students' intercultural competence (Maharaja, 2018; M. N. Nguyen \& Robertson, 2020).

While international students benefit from overseas educational experiences, some encounter challenges as they transition into an unfamiliar educational system (C. Smith, 2020; Z. Yan, 2020) and a new country (Dentakos, Wintre, Chavoshi, \& Wright, 2017). In leaving their homeland, they lose their usual support systems. Academic problems sometimes emerge when they encounter new academic writing conventions or teaching and learning styles (Z. Yan, 2020). Some students have difficulties with heavy workloads and oral presentations (Cowley \& Hyams-Ssekasi, 2018; J. Li, Wang, Liu, Xu, \& Cui, 2018). Reviewing the academic experiences of international students in North America, C. Smith (2020) shows that potential issues facing international students may consist of exclusion from group discussions, differences in learning cultures, and language barriers. These challenges are all potential stressors for international students (Mukminin, 2019).

Social challenges include students' ability to build meaningful relationships with locals, isolation, and housing issues (Marginson et al., 2010). The literature suggests that international students rely heavily on their co-national networks for social, psychological, and academic support (H. Li \& Pitkänen, 2018). Difficulties in forming relationships with local students are associated with language difficulties and cultural differences (H. Li \& Pitkänen, 2018; Robinson, Somerville, \& Walsworth, 2020). Some international students have not 
lived away from their extended families before studying overseas, and some lack the practical life skills needed for living independently (Forbes-Mewett \& Sawyer, 2016). Some students struggle with cooking their own meals, budgeting, sourcing food, cleaning, and organising transport and accommodation. As a result, living in a foreign country can be overwhelming. Housing issues are important to international students because they affect both wellbeing and academic achievement (Farbenblum et al., 2019; Marginson et al., 2010). Housing is prohibitively expensive in some cities (Farbenblum et al., 2019) and relationships with flatmates can sometimes be fraught (Calder et al., 2016). These have an impact on students' levels of satisfaction with their accommodation.

\section{A Global Pandemic and International Students}

This study was carried out prior to the COVID-19 (novel coronavirus disease 2019) pandemic. As this research is on international students' experiences, it is important to acknowledge that the pandemic has had a significant impact on this student cohort. The disease was first reported in Wuhan city, China in December 2019. By the time this study was in its final stages, the pandemic had made its way to hundreds of countries around the world. At the time this thesis was completed, a much-desired vaccine for the disease had not been found yet. It is still unclear how the education sector will be affected globally by the pandemic in the coming years. There have been many challenges facing international students in 2020 as the pandemic has taken hold. These have included travel restrictions, study disruptions, housing issues, unemployment, life plan changes, and mental wellbeing issues (N. C. Pham \& Shi, 2020; Sahu, 2020; Ziguras \& Tran, 2020). Loss of income from parttime employment is likely to affect students' financial situations. The pandemic has profoundly affected international student mobility (L. T. Tran, 2020). Many students cannot travel to host countries or return to their home countries because some countries are tightly controlling their borders (L. T. Tran \& Tan, 2020). Others were unable to fully experience their studies because many educational institutions around the world close and change to online teaching (Ziguras \& Tran, 2020). At the time of writing up this thesis, a number of institutions were still conducting online-only classes or adopting a hybrid of online and inperson instruction modes. The quality and flexibility of such online programmes may be a concern (L. T. Tran, 2020). 
In the USA, an announcement from the President Donald Trump released on July 6, 2020 advised that due to the pandemic, if schools were operating entirely online, international students at these establishments would not be permitted to enter the country, were required to leave America, or were advised to consider other options (e.g., transferring to another institution with face-to-face instruction) to legally remain in the country (Financial Times, 2020). In Australia, on April 3, 2020, Prime Minister Scott Morrison suggested that international students in the country consider returning to their home countries if they were unable to support themselves (Prime Minister of Australia, 2020). Besides host countries, major source countries for international students (e.g., China and India) were grappling with the health and economic effects of the pandemic (L. T. Tran \& Tan, 2020). Reflecting on the current state of global uncertainty and what is happening around the world, it may take several years for the global flows of students to get back underway. At the time I was nearing completion of this thesis, many international students were struggling with their lives and studies in this critical situation.

\section{Research Rationale}

There is a small but growing number of Vietnamese students in Aotearoa-New Zealand universities (IIE, 2020d). Given the complexity of their experiences as international students and reflecting on my personal experiences as a Vietnamese doctoral student at an AotearoaNew Zealand university, the current study sets out to explore Vietnamese students' experiences in the country.

My motivation to study Vietnamese students' experiences is driven by the dearth of studies on Vietnamese students in Aotearoa-New Zealand and the importance of better understanding the needs and priorities of this student group. While there is a growing body of research on the experiences of Vietnamese students in other Anglophone countries, especially Australia (T. A. Bui, 2021; B. T. T. Nguyen \& Pennycook, 2018; M. N. Nguyen \& Robertson, 2020), only a few studies have focused on Vietnamese students in AotearoaNew Zealand (Dao, 2005; Q. Nguyen \& Buckingham, 2019; Vu, 2013; Vu \& Doyle, 2014). Vu and Doyle (2014) explored the academic experiences of undergraduate twinning students who completed the first part of their programme in Vietnam and the second part in New 
Zealand. Vu (2013) focused on the experience of Vietnamese undergraduate students in everyday and academic settings. Others investigated students' approaches to using source materials (Q. Nguyen \& Buckingham, 2019) or their use of university libraries (Dao, 2005).

While there are a number of studies on international students' experiences in AotearoaNew Zealand, they have mostly focussed on Chinese students (Cao \& Zhang, 2012; K. Chen, 2014; Ding, 2016; Ding \& Devine, 2017, 2018; X. Wang, 2015; X. Zhang, 2018). Vietnamese students are generally viewed as a subgroup of Asian students rather than as a demographic and cultural group in their own right (Campbell, 2012; Deloitte, 2008; Franken, 2012, 2013). Although Asian students have certain commonalities in their experiences of study abroad, grouping Vietnamese students in the Asian group is problematic because it "subsumes cultural, historical, linguistic and national difference" (Matthews, 2000, p. 29) amongst students' countries of origins. The Asian region, especially Southeast Asia, comprises many countries with distinctive cultures, languages, and histories. Students' experiences of study abroad are informed by the economic and socio-cultural facets of their home countries (Berry, 2006; Wilkins, Balakrishnan, \& Huisman, 2012), and may vary considerably inter and intra-nationally (Berry, 2017; Moon, Zhang, Larke, \& James, 2020). In a study of international students in the USA (Roy, Lu, \& Loo, 2016), Chinese students found it more difficult to make social connections than their Indian counterparts, whilst Indian students reported more feelings of loneliness or homesickness than Chinese students. In a study conducted in Sydney, Australia, Brazilian and Malaysian students reported higher levels of satisfaction with housing conditions than Chinese and Nepalese students (Ryan, Dowler, Bruce, Gamage, \& Morris, 2016). Lillyman and Bennett (2014), in their review of international students' learning experiences, concluded that institutions need to consider differences in values and beliefs, traditions, and customs of students from different countries. These studies evidence the complexity of the issues facing international students, including Vietnamese students.

For many international students, the transition to postgraduate studies entails working at advanced levels of academia in a foreign language and engaging with new and unfamiliar social and academic situations (Matheson \& Sutcliffe, 2018). Jindal-Snape and Ingram (2013) used the term 'triple transitions' to describe the three difficult transitions of international postgraduate students: transition into a new educational system, a new culture, and a new 
educational level. Further, Franken (2012) recognises the problems international students face in transitioning from undergraduate study to postgraduate research, regarding it as "more complex and demanding" (p. 847). Franken suggests that insights into international students' experiences at master's level, which is the beginning of the academic journey of many doctoral students, at least in New Zealand, is important. In Aotearoa-New Zealand, studies on international students' experiences usually include students from both undergraduate and postgraduate levels (Deloitte, 2008; Kukatlapalli, Doyle, \& Bandyopadhyay, 2020; Sawir et al., 2009a; Thorup-Binger \& Charania, 2019). At postgraduate levels, researchers tend to focus on doctoral students (Ding, 2016; Ding \& Devine, 2018; Doyle, Manathunga, Prinsen, Tallon, \& Cornforth, 2017; Kidman, Manathunga, \& Cornforth, 2017). Considering the lack of research on the experience of master's students in Aotearoa-New Zealand, I decided to choose Vietnamese international students at master's level as the focus cohort for my study.

Previous research on Vietnamese students' experiences conducted in Australia (Nghia, 2015; L. Nguyen, 2018; M. N. Nguyen \& Robertson, 2020; Lien Pham, 2013; L. Pham \& Saltmarsh, 2013) and other countries (Brisset, Safdar, Lewis, \& Sabatier, 2010; T. A. Bui, 2021; Do, 2007; Miller, 2012; T. Nguyen, 2019; T. T. T. Nguyen, 2019) has identified many aspects of Vietnamese students' experiences in Western countries. These include students' self-development (L. Pham \& Saltmarsh, 2013), challenges in meeting supervisors' expectations (B. T. T. Nguyen \& Pennycook, 2018), and the role of social media in students' daily life experience (An, Lim, Lee, \& Pham, 2018). However, there are differences between Aotearoa-New Zealand as a settler society and other Western countries in terms of cultural and national identity (Rinne \& Fairweather, 2011), which may impact on international students' experiences in this country. This can be explained by drawing on acculturation theory (Berry, 2006) in which international students' experiences and motivations to study overseas are linked to social and cultural factors in host countries. Further, a number of aspects of students' experiences in the everyday setting, for example, housing experiences and negotiating relationships with flatmates, are under-researched. Coupled with the importance of housing issues in students' experiences as indicated earlier, this lack of research suggests a need to investigate how students' living arrangements may affect their lives and studies in Aotearoa-New Zealand. 


\section{Research Question}

This study aims to explore the lived experiences of Vietnamese master's students in Aotearoa-New Zealand. Specifically, I aim to investigate what difficulties the students face in their academic and everyday settings, how they deal with them, and how they enact agency in the face of challenges. The research question is:

What are Vietnamese students' lived experiences of everyday life and study in Aotearoa-New Zealand whilst they complete a master's degree?

\section{Significance of the Study}

Vietnamese students in Aotearoa-New Zealand have received insufficient attention from educational researchers. By addressing this research gap, this study makes valuable contributions to the field. It provides empirical evidence and extends knowledge about international students' experiences by investigating international students' living experiences in Aotearoa-New Zealand, focusing on Vietnamese master's students. An interpretative phenomenological approach enables me to make sense and capture the essence of the Vietnamese students' experiences, and to see what it is like to be a Vietnamese student in Aotearoa-New Zealand.

Practically, the study has implications for educational institutions in Aotearoa-New Zealand, specifically academic staff and student support services, who are concerned about effective adjustment processes of international students. An insight into Vietnamese students' experiences may help host universities provide these students with guidance and assistance which align with their problems and needs. Besides, international students, especially Vietnamese international students, can see what they may experience when studying in Aotearoa-New Zealand, which can contribute to their decision-making process, and get themselves better prepared for their lives and studies in this country.

\section{Structure of the Thesis}

This thesis is divided into ten chapters. In this chapter, I have introduced the background to the study, the research and personal rationales for the study, and its aim and significance. 
The focus of Chapter Two is on the four theories: Berry's acculturation framework, Marginson's theories of self-formation, Marginson and colleagues' theory of international student security, and Maslow's hierarchy of needs. I present a theoretical framework based on these theories.

In Chapter Three, I explain in more detail the justification for researching Vietnamese students' experiences in Aotearoa-New Zealand. I conduct a literature review on the experiences of international students, including Vietnamese students. I highlight factors that may affect students' experience (e.g., accommodation-related issues, isolation, financial burden and emotional upset) and may contribute to the uniqueness of their experiences (e.g., language barrier and discrimination). I identify the research gap that emerges from the analysis of the literature.

In Chapter Four, I present the methodology used to explore how the Vietnamese make sense of their experiences and capture the essence of these experiences. Interpretative phenomenological analysis (J. A. Smith, Flowers, \& Larkin, 2009), which is a qualitative approach, was employed. The participants were 10 Vietnamese master's students, including seven females and three males, from three programmes at a New Zealand university. I outline the stages of data collection and analysis. Ethical issues are also considered later in the chapter.

The findings are presented in four chapters (Chapters Five, Six, Seven, and Eight). Chapter Five is devoted to presenting the findings about individual experiences, highlighting their distinct characteristics and experiences in keeping with IPA's idiographic approach. I present the patterns of the students' experiences, including: (1) longing for home and feeling not belonging, (2) challenging living arrangements, (3) struggling with a new living environment, and (4) surviving and thriving.

In Chapter Six, I describe the changes in the students' living situation and how they experienced them. To aid this discussion, I explore two themes related to the students' living arrangements and cultural and linguistic challenges. The students grappled with finding a place to live and where they can feel at home. The findings on the students' housing experiences were unique contributions of my study. 
Chapter Seven deals with the students' experiences in the academic setting. I describe the academic context and then present two themes about their language difficulties and demanding academic environment. It was challenging for the students to adjust to academic practices and conventions which were different from those in their home country.

Chapter Eight consists of three themes about dislocation from home, friendship networks, and learning to live on one's own. Homesickness and difficulties in forming new relationships with other students negatively impacted the students' experiences. The chapter concludes with a discussion about the essence of the students' experience in Aotearoa-New Zealand. Their living and learning experiences were transformative in that they helped the students become independent people who were able to live in a foreign country away from their networks.

Taken together, the four Chapters Five, Six, Seven, and Eight show the students' journeys from their arrival in Aotearoa-New Zealand, finding accommodation, and studying in new and unfamiliar academic environments to their self-development in the face of difficulties.

In Chapter Nine, I discuss the findings in relation to the literature. I then highlight the major factors that contribute to the Vietnamese students' experiences with a focus on their housing, daily and academic experiences. This is followed by an evaluation of the study's theoretical framework.

The final chapter begins with a summary of key findings, highlighting the contribution of the study. I then outline its strengths and limitations, suggest directions for further research, and provide implications for universities and international students. The chapter ends with a reconceptualisation of the model and my reflection on my doctoral experiences. 


\section{Chapter 2 | Theoretical Framework}

In this chapter, I present the theoretical underpinnings of the study, including Berry's acculturation theory, Marginson's theories on international students' self-formation, Marginson and colleagues' work on human security, and Maslow's hierarchy of needs. I also critique the four theories and explain how they have informed my study.

The term 'international student' broadly refers to individuals who move from their home countries to other countries for academic purposes (Billedo, Kerkhof, Finkenauer, \& Ganzeboom, 2019; Montgomery, 2010). They may attend short courses that last a few months or longer programmes that may last for years. This term will be used throughout this study to refer to full-time students who leave their countries of origin to undertake part or all of a degree programme in another country while on a student visa.

\section{Culture and International Students' Identity}

Identity and culture as concepts are important to understand international students' experiences. Identity is "what we call ourselves and what others call us" (Marginson, 2014, p. 10). International students' identities are unique because they bring with them elements from their home countries, for example, values, beliefs, thinking, and ways of living and learning. Identity is fluid, dynamic, multifaceted and negotiable (Ting-Toomey, 2005). It is formed through social interaction and shaped by social, cultural and economic processes (Ting-Toomey, 2005). An individual may have multiple identities, which may be related to familial relations and culture (Marginson, 2014). International students' identities are socially constructed through their studies and interactions with people from diverse backgrounds (Berry, 2017; Marginson, 2014).

Culture has various definitions. Traditionally, it is conceptualised as being associated with race and nationality, which has been criticised for separating culture from characteristics of the individual and the context and thinking of culture as a static characteristic of a group of people, such as ethnicity (Rogoff, 2016). In this study, drawing on Rogoff $(2003,2016)$, I 
view culture as ways of life with many dynamic and interrelated aspects. With this perspective, human development is understood as participation in "constellations of cultural practice" (Rogoff \& Angelillo, 2002, p. 213). I pay attention to individuals' engagement with different cultural practices and communities and acknowledge that individuals change while maintaining some practices passed down from older generations.

\section{Berry's (2017) Acculturation Theory}

The increasing trend of student mobility and the important role of international students in many educational systems have required researchers to generate or utilise various theories to conceptualise their experiences (R. A. Smith \& Khawaja, 2011). One of the widely used models is Berry's (2006) acculturation framework (e.g., Krsmanovic, 2020; Ugwu \& AdamutiTrache, 2017; Q. Yu, Foroudi, \& Gupta, 2019). This theory has been influential in the development of research and theories in the field of international education.

Berry is a psychologist whose work has centred on cross-cultural psychology. He and his colleagues developed this acculturation framework (see Figure 2.1) with reference to their empirical works on intercultural contacts among ethnocultural groups, which refer to all groups within a society, including the dominant group, immigrants, asylum seekers, refugees and host community members, or indigenous people and settlers, based on the acculturation literature (Berry, 1997; Berry, Trimble, \& Olmedo, 1986).

Acculturation refers to changes that result from intercultural contact between two or more groups and their members (Berry, 2017). The contact may take place in situations such as colonisation, migration, and international study. Berry distinguishes three different types of acculturation processes based on the quality of the resulting changes, including behavioural shifts, cultural learning, and social skills acquisition. The acculturation process is unstable and can change over time. This framework draws attention to the acculturation at both group and individual levels. At a group level, acculturation usually brings about changes in one or both groups involved and may result in cultural changes (or cultural adaptations) in social structures, institutions, and cultural norms. At an individual level, it involves psychological changes (or psychological adaptations) in people's behaviours. As an example, people may learn a new language, and adopt intercultural attitudes and identities. 
Acculturation may have positive or negative impacts on an individual's experience (Berry, Kim, Minde, \& Mok, 1987). Some people may perceive their acculturation experience as stressors, but others view it as opportunities. Acculturation experience and stressors may result in acculturative stress. Contributing factors (or variables) are related to home society, host society, and individual-level factors.

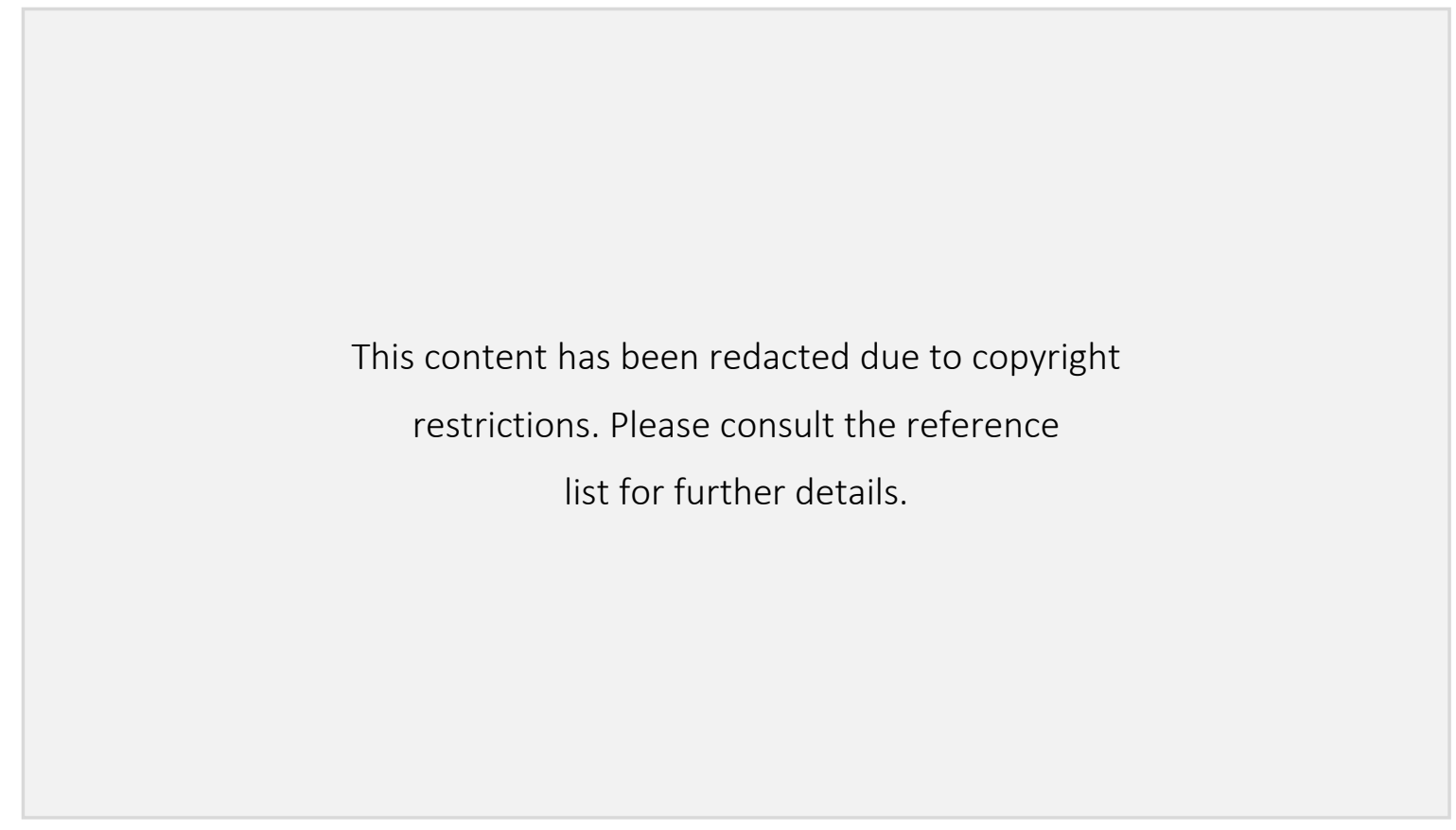

Figure 2.1. Acculturation framework (Berry, 2017, p. 16)

In Figure 2.1, original cultural groups are labelled Culture A and Culture B. A range of factors in the two societal contexts are examined in the societies of origin and settlement. In the society of origin, Berry (2006) suggested considering its cultural, political, economic, and demographic characteristics. Studying these factors may assist understanding of the cultural distance between the two cultures and the migration motivation. In the society of settlement, factors related to the society's history and general attitudes of local people towards immigration and pluralism are crucial to individuals' acculturation experience (Berry, 2006). Some societies tend to accept cultural pluralism, some wish to reduce immigration and cultural diversity, while others seek to segregate or marginalise cultural groups in their societies. Different societal attitudes tend to result in particular policies and social support for people with diverse cultures. Those factors may have a significant impact on individuals' acculturation experiences. 
Individual factors that may influence people's acculturation experiences include characteristics that exist both prior to and during the acculturation process. Individuals' characteristics before the acculturation process include age, gender, education, social status, motivation, and expectations, perceived cultural distance and personality. One potential problem for people when moving to another country is economic and social status loss, which may result in stress. Berry (2006) suggests that the acculturation process is generally smoother for younger individuals and difficult for adolescents. Females may encounter more acculturation difficulties than males. Higher education is associated with lower levels of acculturative stress and better adaptation to unfamiliar cultural settings.

Motivation and expectations of migration are usually studied using the push/pull model proposed by Lee (1966). Push factors are located within students' country of origin, exerting pressure for them to move overseas for higher education whilst pull factors emanate from the destination country, attracting students to come for education (Altbach, 1998; Mazzarol \& Soutar, 2002; Wilkins et al., 2012). There are relationships between motivations and adaptation (Berry, 2006). For example, people with high push motivation might encounter more difficulties in psychological adaptation. Greater cultural distance - the perceived cultural differences between the societies of origin and settlement - are generally associated with poorer adaptation (Berry, 2006). Regarding the relationships between personality traits and adaptation, there are few consistent findings, but Berry (2006) suggests that if one's personal characteristics 'fit' well with the new society, they may facilitate the process of adaptation. Factors that emerge during acculturation consist of the length of acculturation and social support (Berry, 2006). How long people have been undergoing acculturation may affect the kind and level of difficulties they encounter. Supportive relationships with both home and host cultures positively affect individuals' psychological adaptation.

At the individual level, Berry (2017) takes into account two aspects of the acculturation process: psychological acculturation and adaptation. Psychological acculturation involves all groups in contact and includes behavioural changes, acculturative stress, and acculturation strategies. Behavioural changes may relate to development in language knowledge and 
skills, cultural identities, and personalities. Acculturative stress results from challenges facing individuals in the acculturation process and is associated with anxiety and depression.

Acculturation strategies (see Figure 2.2) are a key theme in the acculturation model and relate to how people choose to engage with the acculturation process, including integration, marginalisation, assimilation, and separation (Berry, 2017). Berry conceptualises individuals' acculturation strategies in relation to two issues: maintenance of heritage culture and relationships or interactions among groups. With integration, individuals both maintain their cultural identity and seek interaction with the host culture. With marginalisation, they neither maintain their cultural identity nor have relations with others in the host culture. Assimilation is adopted when individuals abandon their cultural identity and wish to absorb elements of the host culture. Finally, separation involves individuals' maintenance of their heritage culture and avoidance of interaction with the host culture. In some contexts (e.g., indigenous people in settler nations), people from minority groups may have limited freedom in adopting the strategies they want (Berry, 2017), and forced assimilation and marginalisation may happen (Dudgeon et al., 2016). People from one cultural group may employ different strategies in different situations. Strategies used may be associated with various levels of adaptation, with integration probably being the most effective strategy, in comparison to assimilation, separation, and marginalisation (Sam \& Berry, 2010).

Issue 1:

Maintenance of heritage culture and identity

Issue 2:

Relationships sought among groups

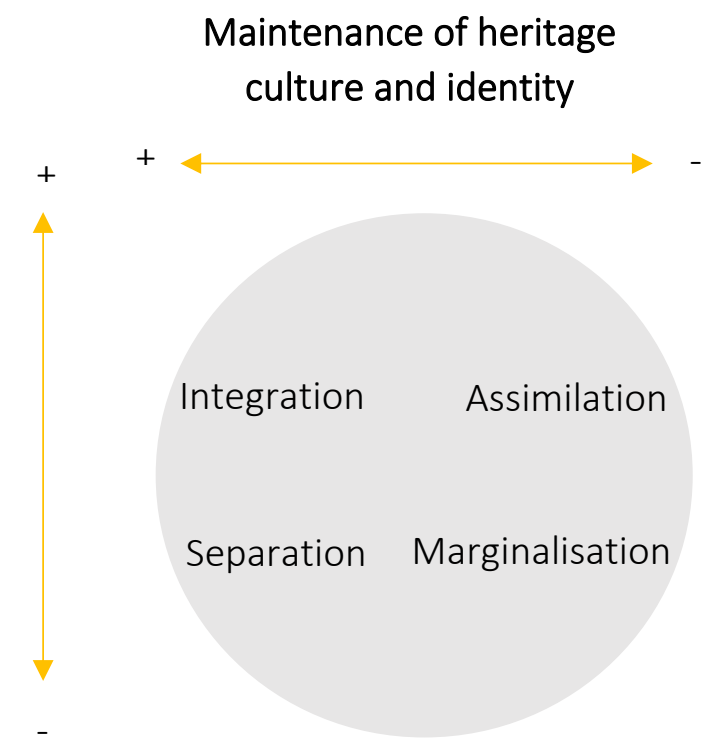

Figure 2.2. Acculturation strategies of ethnocultural groups, adapted from Berry and Sam (2016, p. 22) 
Adaptation is defined as "the relatively stable changes that take place in an individual or group in response to external demands" (Berry, 2006, p. 52), and includes three aspects sociocultural, psychological, and intercultural adaptation (Berry, 2017). Different people may have different levels of success in adapting to new environments (Berry, 2017). Sociocultural adaptation is associated with individuals' ability to perform well in unfamiliar intercultural environments, such as the international educational context. Psychological adaptation involves their mental and physical health while sociocultural adaptation is related to their ability to effectively deal with issues in an unfamiliar cultural setting. Intercultural adaptation has at its centre individuals' ability to form workable relationships with people from different cultural backgrounds (Berry, 2017).

As a reiteration, Berry's framework was originally designed to be used with immigrants. Although both immigrants and international students' experiences may be affected by factors in host and home countries, there are marked differences between the two groups. For example, while immigrants seek to permanently reside in host countries, international students may choose to stay in host countries, return to their home countries, or migrate to another country upon completion of their degrees. Due to the length of their academic programme, some students may stay in host countries for a shorter period than others. Such differences raise a question about how they may affect international students' acculturation experiences (e.g., their acculturation strategies).

\section{Critique of Berry's framework}

Although researchers have widely applied Berry's acculturation framework and recognised its value (Ward \& Kus, 2012; Yoon, Langrehr, \& Ong, 2011), this framework is not without weaknesses. This section aims to shed light on some strengths and weaknesses of Berry's acculturation framework.

\section{Strengths}

Berry's acculturation framework is complex and includes a range of variables needed for a study on acculturation (Ward, 2008; Yoon et al., 2011). It demonstrates the vital role of situational factors in the acculturation process and outcomes. In the international education context, the critical role of such factors (e.g., teaching and learning practice in host countries 
and students' living arrangements), which will be discussed in the next chapter, becomes apparent. Furthermore, this model highlights both the process of adjustment to multiple life stressors over time and how pre-arrival and post-arrival factors might impact on the adjustment process. The four acculturation strategies are widely used in the literature on international students' acculturative experiences (Avolonto, 2020; Krsmanovic, 2020; Xing, Popp, \& Price, 2020).

Whilst Berry's model was initially developed to investigate immigrants and refugees' experience, it has been adapted to be used with various groups of population such as businesspeople and tourists (Ward et al., 2001). Berry (1997) points to this possibility by using the metaphors of 'skeleton' and 'flesh'. He views his framework as an open structure that has the potential to incorporate new elements. This allows researchers to adapt the framework in their research and obtain a better understanding of the experience of their target participants. As shown in a later section, researchers have modified the framework by adding new elements or clarifying relationships among existing elements (L. N. Tran, 2011; Ward \& Geeraert, 2016; Zhou, Jindal-Snape, Topping, \& Todman, 2008).

The framework makes clear and conceptualises a number of factors (Ward, 1997). For instance, Berry distinguished between two terms - assimilation and acculturation - that are sometimes used interchangeably by others (Sam \& Berry, 2010). Assimilation is one of the four strategies that individuals may adopt in intercultural contact, and acculturation is the process which at the group level involves the bi-directional cultural contact between different cultural groups (Berry, 2009, 2017). Although Berry views this as a bi-directional process, this may not always be the reality, for example, the situations of indigenous people in settler nations (Dudgeon et al., 2016).

Another significant conceptualisation proposed by Berry (1997) is the stress and coping framework, which suggests that sojourners may encounter stressful life changes and need to develop strategies to cope with them (Berry, 2006). Berry's framework helps explain the factors that affect acculturative stress and adaptation. It has integrated three theories - the culture learning approach, the psychological model of stress, and the mental health disease perspective, which clarifies the acculturation process (Ward, 1997). 
Finally, the distinction between the two levels of acculturation (i.e. individual and group) is critical because it allows researchers to examine the different acculturation forms and their relationship at the individual, group, and societal levels (Adler \& Gielen, 2003). In terms of international education, such relationships are useful because they demonstrate how factors at different levels may affect international students' experiences. The various types of acculturation attitudes and behavioural strategies of individuals from a minority group and a host society can also be categorised and explained (Adler \& Gielen, 2003).

\section{Weaknesses}

Whilst the framework's advantages are recognised, its disadvantages need to be acknowledged. The framework pays insufficient attention to the critical role of individuals' agency in their acculturation experiences. In the context of international education, a regrettable trend in the use of Berry's acculturation framework has been that researchers failed to acknowledge students' agency and potentially portrayed them as deficit agents whose experiences are largely shaped by factors from the outside world, such as social support and language (Ra \& Trusty, 2017; Szabó, Papp, \& Luu, 2020).

Ward and Geeraert (2016) suggest that Berry's four acculturation strategies are not always found in empirical research. Findings from a number of studies using latent profile analysis are inconsistent with the four strategies proposed by Berry. Separation and integration frequently occur, assimilation is regularly noted, and marginalisation is rarely reported.

\section{Using Berry's acculturation theory to study international students' experience}

Early acculturation researchers were mainly interested in matters related to European colonisation and indigenous peoples (Berry, 2017). This was followed by increasing interest in examining issues related to immigrant and ethnocultural groups. More recently, impacted by globalisation and trading and political relations, much of the work has involved studying indigenous national populations, immigrants, refugees, and sojourners.

Within the literature on acculturation, international students are generally considered as sojourners (Berry, 2017; Ward et al., 2001). A sojourn refers to "a temporary stay in a new 
place" (Ward et al., 2001, p. 142) and this occurs voluntarily. Sojourners may include international businesspeople, diplomats, international students, and missionaries (Ward et al., 2001). International students are a commonly researched group because of their accessibility as research participants (Zhou et al., 2008).

The experience of international students, like that of other cultural travellers, may be affected by many factors related to home and host countries, which are often studied in relation to push-pull factors proposed by E. S. Lee (1966). Although the push-pull model was originally used to explain reasons for people's migration, it has been frequently used over decades by educational researchers to explain international students' motivations and decisions to study overseas, and their choices of destination countries and institutions (Mazzarol \& Soutar, 2002). Jamaludin, Sam, and Sandal (2018), in a study on international students in Norway, found that destination motivation (pull factors) is one of the most important variables influencing students' decision to revisit and to recommend the destination. K. Yan (2017) notes that unmet expectations may contribute to stress that students encounter. High motivation may lead to high expectations of students' academic performance, which may increase academic stress. To maintain their desired academic results, students may need to work harder, which possibly makes them more stressed.

Many individual and situational characteristics may impact on students' experience in host countries. Systematic reviews on the adjustment of international students have suggested many factors, for example, acculturative stress, social support, country of origin, length of residence, and English language proficiency (Brunsting, Zachry, \& Takeuchi, 2018; Mesidor \& Sly, 2016; R. A. Smith \& Khawaja, 2011). Ra and Trusty (2017), exploring the experience of East Asian students in the USA, found that social support and coping strategies were partial moderators on the relationship between acculturation and acculturative stress. In their study on international students in Korea, Kim et al. (2019) found that students with depressive symptoms might enhance their quality of life if they adopted the integration strategy. Ladum and Burkholder (2019), examining the experience of international students in the Northern Part of Cyprus, noted that low social support and language proficiency might be related to students' acculturative stress and emotional responses. 
In Berry's acculturation framework, adaptation may be divided into psychological and sociocultural adaptations, which link individuals to others in the host community and indicate their ability to effectively deal with everyday intercultural activities, for example, social relations and academic success (Berry, 2017). International students may have three social networks - co-national, host-national, and multinational networks (Bochner, McLeod, \& Lin, 1977; Ward et al., 2001), in which each has different functions. Social support can affect students' psychological and academic adjustment. Co-national friends may provide useful information for daily life, and support with social, psychological, and academic issues, such as planning time schedules for courses, discussing assignments, sharing research experience, and proofreading written work (H. Li \& Pitkänen, 2018). Chinese students in a Thai study reported that same-culture relationships help them deal with homesickness (Lin \& Kingminghae, 2014). Quality contact with the host society may facilitate students' crosscultural learning and personal development (Y. Yu \& Moskal, 2019). Friendship with local students might enrich their personal lives $(\mathrm{H}$. Li, 2017). Frequent communicating and interacting with other international students may help students improve their language ability and cultural knowledge (H. Li \& Pitkänen, 2018).

There are fundamental similarities and differences between the experiences of international students and other groups of sojourners and cultural travellers. International students and businesspeople are generally well educated and highly motivated (Ward et al., 2001). However, many students are from less developed countries and undertake higher education in developed countries while businesspeople tend to take the opposite movement (Ward et al., 2001). Berry (2017) noted that international students may leave their education countries and return home or go to another country, whereas immigrants are permanent residents. This may lead international students to be less involved in the local community and hesitant to form meaningful relationships. However, this is not always the case as students' experience is complicated.

\section{Other Acculturation Frameworks}

A number of acculturation models have been developed based on Berry's acculturation framework. In this section, I outline the evolution of research on acculturation and introduce 
four acculturation frameworks, which contribute to my theorisation of the experience of Vietnamese international students in New Zealand.

\section{Ward et al.'s (2001; 2016) and Zhou et al.'s (2008) acculturation models}

An unpleasant experience that cultural travellers have in a host country is generally termed 'culture shock'. Coupled with 'reverse culture shock', this term has been widely used in the popular media (Furnham, 2019) and employed in studies on international students' experiences (Belford, 2017; Furnham, 2019; Presbitero, 2016). The concept of culture shock is used by many international students because it resonates with them and provides some reassurance that what they experience has been encountered by others (Belford, 2017; Heng, 2017). Some of the major international student-run websites use these concepts as in UKCISA (UK Council for International Student Affairs). In his acculturation framework, Berry (2016) has used the term 'acculturative stress' in place of 'culture shock', acknowledging that the two terms are similar. However, within the stress and coping framework, the term 'stress' has a more positive connotation than that of 'shock' because in coping with stressful experiences, individuals adapt to challenging situations (Berry, 2006). Besides, the term 'acculturative' indicates the source of negative experiences, which is the intercultural contacts of ethnocultural groups (Berry, 2006).

First used in 1960 by an anthropologist - Oberg (Pusch, 2004), the concept 'culture shock' was initially used to explore people's intercultural experience. In the past, this term was used to investigate immigrants' experiences relating to mental health (Furnham, 2019). After the 1950s, research on overseas students focused on social and psychological problems. Historically, affected by a clinical perspective, research on international students tended to utilise medical models of sojourner adjustment, focusing on negative aspects of their experiences (Ward et al., 2001). Medical models of culture shock have been criticised for viewing sojourners as passive victims who need outside help (Ward et al., 2001).

In the 1980s, researchers began to adopt a different perspective which viewed sojourning as a learning experience, or the acquisition of skills relevant to the new culture. Based on this perspective, the culture learning model was constructed. Culture shock tended to be studied from the viewpoints of social psychology and education, which subscribe to three 
theoretical approaches established earlier - social skills/culture learning model, stress and coping model, and social identification theories.

Originating from social psychology, the stress and coping model is related to the impact of life events. Berry (1997) was one of the first to develop a stress and coping model to explain the factors affecting acculturative stress and adaptation. This model deals with the affective and emotional effect of cultural contact which leads to psychological adaptation (Ward et al., 2001). Culture learning theory comes from social and experimental psychology, focuses on behavioural aspects of intercultural contact, and regards social interaction as a skilled performance (Argyle, 1969). This model takes into account the behavioural changes that a person makes to adjust to a new environment, and this process is related to sociocultural adaptation (Ward et al., 2001). Social identification theories, having their origins in ethnic, cross-cultural and social psychology, consider the cognitive elements of the adaptation process. This change in the cognition domain results from the psychological and sociocultural adaptation (Ward et al., 2001). In brief, these three theories approach issues of sojourners' responses to difficulties encountered in unfamiliar cultures in relation to three changes - affective, behavioural and cognitive $(A B C)$ respectively.

Incorporating these theories and expanding on Berry's (1997; 2006) acculturation framework, Ward et al. (2001) developed a model to study intercultural contact and change, which they call the ABC model of culture shock (see Figure 2.3). 


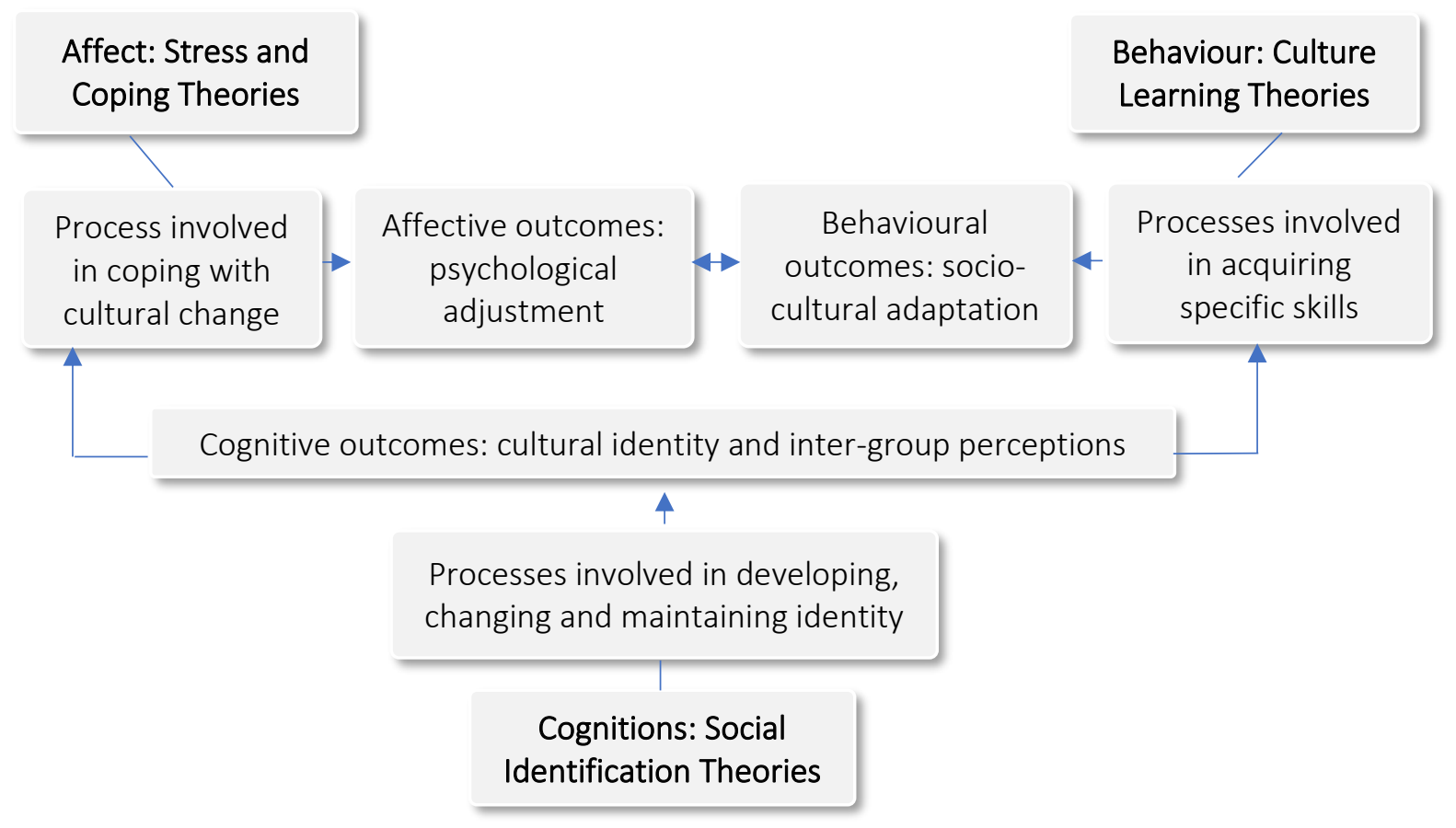

Figure 2.3. The ABC model of culture shock (Ward et al., 2001, p. 271)

There are strengths and weaknesses in the ABC model. Zhou et al. (2008) in reviewing this model in relation to its relevance to student sojourners made four points. First, the model is more comprehensive than the earlier ones in that it distinguishes three elements of human interactions by incorporating the three theoretical perspectives to study culture shock. Second, it views acculturation as a dynamic process that occurs over time, not at one time. Third, it highlights sojourners' active responses to negative experiences. Fourth, the model includes the characteristics of the situation as well as those of the sojourners. Finally, it studies culture shock from educational perspectives rather than clinical views, which may give implications for the use of the cost-effective intervention.

Despite such advantages, Zhou et al. (2008) criticise the model as complex and note that researchers might find it difficult to observe the relative effects of each factor. Further, there is a lack of integration between the three theories (i.e., stress and coping theory, culture learning theory and social identification theory) and research on the psychology of different groups of cross-cultural travellers. On this basis, the authors suggest that future researchers improve the framework by combining theories into a general model. 
Zhou et al. (2008) adapted Ward et al.'s (2001) ABC model by linking the stress and coping strategies with the acquisition of skills relevant to host culture, and distinguishing psychological, sociocultural, and cognitive outcomes (see Figure 2.4). They emphasised the interaction between the 'cognitive' to the 'psychological' and 'sociocultural' and added arrows to the model.

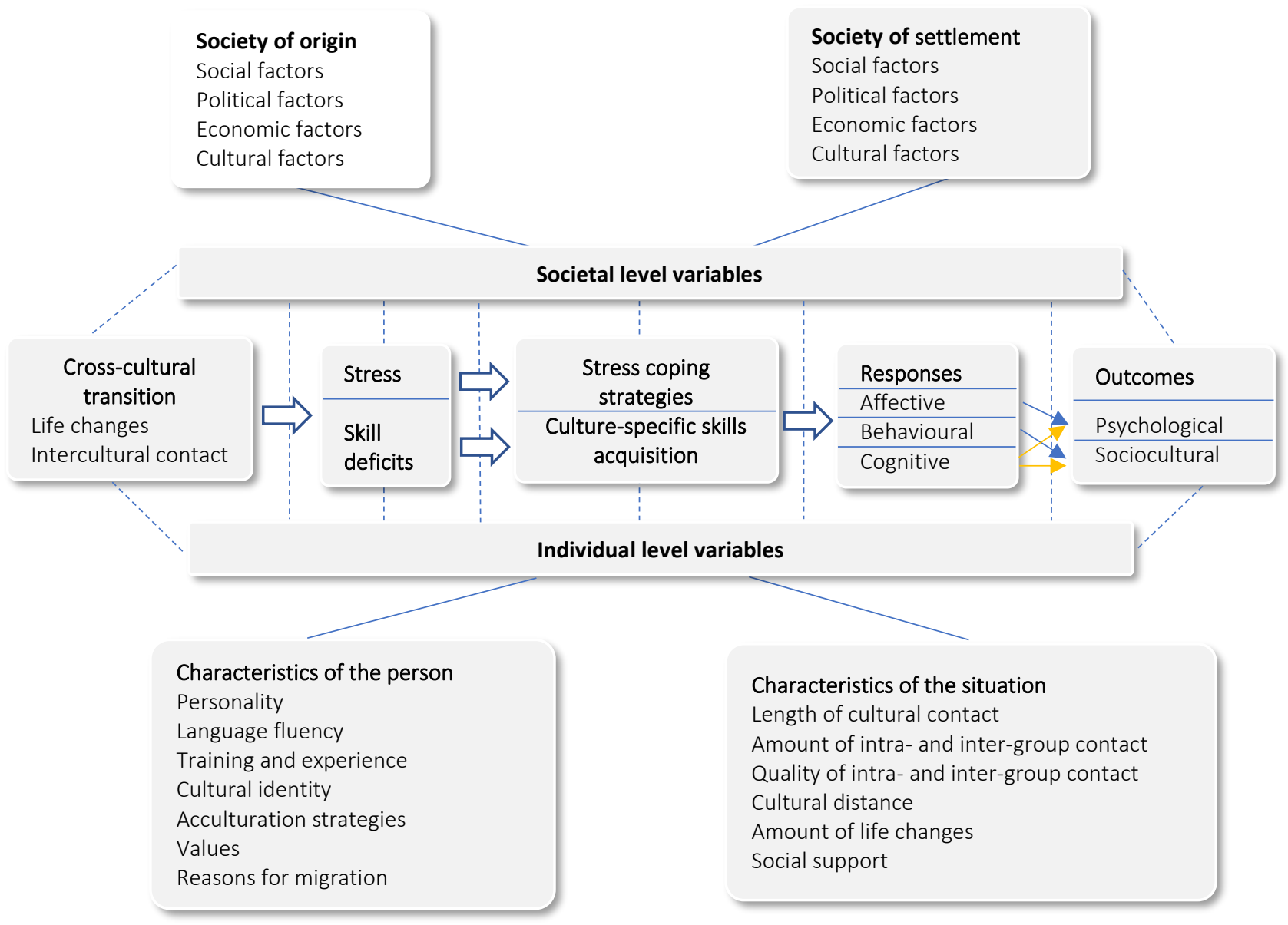

Figure 2.4. The acculturation process (Zhou et al., 2008, p. 69)

Although highlighting the efficiency and comprehensiveness of their acculturation model, Zhou et al. (2008) indicated that it has some weaknesses. First, there is a lack of clarity in the relationship between psychological and sociocultural adaptation. Second, the cognitive aspects of acculturation appear not to fit with the acculturation process. Finally, the relationship between students' pedagogical adaptation and their psychological and sociocultural adaptation needs clarifying.

Drawing on the literature on immigrants' acculturative experiences, Ward and Geeraert (2016) propose a process model of acculturation (see Figure 2.5) and emphasise the 
interdependence and dynamic relationships among the elements. In this model, they put intercultural contact at the heart, consider it as the starting point of the acculturation process, and emphasise the critical role of individuals' orientation to the cultures of their home and host countries. Ward and Geeraert (2016) highlight the ecological context in which intercultural contact happens, including societal, institutional and organisational, and familial contexts. These contexts are considered in both home and host cultures and collectively affect individuals' acculturative experiences. Familial factors may include parental expectations and family functioning and may have a significant impact on young people's experiences. Familial context in host countries is particularly related to individuals who are accompanied by their families. Institutional and organisational contexts are related to educational institutions and work environments. Societal context is associated with such issues as societal attitudes towards cultural differences and host countries' policies about multiculturalism. Ward and Geeraert (2016) position the acculturation process within a wider ecological context of global culture and take into account the trend of globalisation and transnationalism. Such global trends may significantly impact on how the acculturation process may happen. For example, remote acculturation may take place through media.

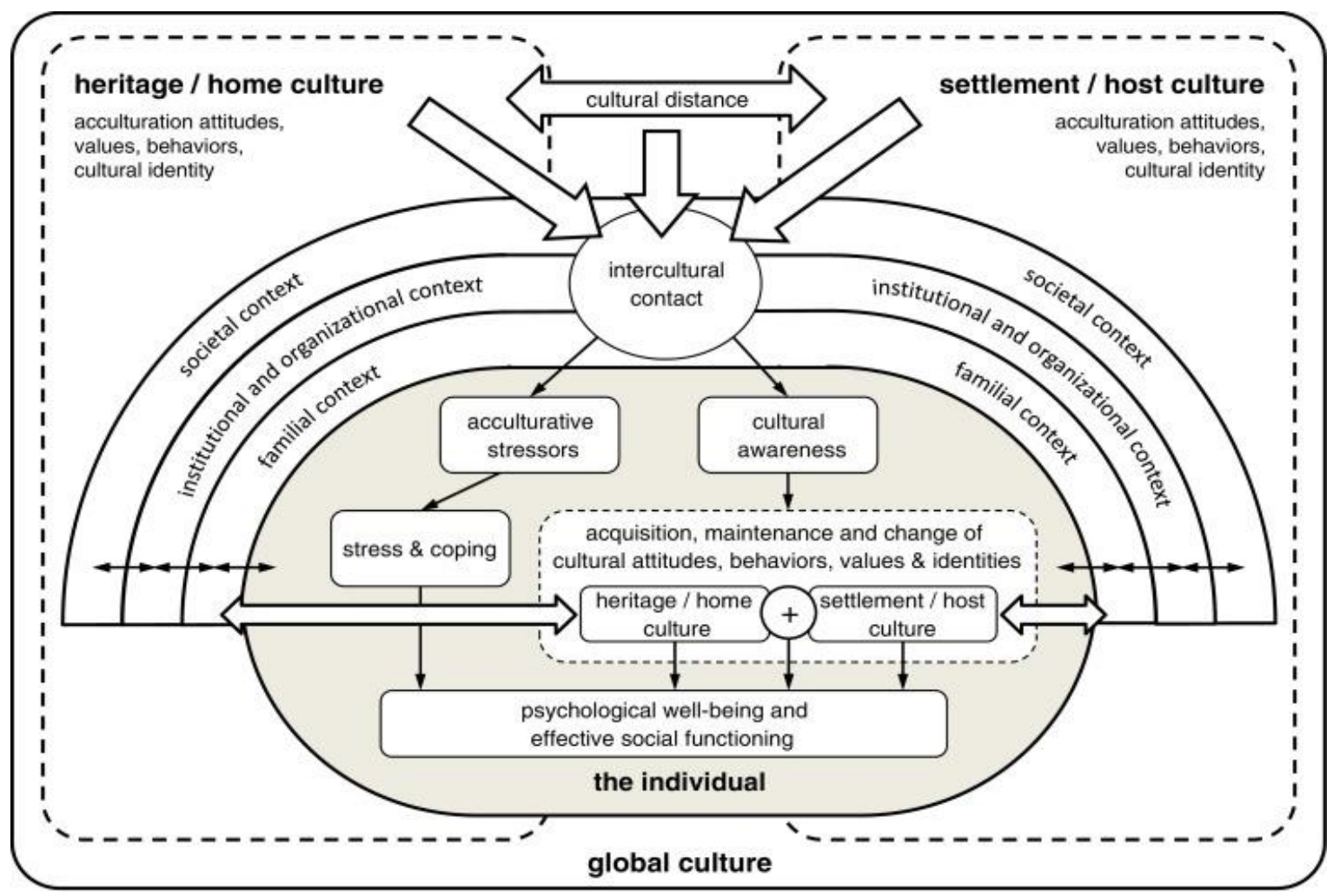

Figure 2.5. Acculturation process and context (Ward \& Geeraert, 2016, p. 99) 
This model has implications for this study. By focusing on the ecological context, especially institutional and familial contexts, the model recognises the roles of such contexts in international students' experiences and suggests that what they encounter in academic settings and familial factors are likely to impact on their experiences in host countries.

\section{N. Tran's (2011) Vietnamese-specific acculturation framework}

A later model of acculturation has been proposed by L. N. Tran (2011) to apply to Vietnamese international students, based on the findings from her doctoral study on the acculturation experiences of Vietnamese students in Australia (see Figure 2.6). The study drew on Berry's (1997) acculturation framework and De Jong and Fawcett's (1981) valueexpectancy model. The original version of this framework, which was initially based on a review of the literature on Vietnamese international students, was enhanced using the results of her study. Factors that existed in the literature may include demographic factors, socio-political-economic conditions, multiculturalism ideology, ethnic attitudes, education, gender, cultural distance, phrase of acculturation, social support and societal attitudes.

Tran added a number of new elements into his framework, including acculturation dilemma, cyber integration and vicarious acculturation, cultural mistrust, intercultural isolation and double discrimination. For L. N. Tran (2011), an acculturation dilemma was the mismatch between individuals' acculturation attitudes and their behaviours, and might result from a range of factors, such as the students' perceived hatred from Vietnamese immigrants and the distance between Vietnamese and Australian cultures. Cyber integration or vicarious acculturation referred to individuals' coping strategy in cyberspace. This strategy was associated with their desire for integration, and in cyberspace, they had online friends and interactions. Cultural mistrust related to the suspicion of Vietnamese students towards other international students and locals and might result from their subjective perceptions of other people. This type of mistrust might lead to an acculturation dilemma. Intercultural isolation related to a type of isolation triggered by their limited interactions with people from other cultural backgrounds, language difficulties, and racism. Double discrimination referred to a type of discrimination the students encounter from both the host who were Vietnamese immigrants and the hosts who were locals. Discrimination from Vietnamese immigrants was associated with the socio-political history of Vietnam where hundreds of 
thousands of the Vietnamese left the country as immigrants or refugees and sought to settle in other countries after 1975.

To date, few efforts have been made to develop an acculturation framework for Vietnamese international students. In that context, L. N. Tran's (2011) study is a valuable one. The model recognises the critical role of Vietnamese history and its possible impact on students' experiences and Vietnamese immigrant communities' attitudes towards international students. The new concepts of cultural mistrust and acculturation dilemma are useful because little has been known about these aspects of Vietnamese students' experiences.

Despite such advantages, sociocultural differences between Australia and New Zealand potentially affect the applicability of this model in the New Zealand context. Australia has a larger Vietnamese community than New Zealand. Also, in her model, L. N. Tran highlights Vietnamese immigrant communities' attitudes towards Vietnamese international students in relation to anti-communist attitudes, which may not be the case in New Zealand. 


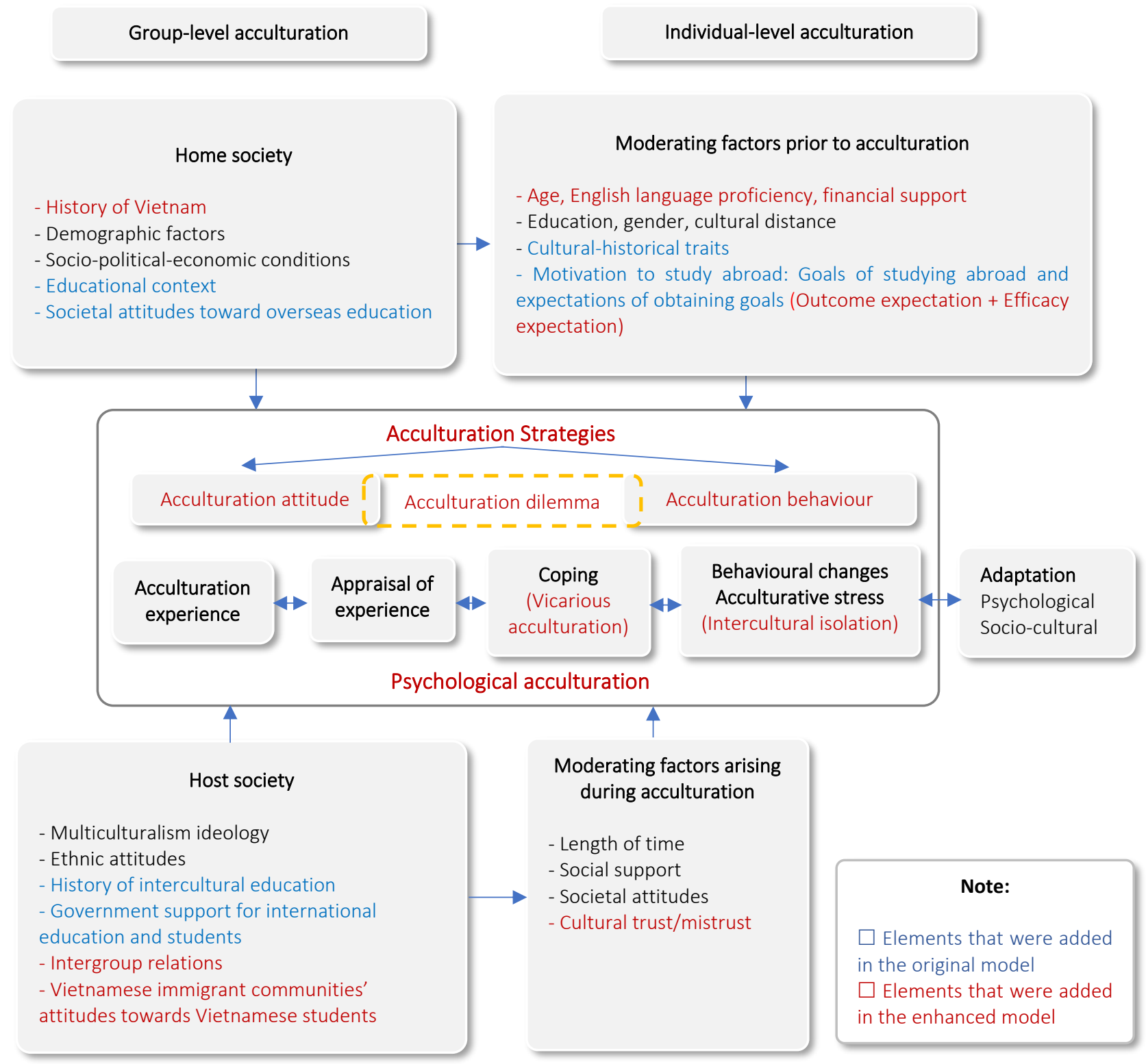

Figure 2.6. A Vietnamese-specific psychological acculturation framework (L. N. Tran, 2011, p. 272)

To conclude, whilst focusing on different groups of populations (e.g., immigrants and international students), the four acculturation models by Ward et al. (2001; 2016), Zhou et al. (2008) and L. N. Tran (2011) give an idea about how acculturation models have evolved and what acculturation may be like for different groups of individuals, including international students. Berry's model of acculturation framework has been modified with a range of elements being added to explain or clarify certain concepts, which are particularly relevant to the research on the experience of Vietnamese international students. 


\section{Marginson and Colleagues' Work on International Student' Experiences}

In this section, I will present Marginson's critique of acculturative studies on international students' experiences, describe his theory of international students' self-formation (2014), and introduce Marginson and colleagues' ideas around international student security (Marginson et al., 2010). While the two theories have different foci, both are closely related and have international students at their centre.

\section{Marginson's (2014) theory of international students' self-formation}

Marginson is a higher education scholar whose work on international education spans policy, sociology, and political economy. His theory of international students' self-formation (Marginson, 2014) can be seen as a response to the adjustment paradigm in psychology in which Berry's acculturation model is amongst the dominant theories.

Marginson et al. (Marginson, 2014; Marginson et al., 2010) argued that the term 'adjustment' implies one-way change and cultural superiority. It positions international students as needing to change rather than the host cultural and educational environments. The assumption is that international students will set aside "core elements of their identity" to integrate or adopt local cultural norms (Marginson et al., 2010, p. 391). Within an adjustment discourse, international students are conceptualised as being habitually weak and deficient. Marginson et al. (2010) counter this discourse by arguing that international students are often flexible in dealing with difficulties and able to combine home and host cultures without having to sacrifice their own culture. They emphasise the need for two-way processes and suggest that cross-cultural relations ideally involve mutual learning and change. In that context, Marginson et al. highlight international students' ability to reflexively manage their lives and form their self-trajectories or identities. They allow their home country identity to evolve along the way.

In international education, self-formation involves international students' substantial personal changes (Marginson, 2014; Marginson et al., 2010) and is "culturally reciprocal and culturally separated" (Marginson et al., 2010, p. 393). It is reciprocal when it leads to 
interaction between international students and locals, which may entail changes from both parties. While international and local students engage in self-formation (Marginson, 2014), their transformation takes place separately. International education contributes more to the self-formation of international students than to that of most local students, at least in Anglophone countries.

The notions of identity and agency are central to Marginson's view of international education as self-formation. Although Marginson believes that "all human relations are socially constructed and limited" (Marginson, 2014, p. 10), he recognizes that international students are autonomous and open to changes in the process of self-formation. They are able to choose what elements they want to include in their identities. Marginson (2014) subscribes to Sen's (1999) notion of multiple identities, which suggests that identities are associated with a range of factors (e.g., citizenship, religion, class, gender, and kinship). Certain identities are more stable than others, and thus, are unlikely to change, for example, familial relations, cultural identity, and national identity (Marginson, 2014).

The second critical notion in Marginson's (2014) self-formation is agency, "the sum of a person's capacity to act on her/his own behalf" (Marginson, 2014, p. 10). Undertaking education in a foreign country, international students receive identity resources from host countries besides those from their home country, which potentially leads to their negotiation of multiple identities. Self-formation may take the forms of educational credentials, language development, and social networks. Individuals may or may not choose the way they form their identities, but their agency is not affected. Another notion related to agency is agency freedom, which can be understood as the active human will that "guides reflexive self-formation and the self-negotiation of identity" (Marginson, 2014, p. 11). Drawing on Sen's perspective on agency, which emphasises the role of "responsible adults" who "must be in charge of their own well-being" (Sen, 2000, p. 288), Marginson (2014) highlights the pivotal role of agency freedom in international students' self-formation.

In the literature on international students' experiences, agency has been an important topic and can take several forms. Life-course and identity approach to agency (Biesta, Priestley, \& Robinson, 2015; Biesta \& Tedder, 2007; Emirbayer \& Mische, 1998) is usually used by 
researchers. M. N. Nguyen and Robertson (2020) found that Vietnamese doctoral students in Australia displayed relational agency. Exploring the relationships between international students' agency and their experiences in an Australian study, L. T. Tran and Vu (2018) found that the participants exercised collective agency for contestation. In both studies, the authors identified three forms of agency constructions: agency for becoming, agency as struggle and resistance, and needs-response agency. Similarly, exploring the agency of international graduates who were seeking jobs in Australia, L. T. Tran, Phan, Tan, and Rahimi (2020) identified two forms of agency: needs-response agency and agency as becoming. These findings collectively show that international students potentially enact a high level of agency within and outside academic settings.

Apart from agency as an important factor in students' self-formation, international students use two strategies in the process of self-formation - multiplicity and hybridity (Marginson, 2014). It involves multiplicity because an international student is "more than one person and lives more than one kind of life" (Marginson et al., 2010, p. 439). They may choose to maintain cultural elements from their home countries while absorbing new cultural values in host countries. Multiplicity is associated with dividing. In terms of hybridity, international students integrate or combine various cultural and relational elements into a new self. Some international students may adopt elements of both strategies.

\section{Marginson et al.'s (2010) concept of international student security}

Besides his work on student self-formation that conceptualises international students as agentic beings, this conceptualisation can be seen in his earlier ground-breaking work 'International student security' (Marginson et al., 2010). Marginson and colleagues have worked extensively on international students' security (Forbes-Mewett, Marginson, Nyland, Ramia, \& Sawir, 2009; Marginson et al., 2010; Marginson \& Sawir, 2011; Nyland et al., 2009; Sawir, Marginson, Deumert, Nyland, \& Ramia, 2008; Sawir, Marginson, Forbes-Mewett, Nyland, \& Ramia, 2012; Sawir et al., 2009b). Marginson (2012) notes that international students stay in a 'grey zone' of regulation. They are affected by regulatory regimes of their home and host countries, but fully covered by none, resulting in their vulnerability and uncertainty. Their non-citizen status affects their human rights, security, and capabilities. International student security is related to all aspects of students' academic and daily life. 
Marginson et al. (2010; 2011) draw on the idea of international student security to conceptualise the experience of international students, organising related issues into two major domains. In the formal and public domain, international student security includes, but is not limited to, finances, work, housing, health, personal safety, and the immigration department. In the informal and private domain, it is associated with their universities, language, family and friends, loneliness, and intercultural relations.

International students' security is associated with the idea of human security (Marginson et al., 2010). Partly shaped by the notion of human security of the United Nations (1948) and human agency by Sen (2000), human security can be understood as "maintenance of a stable capacity for self-determining human agency" (Marginson et al., 2010, p. 60). This definition of human security has two main elements - the protection of persons and their capacity to act. This notion of human security positions international students as active selfdetermining agents, although they may be affected by the external conditions of life. Undertaking education in another country could lead international students to lose the social protection regimes that they have in their home countries (Sawir et al., 2009b). However, with this conceptualisation, international students are viewed as active agents and bearers of full human rights (Sawir et al., 2012).

\section{Critique of Marginson's (2014) idea of international students' self- formation and Marginson et al.'s (2010) conceptualisation of international student security}

The advantage of Marginson's theory of self-formation is that it views international education experience as a process of self-development and self-cultivation and realises international students' agency. As such, this theory highlights the positive aspects of students' experiences rather than focusing on problems facing them as in the adjustment paradigm (Marginson, 2014), countering Berry's acculturation model. In the current study, I view students' agency as playing a decisive part in students' experiences. By adopting Marginson's self-formation theory, my project takes a student-centred perspective, and highlights Vietnamese students' agency and identity in the process of self-development. 
The strength of Marginson's international student security idea is that by drawing on the notion of human security, it recognises international students as having full human rights. It calls for recognition and protection of the human and quasi citizen rights of international students in host countries despite their non-citizen status. Marginson's concept of international student security is comprehensive because it relates to aspects of international students' experiences in both academic and everyday settings.

Both self-formation and human security conceptualise international students as agentic beings. Within this international-students-as-agentic-beings paradigm, students are viewed as having the power and capacity to choose what they think is suitable for them, achieve their goals, and contribute to home and host communities. In recognising students' agency, the two theories have shifted the positioning of international students from being passive individuals with problems (as in the adjustment paradigm) to active problem-solvers and choice-makers.

Marginson's ideas of self-formation and human security in the context of international education offer supplementary perspectives about students' experiences that Berry's acculturation framework pays limited attention to (i.e., students' agency). With such advantages, they have the potential to provide a better understanding of international students' experiences.

\section{Maslow's (1970) Hierarchy of Needs}

Besides Berry's acculturation theory and Marginson's theory of self-formation, I adopted a fourth theory - Abraham Maslow's hierarchy of needs - as part of the framework for the study. Maslow (1908-1970) was a psychologist whose work focuses on humanistic psychology and human motivations. His hierarchy of needs, which is a motivational theory in psychology, is one of the best-known psychological theories. This theory was appropriate for my study because it helps to understand how the housing-related issues might impact on the Vietnamese students' experiences from a psychological standpoint.

Maslow's hierarchy of needs can be visualised as a pyramid, with the needs being arranged in a hierarchical order (see Figure 2.7). The most basic needs are located at the bottom and 
move up to other needs at higher levels, including physiological needs, safety needs, belongingness and love needs, self-esteem and self-actualisation. These can also be arranged into three major groups: basic needs (physiological and safety), psychological needs (belongingness and esteem) and self-fulfilment needs (self-actualisation). Lower-level needs usually need to be met before higher-order ones can be achieved.

Physiological needs include fundamental requirements for survival (e.g., shelter or accommodation). If these needs are not satisfied, people cannot function properly. For example, if people suffer from hunger, they may generally resort to available resources to acquire food and cut out other less important needs. Therefore, physiological needs are considered as the most basic and take priority over others. The safety needs become predominant once the physiological needs are met. As the name suggests, the safety needs involve security and safety, and are associated with such issues as law, order, financial stability, personal security, good health, and freedom from fear.

Belongingness and love needs, or social needs, emerge after the physiological and safety needs are satisfied. Arriving in this stage, people begin to consider and attend to companionship and community, wanting to give and receive affection and love. According to Maslow (1954), people generally want to have self-respect and respect from others. There are two kinds of esteem needs. The first one may include self-esteem and confidence, the desire for personal achievements and independence. The second one may consist of reputation, prestige and status, and the desire for people's admiration.

The highest level of the hierarchy of needs is about a desire to realise personal potential and seek personal development. This need motivates an individual "to become everything one is capable of becoming" (Maslow, 1987, p. 64). This theory suggests that people encounter more difficulties in reaching higher need levels. Therefore, fewer needs at the upper levels of the pyramid are met compared to the lower levels, and few people can fully reach the level of self-actualisation (Maslow, 1954). 


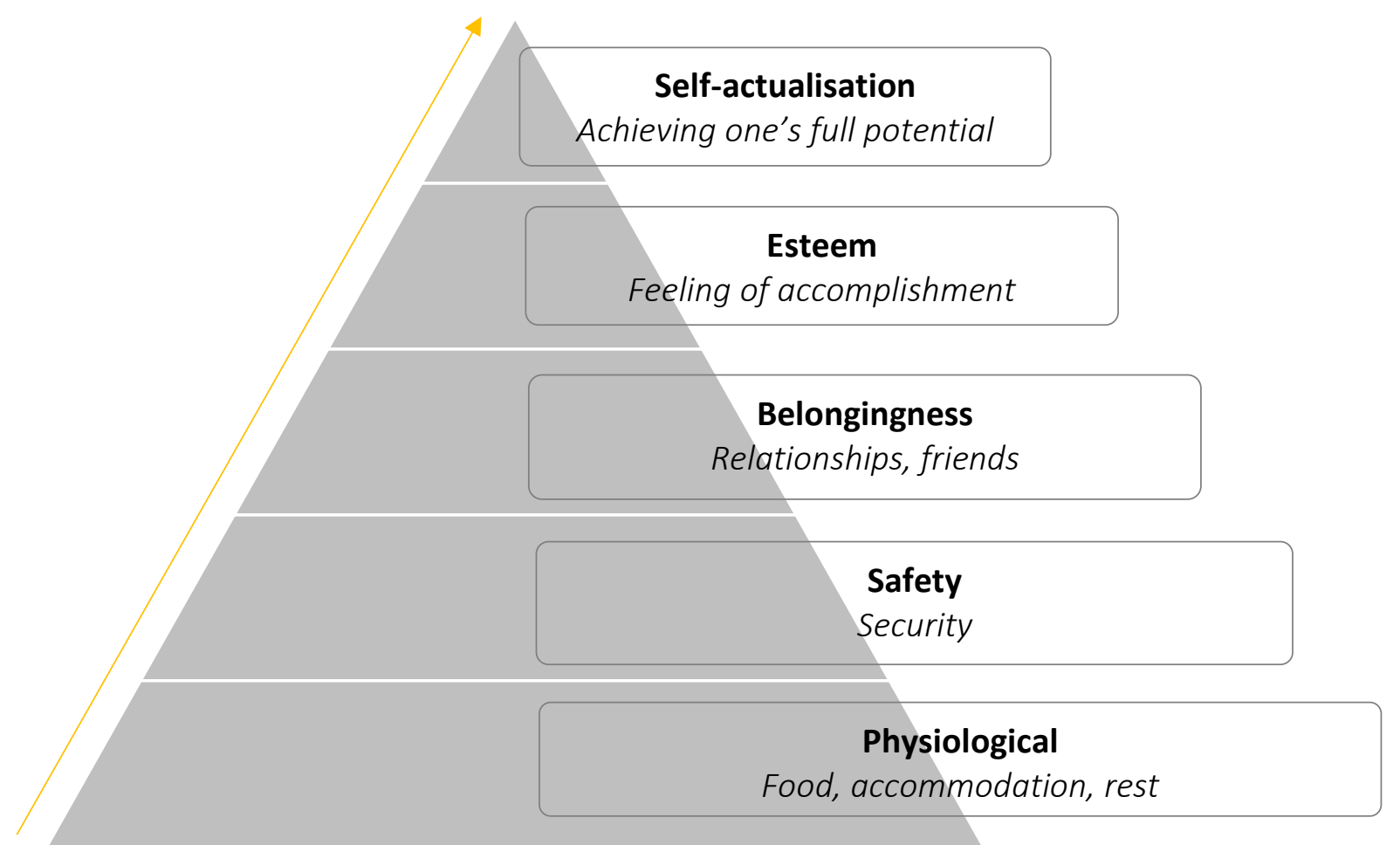

Figure 2.7. Maslow's hierarchy of needs, adapted from Maslow (1970)

Maslow (1954) maintained that there might not be a clear-cut boundary between satisfaction and dissatisfaction within a need level. Most people may experience different levels of satisfaction (and dissatisfaction) in all need levels at the same time. He proposed that people's behaviour may be driven by many factors, saying that "any behaviour tends to be determined by several or all of the basic needs simultaneously rather than by only one of them" (Maslow, 1987, p. 71).

\section{Critique of Maslow's hierarchy of needs}

Past research has focused on the role language, academic, social, and emotional factors play in international student experiences. In recent years, attention is beginning to be placed on accommodation and security issues. Such issues correspond with the lower levels of Maslow's (1954) hierarchy of needs. Although the hierarchy of needs comes from the psychological field, researchers have recognised its applicability to education (Alexander, 2005; Schunk, Meece, \& Pintrich, 2012). This theory is useful to educational researchers in achieving their aims of facilitating learning and making this process meaningful and useful to learners' lives. Theories of learning and motivation derived from Maslow's hierarchy 
position the meeting of basic needs for food, shelter, safety, and belonging as essential precursors for learning and self-actualisation (Schunk, 2012; Schunk et al., 2012). Limited satisfaction of students' basic needs (i.e., physiological and safety) may inhibit the learning process because they may give greater attention to these needs rather than their studies. Within the context of cross-national education, distance from their networks potentially poses challenges to international students in terms of satisfying their basic needs. As a result, learning outcomes and self-development will become less important.

There are similarities and differences between the notions of human security (Marginson et al., 2010) and self-formation (Marginson, 2014) and Maslow's (1970) hierarchy of needs. Similar to Marginson and colleagues' idea on international student security, Maslow's theory recognises the importance of meeting basic needs (e.g., accommodation, security, belongingness, and friendship). However, rather than conceptualising people's (or international students) experiences as including a range of interrelated aspects like in Marginson and colleagues' work, Maslow focuses on individuals' needs, arranges them in an order and suggests that the basic needs (e.g., needs for shelter or security) have to be satisfied before an individual can achieve higher-level needs. Besides, in Maslow's theory, security is positioned at the safety level and relates to personal security, order, and stability while in Marginson et al.'s work, the notion of human security encompasses all aspects of international students' experiences (e.g., accommodation, social networks and education). Finally, Marginson's notion of self-formation encompasses a range of changes which result from overseas education and relate to students' identities, educational credentials, and selfimprovement while Maslow's need for self-actualisation relates to one's desire for achieving their full potential and capacities. In this regard, self-formation can be regarded as part of international students' efforts to obtain self-actualisation. It is a more contemporary rendering of self-actualisation and links to a greater emphasis on identity.

Maslow's theory is simple and easy to apply, which may have resulted in its wide application (Fallatah \& Syed, 2018). However, the theory is not without disadvantages, one of which is its focus on the individual rather than the collective. The theory draws on a Western individualist ideology and the arrangement of needs as proposed by Maslow may differ for individuals from different backgrounds (Fallatah \& Syed, 2018). Maslow worked on refining 
his theory for decades (Maslow, 1943, 1962, 1987) and noted that the order of the needs "is not nearly as rigid" (Maslow, 1987, p. 68) as he had proposed in his early work. Gambrel and Cianci (2003) reviewed the literature and suggested that a new hierarchy of needs for a collectivist culture may be needed with the basic need being belonging, self-esteem being removed, and self-actualisation being achieved by satisfying societal development needs.

The theory focuses on individuals in everyday contexts and fails to account for the fact that in different situations, individuals may have different priorities, which potentially affects their needs (Schunk, 2012). For example, people may risk their lives to rescue others from danger. In such situations, lower-order needs are not always favoured over higher-order needs. This is consistent with the idea of Gambrel and Cianci (2003) who criticised the theory for being too simplistic and overlooking societal needs at a particular time (e.g., economic crisis or war). Across a sample of 123 countries, Tay and Diener (2011) concluded that people in different age groups might rank their needs differently.

Finally, Schunk (2012) criticises the theory for its conceptual vagueness, positing that Maslow did not clearly define a deficiency. What is considered as a deficiency in one society may not be in another society. Besides, the qualities of self-actualised individuals are varied, and self-actualisation can take many forms. Research has yielded mixed results about how it may appear and can be influenced.

\section{Theoretical Framework of the Study}

In this section, I present how I developed a theoretical framework to explore the experience of Vietnamese students in New Zealand by incorporating four theories: Berry's acculturation framework, Marginson's conceptualisation of international student self-formation, Marginson and colleagues's idea of security, and Maslow's hierarchy of needs. I also explain how I conceptualised the relationships between the theories.

Cultural contact is at the centre of Berry's acculturation framework. In this study, factors in both home and host countries that contribute to the acculturation process are explored. Host country factors included issues relating to academic and everyday settings. Home country elements may include cultural values, students' prior lives, and academic 
experiences. I consider a range of factors at the individual levels, such as language proficiency, prior overseas experiences, and perceived cultural differences. The acculturation strategies are positioned in relation to home and host country factors. I noted from the literature that marginalisation has been rarely reported. However, I was open to exploring this acculturation strategy. Rather than removing it from the model, I depicted it with a dotted line and question mark.

Agency is an important contributor to international students' experiences, but Berry's framework pays insufficient attention to this element. In this regard, Marginson et al.'s idea of human agency offers a means to capture students' capability to enact their agency in new and unfamiliar settings. Student self-formation provides a conceptual basis for exploring how students construct and reconstruct their identities through overseas education. Students' agency, identity and needs are individual factors that emerge in the process of acculturation and are partly affected by home and host factors. I theorised that their identity is more likely to be associated with home country factors and cultural values while their needs and security would be more related to host country factors. In utilising Maslow's and Marginson et al.'s work, I placed emphasis on human needs and security.

Although each of these theories is distinct, there are overlaps among them. Human agency is at the centre of Marginson's ideas of human identity and Marginson et al.'s conceptualisation of international student security. Maslow's conceptualisation of needs is closely related to Marginson's idea of student security.

The acculturation process is complicated and encompasses a wide range of factors, including students' needs, agency, identity, and security. On that basis, my conceptualisation is that student acculturation experiences are affected by all these factors.

The four theories identify a number of key issues in international students' experiences and loosely provided the topics to be explored in the literature and in the interviews. The theoretical model of this study is presented in Figure 2.8. 


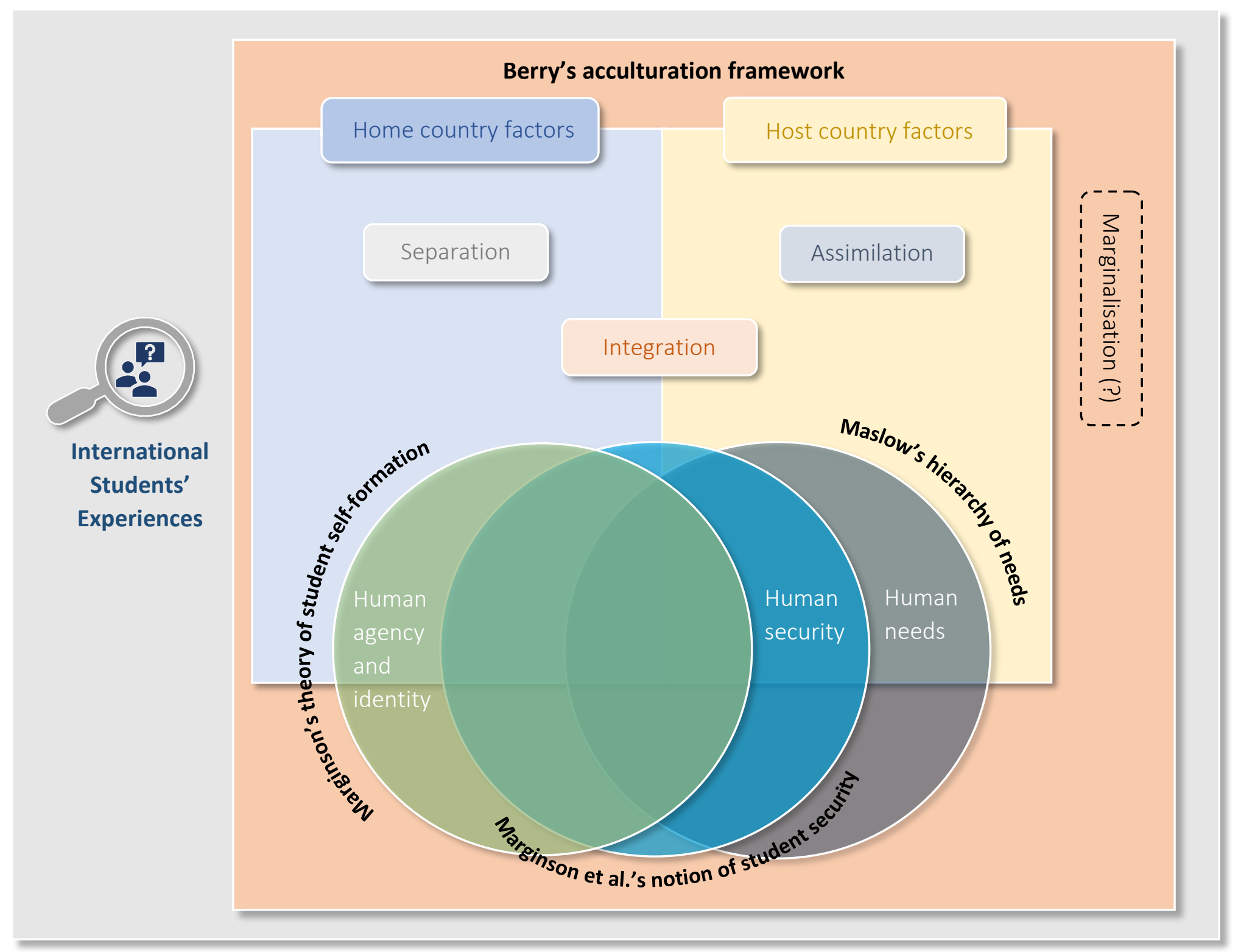

Figure 2.8. Acculturation framework for international students 


\section{Why Were Western Theories and a Western Research Approach Used Rather Than Vietnamese Indigenous Theories and Ways of Knowing?}

I was challenged as to why I drew on Western theory, philosophy, and research approaches rather than looking to Vietnam for these. In this section, I outline my rationale for choosing to frame my study using Western theoretical and methodological approaches rather than intellectual traditions that are indigenous to Vietnam or the South-East Asian region.

Contemporary Vietnam culture and society incorporates or has vestiges of values and beliefs from ancient and modern times that originated locally and/or from other countries (Q. T. N. Nguyen, 2016). 'Tam giáo' (Three Teachings or Triple Religions) is the term used to refer to the mix of prominent religious philosophies in Vietnam, including Buddhism, Confucianism and Taoism/Daoism (Vuong et al., 2018). However, in Vietnamese modern society, Taoism has little influence. This religion is evident in such shaman-like practices as thầy bùa (masters of the amulets), thầy pháp (magician healers), and thầy bói (fortune tellers/soothsayers), which are viewed as irrational by many Vietnamese people (A. Q. Tran, 2017).

Confucianism has had long-lasting influence on Vietnamese culture. However, Vietnamese society is changing rapidly, and something true a few years ago may not be true any longer. This perhaps is the case of Confucian values. That a considerable number of researchers frame their studies on aspects of Vietnamese modern society (including education) drawing on ideas from Confucianism, for example, Truong, Hallinger, and Sanga (2017), Selvarajah and Meyer (2020) and Tho (2016) may give an impression that Confucian beliefs are still dominant in Vietnamese modern society and things have remained almost unchanged over the years. This may be misleading. The changes in Vietnamese cultural systems have happened over a long period of time and have been depicted in the Vietnamese literature. The poem 'Ông đồ già' (The old Sino-Vietnamese teacher) taught in high schools is known by many Vietnamese people. It was written by Vũ Đình Liên in 1936. It describes the nostalgic feelings of the poet about the good old days when seeing Vietnamese society changing and many old values (including those relating to Confucianism) being altered. Some verses are as follows: 
Ông đồ vẫn ngồi đấy,

Qua đường không ai hay.

Lá vàng rơi trên giấy

Ngoài trời mưa bụi bay.

Năm nay đào lại nở

Không thấy ông đồ xưa.

Những người muôn năm cũ

Hồn ở đâu bây giờ?
The teacher is still sitting there,

Pedestrians are unaware.

A yellow leaf falls on the paper

As drizzle flies in the air.

This year, peach trees bloom again

The ancient teacher is not seen.

People from millennia past

Where are your souls now?

(Translated by Oanh Nguyen)

The 'Doi Moi' economic reforms have brought about remarkable and profound changes in Vietnamese society (T. Nguyen \& Angelique, 2017). These changes have given rise to new cultural values and beliefs which are different from or are in conflict with the Confucian doctrines and principles (T. Nguyen \& Angelique, 2017; Pond, 2014). In discussing the current sociocultural situations of Vietnam and its impacts on the young generations in Vietnam (those who he has defined as under 29), Pond (2014) comments:

Theirs is the first generation that has had to invent its own life instead of simply replicating its elders' subsistence farming or guerrilla privation. They are the first in their families to have chosen their own marriage partners rather than accepting a union arranged by parents. They are the first generation to adopt the custom of bringing the toilet from the outhouse into the middle of the home. They are the first to give their toddlers tricycles and blow-up plastic pools to splash in and let them watch Tom and Jerry cartoons on TV... They are the first generation in five millennia to have known only peace in their lifetime.

In this excerpt, Pond touches upon some attributes of many young Vietnamese, who are highly dynamic. In modern society, young Vietnamese usually proactively create their own image and identity (D. H. Doan, 2005). These young people have the freedom to decide on their own lives and in some situations, do not feel a need to follow the pre-existing conventions or pathway. Many enjoy better living conditions than their parents and 
grandparents, and most are allowed to decide on when and who to marry rather than relying on their parents for their arrangements - this is something that is clearly against the Confucianism and feudalistic values that their elders abided by. This suggests that there are aspects of Confucianism that Vietnamese young people are likely to find inappropriate and consider as belonging to the old days. Many young Vietnamese favour and adopt aspects of Western lifestyles and values, and live in an independent country for the first time after thousands of years under the Chinese domination and a hundred years of French and American colonisation/regimes. These have contributed to the identity of the young Vietnamese generation.

Vietnam has its own folk or indigenous religions, many of which have ancient origins, and typically involve worshiping ancestors, historical figures (e.g., national heroes and heroines), supernatural forces (e.g., local gods and goddesses/deities), local festivals honouring village gods, practicing divination and forms of exorcism of evil spirits, making offerings to gods and goddesses for blessings and protection (A. Q. Tran, 2017; Vuong et al., 2018). Cao Đài (Caodaism, which combines Buddhism, Christianity, Taoism, Confucianism, and Islam) and Phật Giáo Hòa Hảo (Hoahaoism or Hoa Hao Buddhism, which is a variant of Buddhism) were established during the nineteenth century (The United States - Department of Justice, 2020; U.S. Embassy and Consulate in Vietnam, 2010) under the French colonial period. These two relatively new indigenous and syncretism religions are mainly practiced by people in Southwestern Vietnam (or the Mekong River Delta).

Interestingly, whilst there are rich intellectual and philosophical traditions in Vietnamese culture and society, figures indicate that over $80 \%$ of the Vietnamese identify themselves as atheists (Indexmundi, 2019). Most of the participants of my study were in their twenties, might be atheists, and are likely to be familiar with Western values through their tertiary education in Vietnam and international education in Aotearoa-New Zealand. No participant was from the Mekong River Delta, where most followers of the Cao Đài and the Phật Giáo Hòa Hảo are from. I acknowledge that philosophical traditions can offer an expression of selfhood; a way of living in the world and understanding how it is structured, and express the worldviews shaped by the people of that region and are indigenous to them. However, coupled with the early purposes of the Chinese authority's introduction of Confucianism to 
Vietnam being to foster cultural assimilation and to strengthen the Chinese domination in the nation as indicated earlier, the aforementioned considerations raise a concern whether such intellectual traditions could be used effectively as major theoretical approaches to frame this particular study or interpret Vietnamese students' experiences in international education, and whether students feel comfortable with their behaviours being interpreted in relation to these intellectual traditions. These considerations made me reluctant to rely heavily on such intellectual traditions, where I considered the participants' behaviours as being shaped by a range of factors, including international education, home country's cultural values, and students' agency.

\section{Chapter Summary}

In this chapter, I have described the four theories used to frame this study, including Berry's acculturation framework, Marginson's concept of self-formation, Marginson and colleagues' idea of international student security, and Maslow's hierarchy of needs. These theories provided additional interpretive lenses for my study. Berry's acculturation framework suggests that many factors contribute to international students' experiences (e.g., language difficulties, cultural differences, and social networks). Maslow's hierarchy of needs shows that human needs can be arranged in a hierarchical order and include different levels. This theory emphasises that basic needs such as accommodation and safety must be met before higher-level needs can be addressed and thus implies that if students have unpleasant experiences with their accommodation, they may not have a sense of belonging to their homes and their studies are likely to be affected. I have critiqued Berry's and Maslow's approaches. On that basis, I was careful not to adopt controversial aspects of the theories to my study or not to imply ideas that may be problematic.

In the following chapter, I outline a number of factors that may affect international students' experiences. I review studies on Vietnamese students' experiences and address the gap in the literature. 


\section{Chapter 3 | Literature Review}

In this chapter, I review the literature on factors that shape international students' experiences in a host country. I introduce a number of challenges facing them in the academic and everyday settings and how they deal with difficulties. Finally, I review studies that focus specifically on Vietnamese students' experiences, highlighting the gaps in the literature and outlining the contributions my study makes to the field.

\section{The Experience of International Students}

Many students studying in another country experience distress when settling in a new and unfamiliar environment. Moving to foreign countries and leaving behind family and social networks may present a range of difficulties (Ward et al., 2001). International students' experiences are complex and unique. While local students may encounter the same difficulties in many aspects of their lives (e.g., accommodation, finance, and loneliness), many unique factors may contribute to and exacerbate international students' experience (e.g., language barrier, absence of networks, and discrimination). Students may be affected by a range of academic, social and psychological factors (C. Wang, Whitehead, \& Bayes, 2017). Coming from all walks of life with various cultural values and beliefs, international students arrive with diverse expectations, aspirations, concerns, and goals.

There are different ways of categorising international students' experiences in the literature. In this study, student experiences are divided into two different but interrelated groups: living and academic experiences. In the following sections, I present the challenges that international students may face in academic and everyday settings.

\section{Academic experiences of international students}

Academic experiences refer to what students experience in educational settings. While studying in a foreign country, many students go through a challenging process to get accustomed to a new learning environment. Learning environments include the physical and 
pedagogical context where learning occurs. In this study, I mainly use this term to refer to formal learning in academic contexts.

Unfamiliar teaching and learning methods and language issues potentially lead to difficulties in academic experiences (Z. Yan, 2020). Other issues are related to the curriculum structure, minimum credit hour requirements, heavy workload, academic competence, high expectations, presentations, writing skills and differences in assessment (Cowley \& HyamsSsekasi, 2018; Heng, 2018; H. Li, 2019; J. Li et al., 2018; Naylor, Chakravarti, \& Baik, 2018). Reviewing the academic experience of international students in North America, C. Smith (2020) postulates that challenges facing them in the classroom may include exclusion from group discussions and adjustment to a new educational system.

International students may find English academic writing conventions unfamiliar. Exploring the adjustment experience of Indian students in New Zealand, Kukatlapalli et al. (2020) found that some students had problems related to paraphrasing, referencing, avoiding plagiarism, writing critically, structuring and expressing ideas, and forming argumentation. International students' perceptions of plagiarism may be linked to low language competence or differing cultural understandings of citation and referencing, for example, viewing paraphrasing texts as a 'disgraceful act' and a lack of respect for the author (C. Smith, 2020).

There may be differences or gaps between what students know or experience in their home and host countries, which may result in undesired consequences. In Mukminin's (2019) study on Indonesian students in the Netherlands, the students reported having inadequate knowledge of the topics discussed in class due to mismatches between home and host countries' teaching content. This contributed to their stress and limited engagement in discussions. Similarly, H. Li (2019) suggests that Chinese students in her study experienced differences in curriculum content and structure between Chinese and German universities, and difficulties understanding the content of teaching. Some failed and had to repeat the course. Kukatlapalli et al. (2020) found that some of the Indian student participants had limited experience or knowledge of the academic language and writing requirements before they began their studies in New Zealand. Similarly, H. Li and Pitkänen (2018) referred to 
differences in 'intellectual background' such as teaching methods, which affect students' understanding of teaching content. Although they studied under bilateral agreements between Finnish and Chinese universities, the course content was not synchronised between the two universities. Therefore, the students needed instructions from teachers to facilitate their understanding of course content.

H. Li (2019) and H. Li and Pitkänen (2018) found that 'pedagogical differences' contributed to the Chinese students' learning difficulties and limited participation in class discussion. Having been taught to respect the views of authority from childhood in their home country, they might feel uncomfortable questioning and challenging peers and lecturers. They would not join discussions or group assignments because they were unfamiliar with those practices. A Western debate culture appeared to be unfamiliar to them. For fear of giving wrong answers to questions and being ashamed, they were reluctant to articulate what they thought in class. H. Li and Pitkänen (2018) explained that in China, students typically focused on memorising course content and repeating it in tests or exams. Meanwhile, in Finland, lecturers highlighted the importance of understanding subject content, being independent learners, and thinking critically. H. Li (2019) noted that some students would rather be quiet and not give answers in class until they understand more about the teaching content. She acknowledged that although cultural beliefs may explain their silence, personal characteristics, such as shyness and modesty, may also play a role.

H. Li and Pitkänen (2018) suggested that differences between Finnish and Chinese pedagogical contexts may pose challenges to international students and contribute to their learning experiences. In Finland, lecturers appeared to be flexible while in China, each class had a monitor who helped students with their learning issues. Self-directed learning was encouraged in Finland, and lecturers expected their students to be independent learners. Similarly, Mukminin (2019) highlighted unfamiliar classroom environments and heavy workloads as potential stressors for students.

It is important to avoid stereotypes when examining international students' learning experiences. In a study on Chinese students' learning experiences in British universities, Wu (2015) emphasised that students' experiences are affected by multiple factors (e.g., cultural 
and academic contexts, and students' intellectual development) and may change in different environments. Students may be passive learners in one environment but become active ones in another environment. Thus, it is advisable to examine students' learning experiences in the settings where they are.

\section{Everyday experiences of international students}

In the everyday setting, international students encounter a range of issues related to finances, accommodation, support networks, language difficulties, loneliness, and isolation (Deumert, Marginson, Nyland, Ramia, \& Sawir, 2005; Forbes-Mewett, 2018; Marginson et al., 2010). These issues are considered in the following sections.

\section{Pre-departure preparation}

Once international students decide to study overseas and choose their destination countries, they often seek information about studying and living in the new country. Good preparation is usually associated with positive experiences. Information about food, culture, educational system, finances and accommodation are available to relieve students' anxiety (Bartram, 2008; Lamberton \& Ashton-Hay, 2015). Based on a review of the experiences of Chinese international students, Zhu (2016) emphasised that prior knowledge about host countries (e.g., norms, customs, and values) can facilitate students' adjustment. Conversely, a lack of pre-departure preparation may lead to difficulties. Insufficient prior knowledge of academic conventions could contribute to challenges in producing academic written work (e.g., assignments and reports). M. Chen and Bang (2020), exploring East Asian undergraduate students' perceptions of the impact of pre-departure preparation on academic success in the USA, found that proper preparation relating to language ability and knowledge of the host country culture contributed to positive academic experiences.

Investigating the role of readiness in the academic adaptation of international students in China, Hussain and Shen (2019) suggested that pre-departure preparedness helped establish readiness among international students. Those who were ready for university education were more likely to have positive learning experiences. Sources of pre-departure preparedness might include the international student office, family and friends, and home 
countries' national curriculum. Pre-departure information might include country laws, visa regulations, campus life, degree criterion, and academic environment. In his study on Japanese students' counterfactual understandings of a three-week short-term study at a Canadian university, Douglas (2019) found that the students wished they had had information about food, drink, facilities, and community characteristics. The food and beverage choices were different and not particularly healthy. The students wished they had known more about facilities (e.g., fitness facilities) on campus and in their local communities (e.g., public transport) before their arrival. Some had unpleasant experiences because of theft in the student residences and language barriers, i.e., their belongings being stolen and communication barriers. On reflection, they remarked that if they prepared differently, they might have had different experiences in Canada.

\section{Financial issues}

Personal finances are at the heart of international students' security (Marginson et al., 2010) and one of their key concerns both before and after their departure (Khanal \& Gaulee, 2019). Pre-departure financial concerns are linked with private loans, cost and return-oninvestment calculations, the fluctuation of currency. Post-departure financial concerns are associated with unreliable payment schedules in higher education institutions, tuition, and fees. International students, especially self-supporting and family-funded, are more likely to struggle with living and studying costs (Chien, 2020; Kuo, 2013).

Finance, while a potential concern for all students, is especially relevant for international students. A range of factors may prevent them from securing a job to cover expenses, which makes their experience unique. These include language barriers, employers' concerns about students' visa status and retention, lack of work experience in host countries, lack of jobs in certain fields, discrimination by employers (Coffey, Farivar, \& Cameron, 2018; Kukatlapalli et al., 2020) and limited access to work off-campus (C. Smith, 2020).

International students may experience intense financial pressures (Forbes-Mewett et al., 2009; Forbes-Mewett \& Sawyer, 2016; Kukatlapalli et al., 2020; C. Smith, 2020). A poor financial position may be associated with psychological distress (Neto, 2021). Families tend to be the main source of financial support for international students, followed by 
scholarships and paid work (Kukatlapalli et al., 2020). However, scholarships are a critical source of support but limited in number and may be hard to achieve (Qi \& Li, 2020). Therefore, many students work part-time employment not just to support themselves but to reduce the burden on their families (Qi \& Li, 2020).

\section{Accommodation issues}

Accommodation plays an important role in international students' experiences (Arkoudis, Dollinger, Baik, \& Patience, 2019; Marginson et al., 2010). Rising numbers of international students entails a greater demand for rental accommodation. However, the housing markets in many host countries have been stretched and the availability of good quality and affordable housing may be limited (Fincher, Carter, Tombesi, Shaw, \& Martel, 2009).

Housing costs are strongly associated with students' financial situations. In an Australian study, Farbenblum et al. (2019) found that housing problems might substantially exacerbate the financial issues confronted by international students in Sydney. They provided the hypothetical example of a student moving out of accommodation, who is unable to get her $\$ 2000$ bond back, so is then unable to secure alternative accommodation. This may cause her to work more hours and result in reduced university attendance or failing a subject. Forbes-Mewett (2018) recognised that cost was the concern of many international students when they decided on where to live. However, low-cost housing might be associated with less personal security (e.g., a neighbourhood occupied by drug dealers). Therefore, students might prefer to stay close to their co-national peers to help with such security issues.

Accommodation is an important environmental factor because it may affect international students' security (Marginson et al., 2010), their social and academic experiences (Farbenblum et al., 2019; Judd, 2013; Long, 2014), and overall experiences in host countries (Ammigan, 2019; Farbenblum et al., 2019). Relation with flatmates and distance from campus may contribute to students' housing experiences (Calder et al., 2016). In a UK study (UCAS, 2018), location was rated as the most important factor in students' choice of accommodation. 
While domestic students may encounter similar problems, international students' experiences are unique because they lack the knowledge that many locals have (Marginson et al., 2010), and they are distant from their support networks (Lin \& Kingminghae, 2014; Sawir et al., 2008). This underlines the need for university accommodation offices to assist international students in finding and securing accommodation (Ammigan, 2019).

Negative housing experiences can affect students' mental and physical wellbeing (Farbenblum et al., 2019) and life satisfaction (Deloitte, 2008). Problems in settling in a foreign land (e.g., difficulties in finding private rental accommodation) could exacerbate students' feelings of loneliness (Sawir et al., 2008). Students with higher levels of satisfaction with their accommodation are more likely to report positive emotions (Ryan et al., 2016). In a UK survey carried out by The Universities and Colleges Admissions Service (UCAS, 2018), $63 \%$ of 70,000 student respondents rated the standard of their accommodation as 'very important' to their wellbeing. Farbenblum et al. (2019) linked higher distress with living in shared accommodation compared to living in a university hall or with family. Poor housing conditions, especially overcrowded houses that lack private spaces, can have a negative impact on students' physical health. Problems with living arrangements may lead to stress and sleep problems (Farbenblum et al., 2019). Students who feel uncomfortable in their houses are likely to spend less time at home. If they do not have a quiet and safe place to study or rest, their studies may suffer.

\section{Social connection and interaction}

A plethora of research has been conducted to explore the friendship formation of international students and how this network may impact on their experiences. Such issues remain a recurrent theme in the literature on international students (Marginson et al., 2010; Montgomery, 2010; Robinson et al., 2020).

Bochner et al. (1977) classified international students' friendship networks into three categories: co-national, host-national, and multi-national networks. Montgomery (2010) described the international students in her UK-based study as having complex friendship patterns involving a mixture of nationalities. Despite this friendship diversity, many reported that close friends were co-nationals. Although international students value and want to form 
relationships with both domestic and other international students, their closer connections are with co-national and other international students (Ammigan, 2019; H. Li, 2017). Examining the social network of international students in the USA, McFaul (2016) suggested that students may form different types of friendships in different settings. In class, they may have friends who are multinationals, co-nationals, and host-nationals. They also formed relationships with flatmates who were either locals or co-nationals. In all the studies, conational friendship tended to prevail.

There are several reasons for international students' strong relationships with co-nationals and other international students. Those individuals appear to be more open to meaningful social relationships (H. Li \& Pitkänen, 2018; Palmer, 2015; Rienties \& Nolan, 2014). Conational friends may provide useful information for daily life, and support with social, psychological, and academic issues (H. Li \& Pitkänen, 2018). By mixing with co-national friends, international students may be comforted by shared language, cultural heritage, and access to mutual assistance (Brown, 2009). Being international students themselves, conational friends are an important source of encouragement to each other.

There are multiple reasons for the rarity of international students' relationships with local students, including language barriers, lack of understanding due to cultural differences (Belford, 2017; H. Li \& Pitkänen, 2018), and limited opportunities for interactions (C. Wang et al., 2017). International students may need to invest time adjusting to unfamiliar learning environments, demanding subjects and heavy academic workloads, which reduces their time for social activities (H. Li, 2017). In their Canadian study, Robinson et al. (2020) found a range of constraints that hindered international students from having meaningful interactions with local students, including their concerns over the ways local students received them, cultural boundaries, and the students' foreign accent. The authors explained that the students were not ready to form meaningful relationships with local students because they perceived some behaviours as too costly for them, for example, the perception of the need to adjust or sacrifice their lifestyle, academic experiences, and values to adapt to the local culture. 
Social networks may impact on international students' experiences. There is an association between students' social networks and their contentment, decreased homesickness, and academic achievement in a host country (Bochner, Hutnik, \& Furnham, 1985; Kudo \& Simkin, 2003; C. Smith, 2020). Friendship networks may have various functions - affirming or expressing the culture of origin, facilitating academic aspirations, and being recreational (Bochner et al., 1977).

Meaningful relationships with local students may benefit international students in a number of ways. Quality contact with the host society may facilitate international students' crosscultural learning and personal development (Y. Yu \& Moskal, 2019). Friendship with local students might enrich international students' personal lives, and local students may help them improve their language proficiency and understanding of the host national culture $(\mathrm{H}$. $\mathrm{Li}$, 2017). Students with more local friends may feel more satisfied and less homesick (Hendrickson, Rosen, \& Aune, 2011). Few meaningful interactions with local students may contribute to students' feeling of not belonging (Glass \& Westmont, 2014; Palmer, 2015). Montgomery (2010), however, challenges the idea that international students need to form strong relationships with local students to have positive experiences in a host country, highlighting the ability of international students to help each other.

International students may gain good academic results without friendships with local students because they can seek assistance from their co-national peers to deal with academic demands (H. Li, 2017). Frequently communicating and interacting with other international students may improve language proficiency and cultural knowledge $(\mathrm{H}$. Li \& Pitkänen, 2018). Co-national friends may assist with adjustment issues, which had a positive impact on international students' well-being and helped to decrease the feeling of loneliness and disorientation (Brown, 2009; Montgomery, 2010).

\section{Language issues}

Many international students are from countries where English is a foreign or second language. Therefore, much of their choice of destinations has traditionally targeted the Anglophone countries because of the global status of English as a global language, and students' desire to develop their English language skills (Nogami, 2020). Many view 
improvements in their language ability as a benefit of overseas study (Chien, 2020). Although there are English language requirements for many universities (e.g., the IELTS test - International English Language Testing System), meeting the requirement does not guarantee students' ability to effectively deal with language issues in daily life and study (Elturki, Liu, Hjeltness, \& Hellmann, 2019).

The literature on international students emphasises the critical role of language in students' cross-cultural experiences. Language may pose challenges to students in both academic and non-academic settings (Alsahafi \& Shin, 2017; Andrade, 2006; Khanal \& Gaulee, 2019), which makes their experience unique compared to those of local students' (Education New Zealand, 2018a). Language difficulties relating to oral communication may result from unfamiliar accents, limited vocabulary, and pace of speech (Chien, 2020; J. Li et al., 2018; C. Smith, 2020).

In the academic setting, international students may need considerable time to become familiar with using a different language (H. Li \& Pitkänen, 2018). Language issues may become apparent when they read materials (C. Smith, 2020) and produce long pieces of academic writing, for example, dissertations (Qi \& Li, 2020). One of Li et al.'s (2018) participants said that he sometimes needed to look up unfamiliar words in his dictionary, which meant he failed to follow the lecture. In Sawir et al.'s (2012) study on the role of language proficiency in the security of students from non-English speaking countries in Australia, participants reported having problems with academic writing. Many needed to translate their work from their mother tongue into English, which potentially made their writing sound unnatural to native speakers. The authors argued that language proficiency is a key to students' active human agency. A lack of language fluency contributes to their limited confidence, which results in their behaviours being interpreted as passive.

International students may have difficulties communicating with instructors and classmates due to language issues, and as a result, they may prefer to sit together speaking their native language rather than interact with domestic students (C. Smith, 2020). They may find it hard to engage in group work (e.g., discussions or class presentations) which involves written and verbal communication skills (H. Li, 2017; C. Smith, 2020). Language issues involving 
comprehension problems are multifaceted. Terminology and subject-specific technical language may present difficulty and anxiety to international students (Cowley \& HyamsSsekasi, 2018). Low language proficiency affects their academic achievement (Andrade, 2006; Andrade \& Hartshorn, 2019; Chien, 2020) and understanding of teachers' speech (H. Li \& Pitkänen, 2018). Students' improvement in language ability helps them understand course content (H. Li, 2017).

In the everyday setting, proficiency in the language of the host country facilitates international students' sociocultural adaptation and interaction with local students (Alsahafi \& Shin, 2017; B. Yu, Bodycott, \& Mak, 2019). Better language skills are associated with a higher level of life satisfaction (Deloitte, 2008). In discussing the work experience of international students in Australia, Nyland et al. (2009) linked student-workers' language deficiency to their vulnerability. Sawir et al. (2012) identified challenges coming from language barriers, including isolation from local people, poor social interaction and adjustment, inability to integrate socially with the host community, stereotypes of locals, higher cultural stress, and lower level of life satisfaction. Language difficulties may involve both international and local students. Although having few problems in everyday communication, the Indian students in Kukatlapalli et al. (2020) had difficulties with the local New Zealand accent. Similarly, locals had difficulty with the Indian accent.

In summary, language has an important role in international students' experiences, including their learning and everyday experiences. In the academic setting, language issues may affect students' academic performance. In the everyday setting, language difficulties are likely to affect students' communication with people from different linguistic backgrounds. Overall, students' adjustment to the host environment may be affected by their lack of competency in the native language.

\section{Psychological adjustment experiences}

While undertaking education in a foreign country may open new doors for students' lives and be exciting for them, it may pose risks to emotional health and well-being. Psychological adaptation is an aspect of international students' experiences (Berry, 2017) and "refers to how comfortable and happy a person feels with respect to being into [sic] the new culture, 
or anxious and out of place" (Demes \& Geeraert, 2014, p. 91). While sociocultural adaptation is associated with "doing well", psychological adaptation is about "feeling well". The psychological adaptation of international students has been studied in relation to a range of health and well-being factors, many of which have been linked to negative symptoms and unpleasant emotions, for example, loneliness, homesickness, isolation, stress, depression, anxiety and low life satisfaction (Alloh, Tait, \& Taylor, 2018; Cowley \& Hyams-Ssekasi, 2018; J. Zhang \& Goodson, 2011) and pleasant feelings, for example, feeling exciting and inspiring (Chien, 2020).

International students are likely to experience significant emotional distress as a result of sojourning away from their families, friends, and familiar environments (Pacheco, 2020). Studies involving Vietnamese students in Australia by A. Le (2014) and L. T. Tran (2011) revealed that loneliness, isolation, and homesickness were common problems. In her review, Yan (2020) linked international students' acculturative stress with support and posited that those who experience high levels of acculturative stress and perceive low coping resources are more prone to anxiety, stress, and depression. Further, social support can relieve stress, improve health outcomes, and moderate the impacts of stress on mental health (C. Smith, 2020).

In addition to familial and friend-related issues, academic issues may contribute to international students' unpleasant emotions (Qi \& Li, 2020). Maintaining desired academic results may make students feel stressed (K. Yan, 2017). C. Wang et al. (2017), in a study on the learning experiences of Chinese nursing students in Australia, found that the students experienced fear and anxiety caused by unfamiliar education methods and assessment expectations. Li et al. (2018), exploring academic experiences of East Asian students at a U.S. university, revealed that nearly all of the participants had negative feelings (e.g., frustration, stress, exhaustion, and powerlessness) sometimes in their studies. Similarly, exploring the health experience of Nigerian students in the UK, Alloh et al. (2018) concluded that meeting academic demands, dealing with issues in an unfamiliar environment, and leaving home amplified the level of stress, which might lead to depression. Alloh et al. suggested that their female participants appeared to be more sensitive to stress and their symptoms were 
manifested in physical health more frequently than their male counterparts. They called for more support for female students.

Homesickness, which is defined as the distress triggered by being away from home (Thurber \& Walton, 2012), is prevalent amongst international students. This emotional experience is characterised by a feeling of loneliness, missing friends, family, and familiar places (Stroebe, Van Vliet, Hewstone, \& Willis, 2002). Discrimination may lead to homesickness which can affect students' wellbeing and academic work (Duru \& Poyrazli, 2011; Vergara, Smith, \& Keele, 2010). Students can be distracted from their studies by these negative feelings (Cowley \& Hyams-Ssekasi, 2018). Those who come from collectivistic cultures are prone to feeling homesick because they may be ill-prepared for independent living and may lack the necessary skills to live in a country with a dominant individualistic culture (Khawaja \& Stallman, 2011).

Weiss (1973) identified two forms of loneliness - personal and social loneliness. Personal loneliness is associated with the loss of contact with family members and characterised by anxiety and apprehension. Social loneliness is potentially caused by the absence of familiar social networks and may generate boredom and a sense of being excluded or rejected social loneliness. A sense of not belonging may lead to a range of negative feelings, such as anxiety, jealousy, depression, and loneliness (Osterman, 2001). Exploring the experiences of 200 international students in Australia, Sawir and colleagues (2008) found that many of them experienced a third form of loneliness - cultural loneliness which was created by the absence of familiar cultural and linguistic environments. Cultural loneliness may even impact students with sufficient personal and social support. Same-culture networks and strong relationships between international and local students may be useful for students in dealing with this form of loneliness.

While all students may feel homesick and lonely, international students may have greater levels of loneliness than local students (Neto, 2021). Many international students are from cultural backgrounds where strong family ties and friendship networks are emphasised (Marginson et al., 2010). The values and norms in their home countries shape their experience in host countries, especially where competition and individual achievement are 
the norms, and where extended family and tradition are not usually of similar importance, (Marginson et al., 2010). Such cultural differences may cause loneliness and isolation (Marginson et al., 2010; C. Smith, 2020).

Homesickness and loneliness may impact on several aspects of international students' experiences. Homesickness is sometimes associated with students' poor academic performance, social and emotional experiences (Alsahafi \& Shin, 2017; Thomas, 2020). Poyrazli et al. (2001), in their study of the adjustment experiences of Turkish international students in the USA, found that there is a negative correlation between the level of loneliness and academic performance. Similarly, Brennan (1982) and Dobson et al. (1987) suggest that loneliness may be associated with a loss of motivation and academic attrition.

Having a partner or family can make a difference to international students' experiences, especially postgraduate students (Loveridge, Doyle, \& Faamanatu-Eteuati, 2018). Being away from their partners or children often leads to students' homesickness, emotional turmoil, psychological stress, and negativity, which may affect their studies (Harvey, Robinson, \& Welch, 2017; Loveridge et al., 2018). In this respect, international students may face more severe problems than many domestic students who can visit home more regularly (C. Smith, 2020). Students who have a family may experience greater challenges than those who do not. If students' families move to the new country with them, these family members may face adjustment challenges and the students may need to support them, or the students may have difficulties in fulfilling the demands of family life while having to focus on their studies at the same time (Loveridge et al., 2018).

\section{Students' strategies in dealing with challenges and help-seeking behaviours}

Coping can be described as individuals' efforts to diminish emotional, physical, and psychological troubles (Tuncay, Musabak, Gok, \& Kutlu, 2008). J. Li et al. (2018) identified three strategies that their 13 East Asian student participants employed to deal with academic challenges - self-reliance (depending on self), dependence (relying on others for help), and isolation (depending on neither self nor others). Among these, self-reliance was the most common, followed by dependence which was used by eight participants. 
Strategies to deal with distress can be conceptualised in different ways and there may be overlaps among them. Lazarus and Folkman (1984) and Folkman (2008) proposed three types of coping strategies: problem-focused, emotion-focused and meaning-focused coping. A problem-focused strategy involves behavioural actions (e.g., taking measures to control stress stimulation factors) to overcome problems. An emotion-focused strategy refers to the use of behavioural and cognitive strategies (e.g., seeking emotional support from friends and family, or ignoring problems) to manage distress. Using meaning-focused coping, people draw on their beliefs or values to tackle problems. In their review, Smith and Khawaja (2011) found that positive coping strategies or adaptive coping strategies may be related to positive interpretation and acceptance while maladaptive coping strategies may involve denial, worry and self-blame.

A positive strategy for coping may be consciously taking a self-help approach. In a study on self-help coping strategies used by international students in Malaysia, Saravanan, Mohamad, and Alias (2019) found that students' strategies included sharing their problems with others, keeping themselves busy, engaging in physical activities, dealing with issues courageously, thinking positively, and relying on religious texts.

In their study on the coping strategies of East Asian students in the USA, Park, Lee, Choi, and Zepernick (2017) found that their nine graduate student participants utilised a range of strategies to resolve problems. They actively made efforts to deal with problems in coursework and academic stress. They also sought support from their American peers and co-nationals. Their American friends helped them with questions about local social systems and norms while their co-national friends provided emotional support. To deal with financial issues, they received financial support from their families. Their academic advisors helped them with academic, personal, and emotional matters. Some relied on their communities for emotional support. Campus resources (recreation centre and international students service centre) were used in the process of adjustment and managing stress. Besides such problem-solving strategies, avoidant coping strategies were employed. For example, one student chose to ignore experiences of discrimination. None of the participants sought assistance from counselling services to deal with their psychological problems. 
Exploring coping strategies of Mainland Chinese students in Hong Kong, Bhowmik, Cheung, and Hue (2018) found that the students utilised both adaptive and maladaptive coping strategies to tackle stress. Their adaptive coping strategies included attempting to improve their language skills, making friends with local students, watching local television, keeping in contact with relatives in the host country, attending Mainland Chinese students' association activities, seeking advice from Mainland Chinese peers with difficulties, not viewing cultural differences as contradictory, respecting cultural differences and adopting local cultural values. The students' maladaptive coping strategies were related to avoidance coping and emotion suppression.

Support services, which are based on or off campus, are important to international students' experiences (Ammigan, 2019). However, many factors contribute to students' underutilisation of support services for psychological issues. These include difficulties in understanding health services information, high costs, attitudes towards seeking professional assistance or health services (J. Li, Marbley, Bradley, \& Lan, 2016; Tang, Gui, Chen, \& Magueramane, 2018), not understanding their own health problems, doubts and discomfort about using services, and unawareness about the availability of services (N. Kim, Oh, \& Mumbauer, 2019). A student struggling with the language may find it difficult to describe an illness or symptoms in a foreign language (Forbes-Mewett \& Sawyer, 2016; Tang et al., 2018). Other factors, such as students' lack of perceived need for counselling and fear of stigma or judgement attached to seeking counselling, hinder them from seeking help from professionals (N. Kim et al., 2019; K. Yan, 2017). Instead, some students rely on their families and friends to deal with those issues (N. Kim et al., 2019; Tang et al., 2018). Students may feel more comfortable seeking assistance from university support centres with academic and career-related matters as this is more socially acceptable to them (Lértora, Sullivan, \& Croffie, 2017).

\section{Previous Studies on Vietnamese International Students' Experiences}

In this section, I critically review the literature on the experiences of Vietnamese international students and identify the research gap in this pool of literature (see Appendix 
F for a summary of studies). I then present how my study can address this gap and make an original contribution to the field.

Collectively, these studies have looked at various aspects of Vietnamese students' experiences (from psychological, academic, to socio-cultural) in different host countries from multiple perspectives. These include motivations for studying overseas, challenges in the everyday and academic settings, self-development, and readjustment experiences.

These studies identified various factors affecting students' decisions for studying overseas. The most common reason was to obtain better education (Miller, 2012; L. N. Tran, 2011). Many students were motivated by employment opportunities, job prospect improvement, and wealth betterment (M. Hoang, Moslehpour, \& Seitz, 2019; A. Le, 2014; Miller, 2012; Lien Pham, 2013). Other reasons were related to parents' and community's expectations, students' desire to gain qualifications from an English speaking country (M. Hoang et al., 2019; Lien Pham, 2013), self-development such as broadening knowledge, learning about new cultures and people, (A. Le, 2014; L. N. Tran, 2011), and personal growth (A. Le, 2014; Miller, 2012; L. N. Tran, 2011). Tuition fees and living costs were considered when choosing a university (M. Hoang et al., 2019). To a large extent, these motivations are similar to those of the wider international student population (Mazzarol \& Soutar, 2002).

Vietnamese students may encounter a range of difficulties in new environments. The most common issue was related to language proficiency (Miller, 2012; L. N. Tran, 2011; Vu, 2013; Vu \& Doyle, 2014; Wearing, Le, Wilson, \& Arambewela, 2015) both inside and outside the classroom (M. N. Nguyen \& Robertson, 2020). In class, students may have difficulties taking notes, understanding lectures, answering questions (Do, 2007), interacting with instructors, advisors, and local students (Lem, 2019; T. T. T. Nguyen, 2019; Wearing et al., 2015). Other issues may be related to unfamiliar teaching and learning approaches (Lem, 2019; Q. Nguyen \& Buckingham, 2019; Vu \& Doyle, 2014), limited understanding of plagiarism (T. Doan, 2012), and differences between Vietnamese and English writing conventions (Phan, 2001). In everyday settings, difficulties may include loneliness/ isolation, homesickness (A. Le, 2014; Lem, 2019; L. N. Tran, 2011), racism and alienation (Lem, 2019; L. N. Tran, 2011), 
unfamiliar diet (Lem, 2019), culture shock (A. Le, 2014; Wearing et al., 2015), mental distress (N. C. Pham \& Shi, 2020), and limited activities for international students (Lem, 2019).

Students had their own strategies to deal with challenges in the new and unfamiliar environments. In the USA context, students sought support from the local Vietnamese community and local American families (A. Le, 2014) or relied on themselves (Do, 2007) for personal issues. Students in Tran's (2011) study often participated in social activities to improve their experiences. Most participants considered self-reliance as the most beneficial (L. N. Tran, 2011). They might also seek emotional, practical and professional support from their networks (e.g., extended family members, colleagues, friends, and teachers) in Vietnam (M. N. Nguyen \& Robertson, 2020).

Studying overseas offered students positive transformative aspects. It provided them with opportunities and environment for improving English (Wearing et al., 2015) and sometimes led to changes in their social and academic identities (T. H. L. Bui, 2017). Vu and Doyle (2014) and L. Pham and Saltmarsh (2013) found that their participants became more independent and took greater responsibility for their learning. They valued the opportunities to gain new experiences and develop their academic, social, and professional skills. The student returnees in L. Pham's (2017; 2016) studies highlighted the importance of the knowledge and working attitudes that they acquired through international education for their anticipated career practices. Intra- and extra-curricular activities enabled them to improve their professional skills and career prospects. Starks and Nicholas (2017) were interested in how returnees embrace and contest aspects of multiple worlds and the complex nature of post-return identity after overseas study in Australia. The students adopted practices that they perceived as western in terms of body language, dress, movement, work practices, punctuality, queuing in public, queuing in class, and styles of speaking.

In recent years, there has been a shift within the international student experience towards an interest in how students enact agency and construct/reconstruct identities. Selfdevelopment has been identified as a motivation for overseas study (L. N. Tran, 2011). L. Pham and Saltmarsh (2013) found that the students in their Australian study identified with their Vietnamese identity and recognised their unique cultural traits in the new cultural 
environment. They could acquire and embrace aspects of Australian attributes to achieve their academic goals while going through complex processes of negotiating their networks, self-reflections, self-orientations and self-analysis of their values. Similarly, other researchers highlight students' self-formation, reflexive capability, and self-construction ( $T$. A. Bui, 2021; B. T. T. Nguyen \& Pennycook, 2018), emphasising that students may engage in a dynamic and on-going process of identity formation and re-formation (L. Nguyen, 2018). They may demonstrate agency when being strategic in dealing with challenges, transforming into a more confident self, and mobilizing their networks to deal with issues (M. N. Nguyen \& Robertson, 2020).

A number of studies have focused on the challenging re-adjustment process of Vietnamese returnees, suggesting that some students may find it hard to adjust to lives in Vietnam after overseas studies. A. T. Le and LaCost (2017) found that after returning from the USA, their participants encountered a range of issues, including loss of career opportunities, and unexpected issues with their familial and romantic relationships. They encountered reverse culture shock and had to make significant efforts to fit back into the local community and culture. In Pham's (2017) study on the experience of student returnees' re-engagement with workplaces and communities in Vietnam, the students were conscious of reverse culture shocks, such as lack of connections with local business, and limited local work experience and understanding of local society. Despite such awareness, they were poorly prepared for such challenges.

Few Aotearoa-New Zealand studies have explored the experiences of Vietnamese students. There are four studies - two published and two unpublished master's theses, with a focus on Vietnamese students. For his master's thesis, Dao (2005) investigated how Vietnamese undergraduate and postgraduate students used a library and its services. Focusing on undergraduate students, $\mathrm{Vu}$ (2013) used narrative inquiry for her master's research to explore their experiences at a New Zealand university. Vu and Doyle (2014) examined how undergraduate twinning students from Vietnam positioned and repositioned themselves and their teachers. More recently, Q. Nguyen and Buckingham (2019) investigated master's students' approach to using sources in assignments, focusing on stages of engagement (e.g., understanding source-use expectations, identifying appropriate sources, and incorporating 
content from source texts into assignments). These four studies appear to be the total of New Zealand-based research on Vietnamese students. This suggests a dearth of research on Vietnamese students' experiences, especially at postgraduate levels where students are likely to have different social and academic experiences from undergraduate students. Besides, most studies on Vietnamese international students have been survey-based (Brisset et al., 2010; H.-H. Pham, Lai, \& Vuong, 2019), standard case study (M. N. Nguyen \& Robertson, 2020; Q. Nguyen \& Buckingham, 2019), or employed narrative inquiry (Dang \& Tran, 2017; Vu \& Doyle, 2014), with an exception of a study by Nguyen (2018) who utilised a qualitative case study located within a broader phenomenological approach. No studies have explored students' lived experiences using interpretative phenomenological analysis (IPA).

Briefly, while there is considerable English language literature on Vietnamese international students' experiences in Anglophone settings, especially in Australia, few studies have focused on Vietnamese postgraduate students in Aotearoa-New Zealand. No studies have specifically explored students' everyday living experiences. Only one study employed phenomenology within the case study to investigate students' experiences. These suggest a dearth in the literature and indicate the value of further investigation of Vietnamese postgraduate students' lived experiences in Aotearoa-New Zealand, employing IPA to explore the individual experiences of Vietnamese students.

\section{Chapter Summary}

For many students who are undertaking higher education overseas, entering an unfamiliar environment may be challenging. They may experience discomfort and distress from various sources. In the academic setting, they may have difficulties with the new and unfamiliar teaching and learning styles, heavy workload, and writing conventions (e.g., issues related to referencing and plagiarism). In everyday living, issues may be related to their inability to build meaningful connections with others, loss of familiar networks, financial burden, and living arrangement issues (e.g., housing costs, poor housing conditions, and relationships with flatmates). Multiple factors contribute to students' experiences. Language difficulties may have an impact on their experiences both within and outside universities. With limited 
English proficiency, they may find it hard to communicate with others or express their ideas in written assignments.

A review of the literature on Vietnamese international students' experiences identified a prevalence of survey-based studies, case studies, or narrative inquiry. Some studies have failed to recognise students as agentic beings. While students' living arrangements play a critical role in their lives and tend to significantly impact on their studies, no previous research has investigated issues related to Vietnamese students' housing experiences. This lack of research suggests a need for research on Vietnamese students' experiences in everyday settings. Addressing this research gap, my study is likely to make a valuable and original contribution to the field. In the next chapter, I present the research methodology and design of this study, and the rationale for choosing the research approach. 


\section{Chapter 4 | Research Methodology and Design}

In this chapter, the methodological approach and the design of the study are presented, and I show how the research paradigm and the underpinning ontological, epistemological, axiological, and methodological assumptions are linked to the research aim. After introducing phenomenology as a mode of inquiry and types of phenomenology, I outline differences between IPA and other qualitative approaches (e.g., narrative inquiry and case study) and give reasons for my choice of IPA. The research design is detailed, including participant recruitment, data collection, and data analysis. I then describe the ethical issues considered in the study. Finally, I set out how the trustworthiness of the study was ensured.

\section{Aim of the Study}

My study explored the experiences of Vietnamese students in everyday and academic settings. The research question was: "What are Vietnamese students' lived experiences of everyday life and study in Aotearoa-New Zealand whilst they complete a master's degree?" A qualitative methodology was a good fit for this study which aimed at acquiring an in-depth understanding of the processes through which the students make sense of their personal, social, and academic experiences, and for looking at their self-reflection and account of the processes. An interpretative phenomenological approach was chosen because it allowed me to explore in detail how participants understood their personal and social world and what particular experiences meant for them, and also to capture the essential meaning of their experiences (J. A. Smith \& Osborn, 2008).

\section{Research Paradigms}

Worldviews (Creswell, 2014), also known as research paradigms (Lincoln, Lynham, \& Guba, 2018; Mertens, 2014), can be defined as "a basic set of beliefs that guide action" (Guba, 1990, p. 17). Creswell (2014) identified four main worldviews that researchers bring to inquiry: post-positivism, constructivism, transformative, and pragmatism. Constructivism includes the interpretivist worldview and was utilised for this study. Interpretivism is 
characterised by understanding, multiple participant meanings, social and historical construction, and theory generation. It emphasises that people attempt to understand the world in which they live and work. As suggested by Creswell (2014), I consider that people construct different subjective meanings or accounts of their experiences, and researchers will also make sense of these meanings.

A researcher's philosophy and view of knowledge and the world shapes what they are interested in and how they seek to understand a phenomenon. Within Western social science, a common way of discussing researchers' philosophies has been to consider these in relation to four types of assumptions: methodological assumptions (i.e., beliefs about the process of research), ontological assumptions (i.e., beliefs about the nature of reality), epistemological assumptions (i.e., beliefs about how we know what we know), and axiological assumptions (i.e., beliefs about ethics, aesthetics, religion, and values) (Creswell \& Poth, 2017; Lincoln et al., 2018).

There are a number of reasons for the important role of research philosophies. In addition to the reasons related to training that researchers received, the scholarly community where they work, and the evaluation of reviewers, philosophies enable researchers to understand and reflect on problems, formulating research questions and guiding researchers to find answers to the questions (Huff, 2009).

Researchers working within a qualitative methodology assumption, which focuses on the procedures of research, are typically studying a topic within its context (Creswell \& Poth, 2017). Qualitative research is associated with inductive reasoning or a bottom-up approach (i.e., researchers start with perspectives of participants, specific observations, or real examples of a phenomenon, then progress to broader generalisations or generating theories). A qualitative study may have an emerging design (i.e., a design which evolves from description to themes to broad generalisations).

Philosophical assumptions underpinning my study are based on social constructivism. I accept the ontological assumption, the nature of reality (Creswell \& Poth, 2017), related to constructivism and believe that the participants have different experiences, and interpret or understand their experiences in various ways (Creswell \& Poth, 2017). I utilised IPA to 
capture the essence of the Vietnamese postgraduate students' experiences in academic and everyday settings. When reporting the findings, I attempt to capture multiple aspects of their experiences by providing different themes and sub-themes along with their actual words. However, I acknowledge that the interpretation of data was in part influenced by my experiences as a Vietnamese postgraduate student in Aotearoa-New Zealand, and in part by my knowledge of how other researchers have interpreted and theorised international students' experiences.

With a constructivist worldview, knowledge and understanding are seen as constructed through and by social interaction (Creswell, 2014). In this respect, researchers and their participants engage to create knowledge and understanding. I believe that participants have various and subjective understandings of their experiences. Social constructivism is related to interpretivism (Creswell, 2014; Creswell \& Poth, 2017; Denzin \& Lincoln, 2011). In interpretivism, researchers seek to make sense of the meanings that their participants have about their experiences and are interested in capturing patterns of the meanings. In this process, researchers' experiences and background affect their interpretation of data. Taking a perspective of interpretivism, I am aware of this complexity of students' perspectives and try to capture it. Interview questions were open-ended so that students could share their perspectives on their experiences. I focused on the settings in which the students lived and studied, which enabled me to understand how they were related to their experiences.

Epistemology is a term that relates to theories of knowledge and includes questions like "How do we know what we know?" (Creswell, 2014, p. 23), or 'What counts as knowledge?' (Creswell \& Poth, 2017, p. 54). It is a central consideration for researchers because academic inquiry aims to produce new knowledge (Kovach, 2017). I take the approach that knowledge can be acquired by investigating participants' accounts of their subjective experiences (Creswell, 2014). In this regard, shared language and cultural understandings between me as a researcher and the participants played an important role in helping me establish a good relationship with them so that they were comfortable about discussing various aspects of their experiences. The interviews were conducted in Vietnamese so that they could understand and answer the questions more fully. 
The axiological assumption underpinning my study is about the role of values (Creswell \& Poth, 2017). In qualitative studies, researchers typically agree that everyone has their own values. This assumption is characterised by a focus on researchers' values in their studies (Creswell, 2014). Values are associated with ethics. Because my study is interpretative in nature, it is important that I position myself as a researcher and consider my own biases and preconceptions that could potentially influence my interpretation of data. To this end, my interpretation of the interviews was partly shaped by my own experiences, values and background as a Vietnamese postgraduate student in Aotearoa-New Zealand, but I acknowledge that the participants made sense of their experiences differently. This was partly because of cultural values that they held. In reporting the findings, I highlight the unique story of each individual. I was respectful of the participants and their stories. When interacting with them and interpreting the data, I was cautious about words or behaviours that could be viewed as being judgemental of them. Ethical issues related to my study will be considered later in this chapter.

\section{Methodology}

I was interested in examining various aspects of Vietnamese international students' experiences and capturing the essence of these experiences. In this section, I set out the thinking behind my choice of the research methodology. As part of this, I describe the roots and connections between phenomenology as a philosophy and phenomenology as a research methodology, highlighting some of the core concepts that underpin phenomenology. I will discuss IPA in terms of its strengths and weaknesses, and why I chose IPA for my study rather than other forms of phenomenology, namely, descriptive phenomenology and hermeneutic phenomenology.

\section{Phenomenology}

In this section, I outline the concept of phenomenology and present the philosophical underpinnings of phenomenology. These ideas are critical to the concept of IPA that I present in a later section. 


\section{What is Phenomenology?}

Phenomenology is both a school of philosophy and a family of qualitative research methods. The term 'phenomenon' is derived from the Greek meaning to flare up or to show itself (Moustakas, 1994), and the term 'phenomenology' (Greek words: phainomenon and logos) to bring into the light (Pringle, Hendry, \& McLafferty, 2011). Phenomenologists claim that an understanding of a phenomenon can be gained through interactions between researchers and people who experience the phenomenon. This leads to the subjective, inductive, and dynamic natures of phenomenological studies (Polit \& Beck, 2005).

Phenomenology has its roots in the work of four major thinkers: Husserl, Heidegger, MerleuPonty, and Gadamer (Dowling, 2007). Husserl (1859-1938) began this philosophical movement which was then developed by his student Heidegger and other phenomenologists (Langdridge, 2007). Whilst Husserl, Heidegger and Merleau-Ponty did not develop phenomenology as a research approach, their work is used to support contemporary qualitative research (Fleming, Gaidys, \& Robb, 2003).

Descriptive and interpretive phenomenology is based on the work of Husserl (1973) and Heidegger (1988) respectively and these are the major stands of phenomenology in philosophy which have influenced the development of phenomenological research methods. As a research method, phenomenology includes several phenomenological research approaches and schools. Gill (2014) outlines five major typologies of phenomenological methodologies belonging to either Husserlian descriptive phenomenology, including Sanders' (1982) phenomenology, Giorgi's (2010) descriptive phenomenological method and van Manen's (2007) hermeneutic phenomenology, or Heideggerian interpretive phenomenology, including Benner's (1994) interpretive phenomenology and Smith's (2008) interpretative phenomenological analysis. Cohen and Omery (1994) list three schools of phenomenology (Edetic or descriptive phenomenology, hermeneutics and the Dutch school of phenomenology). Despite such divergence, there are some fundamental similarities amongst the phenomenological approaches: drawing on phenomenological philosophy, focusing on the meanings of people's experiences, 
considering experiences from experiencers' perspectives, using homogenous samples, and searching for themes from data (Gill, 2014).

\section{The philosophical foundations of phenomenology}

In the following section, I briefly present a distinction between the philosophical perspectives of Husserl and Heidegger. I highlight some fundamental concepts that underpin the two perspectives. This section provides a basis for my choice of IPA rather than descriptive phenomenology or other qualitative research approaches, which will be presented in a later section.

\section{Husserl (1859-1938)}

As a philosophical movement, phenomenology emerged in Germany at the beginning of the $20^{\text {th }}$ century (Matua \& Van Der Wal, 2015). Husserl developed phenomenology around the central concept of intentionality (Moran, 2000). Intentionality is associated with consciousness and has a different meaning in philosophical phenomenology than the everyday meaning. This concept indicates that whenever people "are conscious [...], it is always to be conscious [...] of something" (Langdridge, 2007, p. 13). Husserl asserted that "there is a phenomenon only when there is a subject who experiences the phenomenon" (Sadala \& Adorno, 2002, p. 282). On this basis, intentionality refers to the separate but linked relationship between an individual's mind (or consciousness) and the world (Langdridge, 2007). For Husserl, experience is an important source of knowledge, and phenomenology is the study of human consciousness and experience. The aim of Husserl's phenomenology is to "see things 'as they are' through intuitive seeing" (Laverty, 2003, p. 23), or study people's experiences of phenomena from an unbiased perspective (Valle, King, \& Halling, 1989).

Husserl's work provided a foundation for descriptive phenomenological research methodologies which aim to describe the essence of people's experiences (Gill, 2014). Husserl's phenomenological inquiry focuses on individuals' conscious experiences. The concept of phenomenological reduction was proposed by Husserl to enable the essence of phenomena to emerge and is central to phenomenology's epistemological strategy (F. E. Racher \& Robinson, 2003). In his descriptive method, 'reduction' means that an individual 
"reduces the world as it is considered in the natural attitude to a world of pure phenomena" (Valle et al., 1989, p. 11). This requires phenomenologists to look at a phenomenon in an unprejudiced way so that the phenomenon can be interpreted and described properly (Dowling, 2007).

'Reduction' is part of the analytical framework of many methodologies in phenomenology (Gill, 2014). Transcendental or phenomenological reduction is one type of reduction which is associated with bracketing or epoché - a Greek word meaning to stay away from the everyday way of perceiving things (Moustakas, 1994). In this approach, phenomenologists set aside their personal knowledge and assumptions to explore and describe a phenomenon as experienced by the person whose experience it is, which is believed to enable people's experiences of such phenomena to be seen in a neutral and transcendental way (Giorgi, 2009). However, the question of whether people can completely bracket off their preconceptions seems to be an ongoing debate amongst transcendental phenomenologists (those who base their work on Husserl) and existential phenomenologists (those who draw on the work of Heidegger, Sartre, and Merleau-Ponty) (Langdridge, 2007).

Besides 'phenomenological reduction', Husserl introduced some of the key concepts associated with phenomenology today, such as life world, lived experience, and essence. Husserl (1900) conceptualises the "life world" as what people experience without going through the interpretation process. Lived experience is related to individuals' immediate consciousness of life (Dilthey, 1985). For phenomenological research, essence, which is essential aspects or qualities (or universal structures) of things, is important because it makes things what they are, and without it things could not be what they are (Max van Manen, 1990). In focusing on the essence, phenomenologists move beyond the description of people's experiences and aim to consider the underlying structure of those experiences. Husserl suggested that through analysing and describing each individual's experience of a phenomenon, phenomenologists can identify its structure. Such structures include the essence of the experience and its individual meaning (Langdridge, 2007).

Descriptive phenomenological methodologies aim to search for essence (Gill, 2014). Husserl emphasised the role of imaginative free variation in enabling phenomenologists to capture 
the essence of people's experiences. Phenomenologists seek to approach the phenomenon under study from various points of view, thinking of different variations of the phenomenon under investigation, and attempting to see what the essential aspects of that phenomenon are, or what makes the phenomenon to be what it is. Husserl believed that phenomenology "must bring to pure expression, must describe in terms of their essential and invariant concepts, the essences which directly make themselves known in intuition" (Husserl, 2001, p. 86). According to Husserl, essence is important in all kinds of knowledge, and his focus on describing essence has become an important part of phenomenological methodologies that draw on his work (Gill, 2014).

\section{Heidegger (1889-1976)}

Husserl's student, Martin Heidegger, was another leading figure in phenomenology who attempted to establish a more interpretive approach to phenomenology by connecting personal experience to our 'relationships to the world and others' (J. A. Smith et al., 2009, p. 21). Both Heidegger's and Husserl's phenomenology focus on people's lived experiences and the essence of experiences (Dowling, 2007). However, there are fundamental differences between these two forms of phenomenology. Whilst Heidegger and Husserl share a focus on the essence of experiences, Heidegger believes that, in phenomenology, interpretation should be given more emphasis than the mere description (F. Racher, 2003). He recognises the role of hermeneutics underpinned by the ontological perspective in the interpretation process (F. E. Racher \& Robinson, 2003). In other words, Heidegger has a different view on how phenomenologists can gain an understanding of the essential aspects of people's experiences.

In contrast to Husserl's phenomenology, Heidegger's (1996) phenomenology has a different subject and method. His work led to the emergence of hermeneutic phenomenology (or hermeneutic-existential phenomenology), within phenomenological philosophy, which focused on understanding the world as it is lived (lived experience) and interpretation of the meaning of experiences. Unlike Husserl's phenomenology with an epistemological commitment, Heidegger's phenomenology is ontological, and he regards lived experience as an interpretive process (F. E. Racher \& Robinson, 2003). He has a different way of 
exploring the lived experience utilising hermeneutics as a research method. He proposes the concept of the 'hermeneutic circle', which is one between pre-understanding and understanding.

A characteristic of the phenomenological research methodologies that use Heidegger's work as a foundation is their commitment to interpretation and hermeneutics. Heidegger believes that how people made sense of their experiences of a phenomenon was determined by such factors as culture and society, and people could not be completely independent of these factors. He argues that an "interpretation is never a presuppositionless apprehending of something to us" (Heidegger, 1996, p. 141). On this basis, interpretive phenomenologists are not expected to set aside their personal assumptions and experiences and need to interpret people's experiences in relation to the environment in which those people exist. In this way, Heidegger's interpretive approach to exploring people's experiences challenges Husserl's use of epoché/ bracketing as a way to search for the essence of human experiences.

\section{Interpretative Phenomenological Analysis (IPA)}

In this section, I introduce the concept of IPA. This is followed by a critique of this research approach.

\section{What is IPA?}

IPA is a relatively new approach to qualitative research on experience (J. A. Smith \& Osborn, 2008). It was developed as an approach to qualitative research in the UK in the 1990s by Jonathan A. Smith (J. A. Smith, 1994, 1996). Initially it was used mainly by psychological researchers (Eatough \& Smith, 2017). However, IPA has gained in popularity in other fields, including education, and has become one of the most widely used phenomenological approaches (Dowling \& Cooney, 2012).

As its name suggests, IPA "concurs with Heidegger that phenomenological inquiry is from the outset an interpretative process" (J. A. Smith et al., 2009, p. 32). This approach is phenomenological in that it allows researchers to gain a detailed understanding of participants' lived experiences of the phenomenon under investigation, looking at things 
(e.g., experiences, events) from participants' viewpoints (Pietkiewicz \& Smith, 2014). It is interpretative because it requires researchers to endeavour to interpret participants' experiences through a complex meaning-making process (Pietkiewicz \& Smith, 2014).

IPA focuses on aspects of experiences that matter to people (Eatough \& Smith, 2017). Within IPA, there is acknowledgement that people can give different accounts for their experiences of similar events or circumstances. These accounts are associated with participants' own thoughts, feelings, or judgements. The subjectivities of experience make it almost impracticable for researchers to have direct access to participants' personal and social worlds (Eatough \& Smith, 2017). In IPA, a researcher accepts that it is impossible to avoid biases, assumptions, and preoccupations, and acknowledges them when conducting a study. He/she will use them to gain understanding of participants' experiences of events, and reflect on how they potentially shape findings (Willig \& Stainton-Rogers, 2017).

IPA is concerned with the exploration of individual experience in a systematic way (Tomkins, 2017). It is underpinned by phenomenology, hermeneutics, and idiography. Smith et al. (2009) express IPA's strong commitment to phenomenology and hermeneutics, saying that 'without the phenomenology, there would be nothing to interpret; without the hermeneutics, the phenomenon would not be seen.' (p. 37). IPA is idiographic with the initial focus on understanding the individual experience.

Phenomenology can be regarded as the "study of human experience and the way in which things are perceived as they appear to consciousness" (Langdridge, 2007, p. 10). In phenomenology, researchers are interested in uncovering meanings, seeking to understand people's life world through examining their thoughts, memories, and feelings. Rooted in phenomenology, IPA attempts to understand the meanings that participants attach to their experiences of events and circumstances (J. A. Smith et al., 2009). Participants are treated as 'experiential experts' in the issue under investigation, and are encouraged to tell their stories from their viewpoints (Eatough \& Smith, 2017). IPA researchers refer to participants' context-dependent life worlds, for example, social and cultural characteristics (Eatough \& Smith, 2017). In this regard, IPA goes beyond simply describing a particular experience, it 
interprets what it means for a particular participant to have such experiences in a particular context (Noon, 2017).

Hermeneutics refers to the "practice of art of interpretation" (Dallmayr, 2009, p. 23) which is associated with "the restoration of meaning" (Ricoeur, 1970, p. 8). IPA is hermeneutic in that IPA researchers assume the central role in the data analysis and interpretation (Brocki \& Wearden, 2006). IPA's interpretative engagement is underpinned by the hermeneutic circle and IPA's 'double hermeneutic' (J. A. Smith \& Osborn, 2008). Smith et al. (2009) assert that the hermeneutic circle is about the interactive relationship between the parts and the whole, which is similar to the ones between a single word and a sentence. A person needs to understand words before being able to make sense of the whole sentence, and the understanding of a sentence informs the understanding of its words. With regards to double hermeneutic, IPA researchers are involved in a process where "the participant is trying to make sense of their personal and social world; they are trying to make sense of the participant trying to make sense of their personal and social world" (J. A. Smith, 2004, p. 40). Smith and Osborne's (2008) four key stages of inductive analysis are underpinned by double hermeneutic. On this basis, the quality of an IPA study is in part dependent on both participants' ability to give accounts of their experiences, and researchers' ability to interpret these accounts. Because, in IPA, researchers' prior knowledge plays an important role in the interpretation process, it is accepted that gaining an exact understanding of participants' world is almost impossible (Heidegger, 1996). On this basis, a more achievable objective is that IPA researchers attempt to get as close to participants' world as possible (Michael Larkin, Watts, \& Clifton, 2006).

IPA has an explicit idiographic focus, which means that it aims to gain an in-depth understanding of each participant's experiences before progressing to the next or more general claims (Eatough \& Smith, 2017). IPA's commitment to idiography makes it different from most of the other phenomenological methodologies (Gill, 2014). This nature of IPA requires researchers to examine intensively how a person makes sense of a particular experience in his/her unique context, which allows for an informative understanding of their experiences. The idiographic focus is demonstrated not only in the early stages which examine each single case, but also in subsequent steps which aim to find patterns across 
cases. Researchers need to be clear how the accounts of each participant fit in with more general themes (J. A. Smith \& Osborn, 2008). Besides, the use of single-person case studies and a small sample size, which aims to capture the richness of individual experiences, shows IPA' commitment to idiography (Eatough \& Smith, 2017).

\section{Critique of IPA}

Willig's (2013) criticism is that whilst IPA is able to produce rich descriptions of participants' experiences, it does not aim to explain the cause or origin of such experiences, which can limit researchers' understanding of phenomena. If they wish to have a good understanding of participants' experiences, they need to look at the conditions that allow the experiences to occur. However, it should be noted that IPA researchers are not interested in providing possible causal explanations for the experiences. Phenomenological inquiries, including IPA, aim to understand the meanings of people's experiences rather than predicting or explaining such experiences (J. A. Smith, 2004; Max van Manen, 1990).

IPA has been criticised for being ambiguous (Giorgi, 2010), predominantly descriptive, and not really as interpretative as its name suggests (Brocki \& Wearden, 2006; Hefferon \& GilRodriguez, 2011). However, many IPA researchers and thinkers have addressed this criticism by discussing and outlining the methodological and philosophical underpinnings of IPA.

Another limitation of IPA is that researchers cannot know whether they can accurately capture the meanings of their participants' experiences (Willig, 2013). Willig (2013) argues that whilst phenomenology as philosophy is associated with introspection, and philosophers can explore their own experiences through 'phenomenological meditation', phenomenology as a research method is strongly dependent on the accounts of participants. She raises questions about researchers' and participants' ability to successfully communicate the nuances of experiences. This is particularly true when participants are patients with communication difficulties. Tuffour (2017) considers such criticisms as elitist in the implication that participants need to be eloquent and argues that researchers needed to have strategies to enable rich data to be gathered. 
The use of smaller sample sizes in IPA than in other approaches, such as grounded theory, has exposed it to criticisms as to generalisability to the larger population (Malim, Birch, \& Wadeley, 1992). Responding to such criticism, J. A. Smith et al. (2009) assert that IPA researchers focus on "theoretical transferability rather than empirical generalizability" ( $p$. 51). They further argue that a small number of participants enables a detailed case-by-case analysis which may be difficult to have with larger samples. Wagstaff et al. (2014) add that a large sample size may generate too much data which potentially leads to an emphasis on common themes and loss of idiographic detail. In phenomenology, participants' rich qualitative accounts of experiences are far more important than the amount of data (Sanders, 1982).

\section{IPA or other kinds of phenomenology?}

Despite differences in focusses and assumptions, different forms of phenomenological approaches, including IPA, are "part of a stable of closely connected approaches which share a commitment to the exploration of personal lived experience" (J. A. Smith, 2004, p. 41). Whilst pure description is sometimes stated as a fundamental characteristic of phenomenological research (Max van Manen, 1990), "phenomenology without hermeneutics can become shallow" (Todres \& Wheeler, 2001). Max van Manen (1990) connects phenomenology and hermeneutics. Phenomenological researchers explore experiences as lived by individuals rather than separated from them (Max van Manen, 1990). For Max van Manen (1990), "A universal or essence can only be intuited through a study of the instances as they are encountered in lived experience" (p. 10). He describes how hermeneutic phenomenology enables researchers to understand people's experiences within the context of their life world, and shows that hermeneutic phenomenology can be applied to education, health, and nursing research (J. A. Smith et al., 2009).

Descriptive phenomenology and interpretative phenomenology have commonalities, for example, the Husserlian epistemological foundation (Matua \& Van Der Wal, 2015). Descriptive phenomenology was considered as a possible alternative for the current study. The participants' descriptions of what they encountered in their lives and studies would enable me to uncover their lived experiences. However, there are a number of 
methodological differences between the two approaches (Matua \& Van Der Wal, 2015). Descriptive phenomenology is used when a researcher is interested in describing the essence of the phenomenon itself (Idczak, 2007). IPA is employed when a researcher wants to learn about the meaning of the phenomenon for the participants and how they make sense of the situations they find themselves in (J. A. Smith et al., 2009). I wanted to interpret and describe the experiences of Vietnamese international students, seeking in-depth understanding of how they were making sense of their experiences, and what meanings a particular experience held for them. With this purpose in mind, I believed IPA was more suitable for my study.

Besides, there is an assumption that, in using descriptive phenomenology, researchers need to bracket their biases and personal experiences (Idczak, 2007). In IPA, they acknowledge their biases and prior knowledge are part of their interpretation and influence the data analysis (J. A. Smith et al., 2009). Although I appreciated the value of a bracketing process, I was concerned it would be impossible for me, as a researcher and a Vietnamese international student, to discard my prior experiences and preconceived notions of the phenomenon. I believed that my own experiences and sense-making might give depth and breadth to my analysis. On this basis, I decided to reject descriptive phenomenology and select IPA.

\section{Rationale for selecting IPA}

Researchers have developed and employed many qualitative approaches to study individuals' experiences in educational contexts. Generally, there are both differences and overlaps amongst them (J. A. Smith et al., 2009). Researchers may select a particular qualitative approach based on their research questions, given that each approach has a different perspective on what data are, what inference can be made from data, and what researchers may expect to gain through data analysis (J. A. Smith et al., 2009).

Personally, a qualitative design was selected because I love to write in a literary style. I wanted to tell the Vietnamese students' stories in a way that would show the diverse aspects of their experiences and enable readers to understand them from the participants' perspectives, and to see what it may be like to be a Vietnamese international student in 
Aotearoa-New Zealand. Having decided on employing a qualitative research approach, I considered what methods would be best to answer my research question. Some alternative methods were narrative inquiry, case study, and Grounded Theory.

Initially, narrative analysis or inquiry was one research method that I considered. There are both similarities and differences between narrative inquiry and IPA. Narrative inquiry focuses on how the stories are told, seeking to explore how the structure of individuals' stories may influence their experiences (Chase, 2000). However, like IPA, narrative inquiries focus on participants' accounts of their lived experiences (Chase, 2000). Narrative inquiries explore the experiences of an individual or a small group of participants, which causes such studies to be similar to IPA. A fundamental difference between the two approaches is their conceptualisation of data. Whilst narrative inquiry considers meaning as originating from words, and "takes as its object of investigation the story itself" (Riessman, 2002, p. 218), IPA uses verbal data to understand people's lived experiences (Chapman \& Smith, 2002), and is concerned with what is told by the participants, the meanings of experiences, and how they make sense of these experiences (J. A. Smith et al., 2009). On this basis, IPA is suitable for researchers who regard experiences per se as the object to explore rather than the stories of experiences.

Case study design was also considered; however, it was not suitable for my research questions. My purpose was not to discover the participants' experiences nor present them as cases. I was interested in IPA because it enabled me to understand and describe the essence of the participants' experiences. I wanted to explore the essential aspects of their experiences and what their experiences were like from their points of view.

Besides these considerations in relation to IPA and other research methods, IPA has some strengths that I found appealing. It explicitly recognises the central role researchers in analysing and interpreting participants' experiences (Willig, 2013). IPA researchers engage in "double hermeneutics", meaning that they interpret what their participants have interpreted (J. A. Smith et al., 2009). Researchers' conceptions and standpoint contribute to the interpretative analysis. This characteristic of IPA allows me to use my experiences as 
a Vietnamese international student and take advantage of my shared culture with the students to better see and understand their multifaceted experiences.

Whilst IPA is rooted in psychology, this approach has been shown to be a powerful tool for researchers in educational contexts (Noon, 2018). Individuals' subjective experiences may be what these researchers wish to understand, and IPA is particularly suitable for this purpose (Noon, 2018). IPA attempts to understand individuals' experiences from their own viewpoints and capture the meanings such experiences hold for them (Eatough \& Smith, 2017). Furthermore, the inductive feature of IPA may give rise to unexpected responses. J. A. Smith et al. (2009) asserted that "on the one hand they tell us something we did not even anticipate needing to know; on the other, because they arise unprompted, they may well be of particular importance to the participant" (p. 58).

As a novice researcher, I was attracted by IPA because it provides a specific and structured framework for conducting a study that not all qualitative research approaches have, for example, the ontological and epistemological underpinning a study (Michael Larkin et al., 2006), expected types of research questions, sampling strategies, and steps for data analysis (M Larkin \& Thompson, 2012; J. A. Smith et al., 2009).

Every qualitative research approach has its own advantages and disadvantages, and it is important for a researcher to be aware of these. Whilst I value the potential of other methods to allow for an understanding of Vietnamese international students' experiences, I decided on IPA for its suitability to my research purposes and its strengths in comparison to other qualitative research approaches.

\section{The link between the theoretical approach and IPA as a research approach}

IPA recognises the importance of social historical and cultural factors in individuals' experiences, and numerous factors in a host country. IPA researchers are interested in capturing the essential meaning of experiences in relation to such factors (J. A. Smith et al., 2009; J. A. Smith \& Osborn, 2008). This is consistent with Berry's framework which highlights the importance of factors in home and host countries in individuals' experiences, and with 
Maslow's theory which points to the critical role of having the need for shelter and safety met in everyday life.

Further to this, IPA is well suited to Marginson's concepts of international students' agency and self-formation. Smith et al. (2009) draw attention to the importance of researchers' engagement with participants' experiences relating to agency and suggest that individuals' experiences may be "the result of proactive agency on the part of the person" (p. 3). On this basis, I believe that IPA is compatible with Marginson's theory and allows me to capture the essence of students' transformation.

Finally, IPA focuses on how individuals make sense of their significant experiences (J. A. Smith et al., 2009), which may be positive or negative and may happen unexpectedly. In this study, I explored how Vietnamese students reflected on their experiences of living and studying in Aotearoa-New Zealand. Some students may have pleasant experiences (e.g., the availability of peer support or self-transformation). Others' experiences are less favourable (e.g., difficulties with language and cultural dissonance). This focus of IPA well matches with the theoretical frameworks used in this study.

\section{The Research Site}

The research site is VUW, which is a research-intensive university. Located in Wellington, the capital city of New Zealand, it offers undergraduate and postgraduate degrees and has over 22,000 students, 3,600 of whom are from outside the country. It has partnerships with universities and organisations around the world and provides a joint programme in premises at a partner university in Vietnam.

VUW is conscious of the critical role of students' experiences. The university offers a range of support and instruction for international students before, upon and post-arrival, for example, an international student orientation, and workshops. The assistance may relate to student visas, accommodation, preparation for travelling, arrival meeting service, and study support. Such services seek to help students adjust to lives and studies in Wellington. The university is a signatory to The Education (Pastoral Care of International Students) Code of Practice provided by the New Zealand Ministry of Education. 


\section{Participant Recruitment and Profiles}

In this section, I present the rationale for sample size and recruitment strategies. This is followed by a description of the participants' demographic information.

\section{Participant recruitment}

The study population was 10 Vietnamese international postgraduate students at VUW. This sample size is within the recommended range for IPA studies. A small sample is appropriate for an IPA study which seeks to produce a detailed examination of each particular case (Pietkiewicz \& Smith, 2014). A sample that is too large is more likely to be a problem for IPA researchers than a sample that is too small, and higher numbers do not necessarily lead to 'better' work (J. A. Smith et al., 2009). An IPA approach should focus on the depth rather than the breadth of the study (Pietkiewicz \& Smith, 2014). As an idiographic approach, IPA requires purposive sampling which involves choosing a relatively homogenous group of participants (J. A. Smith et al., 2009). This allows for detailed understanding of each participant's experiences of a phenomenon. Also, this sample size enabled me to keep the data manageable within the constraints of a doctoral project.

Purposive sampling was utilised (Creswell, 2014). The purpose of sampling in IPA is to select a specifically defined group relevant to the research questions (J. A. Smith \& Osborn, 2007). I was specifically interested in master's students. I had developed an interest in the experience of this student population when I was in Vietnam through discussion with my friends who were master's students in other countries. Another criterion was that students had been studying at VUW for at least six months in order for me to explore the diverse aspects of the students' lived experiences.

A number of strategies and channels were employed to recruit participants. Fliers were designed and posted on notice boards in VUW campuses (see Appendix D). Besides the flier, I prepared a brief description of my study. The flier and the description were posted on the Facebook page of the Vietnamese Students' Association in Wellington (VSAW) and emailed to postgraduate students in my network in various faculties of VUW. 
A Vietnamese festival - V-District 2017 - which attracted many Vietnamese students, happened to be organised at the Wellington Night Market at the time I was recruiting participants. I went to the market and advertised my study to Vietnamese students I came across. I also gained access to potential research participants through the snowballing technique (Johnson \& Christensen, 2014). My first three participants introduced me to some of their Vietnamese friends, who later became my participants. This technique was helpful and timesaving for me. Finally, my supervisor advertised my study in postgraduate courses which she taught and where there were Vietnamese students.

Students who had been in Aotearoa-New Zealand for a time longer than six months were regarded as eligible for this study. Selection decisions were made on a case-by-case basis. Students who met the selection criteria and showed an interest in participating in the study were approached.

\section{Participant profiles}

The master's students were from three master's programmes at VUW. The programmes were paper-based and one-year and a half long for the twinning students. None of the students had visited New Zealand prior to their studies. Seven (Lan, Bao, Thanh, Vy, Tam, Ly and Trung) were from the TESOL (Teaching English to Speakers of Other Languages) twinning programme, a joint programme between VUW and a Vietnamese university. There were around 15 Vietnamese students in this programme. They got an Aotearoa-New Zealand degree after successfully completing the first part of the programme in Vietnam and the second part in Aotearoa-New Zealand.

Most of the participants were in their twenties. All were either teachers in Vietnam or hoped to find a teaching job or a job in educational institutions after finishing their degrees, except for Hai, who was working as an official in a government-owned company. A summary of the participants' information is provided in Table 4.1 (pseudonyms have been used to protect their identity). 
Table 4.1

Profiles of the participants

\begin{tabular}{lllll} 
Pseudonym & Gender & Field of study & Marital status & Funding \\
Lan & Female & TESOL & Single & Self and family \\
\hline Bao & Female & TESOL & Married & Self \\
\hline Thanh & Female & TESOL & Single & Self and family \\
\hline Ngoc & Female & TESOL & Single & Scholarship \\
\hline Vy & Female & TESOL & Single & Self and family \\
\hline Tam & Female & TESOL & Single & Self and family \\
\hline Ly & Female & TESOL & Single & Self and family \\
\hline Trung & Male & TESOL & Single & Self and family \\
\hline Tuan & Male & Social Sciences & Single & Family \\
\hline Hai & Male & Political Science & Married & Scholarship \\
\hline
\end{tabular}

As indicated in Table 4.1, there were differences in the students' source of funding. Bao funded herself as she had worked for years and had savings. Most of the young students sought financial support from their extended families and had a paid work in Aotearoa-New Zealand to contribute to their tuition fees and living expenses, except My and Hai who were on government scholarships. Tuan, My and Hai did not have a paid job during their time in Aotearoa-New Zealand and relied on either family or scholarships.

\section{Data Collection Procedure}

Based on the research topic and the qualitative-oriented research method, data were collected through in-depth semi-structured interviews which are appropriate to this phenomenological research (Johnson \& Christensen, 2014; J. A. Smith et al., 2009).

A sequence of questions, which might vary from one participant to another, explored the participants' lived academic, social, and personal experiences. An interview schedule in IPA can be easily adapted to different circumstances (J. A. Smith, 2017). In this study, variation occurred because some preferred to talk about a particular aspect of their experiences before the others, or some happened to talk about a certain experience before I asked them. The interview questions began with general questions about their background, then moving 
on to more specific discussions about their experiences. I carried out an informal trial with three Vietnamese doctoral students at VUW to test out interview questions before the actual data collection. Coupled with this trial, I rehearsed for the interviews in a cohort group organised by my supervisor, which improved my interviewing skills.

After the students contacted me showing their interest in participating in the study, I emailed them back to arrange the time and venue for the interviews. Each participant was interviewed three times. There was a total of 30 interviews. Besides the interviews, I had some personal communication with them through email or Facebook to clarify details. The first interview, which was conducted at the end of trimester three, 2017, was to obtain their background information. At the beginning of this interview, I briefly explained to them the major points of the information sheet and consent form, such as the research purposes, the expected time commitment, and their rights as participants. They were told they would be offered a gift/koha in the form of a $\$ 20$ voucher at the end of each interview as an appreciation of their time. I got the consent form signed during this time if the participant did not change their mind about their participation. Besides, each participant was asked to choose a pseudonym from the list I prepared in advance. In the second interview, I explicitly asked them about their experiences. The third interview was designed to clarify certain points or to gain more detailed information about their responses in the second interview. Reflecting on the completed interviews, I made minor changes to the interview guide so that it worked better for later interviews. I finished the data collection in mid-April 2018.

The face-to-face interviews were conducted in Vietnamese to enable the participants to express themselves more easily and were recorded with their consent. Each interview lasted from approximately one hour to one and a half hours. Some participants were enthusiastic about my study and were willing to spend more time telling me about various aspects of their experiences. Sometimes, I found points interesting and wanted to know more about them, which extended the time. Whenever I saw an interview might take more time than expected, I talked to the particular participant about this to make sure that he or she was comfortable, and that I could keep the interview going on. 
Smith (2007) recommends that in IPA, an interviewer acts as a facilitator and guider rather than a dictator. Therefore, prompts for more and deeper details were only provided when respondents have difficulties answering the questions. I designed interview questions to encourage students to express their own thoughts, feelings, and experiences rather than what they thought I possibly wanted them to say. Whilst I was also a Vietnamese international student, I did not impose my pre-determined opinions during the interview process. The prepared interview questions acted as a guide that allowed me to both focus on aspects of the participants' experiences and further explore any particular experiences that emerged during the interviews. They could freely provide accounts of their experiences without being imposed on subjective ideas. My intention was not to influence or lead them because I wanted to get data that reflected their experiences.

\section{Data Analysis}

In this section, I describe the analytical procedure which is based on Smith and colleagues' work. This is followed by a discussion of the data translation process. I also provide a description of how the findings will be structured and presented in the following chapters.

\section{Analytical procedure}

I transcribed the interviews in Vietnamese after each interview round. Emerging findings from one round informed another. I printed the interview transcripts and used the traditional pen-and-paper method in the analysis. I analysed and interpreted the transcripts after the completion of all interview transcriptions, following the IPA guidelines by Smith and colleagues, which included six steps (Pietkiewicz \& Smith, 2014; J. A. Smith et al., 2009).

\section{$1^{\text {st }}$ step: Reading and re-reading the transcripts}

The first step involved reading each transcript and listening to the digital recording a number of times. This enabled me to correct the transcripts and get an overall feel for what participants said. New insights into the data were gained when I immersed myself in their narratives. My interactions with the participants as an interviewer and with the interviews as a transcriber enhanced my understanding of the students' accounts. These enabled identification and extraction of potential themes. Although in this process, I mainly focused 
on reading the transcript, I sometimes underlined or made notes in the left margin to record my thoughts about what seemed to be interesting or significant, or any queries and ideas that came to my mind.

\section{$2^{\text {nd }}$ step: Initial noting}

In the second step, I focused on the content, language use (e.g., interjections or code switching), context of the data, and indicators of participants' emotions, highlighting or underlining evocative or pertinent parts of the text, or any meaningful quotes that I found relevant to and useful for my study, and noting down every interesting point that I could see from the transcripts. In this way, I could keep myself close to the meanings the participants were making of their lived experiences. I was open to whatever emerged from the data. I worked with the transcripts of one participant at a time, going from one line to another. I made comments on the data, also in the left margin of the transcripts. At first, my comments were exploratory and merely described, summarised or paraphrased what the students said. As moving forward with the transcripts, I gradually had a better understanding of their accounts and could comment on associations or connections amongst what a participant said. Some of these initial comments were preliminary interpretations. At times, I returned to the notes made in the first step to remind myself of the previous thoughts. After reading the whole transcript and making preliminary notes, I proceeded to the next step.

\section{$3^{\text {rd }}$ step: Developing emergent themes}

Immersing myself in the data enabled me to get closer to the students' lived experiences. In the third step, I moved to a more abstract level of analysis when trying to understand the connections between the emergent themes. I read the commentaries that I made in the first two steps to find links and patterns. I also looked at specific chunks of texts and analysed them. By doing this, I moved through the hermeneutic circle (J. A. Smith et al., 2009). I developed themes by combining the codes I had created. I sought to capture the essential meaning of the participants' experience in these themes. I used keywords to document emerging theme titles and wrote them in the right margin of the transcripts. 
Two levels of themes in IPA are overarching/superordinate themes and sub-themes. In this step, emerging themes were developed, and would be bases for sub-theme and superordinate theme development in the subsequent step. Several emerging themes, which were not definitive, gave me general ideas about the concepts that I had identified. After completing this step with all three interviews of each case, I moved to the next one.

\section{$4^{\text {th }}$ step: Searching for connections across emergent themes}

This step involved grouping emergent themes by finding links or conceptual similarities through listing themes and mapping clusters of related themes. I listed the emergent themes from the interviews on a separate sheet, which enabled me to view all themes easily. Some of the themes were related to each other, helped to explain the others, or clustered together. Some of them became sub-themes while the others could become overarching themes. However, I recognised repeating patterns and observed new themes emerging; thus, new sub-themes or overarching themes might appear. New overarching themes were created by pulling together some of the categories identified in the previous steps.

In this step, I worked closely with the themes and the transcript. When the themes clustered, I returned to the Vietnamese transcript to make sure that a theme was consistent with what the student actually said. I drew on the two resources to understand what the student was saying.

After I had completed the above process and came up with a number of themes that appeared to reflect the essence of the student's experience, I created a table of themes. In this table, besides the themes, I provided relevant extracts and information about where in the transcript I could find them. The information included the extracts' timing in the original transcripts, the students' names, and the interview where the extracts were located. These details enabled me to easily find the extracts again in the recordings or transcripts.

At this point, I discarded some themes that were not prevalent or consistent with the structure of the emerging themes or did not have adequate supporting evidence. I was careful not to let my bias interfere with the selection process. I was aware that some of these omitted themes may re-emerge when the analysis progressed. 


\section{$5^{\text {th }}$ step: Moving to the next cases}

In the fifth step, I repeated the above process for each of the other nine participants' transcripts. Smith (2017) and Smith and Osborn (2008) emphasise that it is important to realise the importance of divergence (differences) and convergence (similarities) of the participants' accounts. I used the experiential themes obtained from the previous cases to help guide the analysis of the subsequent cases. I also acknowledged the emergence of new issues. I created tables of themes for each of the ten students.

\section{Final step: Looking for patterns across all cases}

The aim of IPA is to obtain an understanding of the participants' experiences (J. A. Smith, 2015). In line with this purpose, this step sought to identify the shared themes of their accounts. I compiled all the tables of themes into one and examined it to search for connections between them. I attempted to come up with general patterns that were relevant to all participants. I came to be conscious of the strong and weak connections. As suggested by Smith and Osborn (2008), themes were selected based on their prevalence within the data, the richness of the supportive evidence and the ability of the themes to illuminate different aspects of certain experiences. This process led to the identification of seven overarching themes and 25 sub-themes that will be presented in the following chapters. After establishing the patterns across all cases, I proceeded to write up the results.

\section{Data translation}

I conducted interviews in Vietnamese to enable the participants to express themselves in greater depth. I provided them with the transcripts and invited them to check for accuracy or adjust what they said or add things if necessary. After they agreed on the transcripts, I started to analyse them in Vietnamese. Al-Amer, Ramjan, Glew, Darwish, and Salamonson (2014) stated that exact word usage is important in phenomenological research where understanding participants' lived experiences and perspectives are the main purposes. In the translation process, meaning may be diminished or lost (Al-Amer, Ramjan, Glew, Darwish, \& Salamonson, 2016). Al-Amer et al. (2014) and Al-Amer et al. (2016) suggest that data be analysed in the source language and then themes be translated to enhance data trustworthiness. Following these suggestions, I transcribed and coded data in Vietnamese 
to keep their original meanings. I am aware that a good translation can add trustworthiness to data. Once I had completed the analysis, I decided on the quotes that would be used in the report and translated them into English. To provide a thick description to my study, translations of verbatim quotes were used. This is challenging because translators need to look closely at the intended meaning of the transcript (Al-Amer et al., 2016). I was the interviewer who communicated and interacted with participants. I brought these experiences to the translation process and carried out the translation myself, which could enhance original meaning retention.

Language proficiency is an important factor in translating data (Al-Amer et al., 2014). This involves understanding the source language and capturing subtle differences when translating data into the target language (Al-Amer et al., 2016). Therefore, translators' knowledge of the source and target language is critical to the quality of translation (Lopez, Figueroa, Connor, \& Maliski, 2008; Squires, 2009). Besides the linguistic ability, translators must be familiar with cultural values and beliefs of the studied group so that they can contextualise data (Al-Amer et al., 2014). I am an English language teacher with Vietnamese being my mother tongue. My knowledge of the two languages enabled me to realise the nuances of the two languages, and produce meaningful English versions of the Vietnamese transcripts while paying close attention to the meanings that participants wished to convey and the double hermeneutic continued into the writing up of the findings. Additionally, I discussed difficult concepts and checked the translation with my colleagues who are Vietnamese native speakers and English teachers so that an appropriate translation could be achieved.

\section{Presenting the findings}

I begin the findings chapters with Chapter Five on the patterns of the experiences across all cases. Although for the most part of the analysis, I seek to capture and present the essence of the participants' experiences, I want to maintain the idiographic approach that is critical to an interpretative phenomenological method (J. A. Smith et al., 2009). In this regard, I highlight the unique and shared features of their experiences drawing on interview data. In doing so, I acknowledge that each student has distinct characteristics and experiences. I 
then present the overarching themes (or themes for short) and sub-themes with supportive evidence from the interview data in Chapters Six, Seven, and Eight. The essence of the students' experiences is captured in Chapter Eight.

The analytic process revealed seven themes and 25 sub-themes. In these themes, I seek to understand and capture the essence of the postgraduate students' experiences living and studying in Aotearoa-New Zealand. Each theme is discussed and supported by extracts from the interviews. The first theme focuses on the issues facing the students in relation to their housing (e.g., finding accommodation, the impacts of living arrangements on studies, and how they negotiated relationships with their flatmates) and how they dealt with those issues. The second theme is related to their struggles with language and cultural differences in everyday settings. The focus of the third theme is on language difficulties facing them in academic settings. The fourth theme is about how such issues as unfamiliar teaching and learning styles, demands of course work, and part-time jobs may affect the students' learning experience. In the fifth theme, I focus on their desire to connect with their families. The sixth theme is about the factors that positively and negatively affected their ability to form meaningful relationships with others. The final theme is about the students' struggles to lead an independent life and how they learned to deal with everyday problems without their familiar network left in Vietnam. Overall, these themes capture the essential meaning of their living and learning experience. Table 4.2 below presents the structure of the themes and sub-themes.

\section{Table 4.2}

The structures of themes and sub-themes

\section{Themes}

1. Challenging living arrangements

\section{Subthemes}

1. Struggling to find a place to live

2. Unfamiliar rental housing market and vulnerable tenants

3. Support from flatmates

4. Issues with cramped living space

5. Privacy and personal security

6. Issues with flatmates and landlords/landladies

7. Learning to live with the other under the same roof 


\section{Themes}

2. Communication and cultural challenges

3. Language difficulties in the academic setting new academic environment
4. Struggling with the

\section{Subthemes}

1. Language difficulties

2. Struggling with cultural differences

1. Struggling with different accents

2. Struggling with writing assignments and reading materials

1. New teaching and learning styles

2. Heavy workload

3. Struggling with referencing

4. Impacts of part-time jobs on study

5. Resolving difficulties in the academic setting

5. Struggling with dislocation from home and familiar people

1. Homesickness and yearning for connections with families and friends

2. Family - concerns and motivation

6. Barriers and enablers

1. Enablers in forming relationships with co-nationals and in forming a meaningful other international students relationship

2. Barriers in forming relationships with host-national students

1. Lack of preparation for a new life

2. Struggling to survive

3. Becoming an independent person

4. The ability to take care of one's self

5. Financial independence and ability to manage budgets

\section{Ethical Considerations}

As suggested by Creswell (2014), it is necessary to show respect for the participants and sites. Therefore, prior to data collection, I obtained ethical approval from VUW Human Ethics Committee (see Appendix A). Ethical principles were strictly followed during the research process. I achieved written informed consent from interview participants. I made sure that they understood the information sheet and consent form before they signed it.

I informed potential participants in the invitation email that their participation in this study would be voluntary and they had the right to withdraw at any time. Students were assured 
that they would not be asked sensitive questions or any questions that could be traumatic. They could ask for a break at any time and declined to answer questions that they felt uncomfortable with. At times, the participants wanted to drink some water in the middle of the interviews. The reviewers from the VUW Human Ethics Committee (HEC) were concerned that my interview questions might ask participants to reflect on emotional topics and probe experiences that were upsetting. As suggested by HEC, during the interviews, I was watchful of signs of distress, and in the event that a participant was distressed I would check if they were comfortable continuing the interview, and what they would like to do. I prepared to offer participants information about services available within the university and in the community if appropriate. To do this, I took a range of brochures, information sheets, and contact details of support and advice services to the interviews. They included the counselling services and organisations, for example, Helpline and VUW Mauri Ora (Student Health and Counselling). The students were willing to answer my questions about issues they encountered. I had an impression that, in some cases, they might feel relieved when having a chance to share their unpleasant feelings with another person (me in this case) and to get their stories heard.

That the Vietnamese student community at VUW was small (around 264 as of December 2017) was identified as a potential issue because the participants could be recognised from their demographics and information. Participants were assured of their privacy and the confidentiality of their identity and the information provided. A pseudonym was used for each participant in the final report. Details that were unique or could identify a participant were either omitted from the final report, or subsumed within a broader category (e.g., name of hometown and family details) where appropriate, whilst still ensuring the original meanings of their accounts were not affected by such.

Additionally, for certain personal and cultural reasons, Vietnamese students might not feel comfortable sharing their personal experiences and problems with me - a stranger - in the first interview, especially when the interview was to be recorded. Therefore, I attempted to establish and maintain a relationship of trust with them in different ways. For example, in the first interview, I informed them about the purposes of the study and collected some background information. Then, I talked about some general topics that might have been of 
interest to them. We met at places (e.g., in a café or a meeting room in the library) that were suitable for recording, convenient for them, and where they felt comfortable to share and discuss their personal experiences.

In recognition of the importance of retaining students, their time commitment, and Vietnamese cultural practices, I provided participants with a gratuity (e.g., a small gift) at each interview. Generally, for the Vietnamese, it is a cultural norm and is courteous for people to give moderate gifts to those who help them or do something for them.

All data were electronically stored on my personal computers with password protection. Hard copies of data, such as printed transcripts and consent forms, were kept in locked file cabinets in my office. As required by HEC, the consent forms will be held for five years, and the participants' identifiable information will be destroyed one year after the completion of the thesis. Other research data, such as the interview recordings and transcripts, will be destroyed one year after the completion of the thesis.

\section{Trustworthiness}

Validity in qualitative research refers to 'trustworthiness' (Johnson \& Christensen, 2014). Trustworthiness of a study is critical to its value (Creswell \& Poth, 2017). Various strategies were utilised to ensure the study's validity. One of the criteria of trustworthiness is credibility, concerning the extent to which researchers understand and interpret respondents' views (Creswell, 2014; Johnson \& Christensen, 2014).

To ensure the credibility of the study, I employed various strategies. Member checking was used throughout the interview process and data analysis to validate my interpretations of participants' responses. I frequently asked my participants to clarify their responses and checked if I understood their perspectives correctly. I sent them the transcripts and asked them to check if the transcripts accurately reflected what they wanted to say. In writing up my findings, I have employed low-inference descriptors or verbatim quotes to reflect participants' thoughts and feelings (Johnson \& Christensen, 2014). My supervisors as outsiders challenged my assumptions and interpretation of data. I utilised extended fieldwork (Johnson \& Christensen, 2014, p. 303) by interviewing participants three times. 
Negative-case sampling (Johnson \& Christensen, 2014) was used to purposefully seek examples that oppose my expectations and explanations. Additionally, I have provided an audit trail through my detailed explanation of data gathering, analysing, and interpreting procedures. I have included a description of my participants, and a rich description of the research context (Creswell, 2014) so that readers can make judgement on transferability.

\section{Reflexive Positioning}

I was aware that, as a Vietnamese international student, I was also experiencing the fluid and complex acculturation process which may have impacted my position as a researcher. Therefore, I tried to be critically self-reflective of my personal background and position and how this might influence the process and conclusions (Creswell, 2014; Johnson \& Christensen, 2014).

Being an international student like the participants was an advantage in this research. Within the qualitative paradigm, researchers are viewed as co-creators of the data (McGrath, Palmgren, \& Liljedahl, 2019). My experiences and knowledge were important during the interviews because they aided my understanding of their context and stories, which were helpful in the data collection and analysis processes. Based on my knowledge, I actively invited them to provide in-depth information while keeping myself open to significant aspects of experiences that emerged from their stories.

Despite such benefits, it was challenging to manage my roles as a researcher and a Vietnamese international student. During the interviews, I positioned myself as a researcher rather than a student. For example, I did not share my own experiences so as to ensure that participants' responses were not affected by my sharing. My experiences and understanding of international students' experiences were recognised and identified during the research process. I tried not to allow my preconceived notions about international students' experiences to affect the participants' responses.

I was conscious of the possible way in which my age and gender might impact on the conversations between me and the participants, and thus on their responses. As a male student, I potentially had different experiences from my female counterparts. In some 
situations, female participants might not feel comfortable sharing certain aspects of their personal experiences with me. I was older than most of the participants, which potentially impacted on how they positioned me. For example, they might have perceived me as an academic who could comment on their stories or knowledge. To mitigate potential issues emerging from such matters, I attempted to build rapport with them and to create a positive atmosphere for them to share their stories. I was mindful not to pose questions that could make them think I was 'too curious', and not to make any comments or behaviours that could be viewed as judgmental.

\section{Chapter Summary}

In this chapter, I have presented the design of my study. Secondly, my research paradigm was presented, including my methodological, ontological, epistemological and axiological assumptions. Thirdly, my methodology was described with a focus on the concept of phenomenology, the philosophical foundations of phenomenology, the concept of IPA and its critiques. I then discussed the reasons for choosing IPA instead of other qualitative approaches. Fourthly, participant recruitment and profiles were detailed. Fifthly, I described the procedures for data collection and analysis. Data was analysed based on the six-step procedure proposed by Smith and colleagues. Finally, the chapter concluded with a consideration of ethical issues and trustworthiness. In the next chapter, I present the students' individual experiences and highlight the distinctive aspects of these experiences. 


\section{Chapter 5 | Individual Experiences: A Homogeneous but also Heterogeneous Cohort of Students}

Everything's a story.

You are a story - I am a story.

- Frances Hodgson Burnett

The purpose of this chapter is to capture the essence of individual experiences. I highlight the unique and shared features of the participants' experiences drawing on interview data. By providing an individualised story of each participant, I acknowledge that they had distinct characteristics and experiences.

Although each participant has a different way of making sense of their experiences resulting in different narratives, there are common themes emerging from their stories. Besides providing separate accounts of their experiences, I group them into four themes - longing for home and the feeling of not belonging, challenging living arrangements, struggling with a new learning environment, and surviving and thriving - based on the similarity in their experiences.

Apart from presenting the experiences of individual participants, I provide additional information, including their ages, family contexts, prior jobs, and their motivations to study overseas. These details will provide a contextual foundation for understanding the interpretative commentaries in the subsequent chapters.

\section{Longing for Home and the Feeling of Not Belonging}

This theme, which draws on the experiences of three participants: Bao, Tuan, and Vy, highlights their feelings about where they belonged and how connected they felt with people in Aotearoa-New Zealand and Vietnam. Bao felt an age gap distanced her from her Vietnamese classmates in Aotearoa-New Zealand and missed her children in Vietnam. Tuan missed his hometown, family, and loved ones in Vietnam and did not have friends in 
Aotearoa-New Zealand. Vy encountered many problems in daily life and felt that the country was not a place for her.

\section{Bao}

Bao was a TESOL student whose children stayed in Vietnam. Previously, she worked mainly as an interpreter, but hoped to establish a career as an English teacher after completing her studies abroad. Bao described herself as a mature, introverted, and old-fashioned person. She said that in Vietnam, it was not common for a woman of her age to be studying towards a master's degree. This suggests that she was an independent and brave woman.

Being both a mother and student made Bao's experiences different from others. Living in another country without her children was a big challenge for her. While one year in Aotearoa-New Zealand might not be a long time for some students, it was a long time for a mother like her. She missed her children and often talked to them on Facebook or Skype. One of the challenges was the difference in time zones between the two countries. Vietnam is six hours behind Aotearoa-New Zealand during non-daylight-saving months, and five hours during daylight-saving months. When her children in Vietnam came home after school, it was usually her bedtime. This limited her time with them, usually to around an hour. Because her children were involved with extracurricular activities on certain evenings of the week, she was not able to talk to them on those days. She had to negotiate a convenient time with her family members and children so that she could talk to them. "I left my children at home. [...] I always feel like I have left part [of myself] in Vietnam", Bao said. Her mental and physical health were affected by her homesickness. These emotionally draining experiences sometimes overwhelmed her. She could not focus on her studies and got sick. She described these stressors as "bacteria" and her reaction to this as "having a weak immune system".

It was due to these circumstances that she felt insecure and not belonging in Aotearoa-New Zealand. She saw herself as a tourist in the country, and her feelings of insecurity were strongly associated with health issues. Although she had Vietnamese classmates/ flatmates, she was conscious of an age gap between them and saw herself as part of an older generation from them, being closer in age to their parents. While she appreciated their 
company and assistance with difficulties, she felt that she failed to form meaningful and fulfilling relationships with them.

Being separated from her children significantly impacted Bao's life and studies. Her children were her motivation to advance her education. She would have felt guilty if she had not had good study results. She said that a woman at her age would be most concerned about "the relationship she left in Vietnam", which indicated her children. She wanted to complete her studies quickly to return home and meet her children.

\section{Tuan}

Tuan was a mid-twenties-year-old social sciences student, who described himself as a person who did not like being away from home. He was the younger of two siblings and the first member of his family to get university education. He did not have many close relationships. In the new and unfamiliar environment, he was able to establish relationships with people around him but not confident about maintaining them. These worries affected the way he socialised and limited his friendship networks. He spoke of his academic journey in Aotearoa-New Zealand as moving out of his comfort zone and was aware that it might be difficult for him. He wanted to spend a short period of time overseas to challenge himself and develop his language skills.

Whilst he had never lived away from his family, he believed that he was able to take care of himself, cooking, and organising things in his daily life. Unlike many self-funded students who attempted to find a part-time job to support themselves, he chose not to be employed. His parents had carefully prepared a financial plan to support his studies and living. They wanted him to be able to fully focus on his studies.

Being away from home, he felt homesick and lonely. He wished to return to Vietnam, leaving everything in Aotearoa-New Zealand behind. However, he refrained from doing so and buried himself in his studies to forget these feelings. Academic achievement was important to him, and he motivated himself by reminding himself about why he was here, and what he wanted to achieve. Thinking about the family reunion he would enjoy after graduation also helped him overcome his homesickness. 
Whilst other participants did not return to Vietnam during their time in Aotearoa-New Zealand because their programmes were of short duration and they wanted to spend time travelling around the country with their friends, Tuan returned to Vietnam once. "The thought of returning home helps me overcome difficulties," he said. In Aotearoa-New Zealand, he was the only Vietnamese student in his class. He rarely socialised and usually stayed home. Because his final course was online, he decided to leave for Vietnam, thinking that he could attend the course at home. "I don't think that life here is suitable for me", Tuan said. On his computer, he had a folder named Vietnam, which reminded him that he could return home soon.

\section{Vy}

Vy was in her early twenties and was studying TESOL. In Vietnam, she lived with her parents and older sister. Before leaving for Aotearoa-New Zealand, Vy worked as a part-time teacher in English centres and at a university. While she described herself as not being a sociable person, she was open to new relationships. Despite loving to meet people and experiencing new things, she viewed herself as an introverted person. In Vietnam, she spent most of her time at home and had few friends.

Partly driven by a desire to transform her personality and experience the outside world, Vy decided to study overseas. While she thought living away from home was interesting, she was worried that she could not live independently. In Vietnam, her parents took care of everything so that she could focus on her work. In Aotearoa-New Zealand, she had to do everything herself and work part-time to support her studies, which she found challenging.

Vy's difficulties in finding accommodation began on her arrival in Aotearoa-New Zealand and again six months later. She spent the first week looking for accommodation. She stayed in her first house for 6 months, then the owner sold the house and she had to move out. She and her flatmates stayed in a hostel for a month before finding a new place to live. Encountering these challenges, she said that she just wanted to return to Vietnam after completing her degree. She wanted stable accommodation, where she could feel rest assured to study and work, but not to worry about it all the time. 
Language issues contributed to her isolation and feelings of not belonging. In Aotearoa-New Zealand, Vy had worked at two places, one with mainly Asian co-workers, and the other with New Zealanders. She found it hard to open up when using English. Language issues impacted on her ability to socialise. She could get on well with her Asian co-workers, but had difficulties establishing meaningful relationships with the local ones. She also felt that she did not share a common ground with the New Zealand co-workers. Thinking that she could overcome these problems, she just focused on working instead of socialising.

Life in Aotearoa-New Zealand was a constant struggle and an endless cycle for $\mathrm{Vy}$. She said, "living here has never been easy. Problems occur all the time". When she managed to solve one problem, another occurred. Encountering difficulties and continuously feeling distressed and exhausted left her feeling that she had no place in the country. "This is not my home", she said. When asked why she wanted to return to Vietnam, Vy said she wished to see her parents, eat Vietnamese food, and be surrounded by the people she felt emotionally connected with. Aotearoa-New Zealand did not give her such feelings.

\section{Struggling with Living Arrangements}

This theme involves the participants' difficulties with securing accommodation and their negotiations of relationships with their flatmates. It draws on the experiences of two participants: Lan and Hai. Lan struggled with finding accommodation when she first came to Aotearoa-New Zealand. Both Lan and Hai attempted to build and maintain harmonious relationships with their flatmates, who were either from Vietnam or other countries.

\section{Lan}

Lan, who was in her early twenties, was a TESOL student. She was full of energy. Lan described herself as a laid-back, but sometimes conservative and straightforward person with strong character. Since she was 15 years old, she had lived with her sister in a city far from her hometown for study. She believed this helped her to become independent from an early age. She was able to take care of herself and make decisions about her life. Despite this, she said that she still had to ask her family for advice or permission for important things (e.g., overseas study). 
Finding accommodation was challenging for Lan. She was desperate for accommodation for the first two weeks after her arrival, which she described as "super tiring". She and her classmates had to quickly find a place to live because her studies were about to start. Six of them decided to share one hostel room until they found an affordable flat to rent. Worrying about accommodation and living in an unfamiliar environment strongly impacted on her feelings and eating habits. Although she was not a picky eater and eating had never been a concern for her, she did not feel like eating during that time. Reflecting on her experiences, she advised that "If you don't know anyone here and haven't found a place to live, you should stay in university Hall. You can have a decent life there". Finally, she and her classmates found a house that suited their needs, which made her "extremely happy". She lived there throughout her time in Aotearoa-New Zealand.

Lan lived with her Vietnamese classmates, which made her feel safe. There were advantages in having co-national flatmates. For example, they understood and were able to help each other. She shared: "Looking back, I was lucky to have my friends. If I had been alone, I wouldn't have survived the journey of finding accommodation. I was really stressed out. It was difficult to find a house to live". However, it was not without problems because people from the same country do not necessarily have the same lifestyles, attitudes, and behaviours. Sometimes, her flatmates were noisy. Dealing with these problems, she emphasised that "No one should stick to their own ego." For her, sharing a flat with many people required everyone to change. She had to adjust her daily routines and be considerate of others' needs.

\section{Hai}

Hai was a late-twenties student from the School of Political Studies, who described himself as a complex person. He married a few months before leaving Vietnam. Despite being reserved, he saw himself as a person who loved to experience and explore new things, meet new people, and make new friends. He felt that he lacked self-confidence. He said that at times he could not open up and seemed to cover himself with "a shell". At the same time, he identified himself as a self-reliant person with life experience. He had lived and worked in different regions of both North and South Vietnam, which gave him opportunities to 
experience the diversity of Vietnamese cultures and made him mature for his age. He was on a Vietnamese government scholarship. Although he did not worry about financial issues, he worked part-time during his first few months in Aotearoa-New Zealand. Then, he decided to stop working to focus on his studies.

Hai developed close relationships with flatmates from other cultures which made him an outlier among the participants. In his own words: "There were some people from other countries who I stayed with for just three months. But after moving out, we still keep in touch and sometimes have parties together. I feel that I was successful [in making friends]". His extensive experience of living and working in many regions in Vietnam and spending time with people from all walks of life, helps him understand and engage with people from diverse cultures. For him, what made a difference was to be open to diversity, and genuinely want to make friends.

Although Hai valued his relationships with flatmates, he experienced problems with them because of different lifestyles (e.g., partying and making noise). Sometimes, they seemed unaware that he needed a quiet space for study. Rather than talking to or confronting them, usually, he would go to the library and spend his whole day there. He highlighted the capability to control oneself and understand cultural differences. While he strived to accept such differences, he ended up moving out. "When I stayed with people from other countries, there were cultural differences. After moving out to stay with a Vietnamese family, I felt more comfortable because we share a cultural background", he said.

\section{Struggling with New Learning Environments}

In unfamiliar academic settings, the students encountered a range of difficulties. This theme captures such experiences and includes two students: Ngoc and Tam. Both had a hard time with the English language and found it difficult to keep up with their classmates.

\section{Ngoc}

Ngoc was a TESOL student, who was in her mid-twenties. She was the youngest child and lived with her parents when she was in Vietnam. While neither of her sisters finished 
university, her brother was a lecturer and was studying for a doctorate. Ngoc worked as a lecturer before leaving for Aotearoa-New Zealand. She studied on a Vietnamese government scholarship. She was not burdened with financial issues but was required to work for her home university for a few years after graduation. She did not have a part-time job although she thought she might like one. Not working helped her focus on her studies.

An elderly close relative passed away just one day before her departure. However, her journey had been set up, and she could not stay home but knew that she needed to go. During the interview, she became emotional, lowered her voice, and paused a few times when talking about the flight and the incident.

Ngoc saw herself as an introverted person and she tried to be friendly. In conversations, she did not talk much, but was relatively sociable. Although she enjoyed talking with others, she had few friends in Aotearoa-New Zealand. Her Vietnamese friends commented that she was a little shy and timid, and "did things slowly". She was adaptable to new environments, and after nearly a year, she became more open to new relationships.

Ngoc was strongly motivated in her studies. Unlike some Vietnamese students who were somewhat uncertain about their future jobs, she had a job already. She wanted to build her career in academia. She expressed a strong desire to obtain a scholarship and complete a $\mathrm{PhD}$ in the future.

Ngoc reported on her worries about language issues. She had difficulties with the different accents of Aotearoa-New Zealand lecturers or those who were originally from other countries, which affected her understanding of lectures, especially during her first few months. She had a sense of self-doubt when confronting language difficulties. She thought she would never be able to use English in her studies. Ngoc recalled that she once sought advice from her friend who said that "Just take it easy. Gradually you will get used to it [the accent]." Hearing this, she was comforted.

Ngoc needed to learn many things in the new educational environment, which made her overwhelmed. She did not understand her lecturers' expectations for assignments and the information posted on Blackboard - an online platform used to support teaching. She then 
sought assistance from her Vietnamese friends who came to Aotearoa-New Zealand before her. Their advice made her more confident in her ability to overcome difficulties. After that, doing assignments was not a big concern for her anymore.

Reflecting on her learning experiences, she advised prospective international students that "before coming here, they should be well-prepared and seek information about courses and available support services. They should take advantage of those services". Apart from attending a creative writing club and being a member of the university recreation centre, she did not utilise other on-campus support services. Although she recognised the usefulness of such services in improving international students' learning experiences, she could not arrange her time and did not feel the need to seek professional assistance.

\section{Tam}

Tam was in her early twenties and lived with her parents when she was in Vietnam. She described herself as a traditional, careful, and meticulous person. She did things in accordance with social norms and always tried her best to finish the work that she was assigned. She thought she was introverted, not open to other people, and a little bit cautious when making new friends. She hoped that she would become a more open and broadminded person who was able to accept new things after completing her studies.

Tam started the TESOL twinning programme about 6 months after completing her bachelor's degree. At that time, she was working as a part-time teacher at a foreign language centre and as an employee at a local supermarket. Once she began the twinning programme, she quit the job at the supermarket, but worked at the language centre until she departed for Aotearoa-New Zealand. She experienced different work environments, and finally decided on becoming a teacher or working in the educational sector.

Language issues had a negative impact on Tam's feelings. She reacted with a feeling of passivity and "decided not to say anything" when facing difficulties in expressing herself. Language difficulties affected her ability to understand academic texts. She found it challenging to keep up with her classmates who were local students. She needed more time to complete the required readings. She was self-conscious about her difficulties and spent 
time on self-directed learning. She studied at the university for the whole day and just returned home to sleep.

As a self-funded student supported by her family, Tam felt a need to have a part-time job to contribute to her expenses. However, she recognised potential negative impacts of working on her studies. She recalled a period when she usually felt exhausted and sleepy but "needed to drag herself to class" because of not having enough time to rest.

\section{Surviving and Thriving}

This theme, which includes three participants: Thanh, Ly and Trung, focuses on how difficulties in their daily lives helped them to become independent. Thanh developed independent living skills and was able to take care of herself. Ly gained more freedom and could decide for her life. Overseas study gave Trung opportunities to open his horizons and seek out new experiences that he did not have in his home country.

\section{Thanh}

Thanh is a mid-twenties TESOL student, who worked as an English teacher at a Vietnamese university. Before that, she worked in language centres where she taught learners of various age groups. She described herself as being a little bit introverted. However, this did not prevent her from socialising and extending her friendship network. When someone took the initiative to interact with her, and she felt that person was on the same wavelength, she would attempt to maintain the relationship.

Her parents were protective of her and did everything for her when she was home. Therefore, they considered overseas study as an opportunity for her to grow and become an independent person. She understood that her parents had expectations for her, but they did not let her know for fear of putting pressure on her.

It was challenging for Thanh to live without her family, especially during the first few months. For the first time in her life, she needed to take responsibility for her own life. However, she lacked the skills required for an independent life, such as cooking or taking care of herself properly. During those early months, she mainly lived on boiled vegetables. Many 
problems such as the stress of study, the cold weather, and losing weight, collectively impacted on her health, leading to her being briefly hospitalised.

Living away from her family forced Thanh to learn to be independent. Although appreciating her classmates' companionship throughout her time in Aotearoa-New Zealand, she felt a strong need to be self-reliant. "Because I am in New Zealand by myself, I need to find ways to deal with problems myself," she said. She learnt to organise her life and focused on what mattered most. She said, "I worked out what is most important to me and focus on it. I just left other things out." Recognising the role of healthy diets, she improved her cooking by watching YouTube videos. She learnt to take care of herself through observing how other people dealt with health issues. Reflecting on her hard times, she commented that they had "brought about a lot of changes" to her. She now felt that she was "no longer a child" and could take responsibility for her life.

\section{Ly}

Ly was a mid-twenties TESOL student, who saw herself as an active person. She loved to get involved in different things and not to stay in one place for long. "I want to have good study results, so I work hard and prioritise my studies," she said. When she was in Vietnam, she was not interested in networking and expanding her social circles. However, in AotearoaNew Zealand, she found that she could not live by herself. She worked in a restaurant, where she came to realise that she needed several skills for her work. Being in a new work environment, she had to learn from others, especially her colleagues. Over time, she became aware of the importance of networking and learnt how to establish relationships with colleagues.

Life in a foreign country allowed Ly the freedom to make decisions. In Vietnam, she was not responsible for anything except her studies and work. She felt that her life was constrained because her parents seemed to see her as a little child who needed protecting and managing. Whilst they wanted her to live with them after completing her studies, she wished to leave home and have her own life. In Aotearoa-New Zealand, she learnt to take care of herself and takes responsibility for her life. "Living here helps me become more independent. I had to do everything on my own. I have learnt to manage my time for studies 
and work. I have tried my best and I value my transformation," she said. She decided to stay in the country for a few years after graduation. This suggested that she enjoyed her freedom and independence and could decide her life choices.

\section{Trung}

Trung is an early-twenties TESOL student who comes from a humble background. He described himself as a friendly person who loves to make new friends and mingle with people. He loves to explore new places and experience new things. He can adapt well to new environments. He used to travel to Singapore on his own, which may not be common for a young man from a remote area in Vietnam to do.

Trung emphasised the importance of health and financial independence. He believed that people should be able to take good care of themselves, especially when their families were not beside them. He knew his own health conditions and got appropriate medication. He tried to balance his life, for example, he rested and ate appropriately to protect his health. Having a less privileged background, he was aware of the difficulties facing his family in supporting his studies. He worked hard to support himself in Aotearoa-New Zealand. During the first few weeks, he was new to everything, and it was hard for him to find a job. Once employed, he was able to cope with his financial problems.

Previously, Trung moved to a major Vietnamese city for university education and lived there for 6 years, which helped him become an independent person. He talked about his journey from Vietnam to Aotearoa-New Zealand as a journey of "getting out of bamboo hedges of villages". Bamboos are usually grown at the edge of a village, which forms a typical landscape of Vietnamese villages. In some contexts, this landscape symbolises the love for homeland, but in others, it represents the restriction of knowledge (L. T. Tran \& Marginson, 2014). Traveling beyond the village bamboo can help people to learn about the outside world. In Vietnamese tradition, the notion of mobility is associated with the desire to expand one's mind and knowledge. In other words, mobility is driven by an aspiration for selfdevelopment. In deciding to study abroad, he wanted to continue his journey of exploring the world and improving himself. Trung believed that living in another country enabled him to have new and fresh experiences. He decided to stay in the country after completing his 
degree, which suggested that his journey was incomplete, and he wished to continue it. "I will live in the place where I feel comfortable and happy and where there are opportunities [for personal development]," he said.

\section{Chapter Summary}

The analysis revealed four themes of the Vietnamese students' experiences. The first theme was about the students' (Bao, Tuan, and Vy) homesickness and longing for home. Because of these feelings, they did not have a sense of belonging in Aotearoa-New Zealand. Bao's feelings were largely associated with her children back home; Tuan felt that life in the country was unsuitable for him; and $\mathrm{V} y$ found issues in everyday life overwhelming, and they were like an endless circle for her.

The second theme was about the students' (Lan and Hai) struggles with living arrangements. Lan had a hard time finding affordable accommodation where she could stay with her Vietnamese friends. Hai shared his house with people from different countries. Both attempted to accept and establish harmonious relationships with their flatmates by keeping their emotions under control and avoiding possible tensions.

In the third theme, which included two students: Ngoc and Tam, I captured the students' struggles with the new educational environment. Both had language difficulties in class and felt bewildered in the new environment. Ngoc was overwhelmed because she had to quickly learn many new things. Tam had difficulties in keeping up with her flatmates.

In the final theme, I described the students' (Thanh, Ly and Trung) self-development when they went through difficulties. Thanh acquired practical daily living skills and became more independent; Ly felt that living away from her family gave her freedom and allowed her to take responsibility for her life; and Trung viewed his overseas study as a journey to expand his mind and open up new opportunities for his life.

In the next chapter, focusing on the students' experiences in the everyday setting, I will present findings on their struggles with living arrangements and difficulties related to language and cultural differences. 


\title{
Chapter 6 | Initial Settlement Difficulties and Learning to Live Alongside Others: Living Arrangements, Communication and Cultural Challenges
}

\author{
Vạn sự khởi đầu nan. \\ Many things begin with difficulties. \\ - Vietnamese proverb
}

...and so, their journeys began...

Embarking on a long journey could be a challenge. Finding a place to live was one of the students' first concerns once they arrived in Aotearoa-New Zealand. In this chapter, I start with an introduction to their living situations and then focus on two overarching themes: challenging living arrangements, and communication and cultural challenges. In the first theme, there are seven sub-themes: struggling to find a place to live, unfamiliar rental housing market and vulnerable tenants, support from flatmates, issues with cramped living space, privacy and male flatmates, issues with flatmates and landlords/landladies, and learning to live with others under the same roof. The second theme has two sub-themes: language difficulties and struggling with cultural differences.

I highlight the participants' vulnerability in relation to their living circumstances. The students encountered difficulties in finding suitable accommodation. They each sought to negotiate relationships with their flatmates. Language difficulties were observable, especially during their first few months in Aotearoa-New Zealand. Most of them struggled with unfamiliar cultural conventions and norms, which had an impact on their feelings and made them feel like outsiders.

In this study, the term 'roommate' indicates a person occupying the same room as another while 'flatmate' is used to refer to a person who shares a flat or house with others. 
A paper based on a good part of this chapter has been published and is entitled: 'The experiences of Vietnamese students in New Zealand: A new country - a new home' (Ho, 2020). The themes in this chapter have additional data that were not presented in the paper. The three themes - 'unfamiliar rental housing market and vulnerable tenants', 'language difficulties' and 'struggling with cultural differences' - are not included in that publication.

\section{Living Situations of the Students}

The participants were not accompanied by families and had limited overseas experience. Ly had attended a student exchange programme in Indonesia for two weeks. Bao's husband and children remained in Vietnam, as did Hai's wife. Table 6.1 presents their living situation changes, with a focus on their flatmates:

Table 6.1

Living situation changes of the students

\begin{tabular}{|c|c|c|c|}
\hline & \multicolumn{3}{|l|}{ Living situation } \\
\hline & Time 1 & Time 2 & Time 3 \\
\hline Lan & \multicolumn{3}{|c|}{ Lived with 4 Vietnamese friends throughout the time in Aotearoa-New Zealand } \\
\hline Bao & \multicolumn{3}{|c|}{ Lived with 4 Vietnamese friends throughout the time in Aotearoa-New Zealand } \\
\hline Thanh & $\begin{array}{l}\text { Vietnamese and New } \\
\text { Zealand flatmates (a } \\
\text { couple) }\end{array}$ & $\begin{array}{l}1 \text { Vietnamese landlady } \\
\text { and } 1 \text { Vietnamese } \\
\text { flatmate }\end{array}$ & $\begin{array}{l}3 \text { Vietnamese } \\
\text { classmates }\end{array}$ \\
\hline Ngoc & $\begin{array}{l}3 \text { Vietnamese and } 1 \text { English } \\
\text { flatmate }\end{array}$ & 4 Vietnamese friends & \\
\hline Hai & $\begin{array}{l}\text { A Vietnamese family } \\
\text { (parents and child) }\end{array}$ & $\begin{array}{l}5 \text { New Zealand and } 1 \\
\text { English flatmate }\end{array}$ & $\begin{array}{l}\text { A Vietnamese family } \\
\text { (parents and child) }\end{array}$ \\
\hline Tuan & \multicolumn{3}{|c|}{ A Vietnamese family (parents and a child) } \\
\hline Vy & 4 Vietnamese flatmates & 5 Vietnamese flatmates & \\
\hline Tam & \multicolumn{3}{|c|}{5 Vietnamese flatmates (including mother and child) } \\
\hline Trung & \multicolumn{3}{|c|}{$\begin{array}{l}\text { Four flatmates (1 Vietnamese student, parents (Vietnamese and New Zealander) } \\
\text { and child) }\end{array}$} \\
\hline Ly & University hall & 4 Vietnamese friends & \\
\hline
\end{tabular}

As illustrated in Table 6.1, six of the students (Thanh, Ngoc, Hai, Vy, Trung and Ly) changed their accommodation more than once over the year. Ly was the only student who had lived in a university hall. Their reasons for moving included the desire to flat with Vietnamese friends, to be nearer to campus, unpleasant experiences with current living arrangements 
(e.g., problems with flatmates and issues related to limited or overcrowded living spaces), and/or more affordable accommodation.

\section{Challenging Living Arrangements}

According to Scoop Business (2020), an Aotearoa-New Zealand independent news website, Wellington has the highest demand for rental property in the country, and in January 2020, the median rent in the city rose to $\$ 575$ per week for the first time. Such an unaffordable housing market posed significant challenges to the students. In this section, the discussion centred around their difficulties in securing accommodation, navigating the renting regulations, cramped living spaces, issues with people who shared their houses, privacy and personal security. Further, this theme focuses on how their co-national flatmates might assist them in their daily lives and how they negotiated relationships with their flatmates.

\section{Struggling to find a place to live}

This theme is about the challenges facing the students in finding accommodation. They all reported that affordability was an important factor in their choice of accommodation. When residing within the establishments of the university became impossible because of budget constraints, they turned to private accommodation as a solution. The students in the twinning programme arrived in Aotearoa-New Zealand around January. This was a time of high demand for rental accommodation in the country's major centres and it was challenging for them to secure accommodation. Finding accommodation was an integral part of setting up a new life because unsuitable living arrangements would affect their studies. Bao emphasised that despite her 18-month stay in Aotearoa-New Zealand, she needed to "set up a new life" and "to have a decent living".

Most of the students found it hard to find accommodation of their own choice. They lowered their initial expectations and compromised by limiting themselves to housing that was within their tight budgets. For financial reasons, none of them, except Ly, chose to live in a university hall. Thanh, for example, did not want the rent to be "a financial burden for her parents". There was a sense of desperation as they were aware that they should get accommodation organised quickly before their courses started. However, it was difficult for 
them as they did not know the options and had few friends in Aotearoa-New Zealand who could help them.

Finding accommodation in a foreign country can be a time-consuming and bewildering process. The students started their hunting for accommodation when they were still in Vietnam. The internet and networking sites (e.g., Facebook) enabled them to connect with homeowners or Vietnamese students in Aotearoa-New Zealand who could assist. They found houses online that they would consider renting upon arrival. They wanted to view the houses directly to make sure they met their needs. One used Google to measure the distance to the university. Despite this, she still felt the need to see the house in person:

The only thing that made me exhausted was looking for a house. The first two weeks made me super tired. [...] But actually, you didn't know if that place was near or far. You have never been there. Although you found some houses when still in Vietnam and Google the distance [to university, supermarket...], there are still some differences [between reality and Google result]. (Lan)

Challenges in finding suitable accommodation left the students with mixed feelings of marginalisation, frustration, desperation, and emotional and physical exhaustion. Unable to find houses to rent, some booked temporary accommodation in backpackers' hostels. While this strategy was effective, it was not what they expected. For Bao, this journey felt a hopeless one because nobody wanted to rent their houses to her. Trung was worried when he could not find accommodation after "contacting everyone he knew here for assistance" and felt that he could "become homeless". Being under pressure to settle down quickly to start classes added to his worries. The following extracts showed how the students attempted to make sense of their struggles.

I felt I was small, insignificant, and marginalised. I mean, I wasn't accepted. I felt hopeless because we couldn't manage to rent a house during the first week, although we found some when in Vietnam and had arranged to view them. (Bao) 
I was worried when I couldn't find accommodation. I didn't know where to go. I might become homeless. I had to carry my luggage with me almost every day. I was very tired. (Trung)

Temporary life in a backpacker hostel was difficult for some students. Vy felt trapped and oppressed in a hostel. In her own words:

I had to find ways to deal with problems. Like when I lived in a hostel, it was very hot and suffocating. Despite that, I had to get over that to study. (Vy)

The university accommodation service was not useful to the students as they did not wish to live on campus:

I think the service mainly focuses on students who wish to live in the halls. For those who want to live off-campus like us, I have a feeling that they just don't care. (Bao)

Eight of the students came to Aotearoa-New Zealand with others in their twinning programme. Coming in a group meant that they had someone who understood and was willing to help them with difficulties. This made them feel more secure. Some of them wanted to flat together, at least for the first few months until they were familiar with the new environment. Thanh, for example, initially wished to stay with her four friends but later found it impossible because she could not obtain a place to accommodate all five of them. She ended up leaving her group to find another place for herself:

I felt that I was alone in everything. We studied in the same programme, came here together, and decided to stay together in the same flat. But why couldn't all five of us stay together? (Thanh)

In Vietnam, the students worked through their university and an agent connected with it. This agent assisted them with their visa applications and provided them with information about Aotearoa-New Zealand culture, teaching, and learning. However, while the students were concerned about accommodation, little help was offered. All the agent said was that they had the option of living either on-campus or off-campus. Thanh lamented: 
They didn't help us rent a house or suggest a house for us. [...] We had to do everything by ourselves.

The information on the university's website about private accommodation was not of use to the students. They mainly found accommodation information on Trademe - a New Zealand online auction website, or through their personal networks.

Vy had a hard time searching for accommodation. Despite feeling depressed at times, she emphasised the need to get over problems and achieve emotional stability:

I felt very depressed when searching for accommodation. [...] It was very difficult. Even when I didn't have accommodation, I still had to study. There was a time when I felt very low but had to keep moving forward and keep my life in balance. (Vy)

After days of desperately finding accommodation, the students were exhausted. For fear of not being able to find a place to settle down and start their studies, they found themselves in a race against time. Some of them ended up choosing a place which they thought was neither good nor bad without thinking twice. What they needed at that time was a place to live. This was an uncertain time for them. In the following extract, Lan emphasised that finding accommodation was a weary journey which she wished to end.

We viewed the house in the afternoon and decided on it in the evening. We accepted whatever the price was because we were really tired. If we refused, we wouldn't know whether we could find another one. The rent was high, but we just stayed and ignored any problems. (Lan)

Fortunately, after staying in that house for a while, Lan and her friends found it a decent place to stay. Lan lived there throughout her time in Aotearoa-New Zealand.

This theme has depicted the students' need to settle quickly to begin their studies and their desperation for rentals. Apart from the fact that they arrived in Aotearoa-New Zealand at a time when there was a high demand for rental accommodation, there were other factors contributing to their challenges in finding accommodation and living arrangements, which are presented in the next section. 


\section{Unfamiliar rental housing market and vulnerable tenants}

This theme deals with the students' limited knowledge about the local rental housing market, renting laws, and tenants' rights. These affected their ability to find and secure accommodation and potentially made them vulnerable.

The students found the differences in the rental housing market between Vietnam and Aotearoa-New Zealand difficult to understand. The most cited difference was related to the renting procedures. "Things here are very different from Vietnam, so in the beginning, we were very frustrated", Lan commented. When Vy first arrived, she wanted to quickly settle into her new life to begin her studies. However, it took her a week to find suitable accommodation and another week to complete the paperwork before she could move in. This lengthy process was her "first shock" in the country. In the extracts below, there was a sense that she felt frustrated and bewildered by the complicated application procedure.

In New Zealand, people had extensive procedures [to rent a house] which took us a whole week to complete. We had to learn how to pay the bill and were required to have a credit card, which was complicated. In Vietnam, we could move in first and do the paperwork later and we could pay the power bill to the landlord. (Vy)

Vy changed accommodation once. She wanted to move out, but it was hard to find suitable accommodation. She commented that it was a "terrifying" experience for her. It was January and she "couldn't find anyone who wanted to rent their house" to her. She and her friends had to temporarily stay in a hostel for the whole month. Her situation was tricky because real estate agencies rented their houses for a full year, but, at that time, she just had six months left and had to leave before her visa expired:

The agencies wanted us to sign a one-year agreement, but we just had six months in New Zealand, so we couldn't. They even checked our visa. Fortunately, we finally found one who agreed to rent her house to us. (Vy)

In the same vein, Lan explained that it was difficult for her to find accommodation because of the unfamiliar renting procedure: 
The biggest difference was that if there were many people interested in a house, you had to complete a form and wait for your turn. It appeared that owners liked to authorise an agency to rent their houses. (Lan)

These problems were related to the inflexibility of real estate agencies in terms of the length of tenancy, the required paperwork, and the renting procedure. As indicated in the above extracts, these issues were "the biggest difference" and caused difficulties for them. It was challenging even for $\mathrm{Vy}$, who had been in Aotearoa-New Zealand for a year and had experience in finding accommodation.

Few of the students had knowledge of the local rental market. Ly reported that she stayed in the university hall for six months. Before she moved out, the staff at the hall talked to her about issues related to private rental accommodation (e.g., renting laws and tenants' rights). Similarly, Lan had some information relevant to accommodation prior to her departure. She commented that thanks to these detailed procedures, she was able to 'deal with problems that may emerge along the way'.

I learned about it before coming here. The Vietnamese university provided us with information and a booklet. Senior students [who were our friends] told us to research thoroughly about renting laws, fees, and tenancy agreements. (Lan)

Most of the students were unaware of policies and rights as renters, especially in the early months, and they were unmotivated to learn about them. Ngoc's head tenant was a Vietnamese student who oversaw everything related to the house. Therefore, she did not feel a need to learn about tenancy issues.

I know little about the rental regulations, policies, and rights as tenants in New Zealand. When I first came here, I stayed with a Vietnamese doctoral student who was the head tenant and she did everything related to renting. I didn't encounter any problems when living with her, so I didn't need to learn [about renting policies]. I stay here for more than a year, so I don't have to learn about such things. (Ngoc) 
Ngoc then moved to another house while a Vietnamese student tenant went back to her country for data collection. In fact, subletting space to others or renting from their Vietnamese friends or people whom they connected with (but were not homeowners) was a common practice among Vietnamese students. Head tenants might sublet spaces without letting agencies or landlords know because they wanted to keep housing costs down. The students lived within or around the CBD (central business district) which was close to their campus and workplaces. However, housing in such areas tended to be expensive, resulting in their desire to reduce costs. Ly left her hall and ended up moving to another house to live with her friends without a written agreement with the landlord. Also, the challenge in finding accommodation contributed to her decision on where to live. In the following extract, Lan talked about people who she knew and believed to be "illegal tenants", emphasising that such practice was common, and no one had faced legal trouble with that:

Only three people in the house have a written agreement, but they share their house with other three people, totaling six people in a house. It's common and no trouble has happened. (Lan)

Although Lan had some knowledge about renting laws, she seemed to underestimate the importance of following the laws. Eight of the ten students were involved in this type of renting at least once during their time in Aotearoa-New Zealand. In terms of tenants' rights, this could put the students at risk and make them more vulnerable than those who rented from a landlord or real estate agent. The rights of renters in these situations were complicated because the students did not necessarily have written tenancy agreements as required by renting law. There was a sense that they were unaware of the risks and thought this was normal because their friends were doing the same thing. In the case of Ngoc, as indicated in the extracts above, she rented from her Vietnamese friends twice because it was convenient, and she encountered no problems with it.

Ngoc was aware that there was a restriction on the number of people in a house but thought that it was unnecessary to set such a limit. Reflecting on her own situation, she said that she usually spent her whole day at the university and did not need a large bedroom because she just returned home to sleep. She noted that she could study more effectively in the 
university library than at home, where she probably did not have a place to study. Her house was noisy and overcrowded. Despite this, there was a sense that she could compromise a comfortable living space for the sake of reducing her financial burden.

\section{Support from flatmates}

Newly arrived international students may feel vulnerable in an unfamiliar country and experience some degree of emotional distress (e.g., loneliness or isolation) before finding their networks in a host country. In this study, the students reported few instances of such distress and could deal with negative feelings thanks to their flatmates' support. This shows the importance of such support in their lives.

Spending time with Vietnamese friends, classmates, and flatmates made a difference to the students' lives. Some lived with their co-national classmates, who provided them with emotional and practical support, and advice or assistance on health and medicine.

When I was sick, I let my Vietnamese friend know and she would give me some pills. She also gave me advice about what kinds of medicine I should take. (Ngoc)

Homesickness or loneliness was not always something that the students could deal with alone. Friends offered solace during difficult times:

When I was homesick, they encouraged me to get through. We might watch a movie or cook together to forget that feeling. ( $\mathrm{NgOC}$ )

Ngoc had friends that she enjoyed spending time with. For her, cooking with friends was not just about food but bonding with them and dealing with negative feelings. It deepened their connections and created a sense of community which comforted them. It was an opportunity for them to get together and catch up on each other's lives and studies. She made cooking into social and fun activities which helped to reduce her distress and temporarily "forget" unwanted feelings.

Moving around in an unfamiliar country might trigger feelings of insecurity for some students. However, as shown in the interviews, this could be resolved by travelling with a 
group of friends. Lan arrived in Aotearoa-New Zealand with her Vietnamese classmates which made her feel safe because they helped each other when needed.

When we went through Auckland customs, we were checked very closely because we carried food. So, we were late for our flight and were really scared. Luckily, we went in a group. Then, we stayed in a hostel together. That was our advantage. We felt safe because at least, we had someone to hold on to in a strange land. (Lan)

When Lan arrived in Auckland, she and one of her friends lost their luggage. At that moment, she realised the value of having travelling companions.

My friend and I lost luggage. We were confused and didn't know what to do. [...] I felt lucky because I was going with my friends. Before I came here, I always thought we had to go together all the time. That would make me feel safe. (Lan)

Thanh reported that she was hospitalised at mid-night, and her friends rallied around to care for her. One stayed with her that night although there was a deadline for an assignment the following day. The friends recognised that her family was not there and stayed with her until she was discharged. Thanh's situation might have got worse without her friends.

I felt lucky. [...] They took care of me carefully and didn't leave me. I was there with no relatives around. It was fine for them to just leave me there and went home, but finally, they didn't. I was grateful to them. (Thanh)

There was a strong bond between the students and their Vietnamese friends. Their relationship was established through spending time and having fun together:

We know each other better and can tell a joke on everything, even sensitive topics. [...] This helped me release stress. So, I want to have Vietnamese friends. (Bao)

Vietnamese traditional holidays sometimes made the students feel homesick. In Vietnam, Tết (Lunar New Year festival) is a time for family reunions. Hai felt lucky to stay in a house with a landlord who was originally from Vietnam because he could enjoy the traditional dishes for Tết: 
We had bánh chưng (square glutinous rice cake), bánh giò (pyramidal rice dumpling), and nem (fermented pork roll). It was really like Tet in Vietnam. I felt like I was at home, not in a foreign country. (Hai)

For Hai, being able to eat Vietnamese traditional dishes and having a decent Tết created a sense of family, or a sense of Vietnam right in Aotearoa-New Zealand.

Tam felt that her life in the country was easier than expected because she was living with her Vietnamese friends. Doing everyday tasks together and frequently having meaningful conversations had a positive impact on her feelings. Things became "simple" when she was with her friends. This helped her feel more comfortable with life.

I thought living here would be difficult, and I had to do everything by myself. But my roommate was very nice. We cooked and ate together. I think everything is very simple. [...] The advantage is that we can talk about everything in life and care about each other's feelings. (Tam)

Tam used the Student Health Service before but now she did not use it anymore because she could talk to her Vietnamese friends about her problems. Living and talking with them had a positive impact on her emotions and feelings. Her friends could also give her advice regarding health issues.

Previously, I studied and worked part-time at the same time. I felt exhausted. I was like, I couldn't catch up with my flatmates. I went to the Student Health service for psychological consultation once. I'm living with Vietnamese people, so I can also talk to them about my issues. They understand me because we're all students. Everything is getting better for me. I don't use the service anymore. (Tam)

Tam appreciated her friends' assistance. In the following extract, she emphasised that she could have a positive living experience thanks to their companions.

My life here is easy and comfortable because there are many Vietnamese people around me, with the same culture and thinking. [...] I'm lucky to live with them. They're experienced people, and I can learn many things from them. (Tam) 
This theme has described how the students' co-national networks, especially their flatmates, provided valuable assistance in their hard times and a comfortable environment for their daily lives. Many students accepted poor housing conditions and lived with their co-national peers for such benefits. The following section focuses on how the issues related to limited living spaces might contribute to their studies and everyday routines.

\section{Issues with cramped living spaces}

This theme is about the impact of limited living space on the students' lives and studies. Being in an unfamiliar country with limited budgets, they were aware that they had to save money for unexpected things which may happen (e.g., sickness). Therefore, they shared their flats or rooms with other people. This allowed for sharing of housing costs, such as weekly rent and utility costs. One of the obvious problems with living in crowded and confined share housing was the limited space for study. The students came to consider the library as a suitable replacement for a study space. While Bao found that studying in the library was a common practice for students, she still missed her spacious house in Vietnam where she could learn comfortably.

I don't have the comfort of home here. My home in Vietnam was spacious, and I had my own learning space. Everything. But in New Zealand, I'm living in a small flat. I have to go to the library when I want to study. There's almost no space to study at home. (Bao)

While increasing the number of flatmates was beneficial in terms of financial issues, it might compound existing problems. Bao implied that her living conditions were not in agreement with local standards. She thought only intimate couples sleep in the same bed and local people would have a smaller number of people in the same house. She shared her room with another Vietnamese student, and there were three or sometimes four people in her house. Unable to study in her bedroom the living room became her workspace. However, when there was an additional flatmate, it was impossible to study there. The problem became serious when she had an assignment and was desperate for a decent workspace. She resorted to the university library to deal with this issue. 
If there were three people, it would be comfortable. But because there are four of us, it becomes rather cramped. The living room is a bit small. Normally, it's not a problem, but in exam periods, we have to go to the library because there is no place for us at home. If one is cooking or talking on the phone, the others won't be able to study at all. (Bao)

In the same vein, $V y$ shared that there were some kinds of mild tension among people in her flat. It was hard to cater to each other's needs. Despite this, they could manage to tackle this issue when there was a reasonable number of people in the house. However, additional flatmates exacerbated the existing tension.

Some flatmates are noisy, and others are messy. So, there are some conflicts among us. It's ok when there were only three of us. But when there are four or five flatmates, things get even worse. (Vy)

Students need a quiet place to study, but a shared flat can be noisy. Hai usually spent his entire day at the library:

It was noisy. So, when I lived in that house, I usually spent my time studying in the library from morning till late evening. I ate my meals there. (Hai)

Limited living space impacted on everyone in a house, including those who needed a space for personal conversations. Lan was busy working and studying during the day and could only ring her family in Vietnam late at night. However, at this time, her flatmates needed peace and quiet, and even light voices at night might turn into a loud noise:

When it's 12 pm here, it's 6 pm in Vietnam. Yesterday, when I called home at that time, some of my flatmates got irritated. They knocked on my door and asked me to keep my voice down. They said the noise disturbed them. (Lan)

Tam lived with five Vietnamese people. Four of them were students. She shared her room with a female student and her child. She was unclear about how the rent was calculated, but she accepted it. 
We're getting along well with each other. Our house is in a convenient location. It's close to my workplace and campus. So, a shared room or a room of my own is no big deal anymore. If I had a room of my own, I would have to pay more. I don't move out as I'm afraid of the new living environment. (Tam)

Although Tam commented that her house was "always overcrowded", she was generally happy with it and her flatmates. She stayed in the house during her time in Aotearoa-New Zealand. Despite this, she indicated that if she had had a scholarship, she would have got a room of her own and not "share it with any people".

\section{Privacy and personal security}

This theme focuses on how the students' personal security and privacy were affected in their shared flats. Female students were concerned about privacy. Ngoc could not lock her bedroom door because her landlord did not provide a key. She felt "uneasy" about this. It was so "weird" to her, because, in Vietnam, normally, a woman and a man, as tenants, would not live in the same flat. If that happened, the woman could always lock her room door. But in Aotearoa-New Zealand, the landlords did not give their tenants the keys. Her parents in Vietnam were worried about this problem.

Because there was not only me and another woman but also a man in my flat. I was worried. My family was worried knowing that I had a male flatmate. Something could happen if the woman wasn't in the flat with me, and I was alone. Although everyone said that it was very safe here [in Wellington], I still kept my doors locked whenever I was home alone, especially at night. (Ngoc)

The interviews suggested that being unable to have personal security while living with a male flatmate left the student with a sense of anxiety and restlessness. Finally, she managed to lock her bedroom without having assistance from the landlord and letting him know about her concerns.

Thanh's Vietnamese landlady entered Thanh's room when she was not home. Thanh thought this was a lack of respect for her privacy: 
I shared my room with another girl. Everything was OK. I mean she respected [my privacy]. But the landlady, who was Vietnamese, once opened my room door when I was absent, thinking that it was OK to do so. (Thanh)

While Thanh considered it as a lack of respect for her privacy, she did not confront the landlady. She wanted to maintain a good relationship with her. She did not want to be disrespectful by expressing dissatisfaction with the landlady who was older than her. Also, as a tenant, Thanh did not want to make the landlady, who was the authority in the house, upset. Being worried about her privacy negatively impacted on her studies. Later she moved to a new house with flatmates who respected her privacy. She said, "they always close my room door after themselves, and don't use other people's personal stuff."

\section{Issues with flatmates and landlords/landladies}

This theme captures the problems that the students had with the people who shared their houses. Ideally, home is a place for refuge, security and where a person can relax and be themselves. Coupled with the problems resulting from cramped living spaces, threats to privacy and personal security as indicated earlier, problems with flatmates considerably affected the students' sense of not belonging to their houses and contributed to multiple changes in their living situations (see Table 5.1 presented earlier).

Problems may occur between the students and their landlords. At one point, Thanh lived in a house with her landlady - a Vietnamese woman, and the landlady's husband - a Kiwi. The couple had problems, and inexplicably, the husband tried to involve Thanh in the arguments. She thought that he might want to have "an ally". Thanh also had her arguments with the husband. She was so upset with these problems that she could not sleep at night:

I was distressed. I came here to study. I had difficulties with my studies, which was enough for me. Additional problems with him made me feel tired. Sometimes I couldn't sleep and had to ring my friend at night. I had to put on headphones to sleep because they often quarrelled at night. I was very tired. (Thanh) 
Even co-national flatmates can have different priorities and senses of responsibility. As indicated earlier, Vy reported her "noisy" and "messy" flatmates. Similarly, Lan complained that her flatmates left the house in a mess when they were focused on assignments. She had to clean the house and wash the dishes by herself. However, she could not do this all the time because she also needed to work on her assignments.

In assignment seasons, they didn't clean the house. I always finish my assignment first, so I was the one who did it. Recently I had to work and study at the same time, so I was too tired to do the housework. I just washed my dishes. I even didn't cook for the whole week. I don't know if they felt uneasy about that. (Lan)

Lan emphasised that there should be a clear arrangement among flatmates about the responsibility of each person and how to use shared furniture. Reflecting on her experience, she suggested that a cleaning roster could prevent unnecessary problems in the future. She recalled a problem related to a fridge:

We need to agree on how to use the fridge. Sometimes, my flatmates took others' food by mistake, or the fridge was filled with others' food and there was no place for mine. That could lead to huge arguments among us. (Lan)

Lan felt a need to live in a place where she could "get a sound sleep". This implies that she viewed her accommodation in a personal and emotional way. It was not merely a house - a physical building. It should be home - a cosy place where she belonged to, rested, and took sanctuary after a long day outside.

I go to the university in the morning and return home at night. I need a clean and neat living space, where I feel comfortable and can get a sound sleep. (Lan)

Lan shared her room with another Vietnamese student. Although she did not report any problems with her roommate, she felt that it would be best to have a room of her own. She said: "In fact, I want to live by myself in a studio apartment", which comprises a bathroom and a single room functioning as the kitchen, living room, and bedroom. Despite this preference, she stayed in the same house throughout her time in Aotearoa-New Zealand. 
Perhaps the difficulties in finding accommodation when she first arrived were still clear in her mind and she did not want to go through such problems again.

Living with people of various backgrounds and characters may create tensions. For example, Hai's flatmates' lifestyles impacted his life. During holiday times, they would drink beer and wine most days:

[The noise disturbs] my sleep, my life. They were having a holiday. Because they didn't have any homework, they could spend the entire day drinking, even on weekdays. They wouldn't stop drinking until 4 AM. That affected my studies. I was the one who had to accept the consequences of this. (Hai)

Hai chose not to judge his flatmates but work out what he could do to create harmony and persevere with his studies. While with time he became accustomed to the flatmates' behaviour, he eventually moved out.

So far, it has been shown that the housing issue was a multifaceted one, which was related not only to the availability of affordable accommodation but also the students' security and relationships with their flatmates. In the next section, I present how they tackled the problems and managed to create harmonious relationships with others in their house.

\section{Learning to live with others under the same roof}

This theme focuses on the students' negotiations of relationships with their flatmates. The emphasis on creating and maintaining harmonious relationships in Vietnamese culture could be seen in how they responded to differences in lifestyles with their flatmates or roommates. In responding to a question about how to live with people from diverse backgrounds and with different personalities, they emphasised their desire for harmony. They attempted to avoid potential conflicts by controlling their own emotions and behaviours. The focus is on changing themselves rather than their flatmates. This was reflected in Hai's response to his partying flatmates and again in Lan's response to her flatmates neglecting the housework: 
It's easy to move mountains and rivers, but hard to change one's personality [A Vietnamese proverb]. [...] So, I adjust my personality. [In Vietnam], my house was completely my own sky, but now I live in a shared flat. I have to minimise my sky a bit. I don't change my personality, but I control it to fit in with my flatmates. (Lan)

At times, it was hard for the students to maintain their self-esteem and keep their "self" in check. They described a readiness for changing themselves and of caring more about other people's feelings and emotions than their own. Lan used the term "ego" to refer to this process, emphasising that people should not "stick to their egos", and the ideal is to maintain good relationships:

I have a strong character. Sometimes I'm inconsiderate or something. [...] Then I realised I had to be considerate [of other people's needs], not just my own. I had to re-arrange my time and adjust myself to maintain a 'happy' relationship with my flatmates. They have to change themselves, too. I mean, no one should stick to their egos. (Lan)

As presented earlier, Bao was conscious of the age gap between her and her flatmates, which contributed to differences in their lifestyles. For example, she usually went to bed and got up earlier than them. Living with others was associated with self-adjustment. She emphasised that everyone needed to adjust themselves (e.g., everyday routines) so as not to bother one another.

As shown earlier, Hai had problems with his international flatmates' lifestyle (e.g., partying and drinking routines). With time, he became more accustomed to these problems and dealt with them in what could be seen to be a Vietnamese way. He was aware of the tension between his need for quiet to study and their desire to party. Hai positioned his actions as respecting their needs by keeping his own emotions under control.

When I live with people from different countries, cultural differences emerge. Like, I want to learn, but they are drinking, talking, or playing music. [These influence] my learning, my sleep. Some people may lose their temper, pick a quarrel, and have a troubled relationship with their flatmates. I was a hot-tempered person. But here, I 
need to keep my emotions under control. I may let them drink. Like, they always invite me to drink with them, but I go to university to concentrate on my studies. [In that way,] I wasn't influenced. I mean I can have both a quiet space to study and a harmonious relationship. (Hai)

Cultural differences also added to the difficulties. Hai quickly realised that he and his flatmates had different food preferences. However, he wanted to interact with them, so he chose to eat Western dishes with them, although the flavour did not suit his taste. Interestingly, once he realised his flatmates did not like the smell of fish sauce, which is usually used in Vietnamese dishes, he did not cook with it:

We may have different flavour and food preferences. Possibly other people can't eat. Or, you know, when making Vietnamese dishes, we often use fish sauce. But possibly my flatmates can't stand the smell, which may hugely influence them and make them feel uncomfortable. So, in this house, we have to be yielding and tolerant. (Hai)

Hai believed that not making Vietnamese dishes might be a way to make his flatmates feel comfortable in the house. This showed his effort to build a good relationship with them. In this instance and how he responded to their partying routine, he emphasised harmonious relations over his needs.

\section{Communication and Cultural Challenges}

Living in a foreign country which was strikingly different from Vietnam in terms of language and culture posed considerable challenges to the students. These issues significantly affected their interactions with others of diverse cultural backgrounds and could lead to some withdrawn behaviours.

\section{Language difficulties}

When it came to language difficulties, oral communication was often cited as having a vital role. The ability to communicate effectively was associated with the students' capability to perform socially. In response to the questions about difficulties in everyday life, they acknowledged that they lacked practical knowledge about how to start a conversation. 
Trying to make sense of her difficulties with language at her workplace, Ly emphasised that talking to a New Zealander would be different from a Vietnamese person and that she needed to understand how to engage in the conversations. She thought it was important to "see the differences" in terms of conversation conventions and that Vietnamese people "could not communicate with locals in the same way as with the Vietnamese".

Before arriving in Aotearoa-New Zealand, like other TESOL students, Ly thought she could use English effectively in daily life. However, after living in the country, she came to realise that her language ability was not as good as expected.

Before I came here, I thought that I had good command of English. But then, I realised that I needed to learn more about communication. Like, what I say must be suitable in the circumstances. My communication ability isn't as good as I expected. Sometimes, I can't express my idea. I then have to listen to my friends talking to know how to say that. (Ly)

The students had difficulties conversing with local people. They did not know what clues to refer to, which influenced how they felt when receiving a message. One student said she did not know when to close a conversation:

Sometimes, I don't know where to stop. People may be busy, so I shouldn't continue talking. But if I close the conversation, I don't know if I'm impolite or not. I really don't know what sign to refer to. (Bao)

Most of the students had bachelor's degrees in English language, which suggested that they had a solid foundation in the language. Despite this, their responses showed that they had lacked opportunities to communicate in English, especially with native speakers. Reflecting on her language learning in Vietnam, Bao explained that she had extensive knowledge about "academic" but not conversational English. Confronting communication issues left her with a feeling of low self-esteem:

At school, I learned a lot about academic English. When I was thrown into this society, my reaction in daily communication was worse than a kid. (Bao) 
The local accent was difficult for the students to understand. Perhaps, they needed time to make sense of what they heard. It would be useful if people spoke clearly. Besides, the use of slang or colloquial language, which was common in everyday conversations, contributed to the difficulties. In this case, the use of plain English could facilitate their understanding. The students were concerned that the flow of conversations could be affected if they kept asking for repetition. The extracts below from Vy and Hai's interviews support these ideas:

It's hard to understand their accents. Only after they [New Zealanders] talked to each other for a while did I understand what they were talking about. When I talked to them, I had to ask them to repeat a few times. (Vy)

When I talked to them, they used slang that I couldn't understand. Or they told a story, but I couldn't get what they meant, so the conversation was disrupted. (Hai)

Language difficulties threatened the students' social lives by discouraging them from interacting with other people. In the cases of $\mathrm{V} y$ and Hai above, not wanting to disrupt the conversation might result in their silence during the exchanges. Another student, Tam, reported that her social interaction was influenced by her language ability. She had difficulties in getting her message across.

The first barrier was language. I could communicate well in Vietnamese, but not in English. I felt I was like something that shrank. [...] I was afraid that people wouldn't understand me. It affected my speaking and communication. (Tam)

The word 'shrank' implies that she was discouraged from seeking out social interactions, became withdrawn, moved away from communication with other people in English, and back to her Vietnamese networks. In a literal and symbolic sense, it was like her social life was reduced and this was not the life that she used to have in Vietnam.

Language difficulties might have a profound effect on the students' emotions and selfperceptions. One student reported that she felt bad when facing communication problems. She compared herself with other international students and doubted her ability to communicate in English: 
At first, I was a little bewildered and worried because I didn't understand [when people spoke in English]. Some of my friends learned English as a foreign language. I wondered how they could speak English fluently, but I couldn't. (Thanh)

In the following extract, Bao recalled an instance when someone asked her about her academic discipline, and her feeling at that time:

When people asked me 'What's your discipline?' I certainly have to answer that I major in TESOL. Then they asked, 'What does TESOL mean?' I said that it means teaching English. But I felt reluctant because they might wonder how I could teach English when my English was that bad. (Bao)

Nine out of ten students had an academic background in the English language. Although this could be an advantage, it did not guarantee that they would not encounter language difficulties. The association between Bao's academic discipline and her language ability triggered unwanted feelings. She had a sense of low confidence when confronting language issues. Other students - Hai and Tam - also talked about their negative feelings when facing these issues (e.g., feeling uneasy, powerless, and pessimistic):

We didn't understand each other. It was difficult [to have a conversation]. I was not confident that I could communicate as effectively as I expected. [...] I thought that word was simple, but why didn't they understand? So, I felt very uneasy and powerless. (Hai)

Like, we couldn't understand what they said, which made us feel pessimistic and less confident. (Tam)

The students suffered from low self-confidence. Being ashamed to make mistakes when communicating significantly impacted on their confidence. Bao was self-conscious about a possible lack of accuracy and was concerned that she would make mistakes. She remarked that because she was a language student, she paid special attention to the accuracy:

[In Vietnam] I didn't have that feeling, but here I do. [I thought that] my English was not that bad. Having been here for one year, I still make grammatical mistakes and 
feel uncomfortable when speaking. I don't know how students in other disciplines feel, but maybe I'm an English teacher and I always pay attention to mistakes. (Bao)

The students found themselves in a stressful situation that demanded them to have a good command of language to thrive. Their language ability might affect the way other people judged them. Thanh believed that having good language ability was important for a person living in Aotearoa-New Zealand.

Locals may discriminate against and look down on you if your English is bad. I think you should be good at the language or at least can communicate [in English] when living here. (Thanh)

To improve their language ability, many of the students chose to work part-time. Thanh's response was typical among them. She said that interactions with customers and co-workers helped her to improve her language skills.

At university, I just listened to my lecturers or talked to classmates. So, I took a parttime job to improve my English. In the workplace, I needed to actively interact with many people and had more opportunities to communicate. After the first trimester, my language ability improved significantly. I used to be very worried when talking on the phone because I couldn't listen, but now I'm more confident. (Thanh)

During the first trimester, the students encountered language issues in everyday and academic settings. Part-time jobs had a significant impact on their learning experiences. I will discuss these issues again in the next chapter concerning their learning experience. In the next section, I present findings on how cultural differences contributed to their difficulties in everyday settings.

\section{Struggling with cultural differences}

Cultural differences were the students' significant concerns because to a large extent, Vietnamese and Aotearoa-New Zealand cultures were strikingly different. Coupled with challenges in living arrangements and language issues as presented earlier, getting used to a new lifestyle and social norms was strenuous for a number of the students. Thanh shared 
that she needed to be "culturally sensitive" because "sometimes, something is normal for Vietnamese people, but is unacceptable and sensitive for New Zealanders". Although she did not give an example of what she was talking about, this suggested that she was cautious not to make others feel offended because of cultural matters.

The students were conscious of how cultural differences might affect their experiences. As indicated earlier, Hai encountered cultural differences that were related to his flatmates' partying and drinking routines. Bao reported that she once failed to see the priority seating area for the elderly, disabled, and children on the bus and took the seat. Although this was unintentional, she felt "embarrassed". This was due to the difference in seat arrangements for Vietnamese and Aotearoa-New Zealand buses.

I felt really embarrassed. Feeling like everyone on the bus was staring at me. (Bao)

Sometimes, this affected the students' self-esteem. Bao viewed herself as a foreigner in Aotearoa-New Zealand and said that she had limited knowledge about its culture. Throughout more than a year, she faced cultural differences almost every day and had a feeling of "stupidity":

I mean, you weren't born and raised here, at some point, you realise that you don't know anything. You feel very stupid. (Bao)

Challenges might also be related to inadequate understanding of stories or jokes that were related to western cultures. This resulted from limited exposure to the cultures. Missing out on a joke might cause them to feel like not being part of a conversation and made the people they were talking to uncomfortable. The following extracts support this idea:

They usually told a joke but I thought they were serious. I just realised they were joking after a while. (Vy)

Sometimes, they were telling funny stories. Some of them were based on cultures, or movies, or songs that I really didn't know. If I didn't understand, I couldn't laugh. This might turn them off. They might tell a story twice so that I could understand, but I could laugh. That was the problem. (Hai) 
When Ngoc came to Aotearoa-New Zealand, everything was unfamiliar to her. She had to learn from the beginning. Things that she was able to do well in her home country turned out to be difficult, including communication. In the extract below, she attempted to make sense of her experience with cultural differences and cited some situations where she did not know how to communicate properly:

I think my knowledge about communication is insufficient. For example, how to greet each other when meeting? Should we shake hands? If we shake hands upon arrival, should we do that again upon departure? If they don't show that they want to do so, do I shake hands with them? (Ngoc)

The formality in written communication was challenging to some of the students. Bao explained that she struggled with writing emails, especially in determining the degree of formality and the tone to be used. "Sometimes, I'm not sure if my writing style is being too formal or weird. I don't know what degree of informality is acceptable and if I sound rude. That's difficult," Bao said.

Most of the students had limited opportunities to interact or spend time with locals. They were primarily dependent on their co-national network for assistance with daily life and academic issues, and not motivated to extend those networks. They considered this as a major cause for their difficulties in interaction. The following extract from Bao's interview showed the students' typical experience:

I don't live with Kiwis, nor do I have Kiwi friends. I did not have opportunities to interact with them and learn about their lifestyles and thinking. I don't know much about such things. (Bao)

Having encountered and being conscious of cultural issues, the students emphasised that they had to respect cultural differences and learn from each other. They valued healthy relationships among people from different backgrounds. "I met people from different cultural backgrounds with various religious beliefs. No culture is bad. It's important that we respect, learn from each other, and try to understand the differences," Tuan said. 
Together with housing and language difficulties as indicated earlier, the cultural dissonance contributed to the challenges facing the students in the everyday context. Suddenly finding themselves in a country with a different culture affected their interactions. This was associated with their difficulties in forming meaningful relationships with host nationals and these will be discussed in the next chapter.

\section{Chapter Summary}

This chapter has presented the challenges that the Vietnamese students encountered in their living arrangements, communication, and cultural differences. The students were particularly vulnerable when it came to living arrangements. Although having managed to find some potential accommodation when in Vietnam, some struggled to find suitable accommodation in Aotearoa-New Zealand. They ended up staying in a backpacker hostel before being able to find a flat to rent. There was a sense that they lacked knowledge about the rental market. Their co-national flatmates' assistance in daily life issues made their life easier. Losing privileged living conditions in Vietnam presented additional issues to some students. With limited living space, they did not have a decent workspace and went to the university library to learn. Having many flatmates or roommates contributed to the problems (e.g., noise, argument, privacy, and personal security). Despite those issues, they would like to build and maintain a harmonious relationship with their flatmates.

It has been shown that for the students, an ideal house did not just mean a place to live but should make them feel at home. It should be a haven from challenges of the outside world, a place where there are emotional values and where they could "get a sound sleep" and be themselves. However, their current houses did not give them such feelings of comfortability and security.

Language difficulties significantly affected the students' self-confidence and communication with others. Cultural differences resulted in awkwardness in interactions with locals. In the next chapter, I present their experience in an unfamiliar academic environment. 


\title{
Chapter 7 The Quest for Knowledge and the Struggles Along the Journey: Language Difficulties and Demanding Academic Environments
}

\author{
Có công mài sắt có ngày nên kim. \\ If you spend time and effort sharpening and filing a piece of iron, \\ one day it will become a needle. \\ - Vietnamese proverb
}

The students began their studies after managing to secure accommodation. In this chapter, their academic experiences are presented. I focus on two themes: language difficulties in the academic setting and demanding academic environments. The first theme includes two sub-themes: struggles with different accents and struggles with writing assignments and reading materials. The second theme comprises five sub-themes: new teaching and learning styles, heavy workload, struggles with referencing, impacts of part-time jobs on study, and resolving difficulties.

Most students considered language as the biggest challenge. Other issues were related to heavy workloads and negative impacts of part-time jobs on study. The students mainly experienced difficulties with their first assignments and at the beginning of the first trimester. Overall, they felt overwhelmed and bewildered when facing these issues. Over time, many of these concerns diminished, or the students got better at dealing with them.

\section{An Overview of the Academic Context}

In this section, I provide a brief description of the academic setting for the participants, especially the TESOL students. Besides the four courses in Vietnam, TESOL students studied eight courses in Aotearoa-New Zealand, totaling 12 subjects with 180 credits, with two or three assignments for each course. The length of each assignment ranged from 1500 to 3500 words. The students attended ten two-hour classes for each course. Aside from 
working independently, they were encouraged or required, to study cooperatively by participating in-class activities, working in groups, contributing to discussions on the Blackboard, or sharing ideas and resources through email. For each class, they were expected to spend around five hours on self-directed learning. In some subjects, lecturers required them to work in groups of two or three and give presentations in class.

The TESOL students typically had three subjects per week. Before each class, they were required to read a relevant chapter of the set text and one or two other set readings, which were sent to them every week. Reading ahead of classes was expected to give the students background knowledge of the topic. They had time to reflect critically on the implications of the content, to consider their opinions, or to think of possible questions. Most classes required some form of participation, either individually or in groups.

Describing one of his courses, Hai (the Political Science student), said that he was required to write a 1000-word report on the required reading for the week total of around 120 pages. By the end of the course, he could choose the piece with the highest grade.

The number of students in Social Science courses where Tuan studied ranged from 6 to around 20. They were required to attend two to four courses a trimester. Some courses were delivered in the evening. Students were typically required to do three assignments: one individual presentation, one big essay which was 4500 to 5000 words long, and either a short essay which was 1500 to 2000 words long or a journal on what he had read during a week. Group discussions were encouraged in these courses. Two courses were hybrid, which included both traditional face-to-face instruction and online learning.

There was a significant difference in the ratio of Vietnamese students to other students between the TESOL and other classes. In the TESOL classes, about half of the students were Vietnamese, three or four were locals, and the rest were from other Asian countries (e.g., China, Thailand, and Indonesia). In contrast, in Social Sciences classes, there was usually only one Vietnamese student and about half of the students were local ones or from Englishspeaking countries. This was similar to Political Science classes, where there were three or four Vietnamese students, and about half of the students were New Zealanders or from Anglophone or Western countries. 
Having Vietnamese classmates enabled the students to overcome emotional distress (e.g., feeling lost or isolated), and they acknowledged the benefits. However, they did not want to have many co-national classmates because it limited their interactions and connections with local and other international students. Studying overseas, they wished to study in an international environment with students from different countries. Some TESOL students expected to see more local students or more students from other countries in their class.

\section{Language Difficulties in the Academic Setting}

Language played a fundamental role in shaping the students' learning experience. However, language issues are common for international students, especially those who are in their first year and speak English as a foreign language. Most of the students reported difficulties with language, which may be related to writing assignments, understanding different accents, and reading academic texts.

\section{Struggling with different accents}

In Aotearoa-New Zealand universities, the academic staff comes from diverse linguistic and cultural backgrounds. As indicated on the university website, VUW has about half of the teaching staff who were born overseas. At some other universities, the number may be higher. For example, at the University of Otago, over $70 \%$ of academic staff were either born outside the country or achieved their doctorate overseas. This suggests that in many departments of universities, a variety of English accents are used. There is a chance that students will encounter a teacher with accented English.

The students reported on their worries about language issues, explaining that their unfamiliarity with the Aotearoa-New Zealand accent affected their understanding of lectures, especially during their first few months. Some typical quotes include:

I was worried because I was unfamiliar with the accents of people from different countries. (Ngoc)

The New Zealand accent was hard to follow. In that class, my understanding of the lecture was limited. (Hai) 
The students reported that it was difficult for them to "catch" information due to the speaking pace of some lecturers. Thanh, for example, said that she could not understand lectures and catch up with what her lecturers said because they spoke fast.

Although in Vietnam, English is the medium of instruction in many subjects, especially in the TESOL programme, the students still encountered difficulties in understanding lectures due to differences in accents and ways of teaching. Most language textbooks in Vietnam are modelled on either American English or British English. The students typically described what they thought concerning these differences as follows:

I think the Vietnamese are familiar with American or British English, so we expect that we can understand what people say here. But after arriving, we realised that New Zealand English was different from other 'Englishes'. [...] It was difficult for me to understand when people speak English. (Thanh)

I'm not familiar with the New Zealand accent and feel more comfortable with the American accent. Some lecturers speak fast and in a low voice, which is difficult for me to follow. (Hai)

Reflecting on their experiences, the students felt more comfortable with the accent of Vietnamese lecturers and the way those lecturers used English in teaching. Thanh, for example, had difficulties with the accents of Aotearoa-New Zealand academics which were "different from the Vietnamese's accent". Besides, Vietnamese teachers can code switch or use Vietnamese to help students understand difficult points. Usually, when teaching Englishmajor students, teachers in Vietnam may switch to Vietnamese to aid students' understanding of lectures, explain Vietnamese social and cultural issues, or tell a funny story that may be difficult to understand if expressed in English. Lan's interview suggested that while she valued the teachers' ability to explain ideas in Vietnamese, she acknowledged the need to improve her English. English is the medium of instruction in Aotearoa-New Zealand and she could not get her desired results if she failed to do so.

I was familiar with the way Vietnamese lecturers spoke, and sometimes they used Vietnamese to help us understand difficult ideas. The English that we learned in 
Vietnam was different from the English here. I couldn't follow what the lecturer said because they spoke too fast or something like that. [...]. I was worried. If I don't understand lectures, I won't be able to do exercise. (Lan)

Fortunately, after a few classes, Lan came to think that "the lecturer spoke clearly and at a reasonable pace" and that "his accent was easy to understand". She gradually got used to it. Looking back, she was pleased that her learning was not severely affected by this issue.

As indicated earlier, Aotearoa-New Zealand is an ethnically and linguistically diverse country and many academics are originally from overseas and have different accents. Vy had difficulties understanding a lecturer from a non-English speaking country:

It was difficult to communicate with lecturers who weren't English native speakers. One lecturer was from Europe and had a strong accent. I couldn't understand what he said. But I slowly got used to it and found that he was a good teacher. (Vy)

By "good teacher", Vy suggested that the lecturer possessed good teaching skills, was dedicated to teaching, engaged students in learning, and could develop relationships with them. Realising that he was a "good teacher", Vy implied that her limited understanding of lectures was related to her language challenges rather than his accent.

Hai explained that his poor understanding of teaching content inhibited his participation in in-class discussions. His response suggested that language difficulties considerably affected his self-confidence. He did not actively answer lecturers' questions for fear of not correctly understanding them and giving wrong answers:

My understanding of teaching content was lower than my English-speaking classmates. Many times, lecturers raised questions, but I didn't quite understand them, so I might give a wrong answer. (Hai)

Tuan and Thanh expressed similar sentiments. Tuan's understanding of lectures affected his participation in discussions, and Thanh listened to her classmates answering questions instead of trying to answer them herself: 
In the first class, I didn't understand what the teacher said, which affected my interactions with other students [in group discussion]. (Tuan)

I didn't answer [the lecturer's questions]. I just listened to my classmates who were English native speakers. (Thanh)

When the students encountered difficulties in understanding different accents, negative feelings started to emerge. Ngoc recalled that dealing with language issues was difficult, leaving her a sense of self-doubt. She thought she would be unable to use English in academic settings and that she might have an undesired study result due to this problem.

I wasn't familiar with my lecturer's accent. After the first class, I came home and doubted my ability. But I got accustomed to it after a few weeks. (Ngoc)

Ngoc then sought advice from her friend who said "Just take it easy. Gradually you will get used to it". She was comforted and later did not have significant difficulties with accents.

Issues related to language negatively impacted the students' feelings. Thanh, for example, had a sense of "low-value" and described her inability to understand lectures as a "mental shock". Tam had a feeling of passivity when facing difficulties in expressing himself. The following extracts emphasise this point:

I sat there but couldn't understand what the teachers said. I was sad and had a feeling of low self-value. This was like a mental shock. If we fail to overcome it, we can't communicate. (Thanh)

Usually, I couldn't express my idea, so I decided to say nothing. Consequently, I felt that I seemed to become a passive student. (Tam)

Only one student - Bao - reported that she did not encounter significant difficulties with understanding different accents. One reason may be that, in Vietnam, Bao worked for an international company, which gave her opportunities to interact with foreigners. Although she recognised that there were differences between what she learned in school in Vietnam 
with respect to oral communication in English, she could deal with them and did not view them as significant issues.

\section{Struggling with writing assignments and reading materials}

The students experienced anxiety when encountering issues related to completing assignments and understanding materials. Academic reading and writing may pose problems for anyone, including local students, but this was especially the case for international students whose English was not their mother tongue.

Writing assignments was a daunting task for most of the students, especially during their first trimester. After nearly a year, Lan said that she still had "difficulties with academic writing". Hai reported that he encountered issues at the sentence level, and the problem was exacerbated when he was required to write a long assignment.

After I completed a sentence, I read it and found that I didn't write it well and it didn't have any meaning in the paragraph. It was difficult, especially when I had to complete an assignment of 6000 words. (Hai)

Some of the students sought support from the student learning service who check their written work for grammar and clarity. To get their work checked, the students needed to complete their assignments before the deadline, and some did not manage to do this. One reason was that they had to produce several drafts before completing their assignments. Tuan recalled that, for one of his first assignments, he worried because he thought he did not have enough time to complete it. He needed to go through different stages before writing the final version. Tam usually used the service, which she found useful, in the first and second trimesters when she could "complete her assignments in good time".

Reading is especially challenging for the students. Reading issues were complicated because it may be associated with professional knowledge, or language ability, or both. It may relate to their reading skills, that is how they find, link, and make sense of the information that they want. The extract below represents Lan's attempt to make sense of her problem with 
reading academic texts and suggests that the problem was related to a lack of vocabulary and professional knowledge:

I'm concerned about my reading ability. Perhaps I don't have enough knowledge or vocabulary. So, it's really demanding to read journal articles. I may understand all the words, but not the main ideas. I read much but remember or understand little. [...] Sometimes, I'm under pressure when it's time to go to school, but I still don't understand the readings. [...] In class, I can't grasp what lecturers said. After a few classes like that, I was stressed and wanted to give up. (Lan)

Tam and Hai said that they needed much more time than their classmates whose mother tongue was English to complete the required readings. They had to read academic texts slowly and many times to understand and make sense of what they read. The following extracts support this idea:

Other students may complete the reading in two hours, but I may need three or four hours. (Tam)

Because of language issues, comprehending materials was a big problem and timeconsuming. I couldn't understand those materials deeply. (Hai)

Reading materials with a lot of technical terms and abstract concepts presented problems to the students. In this regard, coupled with a lack of vocabulary in English, jargon added to the difficulty. This was related to a lack of professional knowledge. The following extracts from Trung and Lan's interviews showed their experience with those difficulties:

Some materials are difficult for me. There was a lot of jargon and the topic is abstract. I had to read them repeatedly. (Trung)

There are heaps of new vocabulary and technical terms. It was really difficult and took time to understand. (Lan)

In fact, understanding academic texts may pose problems to any student. However, international students may encounter issues that local students do not experience. It was 
challenging for the participants to understand jargon and abstract concepts, not only because those were associated with professional knowledge but also because they were written and explained in a foreign language. This led them to face the double difficulties of professional and linguistic knowledge, which contributed to their unique experience compared to local students.

\section{Struggling with the New Academic Environment}

It was challenging for the students to study in an unfamiliar educational environment. Issues were related to a new teaching and learning style, what they perceived as excessive workloads, and citation and referencing requirements. A number of them worked long hours on top of their full-time studies, which limited their time to study and contributed to their struggles with the new learning environment.

\section{New teaching and learning style}

In the new academic context, the students were unfamiliar with or impressed by aspects of teaching and learning style. These were associated with teachers' respect for students' opinions, thoughtful comments and feedback, learning technology, assignment structures, and requirements.

On the positive side, the differences related to teaching styles between Vietnamese and New Zealand education systems appealed to the students. They typically commented that the lecturers were enthusiastic, respected different opinions, and were friendly:

I was very impressed by a very enthusiastic teacher, who was busy, but always managed to meet us, answer our questions, and give feedback before every assignment. (Tam)

I was impressed by the way the lecturer cared about students. She could remember our names after one or two classes, listened to our ideas, and gave feedback. (Bao)

The students appreciated comments from lecturers on their written work. In Vietnam, inclass tests and exams are popular. Typically, they were notified about the results of the tests 
and received little feedback from lecturers. In Aotearoa-New Zealand, assignments or takehome assignments were favoured and students receive detailed comments from lecturers for sections and the overall written assignment. They enjoyed this difference because thanks to the comments, they knew the strengths and weaknesses of their assignments. The extracts below support this idea:

Lecturers gave thoughtful comments on the strengths and weaknesses of our assignments. (Trung)

They gave feedback on spelling, grammatical, and punctuation errors. I feel that my work was valued. (Lan)

The students commented that their lecturers prepared carefully for classes. Tam described that a lecturer's PowerPoint slides, which were detailed and focused, helped her to follow it easily. Thanh valued clear course outlines that showed what she was going to study, and what she needed to prepare before class:

They provided a clear outline of what we were going to study and expect to achieve. (Thanh)

However, on the downside, some of the differences were difficult for the students to understand. The grading scale used in Aotearoa-New Zealand was new to the students. For example, the university uses the A-E grading system while most Vietnamese universities use the 1 -10 grading scale.

At first, I wasn't familiar with the assessment scale based on A, B, C. I didn't understand how this affected my study result, so I always wanted to have an A. It created great pressure for me. (Tuan)

Another challenge was related to assignments, academic writing, and Blackboard. Although Lan expressed her excitement about the new system, she reported that she was unfamiliar with aspects of the Blackboard. 
In the Orientation, we were informed about many things that we didn't know before. Everything, including the course outline, textbooks, assignment related issues, was available on Blackboard. [...] where I could download many things. I could know what materials I should read before class. (Lan)

Although many students have practical knowledge about technology, having to quickly learn how to use unfamiliar technologies proved a hurdle for them. For example, Hai was unfamiliar with Blackboard, and in the first trimester of study, he had a problem with the system. He thought he submitted an assignment, but his lecturer did not receive it. Fortunately, the lecturer understood and accepted his late submission.

Lecturers were willing to help us. I was a new international student and not sure about the deadline and how things were done here. Perhaps, I didn't know how to submit my assignment, or there were some mistakes in the system that I was unaware of. But after a month, the teacher said that he didn't receive my assignment. I explained the problem and he allowed me to resend it. (Hai)

Bao recalled when she had difficulties linking theory and practice:

I was unclear about how to apply theories to my teaching contexts. Some lecturers explained them clearly and we felt that they were useful. However, some did not clarify them, and we struggled a lot. It was difficult for us to understand the theories. Perhaps they wanted us to reflect critically on those issues. I felt tired and had a headache. (Bao)

The students reported that their assignments were important and took much of their study time. In the comments below, they expressed that they would like to know the assessment criteria or what to include in an assignment. They emphasised the importance of such information. Thanh, for example, was confused because some lecturers did not provide an outline of an assignment:

Of the first three subjects, I was confident that I could do well in two because the lecturers gave a detailed outline of the required content. But for the other subject, 
the lecturer just gave us a topic, with no outline and I was confused. After that subject, I was very concerned about the outlines of assignments. (Thanh)

Lan noted that she struggled to understand the requirements of assignments. Being a student from the twinning programme, she completed the first part of the programme in Vietnam before coming to Aotearoa-New Zealand. Although there were two interrelated parts of the same programme, there were differences in terms of assignment requirements. Lan was conscious of such differences and knew that it was important to understand them. She observed that she and some of her Vietnamese classmates were ill-prepared for assignment-related issues.

When I was in Vietnam, I did some assignments. But I was unhappy because that didn't help me understand or imagine what an assignment in New Zealand would be like. I didn't know what sections should be included. (Lan)

Lan further expanded on this difficulty describing some typical differences and how they negatively impacted on her feelings. The differences were related to the structure of an assignment and the differences in teaching focus between Vietnamese and Aotearoa-New Zealand lecturers (e.g., skills and knowledge). There appeared to be a lack of consistency in the syllabus. Struggling with these, she was worried and began to doubt her abilities. Her concerns diminished after a New Zealand lecturer explained how things were done:

[Before coming here,] we were taught that a research assignment needs to have sections like introduction and literature review. When we came here, we were afraid that we were unable to complete the assignments. But then one of our New Zealand lecturers said that this common template may vary depending on different subjects, course requirements, and what students want to do with their assignments. (Lan)

After completing the first assignment, Lan began to feel more comfortable with doing subsequent assignments. By the end of the first trimester, she better understood course requirements and could deal with them well. 
Although not coming from the twinning programme, Ngoc had similar experiences in understanding assignment requirements. She appeared to be overwhelmed and drowned by a large amount of information that she needed to know. She was also concerned about lecturers' expectations and course information on Blackboard. For a new student like her, these issues appeared to be too much to deal with:

For the first assignment, I was worried because I didn't know how to deal with the requirements of the task. I was unfamiliar with the lecturers' expectations and didn't completely understand the information on Blackboard. The information was simple, but I wanted to see something more specific. (Ngoc)

Ngoc then sought assistance from her Vietnamese friends who came to Aotearoa-New Zealand before her. They gave her advice, which made her more confident in completing future assignments.

Trung said that he was unfamiliar with assignments and that detailed requirements would be useful. A lack of such information could result in significant difficulties for him:

Some lecturers give details of what they expect to see in assignments. We just read those outlines and know what we need to do. [...] Some just give the topic and general requirements rather than an outline. We ourselves have to find out by discussing with our friends or reading materials. (Trung)

Working in groups also posed difficulties for the students. Hai was unfamiliar with group discussions because they are uncommon in Vietnamese universities. Nevertheless, he considered them as opportunities to experience the new learning environment.

I was unfamiliar with the teaching method here. In Vietnam, we're not usually required to work in groups and practical knowledge is not a focus. In New Zealand, group work is encouraged, and we have a chance to give our opinions. (Hai)

Some teaching styles were a concern for a number of the students. Lan, for example, felt overwhelmed and confused, thinking that one of her lecturers "tried to teach a lot". 
I had a feeling that he tried to teach us a lot of things in one lecture. I was confused and overwhelmed. At the end of the class, I didn't remember what he said. (Lan)

Lan explained that the lecturer failed to summarise the main points, and that his lecture did not have a clear focus. Teaching content was not covered in the set readings. She commented that his teaching style contributed to her difficulties in following lectures and her limited understanding of teaching content:

He kept talking without summing up the main points. He talked around rather than about the topic. Perhaps, he loved that way of teaching, but we didn't understand the teaching content. (Lan)

Lan noted that some lecturers required her to "read certain articles before class and talked about different papers in class". She also wondered why the lecturers wanted her "to do certain homework but did not discuss them in class". These left her feeling frustrated.

Although Lan was unhappy about the teaching style of that lecturer, she did not confront him or let him know her thoughts. This could be explained in relation to Vietnamese cultural values in which student and teacher relationships are formal and teaching is a respected profession. A typical response is:

In Vietnam, students are expected to respect their teachers and not to challenge or question them. I have never commented directly with my lecturers because it's disrespectful. (Tuan)

\section{Heavy workloads}

A number of the students experienced difficulties with what they viewed as heavy workloads, which they found hard to keep up with the rest of the class. They linked these issues to problems in writing assignments and understanding the teaching content.

There were differences between Vietnamese and Aotearoa-New Zealand education systems in terms of the amount of required reading before class. According to the students, in Vietnam, teachers and students rely heavily on textbooks and teachers rarely give 
supplementary materials. They stuck to one or two textbooks for tests and exams. Tuan reported that "teachers point out what students are expected to study, and students learn the materials by heart". Looking back on his prior learning experience, Trung emphasised that in Vietnam, textbooks were the main source of knowledge, which restricted his learning to what was presented in these materials:

At my Vietnamese university, I relied on textbooks. [In exams,] I mainly wrote what I read from those but not about things outside textbooks. (Trung)

By contrast, the students were encouraged to complete set readings before class in Aotearoa-New Zealand. Trung was expected to read relevant chapters and journal articles in advance. In class, his lecturers valued students' views and would not "repeat what was included in the set readings but synthesised and analysed them". On the upside, Trung commented that this enabled students to have diverse perspectives and think critically. On the downside, those differences contributed to their difficulties. They were unprepared for the expected "heavy workloads". Although the ones in the twinning programme completed the first part of their programme in Vietnam, they still felt that they were not ready to deal with this difference when they first arrived. One typical response was:

In the first classes, I wasn't accustomed to reading a huge amount of materials. Sometimes, I couldn't complete the required readings before class or not remember what I had read. In Vietnam, I was required to read but not that much. (Ly)

Lan lamented that she had to read an average of 40 to 50 pages for a class, which she described as too much for her.

I was under pressure because of the heavy workloads. I had to read three to four articles for a class, totaling 40 to 50 pages. If lecturers just talked about those articles, it was fine. But if they discussed additional materials, that was too much because I wasn't given enough time to understand. (Lan)

In the following extract, Lan tried to make sense of her learning experience, describing it as overwhelming. She could not complete the required reading and had difficulties with 
understanding lectures due to language issues. She was constantly in a rush, which contributed to her emotional distress.

I had to rush. I felt stressed and overwhelmed because I had to read a lot. I couldn't follow what the lecturer said [because of my listening skill]. [...]. Oh, my goodness, how could I understand and remember what the materials said? (Lan)

Coupled with linguistic issues, the reading requirements were challenging for the students. Hai linked the difficulties in reading with his issues with writing:

Lecturers require students to read a lot. On average, I have to read 120 pages a week, which is a huge workload for me. However, because my English isn't good, it takes me a lot of time to complete such an amount of reading. Then I'm required to write 1000 words based on my readings, which is really difficult. I haven't really understood the topic. (Hai)

Hai further expanded on the difficulties with heavy workloads, describing that he was "tired" and "under pressure" when having to write a long assignment and ensuring that it met the criteria of academic writing - something which appeared to be new to him:

6000-word written work is huge for me. It's hard to write concisely and succinctly and ensure that it shows my content knowledge. Someone may say, "That's fine. I can write up to 8000 or 9000 words." But my written work is often short and well below the word limit. This is my final trimester and there's a huge amount of knowledge and assignments. I'm required to do a project with 15,000 words for around one month. I'm really tired and under pressure. (Hai)

Ly recalled that, in the beginning, she and her friends, who were from the twinning programme were overwhelmed because their teachers required them to read a lot. In the first half of the programme in Vietnam, they were required to read fewer materials. They expected that they would be assigned a large amount of reading, but they were not accustomed to it, which impacted on their learning experience. As the trimester progressed, they became "more comfortable" to complete the required readings: 
We weren't used to it (reading a lot) until we did assignments. We understood why we had to read those articles and felt more comfortable doing so. (Ly)

Having multiple assignments was a source of anxiety for the students. They were constantly worried about the deadlines. Tuan was afraid that he did not have sufficient time to get his assignments completed:

I was unfamiliar [with the educational environment]. I was overwhelmed and worried because there were deadlines. [...] but wouldn't have enough time to complete assignments. There were a lot of things to think through. (Tuan)

The students were conscious of the limited time they had to complete their courses. Lan shared her concerns about this as follows:

Because of my visa status, I need to complete eight subjects in one year. I was shocked because that was a huge amount of knowledge and I didn't have much time. Perhaps I lacked real-life experience, so I couldn't understand all of what I had studied. Maybe I will understand them when I become a teacher in the future. (Lan)

In the extract, Lan tried to make sense of her difficulties, explaining that she needed to complete all the required subjects before her visa expired and that she did not have enough time to digest knowledge. From the interviews with Lan and other participants, it was clear that the issues associated with workloads were prominent in the first few months of their studies and caused them significant distress.

\section{Issues with referencing}

Referencing and plagiarism are two different but related issues which contributed to the originality of written work. While some students had general ideas of referencing, they lacked the knowledge to do it properly, which led to their confusion. They were unfamiliar with how plagiarism was checked at the university (through Turnitin). A lack of understanding or familiarity with these concepts created additional stress for them when submitting their papers. 
Some students had a general idea of what referencing was before studying for their master's degrees. Trung, for example, shared that his teacher introduced referencing in an undergraduate writing course in Vietnam. At that time, he knew that he needed to include information such as the author's name, the title of the text, the year of publication in his written work. Trung commented that what he studied was "superficial" and that other courses did not require him to give references. If he was required to reference, he did it manually or copied references from articles. As a postgraduate student at VUW, he knew how to give proper citations and references.

However, not all of the students were familiar with referencing because of limited education on this issue in Vietnam. Their responses suggested that this was not obligatory and not included in the curriculum at their universities. Introducing this knowledge to students was lecturers' choice and this was inconsistent across universities or faculties within a university in Vietnam. Some students were worried because they had not known how to reference. Hai shared that he was not instructed about referencing as an undergraduate student, which contributed to his confusion. He mixed different referencing styles in one of his assignments:

In my first assignment, I didn't give proper references. My lecturer asked me to use one referencing style. This was the first time I learned about it. These are my weaknesses. [...] After viewing my teacher's comments, I did research online and in the university library to see what referencing style is appropriate for my subject. (Hai)

Similarly, in her first assignment, Vy knew that the university checked the originality of research work but did not understand how it worked. She expressed her concerns:

No one taught me about this before. Only after coming here, did I know how plagiarism was checked. When I submitted my assignment, I knew that it was original. ( $V y)$

While Turnitin, an Internet-based plagiarism detection service, is subscribed to by several universities across the globe, including the university, it is not popular in Vietnamese universities. This contributed to the students' unfamiliarity with the service and might potentially cause distress for them, at least in their first use of the service. Tuan shared that 
in Vietnam, students usually did tests and exams rather than take-home assignments, so they did not need to get their work checked. He was conscious of the importance of plagiarism-related issues in Aotearoa-New Zealand and that Turnitin was a great service that could help him check his written work against the database. However, reflecting on his use of the service, he sometimes felt worried when submitting his work through it. These findings highlighted the need to facilitate students' understanding of issues related to referencing and plagiarism early in their studies.

\section{Impacts of part-time jobs on study}

While employment contributed to the students' funding for living and studying, it could reduce the expected time for their studies. If they failed to organise their time, it might have undesired effects on their academic outcomes. Of the ten students, two - Tuan and Ngoc chose not to be employed throughout their time in Aotearoa-New Zealand. Hai and Ngoc were on scholarships, which possibly explained why they were under less financial pressure than most of their peers. Hai worked part-time in the first few months and then stopped to focus on his studies. Most of the self-funded students had a paid job, except for Tuan. This was because his family supported him financially. Furthermore, it appeared that he was not influenced by peers to have a job because there were no Vietnamese students in his class.

Peer pressure was observable in some participants' responses. Ly, for example, reported that being supported by family, she did not face hard financial times. However, in her interviews, coupled with the desire to be financially independent, peer pressure was explicitly mentioned as a contributing factor:

In the beginning, I was under pressure to get a part-time job because I noted that most [Vietnamese] students who came here before me had a job. Although my family fully supported me financially, I wanted to contribute to my living expenses. (Ly)

Whether or not the students had a scholarship might affect their experience. The selffunded students felt the need to have a part-time job to contribute to their expenses. Although some of them acknowledged the potential negative impact of work on their 
studies, they did not give up working. Undertaking long hours of employment, Tam recalled how tired she was when having no time to rest between work and study:

I worked until 3 p.m., and the class began at 4 p.m. I was worn out. (Tam)

Few students managed to balance their work and studies. Part-time jobs took up their time and impacted on their studies. For some of them, the issues were related to language and familiarising themselves with a new work environment. Ly, for example, worked for a restaurant and found that she lacked vocabulary related to her work. She did not remember the names of the dishes or ingredients, know how to greet customers, or receive their orders. Trung linked his tiredness to issues related to a new work environment. Besides, work subtracted time from his studies:

My work affected my studies. Working here was more tiring than in Vietnam. I needed time to study. (Trung)

Attempting to make sense of the impact of work on his studies, Trung explained that he had difficulties in grasping the teaching content because of being tired after work:

If I spend time working, I won't have ample time to study or refine my written work. [...] I may get exhausted and cannot grasp teaching content. (Trung)

Ly expressed similar sentiments. Her interviews suggested that her studies, including her assignment, were impacted. She sometimes spent the weekends working on tasks that she did not have time to do during the week.

The part-time job affects my assignments. [...] Sometimes I have to spend my weekend working on these or reading books and articles. (Ly)

In the same vein, although Tam recognised that her jobs had a negative impact on her studies and that she had to find a way to deal with the problems, she thought she could manage her time: 
I could manage my time, but not really well. You know, during the first trimester, sometimes I felt sleepy after work. I was tired but still needed to drag myself to class. I had to spend more time on self-study to compensate. That's not good. (Tam)

Most of the students were supported by their families, which enabled them to focus on their studies. In the case of Thanh, she rested assured that her parents supported her. They thought that she should prioritise her studies and were worried when knowing that her work affected her academic results.

At first, I thought I would work to support myself. In trimester one, my study result wasn't really bad, but wasn't good either because it was impacted by my work. Then my parents said that I didn't have to work that much. Once they let me study overseas, they would fully support me. They were right. If I put all my efforts into dealing with financial issues, I couldn't focus on my studies. (Thanh)

The students attempted to seek ways to resolve the impact of their jobs on their studies. Hai recalled that, initially, he was "reactive" in managing his time which implied that he just acted in response to the circumstances rather than controlling it. Then, he came to think that he should prioritise his studies.

My studies may be negatively affected if I fail to balance work and study. [...] In the first trimester, I was just reactive. I used to work two or three days a week which certainly affected the results of my assignments because I didn't have enough time to complete the required readings before class. (Hai)

Hai's scholarship helped him relieve his financial burden and enabled him to focus on his studies. He was motivated to get good study results, so he chose to quit his job.

\section{Resolving difficulties in the academic setting}

The students faced many interrelated problems in the classroom. If they have difficulties following a lecturer's accent, this is likely to affect their understanding of lectures and doing assignments. They employed a range of strategies to handle these issues. Ngoc reported 
that she had difficulties in following what lecturers said; therefore, she usually took the seat at the front so that she could listen to them clearly:

I usually sat with other international students or in the front row to hear more clearly. I wasn't familiar with the lecturer's accent. (Ngoc)

Some students tried different ways to deal with language issues (e.g., recording lectures, reading slides thoroughly, and asking friends for help). Trung and Thanh recalled:

At first, it is difficult for me to understand when people speak English. We recorded lectures to listen again later. But recently, I don't do that anymore because I don't have time to listen at home. So, I just take notes. (Trung)

I couldn't follow my lecturers' accents. I had to read the slides again and again. One of my [Vietnamese] classmates could understand [what the lecturer said] because she used to work in an international company and had a chance to interact with foreigners. I usually asked her to explain what I didn't understand. (Thanh)

Some students said that they felt a need to control the situation rather than just responding to it after it has happened. They remarked that they shift from being reactive to proactive:

During the first two months, I studied without knowing what to do [to enhance my understanding of teaching content]. Then, I slowly got accustomed to the teaching style here, I knew what assignment there would be in a course and what lecturers might require, so I could prepare for it. (Hai)

My overall experiences are positive because I'm active and proactive. When I'm proactive, everything will get better. (Trung)

Peer support was important to the students in the academic setting. There was a sense that it was more convenient and preferable for them to seek help from their classmates than lecturers. They reported situations in which co-national senior students, flatmates, or classmates assisted them in understanding teaching content, navigating the new education 
system, and doing assignments. For example, Tam, usually asked her friends to explain the assignment requirements to her. Hai reported similar sentiments:

Sometimes, the explanation is provided in the article, but I'm still unable to grasp the point. [...]. If I come across an article that is difficult to understand, I may ask my Vietnamese classmates to explain it in Vietnamese. I often talk to them about how they do assignments and research on an issue. They studied here longer than me, so their English is better. (Hai)

Other students relied on lecturers' instructions and feedback for previous assignments to improve their following work. This is consistent with what I discussed before on the significance of lecturers' comments on students' assignments. Lan explained that the comments helped her see not only her strengths and weaknesses but also her lecturers' expectations about her work:

Here, we have detailed instructions, clear requirements, and feedback on every assignment. Based on the feedback, I can do better on the subsequent assignments. [...] For the first assignment, I was concerned about whether I understood the lecturers' feedback and what they said correctly. I had no idea what their expectations were and how things were done here. I just followed the instructions, and the results were good. (Lan)

I practice more and more. I can draw lessons from lecturers' feedback and improve subsequent assignments. (Tuan)

Besides emphasising the role of lecturers' feedback in students' revision and improvement of written work, the students relied on assessment criteria as outlined in the course syllabus to figure out how to proceed with their assignments:

Lecturers give criteria for assessment which we can rely on to do our assignments. (Trung)

Being conscious of their difficulties in understanding the teaching content and reading, the students spent more time on self-directed learning and viewed it as an important 
supplement to classroom-based learning. Tam shared that she took initiative in self-studying to enhance her understanding of assignments and set readings:

During the first two trimesters, especially when I had assignments, I almost spent my entire day at university and just came home to sleep. My reading pace is slow, so I just kept reading. (Tam)

\section{Chapter Summary}

This chapter has presented the learning experiences of the Vietnamese students in Aotearoa-New Zealand. It demonstrates that their experiences were largely associated with differences between two different educational systems. The students were overwhelmed and bewildered by the new educational environment. Different accents significantly contributed to their language difficulties. Struggles with writing assignments and reading academic texts were observable, but after a few months of completing the first assignment, these issues did not impact them much. Although the TESOL students had completed the first part of their programme in Vietnam, they still encountered difficulties when doing the second part. Unfamiliar teaching and learning styles were both enjoyable and challenging, and there were concerns about understanding assignments' requirements. Heavy workload contributed to their difficulties in keeping up with their studies and understanding lectures. They encountered problems with referencing, but most understood how to give proper citations and references after the first or second assignments. As indicated in the previous chapter, part-time work was beneficial to them. Despite that, in this chapter, it has been proved that part-time work negatively affected them by subtracting their study time. Peer support was important as it contributed to their ability to deal with difficulties in academic settings. In the next chapter, I will present the students' experiences concerning their struggles with home-related feelings, issues in forming meaningful relationships, and their transformation process. 


\title{
Chapter 8 | Learning to Live on One's Own: Dislocation from Home and Friendship Networks
}

\author{
Đi cho biết đó biết đây, \\ ở nhà với me biết ngày nào khôn. \\ Travelling brings you knowledge,
}

Staying home with your Mom does not make you wiser.

- Vietnamese folk song

...and here was the end of the journeys.

Being away from home to study was challenging but transformative. The focus of this chapter is on the students' feelings about home, networks, and struggles to become independent. This chapter includes three themes: Struggling with home-related feelings, barriers and enablers in forming a meaningful relationship, and learning to live on one's own. The first theme consists of two sub-themes: homesickness and yearnings for connections with families and friends, and family - concerns and motivation. The second theme includes two sub-themes: enablers in forming relationships with co-nationals and other international students, and barriers in forming relationships with host-national students. The final theme comprises five sub-themes: lack of preparation for a new life, struggling to survive, becoming an independent person, the ability to take care of one's self, and financial independence. The theme 'lack of preparation for a new life' was included in the paper mentioned in the previous chapter, entitled: 'The experiences of Vietnamese students in New Zealand: A new country - a new home'. Finally, I capture the essence of the students' experiences.

Most of the students felt homesick at some point during their time and expressed a desire for connection with friends and family in Vietnam. They were concerned for their families who they had left behind. Most of them were ill-prepared for their new lives. However, living in the country forced them to become independent. They learnt to take proper care of 
themselves in terms of basic daily needs (e.g., eating) and unique needs (e.g., health and time management). Through being employed and contributing to living costs and tuition fees, most became more financially independent.

\section{Struggling with Dislocation from Home and Familiar People}

This theme deals with the students' emotional distress triggered by being away from home. Most of them expressed feelings of homesickness and worried about their families. For a number of them, these significantly affected their living experiences and were also associated with health problems.

\section{Homesickness and yearning for connections with families and friends}

This theme describes how intense the students' feeling of longing was for connection with families and friends in Vietnam. Being away from home caused high levels of emotional distress among some of the students. This impacted on their daily and academic lives (e.g., issues with housing and assignment pressure). They reported being distracted from their studies and feeling guilty about leaving their families. Although the students had a range of concerns about their families, what commonly emerged from their responses was that these feelings were related to a sense of not belonging in Aotearoa-New Zealand.

The students were excited when they first arrived and looked forward to their new lives as students. However, at some point, they each experienced homesickness. They struggled to find affordable accommodation and negotiating relationships with their flatmates was also a source of worry. Some of them changed accommodation a few times. Not having a stable place to live made Vy miss her cosy house in Vietnam. She recalled:

I was really homesick when I changed accommodation. That was the time when I wanted to go home. (Vy)

Technology helped the students to deal with the times when they missed their families. Some of them talked with their families using calling apps on their phones. Thanh reported that talking to her parents helped her cope with this feeling: 
My mother rings me every day. My parents know that I need to focus on my studies, so they don't want me to call them. At times, I call them first when I miss them so much. (Thanh)

In the case of Tuan, his parents were not at home on weekdays due to their work commitments. He could talk to them only on weekends, usually on Sunday. Focusing on studies and thinking about his return to Vietnam helped reduce his homesickness:

I tried to bury myself in studies to forget that feeling [of homesickness] and reminding myself about the reason why I'm here. (Tuan)

There were different levels of homesickness. For some students, this feeling came and went easily, and did not have a significant impact:

I feel homesick when I'm not busy with my studies. But when I have to give all my attention to them, I don't have such feelings anymore. (Tam)

For the others, the negative impact of being away from home on their emotions was observable. In the extract below, one student tried to make sense of her homesickness, reporting that it was associated with emotional and psychological distress (e.g., sadness and depression). There was a time when she felt down and was tearful amid a conversation with her friends. These feelings came up unexpectedly for no apparent reason:

When I finished my assignments and had some free time, I felt homesick. Sometimes, I didn't know why I was sad. Once, I was with my flatmates and tears formed in my eyes. They didn't understand why. (Ngoc)

Ngoc did not consider homesickness as a good reason to call her family. She used Google to see where her house was and realised that she was thousands of miles away from home:

Feeling homesick, I sometimes even got on Google Maps to find the way home. At that time, I didn't call home because I wasn't a person who often talks about problems with family members. I was very reluctant to do so. (Ngoc) 
Living away from their loved ones and encountering emotional pressures, the students came to recognise the importance of talking with someone who listened without judgement and understood them at a deep level. Ngoc expressed the need to have such a friend explicitly. Her flatmates were helpful, but as international students, they had their commitments and sometimes did not have time for her.

I want to have a friend whom I can talk to and who understands me. I have very friendly [Vietnamese] flatmates. However, I can't share much [about my issues] with them because they're very busy. [...] I think it's necessary to have a person who can understand us. ( $\mathrm{NgOC})$

Homesickness was not just about missing one's home but also about missing family members. Bao missed her young children and was conscious that this was the period that they most needed her care and support. She sacrificed meeting these needs for her overseas studies. This was a source of distress that affected her studies and health. She emphasised that she was under pressure to study well. With this sense of achievement, she thought her sacrifice would be worth it:

I think living far away from home and the way I give myself pressure lead to more difficulties in my studies. For the first and second assignments, I felt so stressed that I couldn't think of anything, which didn't result from the assignment but me. This was because I thought I had everything I needed for my studies. I came here, leaving my family behind, and spending a lot of money. If I didn't get a good result, it was my fault. (Bao)

Apart from missing her children, Bao missed what was familiar to her. After years of living and working in Vietnam, she had established deep relationships with people around her. Her homesickness was also associated with a larger sense of loss relating to her social relationships. She shared that when going out on the streets, she "had the feeling of strangers" around her:

When I go out by myself [in Vietnam], I don't have the feeling of strangers around me. In Vietnam, at my age, I have friends, colleagues, family, and many other things. 
We love each other a lot. But here I'm alone. Although I have friends and classmates and we love each other, I still feel that something is missing. (Bao)

Spending time with her Vietnamese friends helped her deal with homesickness. Making sense of her experience, she used a vivid expression: "feel loneliness in crowds". She needed a companion in these unfamiliar surroundings. However, her friends just helped her temporarily forget her loneliness:

I still feel something unfamiliar to me. If I go to a movie, I will ask my flatmates to go with me. I don't like going out by myself because it makes me feel lonely in crowds. Whenever I open my eyes, I just see strangers around. (Bao)

The extracts above show that Bao did not have a sense of belonging in Aotearoa-New Zealand. Her feelings were related to her lack of meaningful connections with other people. This was mainly about the quality of relationships rather than the quantity. She indicated that there was an age gap between her and her classmates, which contributed to her difficulties in forming significant relationships. Apart from this, the separation from her children played an important part in her sense of not belonging.

\section{Family - concerns and motivations}

The students' feelings and thoughts about their family members who were in Vietnam were twofold - they were worried about their families and considered these people to be an important motive for them to overcome difficulties and study well.

In the case of Bao, her children were still one of her major concerns although her husband and other family members helped to take care of them. Before coming to Aotearoa-New Zealand, she had never expected that the difficulties of being separated from her children would be so challenging:

My major problems are related to beloved people left behind. I separate from my children. I knew that it would be difficult, but after I came here, it was perhaps five or six times as difficult as I expected. (Bao) 
Prior to her departure, Bao thought that one year would pass very quickly. However, she realised that leaving her children in Vietnam made her year seem much longer. She was conscious of the distance from them and the time she would be away. These problems were often overwhelming and made her doubt her decision to study overseas.

I didn't know if my decision to study abroad was right or not. [...] If my studies weren't going on well, I would feel very bad. It was just my emotion that caused me to feel like that. At that time, I thought I was kind of under much pressure resulting in ridiculous thinking in my head. (Bao)

Bao shared that when she faced these issues, negative feelings started to emerge. She vividly described that she felt "empty", "missing something", and "left her part in Vietnam". She perceived her children as an inseparable part of herself and being separated from them would be painful to her:

I thought it would be fine, just one year, not a very long time. Before I left, I didn't have much time to prepare and couldn't take my two children with me. I always feel empty or missing something. I came here but still left my part in Vietnam. That is my feeling. Then I felt one year is so long. I'm counting down to the day I go back. (Bao)

Lan was worried about her parents and family, who were very important to her. She said that her father often worked on the night shift and was home on Saturday only. [...] The difference in time zones limited her choice of time to call her parents. She could only call home at $6 \mathrm{pm}$, which would be midnight in Vietnam:

I felt unrest when I couldn't help my parents take care of our house. My home is very important to me. I can leave everything behind to go home if necessary. [...] Now that I'm here, I can't do anything for my family. I don't know what's happening at home. [...] I want to take care of my family. I want my house to be cosy. I don't want it to be cold. (Lan) 
There were four people in Lan's family: Lan, her sister, and her parents, but her sister was usually away for her business. She was worried about her parents' health. If they got sick, no one would take care of them.

My parents have two daughters, but my sister and I aren't home to take care of the house and the family. My family is very important to me. My father is nearly 60 years old. All my success and qualification will have no meaning if he gets sick. [...] I can stay here or return to Vietnam and get a good job with my qualification. My thinking is simple. I have studied a lot, now I get a good job, make my father a meal every day, and clean the house. So, I'm considering returning to my country. (Lan)

Lan viewed her parents as the driver behind her determination to get her desired academic outcome.

I have to overcome every difficulty. [...] My family gives me opportunities and expects me to obtain a good qualification. They always think for me and support my decision. People with a university qualification might move on to employment. But for my family, it's ok to obtain further education. If I have good academic achievement, they will be very happy. That is my motivation now. (Lan)

Being concerned about their children and parents played a fundamental part in Bao and Lan's decision to return to Vietnam. There was a sense that they would not have peace of mind if they could not reunite with their families after graduation.

\section{Enablers and Barriers in Forming a Meaningful Friendship}

As international students, the Vietnamese students met and connected with a wide variety of people from diverse cultures and backgrounds. Making friends was facilitated or hindered by various factors. Friendship was important for personal and emotional needs. They wanted to have someone who could understand them, give advice, help them out with issues, and alleviate emotional distress. These desires helped draw the Vietnamese students together. One of the best ways to build mutual understanding is through interactions. A lack 
of interactions between the Vietnamese and local students contributed to the difficulties in building meaningful relationships.

\section{Enablers in forming relationships with co-nationals and other Asian international students}

Vietnamese friends. Leaving their families and friends behind, the students felt a need to establish and rely on a new network. Their primary network consisted of Vietnamese classmates because these students were the ones with whom they interacted the most. Their similar backgrounds in terms of culture, education, expectations, and difficulties helped them understand each other and assist with emotional issues. These co-national networks could offer emotional support and help to relieve stress. Having same-culture friends has a positive impact on their experience in both daily life and studies. They needed to have someone to talk to or turn to when they encountered difficulties. Having a conational friend helped reduce the feeling of isolation or homesickness. As people who live away from families, the students could provide solace to each other.

Students with access to clearly defined co-national groups might view their co-national friends as an important source of empathy and refuge. The extract below from Bao's interview supports this point. Unlike her family in Vietnam who could not understand her problems, her Vietnamese flatmates, who were also her classmates, understood what she experienced in university and daily life and could assist when she needed:

If I need someone to talk to, I have two Vietnamese flatmates, who are also my classmates. They can understand my experience and may have similar experiences. It would be easier to talk to them. My family wouldn't understand these. (Bao)

During the first few days, some students felt some kinds of distress. Initially, Thanh felt isolated and lost. She perceived differences in personality with her classmates, which made it difficult for her to build a relationship with them. Thanh did not expect that she could establish a meaningful relationship with her Vietnamese classmates. In Vietnam, they were not close friends, but being together through a hard time helped the relationships grow. They were living together, and her friends helped her when she was ill. A sense of 
reassurance and peace of mind contributed to her strong bond with them. At that time, she had assignments and was under study pressure.

The more friends we have, the warmer we feel. I'm more attached to people who live with me. I got to know some of my friends when we were studying in this programme in Vietnam. In class, we just said 'hello' or talked about our studies. But here we live together and help each other. I was once hospitalised and they took care of me, just like a family. I have never expected... Why were they so kind to me? (Thanh)

Co-national network provided the students with a feeling of cultural identity. Trung explained that having many things in common, including "the Vietnamese spirits" contributed to his ability to build a network with Vietnamese people:

I have a closer relationship with Vietnamese people here. We share Vietnamese spirits, language, culture, and similar viewpoints. Vietnamese people are willing to help each other and be close-knit. So, it's easier to form a friendship with them than those from other countries. (Trung)

Coupled with cultural identity, a sense of comfort and understanding was a significant factor in Trung's relationships with others in his co-national social circle. He felt comfortable with his Vietnamese friends and hence was not motivated to have friends from host nationals.

Asian International students. As Asian students in a foreign country, the participants felt links with other international students. All reported that it was easier for them to establish meaningful relationships with Asian international students than local ones. Other international students were viewed as more open to friendships than some local students. They were conscious of the importance of having shared interests, beliefs or opinions in relationships and acknowledged that having a common ground could significantly contribute to the bonding amongst people. Lan explained that she and other international students shared common experiences and feelings. Living and studying in a foreign country brought them closer together and caused them to be open to new relationships. 
I have friends who are my classmates from China and Thailand. It's easier to establish a relationship with and talk to them than local students. It may be because we're international students and we share some feelings. For example, they also live far away from their countries and feel homesick. (Lan)

The students felt that it was easier to make friends with Asian co-workers than with local ones. Vy worked with both Asian and local people in a restaurant. She found it easy to socialise with Asian co-workers because she could talk with them comfortably. She emphasised that she felt like "an outsider" or "the odd one out" among the Kiwi co-workers. She explained communication issues hindered the development of their relationships:

When I talked to my Kiwi co-workers, I continuously asked them to repeat what they said, which might make them feel uneasy, and not want to talk to me. [...] I felt a bit uneasy. I was like the odd one out. But I couldn't do anything about it. I cannot have a strong relationship with them because of language barriers. (Vy)

Having opportunities to study and spend time together developed their relationships. Bao explained that because she studied with many Asian students in courses, it was easy for her to make friends with them. Similarly, Lan had friends who were from China and Thailand. Trying to make sense of her feelings, she commented that sharing some common features of Asian people caused Lan to feel links to Asian international students or feel that she had known them before.

Most of my friends are from Asian countries. Just seeing they have the same hair and eye colours makes me feel close to them. (Lan)

Lan felt that other international students were not different from her Vietnamese friends. In a foreign country, these feelings considerably contributed to their relationships.

\section{Barriers in forming relationships with host-national students}

This theme focuses on the students' ability to form meaningful relationships and within the theme, influences of language issues and a lack of common ground are highlighted. All the students acknowledged the benefits of having friends who were local students. However, 
most of them noted that there were potential difficulties in forming meaningful relationships with them. They said that local students had much to offer to international students and valued the diverse perspectives that local students brought to conversations about academic and non-academic issues. Although these enriched the participants' experience, it took more than that to build meaningful relationships. "It's good to have friends with different perspectives, but it's difficult to establish relationships", Lan said.

The small number of local students in the class could be another cause for the difficulties in making friends. There were few local students in the twinning programme, and, except for the Vietnamese students who accounted for half of the class population, the rest was mainly from Asia. Bao said that the local students in her programme usually chose online courses:

There are few Kiwis in our class, but we rarely see them because they usually choose online courses. [...] There's a young local student in the class, but he does not talk with anyone. (Bao)

The age gap was another possible contributing factor. Different generations might have different attitudes or opinions, which affected the students' interactions with their classmates of different ages. Friends of a similar age could understand each other well. Having classmates who were much older than him, Tuan commented that the age gap significantly impacted the possibility of forming relationships:

I don't have friends [who are local students] because most of my classmates are much older than me and are the same age as my uncles and aunts. In my first class, there were thirty students, but only three or four students were of my age. (Tuan)

Similarly, in an attempt to make sense of the possible issues around the age gap, Bao explained that middle-aged students might be too busy to establish new relationships:

They're in their middle-age and perhaps they have their own business, personal commitment and network. They're busy with their lives and don't feel a need to make friends with us. (Bao) 
Most of the students perceived a lack of common ground with local students. For example, Tuan felt that he could "hardly find a common ground" with them. Lan's response suggested that local students would have different experiences, which would make it difficult for them to understand international students' experiences. This affected the flow and the types of conversations that they could engage in, which caused distance in their relationships:

There were one or two local students in my class. International and local students also had their own concerns. Perhaps, I felt a distance between us, so I didn't form a relationship with them. We did have some small talks but did not communicate much. We were not friends indeed. It wasn't because they didn't talk to us but like we didn't have anything in common. (Lan)

For the students, being able to engage in conversations around personal issues indicated a trusting relationship. This was related to language issues and cultural norms on how to break the ice. Tam found it difficult to build a deep and meaningful relationship with superficial conversations about common daily life issues.

I don't have many close friends here. In class, I mainly talk about study issues with my international friends. Outside the classroom, we just have small talks. (Tam)

Although the internet helped the students to connect with many people, they made a distinction between online and real-life communication. They valued the latter and thought it was effective for developing relationships. Similarly, Thanh emphasised the crucial role of understanding in relationships and asserted that online interactions through social networking sites (e.g., Facebook or Twitter) were not enough to form a relationship:

I mean, if we just talk in class or communicate through social media... we cannot really show our feelings. With face-to-face communication, we will have a better mutual understanding. (Thanh)

Language barriers might impact the students' ability to socialise. This might be one of the possible reasons for some students' preference to make friends with co-national people and reluctance to communicate with other students in English. Tam's response reflected a 
common situation that most of them experienced. In making sense of her experience, she described it as social withdrawal:

Perhaps language is a barrier. I can communicate well in Vietnamese, but not in English. I felt I became withdrawn. (Tam)

Differences in cultures and lifestyles might cause interaction issues, which affected how people feel about each other and made sense of others' behaviours. Vy felt that everyone had problems. They had little time for and were not open to each other.

I feel that it's hard to live here. I don't know. People aren't open to each other, which is different than those in Vietnam. [...] While we're friends, we have to deal with our problems by ourselves. (Vy)

There was a sense that the students lacked the motivation to form long-term relationships because they thought they would not remain in Aotearoa-New Zealand long enough to do so. Most wanted to return to Vietnam after graduation; therefore, they thought it would be difficult for them to maintain relationships with local students or those from other countries. Geographical distance served as a potential barrier. Although they expressed a desire to build relationships, these issues discouraged them. The following extracts illustrate the students' viewpoints:

I attended a class where there were many students. Local students were friendly. But once the course ended, everything did too. (Lan)

I just stay here for a short time. So, I have no need to establish long-term relationships. (Tuan)

We need a longer time to build meaningful relationships. (Trung)

It was challenging for the students to make friends at university and in their workplaces. Most of them had Vietnamese co-workers and their workplaces appeared not to be a place to make friends with local co-workers or those from other countries. For example, Trung said that, at his workplace, building a relationship with a Vietnamese person was easier than 
someone from other countries. He noted that he just stayed in Aotearoa-New Zealand for a short time and did not wish to form long-lasting relationships.

Most of my co-workers are Vietnamese. It's easier for us to become friends or start a conversation because we share a language. [...] My casual acquaintances are my coworkers or someone I know in my daily life. We just keep contact for a particular period of time rather than for a long time. For example, when we are still working in the same shop, restaurant, or studying in the same class. After that, I may not see them again and don't contact them anymore. (Trung)

Most of the students emphasised that they needed to spend time on their studies rather than expanding their networks and that successful fulfilment of degree requirements was their priority. This was associated with their struggles in academic settings (e.g., heavy workload, difficulties with reading academic texts and writing assignments, and unfamiliar academic environment), which were discussed in the previous chapter. Because of their priority for the demands of course work and other academic issues, the students had little time to engage in other activities, including forming new friendships. While Lan was conscious of the academic challenges and wanted to complete her studies within the expected timeframe, Ly said that her job was her second priority, after study.

I want to focus on my studies, but I fear that I cannot meet academic demands. Our university has high academic standards, so I need to be self-disciplined. I am under heavy workloads. (Lan)

I want to focus on my studies and my job. I want to be by myself and not spend time hanging out with friends. (Ly)

\section{Learning to Live on One's Own}

For international students in general, overseas study is unique and means more than just learning and living experiences. All the Vietnamese students described their experiences as life-changing and enriching ones. As international students, they went through situations 
that they would not experience in Vietnam or when living with their families. Reflecting on challenges facing her younger friends, Bao suggested:

Students who are living with their families in Vietnam need to be prepared to become self-reliant. They should know how to cook, do the washing, and shop. Otherwise, they will be shocked when living in New Zealand. Once they step out of their families' care and comfortability, [...] they may have a tough time settling into their new lives.

Living and studying in a foreign country with unfamiliar educational and everyday environments forced the students to transform themselves to overcome difficulties. This provides them with opportunities to construct their identities and become better versions of themselves. Overseas study helped them to become more mature and confident and be able to live on their own.

\section{Lack of preparation for a new life}

In the students' stories, there was a sense of not being aware of how life in a new country would be different. When asked about how they prepared for their new lives, they reported that they started their travels hastily and had not thought about the difficulties they might encounter or the differences between life in Vietnam and Aotearoa-New Zealand. Most of them did not have any concrete plans but decided on what to do based on how things turned out. Ly reported that she did not prepare much and that she "just played it by ear". Hai was unaware of geographical differences between Wellington and his hometown, which he soon realised and experienced:

Initially, I just thought New Zealand was like Ha Noi. It (Ha Noi) is a plain. I didn't think it's a mountainous region like this. When I first came here, I lived in a rental flat that was not really near the university. I walked to university and I was exhausted. Our university is on the top of the hill. Going up the hill was tiring. After that, I took the bus and I started to feel better. (Hai)

Hai's hometown was Hanoi, the capital city of Vietnam. Wellington is built on hills and sits on a natural harbour while Hanoi is nearly 56 miles away from the coastal area and includes 
three regions: the Red River Delta, the midland area and mountainous zone. Hai lived in the delta area which is the most populous. This was why he thought about Hanoi as a large area of flat land.

The students prepared themselves for study-related issues and neglected potential daily living issues:

I have never thought about that. I only thought that I came here to study. I would have international friends, and lecturers, and do assignments. That's all. (Thanh)

It is like when I prepared to study abroad, I was just concerned about how teaching and learning styles [in New Zealand] were different. I didn't think much. (Lan)

Initially, the students' lack of preparedness resulted in their feeling shocked and unable to cope with problems. They confronted everyday living challenges while struggling to adjust to the new academic setting. Being poorly prepared for lives in Aotearoa-New Zealand contributed to the issues concerning living arrangements discussed in the previous chapter and the following sub-theme.

\section{Struggling to survive}

In Vietnam, most of the students were middle-class professionals, who lived with their parents. They were strongly tied up with their families and had limited experience of living away. Most found themselves in a stressful condition when separated from their families. The extended family was their main source of support. In coming to Aotearoa-New Zealand, they lost the comfort and security of what had been their everyday support systems. Looking back, they recognised they had led privileged lives, and some of them struggled when they had to learn to live independently.

The students found living in a new environment overwhelming because everything was unfamiliar to them. There was a sense of uncertainty about how they could proceed with their lives and studies. Ngoc, for example, shared that she had to grapple with several concerns when she first arrived: 
In the beginning, I couldn't imagine how my life would be going on. I didn't know how to make new friends, deal with changes, keep regular contact with family and friends in Vietnam, get good study results, and balance my studies with other matters in daily life. (Ngoc)

The practical skills that the students have in managing everyday tasks and their capacity to develop those skills strongly influenced their living experiences. Thanh was aware that she lacked the necessary skills (such as cooking) to lead an independent life. She "lived like a princess" in Vietnam. Thanh arrived in Aotearoa-New Zealand with her four Vietnamese classmates, whom she hoped to live with. They stayed in a hostel for the first two weeks until Thanh decided to leave because they could not find a place big enough to accommodate all five. Suddenly, she had to enter life for which she was not ready. During the first few weeks, she ate boiled eggs and vegetables and lost weight. Thanh recalled how she felt during the first few months:

I have all these feelings. When I lived away from my family, I had to do it all through by myself. I was afraid, really afraid. I was worried that I wasn't able to get through [this difficult period]. (Thanh)

Problems facing the students were related to the inability to meet daily living needs, for example, some of them were not good cooks. Thanh reported that when she was in Vietnam, she thought that she could take care of her health. However, after coming to Aotearoa-New Zealand, she came to realise that she was wrong because she could not cook herself a healthy and balanced diet.

I wanted to eat that kind of food but didn't know where to buy it. Then I just kept eating 'Western dishes'. There was a time when I only ate Sushi. Now I'm sick of just seeing Sushi and couldn't eat other dishes. Then I realised that what I was doing wasn't the right thing for me. I thought I needed to return to Vietnam, or I might die here. (Thanh) 
Failing to prepare decent meals greatly affected Thanh's health and losing weight was a clear indicator. This showed how hard it might be for the students who were inadequately prepared for an independent life.

I had so many troubles with my diet, and my health was heavily influenced. When I first came here, I couldn't adjust to the weather and didn't know what to eat. I often came to Student Health. [...] I lost some weight. When I was in Vietnam, I weighed 45 kgs, but now, I weigh 43 kgs. (Thanh)

In Vietnam, Thanh had a good job and a supportive family. In the interviews, she indicated that she had a sense of status loss when doing manual jobs that did not allow her to utilise her professional knowledge. This left her with a feeling of low self-esteem and depression. Leaving her family when she was inadequately prepared negatively affected her living experience and contributed to her decision to return home after completing her degree.

In the following extract, Bao tried to make sense of her living experience by contrasting her life in Vietnam and Aotearoa-New Zealand. She emphasised that the absence of her familiar network affected her living experience and caused her to feel insecure:

It's true that when I'm away from home, I have a feeling of insecurity. [...] Like, in Vietnam, we had friends and family, but in New Zealand, we have [Vietnamese] friends who are studying in the same programme, but they're not really close friends like the ones that I had in Vietnam. (Bao)

In Vietnam, Bao had a good job, the support of her extended family, her children, and close friends. She had a sense of insecurity when leaving her family and familiar friendship network. Bao expressed a desire to return to Vietnam after finishing her studies.

\section{Becoming an independent person}

Moving to Aotearoa-New Zealand exposed the students to difficulties. However, they valued their experiences, both positive and negative, and were aware that overcoming these challenges helped them grow as a person. Living away from family, they had to be able to cope with issues and learn to take care of themselves. Some described this as a journey to 
get out of the comfort zone, live their own life, or continue the journey of "getting out of the village's bamboo range".

After living in Aotearoa-New Zealand for a few months, they learnt more about themselves. They reported that while trying to handle challenging situations, they ended up realising that they had been mistaken about themselves. Things turned out to differ from their expectations. For example, when Thanh was in Vietnam, she thought she could deal with everything in the new living environment by herself. She felt like she had lost her "solid rearguard", someone who could protect her.

When I came here, I lost my 'solid rear-guard'. I didn't know what to do. That's when I saw the differences [between my expectations and experiences]. Also, when I was in Vietnam, I thought my life would be easy here. But it wasn't at all. (Thanh)

Stepping out of their comfort zones and putting themselves in uncomfortable and difficult situations, the students recognised what they did not think about before: their fear. They were uncertain about what was waiting outside. Thanh felt fear and anxiety when living away from her family.

When I got out of my family and didn't have my parents around. I had to do everything by myself. I really feared it. I was afraid that I couldn't overcome [difficulties]. (Thanh)

Outside of her home was where the transformation happened. Without her parents, Thanh had to learn to cope with issues. She knew that she had to be responsible for herself and be self-reliant. Most of all, she conquered her fear. In the following extract, she described how she had changed:

I was proud of myself. [...] I think I need to help myself. If I couldn't manage by myself, I would ask other people for help. [...] I don't fear anymore. When I encounter any problems, I stay calm, sit down, and find a solution, or ask other people for help. If I fear, I will get confused, and won't know what to do. (Thanh)

Within six months, Thanh had settled in and felt more comfortable with her new life. She was able to solve problems on her own: 
After 6 months, I felt that I could do things on my own. Some people asked me if I was sad when being alone, I said "No". I felt good. When I was in Vietnam, my parents were very protective. They took care of me and helped me with everything from A to Z. But now, I don't fear anymore without them. I'm much more confident. (Thanh)

Thanh felt that studying overseas was an opportunity for her to get out of her comfort zone and grow up as an independent person.

When I lived with my family, I didn't think for others and was very sensitive. When people made me upset, I didn't want to meet them again. Here, I have to be really independent and deal with everything by myself, which helps me become strong. When I got out of the protection of my family, I realised that those conflicts helped me grow up. I think for other people. [...] I'm no longer a child. (Thanh)

Moving overseas brought her eye-opening experiences that would possibly change her life and perceptions of herself.

This decision has brought about a lot of changes to me, my life, and my view. Challenges make me stronger. I used to think that I was 'smart'. The previous environment made me feel like... I didn't go far, my perspectives weren't expanded, so I felt self-satisfied. But when I moved out of my community, I was nothing, not even a good student. I realised I need to learn more. (Thanh)

For some students, being away from family gave them opportunities to become a person with the ability to make their own decision. Ly, for example, was forced to decide on what to do when facing difficulties. However, her interview suggests that she considered this as an opportunity to lead her own life. She could do what she wanted, live where she was pleased, and eat her favourite food. She felt a need to be careful in every decision that she made because she had little experience and knowledge about life. Despite this, she acknowledged that overcoming difficulties helped her become a mature person.

[In Vietnam] I'm still dependent on my parents and not really decisive. Here, my situation requires me to make decisions about where to live or what to buy by myself. I need to think carefully about everything. That helps me grow up a lot. (Ly) 
Hai felt that living in a foreign country allowed him to lead a life that he longed for. In Vietnam, he was conscious of his role in a governmental agent and needed to follow the conventions or the norms of that role. However, in Aotearoa-New Zealand, he was a student and had more freedom to experience what he thought a governmental official would not do. A change of socio-cultural environment and status allowed him to escape from his job commitment and contributed to the freedom that he enjoyed.

In Vietnam, I wanted to experience new things. However, it was impossible because I was so busy with my work as a government agent. When I came here, I could do whatever I wanted and had new experiences. (Hai)

For other students, studying overseas was viewed as a journey to move out of their familiar communities and learn about new things. In this respect, Trung was representative of the participants. Coming from a rural area, he began his journey when leaving for a big city for his bachelor's degree, which he regarded as a journey of getting out of the village's bamboo range. That city, which he just knew about it through the newspaper or television, was unfamiliar to him. Reflecting on his experiences, he thought not living with family gave him the freedom to do what he wanted and develop fresh perspectives on the world. The further he travelled, the more he came to realise that he wanted to explore the world. This thinking contributed to his decision to study overseas. He wished to continue his journey outside "the village's bamboo range" to explore new things in a foreign country.

After high school, I wanted to get out of the village's bamboo range to attend a university in a big city. I thought I would get a stable job after getting my bachelor's degree but then, I wanted to have a postgraduate education and explore new things. [...] I imagined my life overseas, how it could benefit me, and I was excited. I was young. There is no reason why I should just stay in one place. (Trung)

\section{The ability to take care of one's self}

Besides the ability to handle problems and take care of themselves, the students emphasised the ability to manage their time and finance. Living in an unfamiliar country provided them with a chance to live independently but required them to be responsible for 
their own life. By finding ways to handle everyday issues, they became capable of thinking and acting for themselves.

The students were aware that they were living away from home and had no close friends alongside them. Although they made new friends, the networks could not replace the existing ones in their home country. The absence of familiar social systems significantly affected their living experience. Trung thought he needed to be more self-reliant and independent.

There are things here that I can't rely on my friends. [...] To overcome these problems, I have to try more and more. I think I have to confront them. (Trung)

Trung went on describing his situation, highlighting the need to be self-reliant through reiterating the crucial role of family:

In Vietnam, I have my family, relatives, and buddies with strong bonding, who can help me. When I'm sick, they can take care of me. But in New Zealand, if I'm sick, no one will help me. I have to get over it by myself. If you don't have good health, you have to learn how to take care of yourself. (Trung)

Ly appreciated the freedom she had when living by herself. She could control her life and decide what she thought was right for her.

In Vietnam, I don't have to do anything, even to cook. I just go to school or go to work. My parents always think that I'm still a child. So, I can't decide where to go but have to ask for their permission. I feel that I'm restricted because they control me. But here, they can't. Without my family, I'm free to make my own decision, about shopping, means of transport, where to live, and what to eat. I value that because I can learn many things. That's what I like. (Ly)

Self-care is not only about one's ability to meet their daily living needs (e.g., eating and sleeping) but also related to the identification of their unique needs and finding ways to take proper care of them. For most of the students, self-care was to take the time to do activities that could keep them healthy. Some (e.g., Bao and Thanh) were concerned about their 
health. While most did not consider their health issues as severe, they knew that anything could happen unexpectedly. They did not want their studies to be affected by these problems. The absence of their families contributed to their concerns. Unlike some local students, the students' families could not care for them if they became sick. Bao linked her concern about her health with her studies, explaining that women in their forties had different issues and could get sick more easily than younger people.

Apart from study issues, Bao's health issues were associated with her age. Being aware of these potential problems, she managed to take time for physical activities, which she considered a form of self-care:

At my age, I have different issues. I don't usually think about them but [they may affect me]. When I'm busy with my assignments and other things, I may feel tired or stressed. Normally, I take time to exercise every morning or evening to maintain my health. (Bao)

Entertainment, like watching movies and listening to music, benefited the students' mental health. Apart from gaining new experiences and exploring new cultural aspects, they boosted their mood and energy levels through these activities. Bao reported that she usually listened to music and went to the movie when she had time.

Other female students took care of themselves by participating in activities that were beneficial to both physical and mental health. Ngoc did not take part in these activities in Vietnam, but she did it here because she wanted to improve her health. She attended a yoga class at the Recreation Centre but then dropped out because she could not arrange her time. At the time of the interviews, she was attending a meditation class. She shared that she loved the class because the instructors were friendly, and most of all, it was good for her health:

I'm interested to learn about meditation. [...] I'm satisfied with the class. Instructors are changed every two weeks which brings me freshness. They're very nice and friendly. There're a lot of students, and sometimes no place for me, I have to stand at the back, but I still love it. My health improves a lot after one month. (Ngoc) 
The students felt a strong need to balance their work and studies. Studying overseas gave Ly opportunities to improve her time management skills and work-study balance. She felt grown-up when being able to balance work and studies.

I felt that I have been growing up a lot in my thinking. Here, I have to do everything by myself and allocate time for my studies and work. I can manage my time well, so one doesn't influence the other. I work seven days a week, but I still have time for reading articles and doing assignments. When I get my desired result, I feel like I have tried, and I value my achievement. I have become mature when I can manage to study well while working part-time. (Ly)

During the first six months, Thanh could not take care of herself. She had problems with her diet and health. She could not cook and did not know where to buy the food she wanted, which made her life difficult. However, she then learned to do things on her own:

Then I learned to do things by myself. I ate whatever I could cook. I never cooked in Vietnam. I boiled everything because it was quick. I thought I could live on omelettes, or boiled eggs but I couldn't eat them every day. [...] I learned to make various dishes and things started to get better. After 6 months, I got more confident. (Thanh)

Self-care also meant that the students knew where to seek assistance when facing difficulties. Thanh used the university counselling service because she did not feel well emotionally. She was confident that she became independent, could deal with difficulties herself, and knew where to get help:

I have become independent in many ways. I know how to deal with difficult situations, and I don't fear when having to do things on my own. [...] I need to rely on myself first. If I cannot help myself, I will seek help from others. (Thanh)

In the following extract, $V y$ described her journey of learning to live without her family. She shared that in Vietnam, she just needed to focus on her work because her parents took much care of her, cooked for her, and did all the housework. In Aotearoa-New Zealand, without her family, she felt a need to become a self-reliant person. 
I lived with my family, so I didn't experience much with things and life outside. Here I have to be independent. My friends can help me with minor problems but not bigger issues. They can give me advice but can't care for me like my family. [...] I have to go to school, work, and do all the housework at the same time, which made me independent. (Vy)

\section{Financial independence and ability to manage budgets}

Financial issues were a significant concern for the students. As indicated earlier, these issues could be seen in their choice of accommodation. In this theme, I focus on another aspect of financial issues - the sense of maturity and autonomy gained through becoming financially independent. In the beginning, the self-funded students relied on their parents for financial support because those parents wanted their children to be free from tension and not to be distracted from their studies by financial issues. However, they felt a need to earn their livings, which gave them a sense of independence. Thanh emphasised her determination to earn her own living.

I may have problems in the workplace like arguments with my boss or stressful work. I don't like the job but want to earn my own living. (Thanh)

In response to questions about their transformation, the students emphasised that their ability to manage their budgets contributed to their feeling of maturity. For most of the younger students, their parents were protective and supported them financially when they were in Vietnam. Being away from their families and living in a foreign country forced them to learn how to live within their means and decide on what they needed rather than what they wanted. Thanh, Tam and Vy shared:

In Vietnam, I didn't have to think about anything. But here, I have to consider how much I will spend on food and so on. I have a list. I will have to decide how much I will spend on this or that. I spend most of my money on food rather than less important things. I believe that if I eat nutritious food, I will have good health. Once I have good health, I can do everything. (Thanh) 
Before I came here, I stayed with my parents. But here, I can manage to support myself financially. (Tam)

In Vietnam, my parents supported me financially. I have become more financially independent here. I am trying to earn my own living and support myself. (Vy)

Another factor that contributed to the students' feeling of being an adult was learning to deal with their personal finances. For most of the students whose studies were either sponsored by their parents or scholarships, finance was not a problem. However, becoming independent from their parents' financial assistance or being able to contribute to their living expenses and tuition fees gave them a sense of growing up. Lan shared that she did not "worry about financial issues anymore" because she was employed. The following extract from interviews with Ly highlights her perception of the link between financial independence and personal grown-up:

I don't rely on my family or anyone else for financial support. I learned how to manage my expenses and to live on my budget. I can pay for everything myself and I feel that I am more grown-up. (Ly)

Coming from a humble background, Trung was under greater pressure than some other students to support himself. He reported that his paid work enabled him to cover his living expenses, meaning that his parents just needed to support his tuition.

I'm a self-funded student, so I'm under financial pressure. Besides studying, I have to work to contribute to my living cost which makes my life easier. I have a stable job and know how to manage my expenses. (Trung) 


\section{The Essence of Being Vietnamese Students in Aotearoa-New Zealand: Thriving Despite Adversity}

Lửa thử vàng, gian nan thử sức. Fire proves gold, adversity proves one's resilience.

- Vietnamese proverb

One purpose of an IPA study is to discover the essence of participants' experiences of a phenomenon (J. A. Smith \& Osborn, 2008). The students' stories were about a journey through difficulties and the process of self-development. They were nuanced and not always conscious. However, there was ample opportunity for the students to reflect on their experiences and bring a number of them into consciousness in the interviews. Looking back, they found that overseas education had changed them, in terms of not only knowledge and skills but also opportunities to become independent. They described their living and learning experiences as the two opposite sides of a coin: difficulties and chances for selfdevelopment. Multiple factors influenced their experiences such as language difficulties, the nature of their accommodation, and relationships with flatmates. These challenges forced them to become self-reliant. Reflecting on her experiences, Ngoc commented: "my experiences, both positive and negative, have enabled me to become mature". The following extract from Hai's interview highlighted the difficulties facing the students and their resilience:

Many people think that study abroad is rosy and exciting. But it isn't true. All international students know that it may be very tiring and stressful. If you don't have friends, you will have no one to share with. Usually, we don't want to share with our parents because we fear that it will make them worry. Most importantly, we want to gain good study results. (Hai)

The students felt more confident when they became familiar with the new living and learning environment. They were able to live without their families, support, and take care of themselves. Overseas education gave them opportunities to enrich their experiences. They believed in their ability to overcome difficulties. Their studies were their priority, and 
family was the driving factor for their ability to move in hard times. These findings showed that the students were not passive individuals but agentic beings, and they enacted agency in the face of challenges.

\section{Chapter Summary}

This chapter has presented and interpreted how the students made sense of their living experience concerning their homesickness and desire for family connection, contributing factors to their establishment of networks, and difficulties in living away from familiar networks. The findings suggested that all of them encountered difficulties when starting a new life. Some lived away from their families for the first time which contributed to their homesickness. Some expressed significant concerns about their families back home in Vietnam, which resulted in their feeling of not belonging. Most of them lacked motivation or found it difficult to form meaningful relationships with local students. Their primary network comprised co-national students. There was a sense that the students were illprepared for their independent lives in a foreign country. Some of them struggled to deal with difficulties without their families' assistance. However, reflecting on the difficulties, they commented that the challenges forced them to be independent and that their personal growth was worth those difficulties. In the next chapter, I will explain the findings and show how they relate to the literature on international students' experiences. 


\section{Chapter 9 | Discussion}

In this chapter, the findings are discussed in relation to the literature on international students' experiences. Smith and Osborn (2008) suggest that the value of an IPA study can be determined by the light it sheds in the wider context of the extant literature. In using IPA, I acknowledge that while each student's story was idiosyncratic or unique, there were commonalities in their experiences. In identifying the essence of their experience, the key findings highlight the students as agentic beings who could thrive in the face of adversity.

The discussion focuses on the key factors that shape the students' experiences and is structured in three sections: language issues, living experiences, and academic experiences. Linguistic issues were closely related to both their academic and everyday experiences and thus comes in a separate section. Regarding their living experiences, I focus on how they prepared for their life in Aotearoa-New Zealand, living arrangements, cultural identity in everyday settings, friendship networks, agency, cultural differences, and belongingness. In regard to their academic experiences, I will focus on heavy workload, difficulties in contributing to classroom discussions, and cultural identity in academic settings. Finally, I evaluate the contribution of the theories that formed the framework for the study.

\section{Language Issues}

The students' desire to improve their English motivated them to study in Aotearoa-New Zealand, an English-speaking country. However, language issues contributed to their difficulties, for example, communicating with others and understanding course content. Such issues limited cross-cultural interactions, especially with the host community. These findings are in line with previous studies showing that international students may encounter language difficulties both within and outside the university (Alsahafi \& Shin, 2017; Andrade, 2006; Khanal \& Gaulee, 2019; Park et al., 2017; Vu, 2013).

Language proficiency is a key contributing factor to the active human agency of many international students with a non-English speaking background (Sawir et al., 2012) and may 
affect their evolving sense of self and transition from the old sites to the new ones (Marginson et al., 2010). A lack of language fluency may contribute to their low level of selfconfidence and reluctance to reveal their communicative weaknesses by engaging in discussion, which potentially results in their being misinterpreted as being passive $(\mathrm{H}$. Li, 2017; Sawir et al., 2012). Students' feelings of insecurity, homesickness, and vulnerability are exacerbated by language difficulties (Nyland et al., 2009; Tian \& Lu, 2018). These challenges may make it difficult to engage socially with locals (Sawir et al., 2012).

International students' struggles with language have been documented in the literature. Kukatlapalli et al. (2020), for example, found that Indian students in New Zealand sometimes grapple with the local accent. Andrade and Hartshorn (2019) show that international students from an ESL (English as a Second Language) background may have extensive passive knowledge of the language but lack opportunities to use it to communicate in real life, contributing to their limited language ability. In reviewing the factors affecting international students' adjustment, Andrade (2006) suggests that teachers' accents, use of colloquial English, and rapid speech may be difficult for international students to understand. My study supports these findings. In the case of the Vietnamese students, language difficulties stemmed from their limited opportunities for interacting with English speakers in Vietnam. Most of them had a bachelor's degree in the English language and had learned English at least since secondary school. However, in Vietnam, few English teachers at universities were native speakers. Additionally, the English language taught in Vietnam differs from the one that they encountered in Aotearoa-New Zealand. In everyday life, there were a variety of accents and people may speak faster than teachers in Vietnam. This made it difficult for the students to gain familiarity with English as it is spoken in the country.

\section{Vietnamese Students' Living Experiences}

In this section, I focus on the students' preparation for life in a new country, issues with accommodation, cultural identity, friendship networks, student agency, cultural issues, and belongingness. 


\section{Preparation for life in Aotearoa-New Zealand}

The importance of pre-departure preparation was reinforced in this study. The students were poorly prepared for an independent life and initially had difficulties taking care of themselves, which impacted their experiences. The usefulness of advice, assistance or housing information from universities has been previously highlighted (Forbes-Mewett, 2018).

Students who undertake education in a country with different cultural practices from their own face significant challenges as they encounter unfamiliar norms in education and social life. If they are unaware of potential adjustment difficulties before their arrival in host countries and are unprepared for how different life will be, they are likely to struggle at the outset of their studies (Zhou et al., 2008). My findings support the need for a pre-departure curriculum designed to ease the cultural transition of students in their studies and everyday lives in a new country (Lamberton \& Ashton-Hay, 2015).

A study on the support needs of international students in England and the Netherlands found that students appreciated pre-arrival contact with peers, and information on accommodation (Bartram, 2008). In my study, some of the participants struggled to find accommodation and others got sick. The findings suggest that the students were provided with inadequate information on and support with accommodation, which impacted their housing and overall experience in Aotearoa-New Zealand. They thought the general information provided by the university was not useful in finding affordable accommodation. They saw the university as focused on students who wanted to live on campus. The accommodation search took place at a crucial stage of their transition into the new academic environment where they were overwhelmed with learning many new things. This left them little time to investigate the housing market. Also, they were bewildered by the differences between Vietnam's and Aotearoa-New Zealand's housing application processes.

\section{Issues with accommodation}

In contrast to previous research highlighting the academic, language and psychological adjustment experiences of international students, this study has deliberately explored the 
everyday living arrangements and experiences of a cohort of Vietnamese international students. Where one lives, which for these students was a temporary home, is ideally a place of refuge, security and where one can feel "at home". Home should be a place for them to enjoy a respite from the challenges of a new academic and social environment. The findings about the students' living arrangements and the impact on their studies clarify and support the claim that accommodation is a basic need that should be met before a person can address higher needs (Maslow, 1954; Schunk, 2012). If students have unpleasant housing experiences, their sense of security and belonging will be affected, and their studies are likely to suffer as a result.

The students encountered a range of difficulties in finding accommodation, especially when they first arrived in Aotearoa-New Zealand. These challenge those of Kukatlapalli's (2016) study about Indian students' experiences in New Zealand which suggest that, apart from having limited opportunities for interacting with local or other international students, the participants did not have difficulties relating to private accommodation. In addition to the intensification of the housing shortage in New Zealand, the differences between the two studies' findings may suggest that country of origin may be influential in different housing experiences. In my study, the students struggled to find an affordable and stable place to live. Reasonably priced houses were important because they reduced their financial burden. As a result, they ended up sharing their house or room with multiple people. In most cases, this was not what they wanted and was sometimes problematic. Tam, for example, shared her room with a mother and her child. Although she did not report any issues, she said that she would consider having a room for herself if she could afford it. Vy said that some of her flatmates were messy, and "things got worse" when there were more people in her house. The students' experiences were significantly affected by their living arrangements, especially with regard to overcrowded accommodation.

Apart from impacting the students' living experiences, issues with securing and affording a stable home might affect their studies. Financial hardship caused most of them to opt for affordable but unsatisfactory housing rather than expensive accommodation. This finding supports those of Marginson et al. (2010) and Forbes-Mewett (2018) who suggest that international students' choice of accommodation is associated with their financial 
situations, and that low-cost housing may lead to adverse experiences. In this study, unpleasant housing experiences contributed to their decision to move out. Finding and moving to a new place required time and effort. As indicated in Chapter Six, some of the students (e.g., Vy, Hai, and Bao) reported that their flats were noisy and did not provide an appropriate workspace. Usually, they had to study in the university library. Some of them changed accommodation two (e.g., Ngoc, Vy, and Ly) or up to three times during their oneand-a-half-year stay in the country (e.g., Thanh and Hai).

Other housing-related issues, including invasion of privacy, low levels of personal security, lack of personal space (e.g., students who stayed in living rooms or who shared rooms) and renting without a written tenancy agreement, potentially made the students vulnerable. The two female students (Ngoc and Thanh), who were concerned with their privacy and personal security, ended up moving out. It was difficult for them to secure affordable and safe housing because they lacked knowledge about renting regulations and had little choices in an expensive rental market. These results support findings from earlier studies (Ike, Baldwin, \& Lathouras, 2017; Obeng-Odoom, 2012). International students' experiences were different from those faced by many domestic students because of various contributing factors (e.g., unfamiliarity with the local housing market and renting laws), and unpleasant experiences can have more severe impacts on their lives and studies. When they have problems with rental housing, they cannot move back to live with their families like a number of local students.

As indicated in the introductory chapter, I completed most of the study before the coronavirus was first reported and the disease spread globally. Amid the COVID-19 pandemic, international students have been identified as among the most vulnerable groups in tertiary education due to challenges such as lack of accommodation facilities, financial issues, mental health problems and travel restrictions (Sahu, 2020; Ziguras \& Tran, 2020). In many countries that host a large number of international students (including Vietnamese students), such as the USA, the UK, Australia, Japan and South Korea, the situations are still characterised by great uncertainty. As those countries head further into the crisis, the importance of accommodation in students' wellbeing is more pressing. Crowded housing with shared facilities creates an environment that can spread disease 
within a house. This is particularly concerning when students are living in cramped conditions because it is impossible to self-isolate. Coupled with geographical distance from their familiar networks, national lockdowns and travel restrictions in many countries aimed at containing COVID-19 will probably hinder international students from achieving assistance that normally they would expect to receive if they were in their home countries. These problems suggest an urgent need to address the housing issues of international students. Focusing on the Vietnamese students' housing experiences, my study has made valuable contributions to these critical issues.

\section{Cultural identity in everyday settings}

The students strived to maintain harmonious relationships with people from different cultural backgrounds. They tried to be tolerant and avoid conflict and tensions. Some controlled themselves and accepted their flatmates' cultures and lifestyles. For example, when Hai's flatmates, who were not Vietnamese, held noisy parties, he would go to the university library. Both Lan and Hai strove to keep their emotions in check to maintain good relationships with their flatmates. Acceptance of individual differences is an aspect of collectivistic nature of Vietnamese culture. In collectivist societies, relationships with other members of a group or a community play a central role and people tend to prefer resolutions that will not harm relationships (Hofstede, 2011; L. T. Tran \& Marginson, 2014). Vietnamese culture with a blend of Buddhist, Confucian, and local values emphasises the role of harmony and tolerance in relationships amongst people (Q. T. N. Nguyen, 2016; L. T. Tran \& Marginson, 2014). A well-known Vietnamese proverb says: "Một câu nhịn là chín câu lành" (One compromise results in nine blessings), highlighting the benefits of tolerance in life. Valuing harmonious relationships and avoiding confrontation was prominent in the students' accounts of relationships in flats.

Apart from these cultural values, international education and cross-cultural experience might play a part in shaping the students' behaviours. Overseas study potentially provides students with opportunities to reconstruct their identities through immersion in a new cultural environment (Marginson, 2014; B. T. T. Nguyen \& Pennycook, 2018; L. Nguyen, 2018). Respect for cultural differences is part of cultural inclusiveness within international 
higher education settings (McLoughlin, 2001) and, in this study, comes from the students' experiences in Aotearoa-New Zealand. Marginson et al. (2010) assert that harmony is associated with 'inclusion, membership and acceptance', and is essential to international student security (p. 392). According to Berry (2017), intercultural adaptation refers to individuals' ability to establish relationships with others from different cultural backgrounds. Tian and Lu (2018) suggest that overseas study has a positive impact on international students' autonomy, which is manifested in the improvement of their abilities to initiate, monitor, and control their behaviours. These ideas imply that the Vietnamese students' behaviours may be affected by their inter-cultural experiences. Their identities were constructed based on their home country cultures and their overseas educational and crosscultural experiences.

The findings highlight the importance of cultural identity in shaping the students' experiences. This can be seen in their feelings as Tết (Vietnamese Lunar New Year) approached, and in their longing for family connection. Tet is one of the most important traditional celebrations in Vietnam. One student was delighted to celebrate Lunar New Year and have Vietnamese traditional dishes. A Vietnamese folk song says: "Dù ai buôn bán nơi đâu. Nhớ đến ngày Tết rủ nhau mà vè̀" (Wherever in the world you are conducting business. Remember to remind each other to return home for Tet celebration) denoting the significance of this festival deep-rooted in the minds of the Vietnamese. Those cultural factors have shaped the students' cultural identity and influenced their attitudes and interactional strategies in daily life.

Many students had strong connections to their families, which has been identified as a characteristic of Vietnamese culture. Vietnam is a collectivistic society that is characterised by strong family ties (Hofstede, 2011) with family members being interdependent and tightly knit (Cultural Atlas, 2020). People typically feel a strong sense of belonging to their families and value cohesiveness of the family unit. The need to connect with family is deeprooted in Vietnamese culture and shaped students' behaviours and responses to challenges.

Work was an integral part of the students' lives and identities. Their jobs contributed to their sense of who they were. Major changes in work could therefore have an emotional impact 
on them. Vietnamese people generally accept hierarchies associated with education and work (Cultural Atlas, 2020). Accordingly, highly educated people and people who work in some sectors (e.g., education and the civil service) are respected. Loss of status could affect students' connectedness and identity (Dang \& Tran, 2017). In this study, while most of the participants were academics in Vietnam, they worked in restaurants in Aotearoa-New Zealand. There was a sense that the loss of social status associated with these occupations triggered grief for some of them (e.g., Thanh). Such feelings played a part in their decisions to return home after graduation. However, others enjoyed the change from their professional work in Vietnam because it gave them a chance to experience new things. For example, Hai shared that leaving his role as a governmental official in Vietnam allowed him to have more freedom and participate in activities that normally he would not do in Vietnam. Despite the differences in the impact of occupational changes on their feelings, the students regarded this as an opportunity to get a fresh perspective from being an international student and taking different occupations, which contributed to their identity development. The students changed as they adopted new perspectives or values. This is consistent with Berry's ideas about acculturation experiences.

\section{Friendship networks}

Ability to form new relationships, whether with co-nationals, locals or international peers, may affect international students' security (Marginson et al., 2010). The students' friendship networks mainly comprised Vietnamese international students and/or expatriates, except for the male student from Political Science who established good relationships with flatmates from other countries. He was an outlier because he had lived and worked in different regions in Vietnam and had opportunities to interact with people from different socio-cultural backgrounds. Another reason was that he desired to make friends with people from other cultures. These findings are congruent with many studies on international students' networks. Language proficiency may affect EFL international students' ability to establish networks and that their network comprises same-culture or similar-culture people (Marginson et al., 2010; T. T. T. Nguyen, 2019). International students sometimes find it difficult to separate themselves from co-national networks and can easily engage only with their co-national friends if they do not actively seek opportunities to socialise with local 
students or other international students (H. Li \& Pitkänen, 2018). In this study, the students' relationships in Vietnam were affected when they studied overseas, and they felt a need to establish new networks in the host country. Eight students from the twinning programme came to Aotearoa-New Zealand together and challenging situations brought them closer. These friendships ran less deeply than their relationships with family members but were more readily accessible when needed. Time played a role in shaping the students' networks. They stayed in the country for a relatively short time, which made them reluctant to form meaningful relationships with non-co-nationals. Therefore, their networks mainly included co-nationals, those with whom they interacted on a daily basis.

There are advantages and disadvantages to this tendency to remain inside co-national networks. Co-national friends may provide practical and emotional support. This is consistent with findings from earlier studies (Marginson et al., 2010; L. T. Tran \& Gomes, 2017) which emphasised the support provided by students of the same nationality. In this study, international students' co-national friends helped them to deal with loneliness and provided important support and resources for their resettlement in Aotearoa-New Zealand. Further, there was a sense that beyond support, in some cases, their friends were more than a network. Thanh's friends, for example, took care of her when she was in the hospital. They formed a temporary family and jointly made their flat become a "safe" place where they could take refuge from the stress of their classroom and daily lives.

Despite these benefits, the students reported that the co-national network sometimes hindered them from forming relationships with other international or local students. These findings are supported by previous research. With a lack of meaningful interactions between different student groups, international students may have few opportunities to improve their English and enrich their educational, social, and cultural experiences (H. Li \& Pitkänen, 2018; Marginson et al., 2010; Sawir et al., 2008). In this study, within co-national networks, the students spoke English less frequently and this also had the effect of limiting their contact with local culture, and this perhaps increased their sense of distance and not belonging in Aotearoa-New Zealand. In this regard, having part-time jobs provided the students with opportunities to use English through interacting with co-workers and customers, which increased their confidence and proficiency in English. 


\section{The students as agentic beings}

Living in a foreign country opened up opportunities for the students to reconstruct their identities. This is consistent with a growing body of literature that addresses the selfformation of international students (Marginson, 2014; L. T. Tran, 2016). Students can gain a better understanding of different cultures, which in turn enhances their self-confidence, maturity and adaptability (Maharaja, 2018; M. N. Nguyen \& Robertson, 2020). Ye and Edwards (2015) explored the experiences of Chinese doctoral students in the UK and found that they used various coping strategies to overcome challenges and that their lives in the foreign country were characterised by self-reflexivity, creativity and autonomy. In my study, the students actively engaged in constructing their self-identities by learning to deal with difficulties, improving themselves, and determining to achieve their desired goals. Trung regarded his overseas study as a journey enabling him to "get out of the village's bamboo range" and broaden his horizons. Younger students thought that their changes potentially helped them become better versions of themselves.

The students had the freedom to do what they wanted and to become who they wished to. Living away from their familiar environments and people allowed them to have new experiences. This is consistent with Marginson's (2014) construction of agency freedom. Striving to deal with difficulties in daily life proves to be a process of becoming independent individuals. This is consistent with previous literature on international students' experience. Dentakos et al. (2017) suggest that problems facing students may be related to developmental transition, such as becoming independent, taking care of themselves, and a feeling of being unprepared for confronting challenges. The findings reveal that the Vietnamese students experienced transformation and became independent after living and studying abroad. Thanh, for example, maintained that being away from her family enabled her to become mature and that she was "no longer a child" and Ly needed to make decisions by herself and take responsibility for her life. Through this challenging process, they became more confident in their ability to live independently.

As discussed in Chapter Two, Marginson (2014) maintains that international education is considered as "a process of self-formation within conditions of disequilibrium in which 
student subjects manage their lives reflexively, fashioning their own changing identities, albeit under social circumstances largely beyond their control" (p. 6). Agency is a key contributing factor to the acquisition of skills and the formation of new perspectives and identities, and these skills and knowledge were formed during their studies and sustained over time (Gu \& Schweisfurth, 2015; B. T. T. Nguyen \& Pennycook, 2018). The findings from this study support Marginson's statement and highlight the critical role of the students' agency in enabling them to become independent individuals.

Most of the students, especially the younger ones, enjoyed their independent lives and valued their freedom in Aotearoa-New Zealand. However, they also felt homesick and wished to maintain a connection with their families back home. Some of them missed the familiarity and security of being part of a collective and interdependent unit (i.e., their extended families). It is noteworthy that, for most of them, this was the first time they lived in a foreign country, thousands of miles away from their families. Their homesickness was understandable because it has been found that students who leave their homes for the first time may encounter more difficulties than those who have lived away from home before (Sawir et al., 2008). Besides, there was a sense that the students valued their cultural identities and maintained them through strong family relationships. Such connections have been shown to allow the students to strengthen 'continuity of self' and sustain a sense of self (Marginson et al., 2010, p. 360).

\section{Cultural differences and cultural similarities}

Many of the Vietnamese students encountered difficulties in knowing how to respond in certain situations and understanding cultural differences. In social events, international students may face unfamiliar communication patterns which can lead to 'awkwardness' in communication (C. Smith, 2020). In this study, Hai struggled to understand stories or jokes related to local cultures. The students commented that there were marked cultural differences between Vietnam and New Zealand. This perceived cultural distance, which is usually noted in intercultural contact (Berry, 2017), impacted on the students' ability to communicate successfully in the new living environment. In the context of international education, the ability to operate effectively in an unfamiliar environment is a critical factor 
in human security (Marginson et al., 2010). Cultural issues potentially affect international students' self-confidence and their capability for human agency. Kukatlapalli et al. (2020) found that some Indian participants had difficulties understanding New Zealand humour. While expressing a desire to broaden their networks, my participants were discouraged by challenges associated with cultural differences. They needed a sense of confidence when communicating or interacting with others, but described incidents that affected their confidence, which in turn, had a negative impact on their ability to build new networks.

Cultural differences were usually reported as barriers to forming meaningful relationships with local students. This finding is consistent with earlier research (Belford, 2017). In the current study, there was a lack of understanding of each other's cultures. The students' limited cultural knowledge affected their relationships with host nationals. This is reflected in their struggles with cultural differences and their reluctance to develop relationships with local students. Tuan, for example, sensed a gap between him and his classmates who were local students and he could "hardly find a common ground" with them. This contributed to their sense of not connecting with members of the local community.

The students' close friendships were usually with co-national peers with whom they shared a similar cultural background. They received emotional and practical support from their friends. This support enabled them to deal with negative feelings (e.g., homesickness and loneliness). They felt that people from other cultures might have different views about cross-cultural experiences and might not understand what they experienced. For example, Trung reported that he preferred to have Vietnamese friends because they shared "the language, culture, and similar viewpoints" and they understood his experience. When interacting with others, the students wanted to feel comfortable and confident, which could be achieved through having a shared cultural background.

\section{Belongingness}

Sense of belonging is an important need for international students. In Maslow's hierarchy of needs, the need for belonging should be satisfied before individuals reach the levels of esteem and self-actualisation (Maslow, 1987). Feeling of belonging is important because it may affect their overall living and learning experiences (Maslow, 1987; Schunk, 2012). 
Belongingness is related to feelings of acceptance, connectedness, being needed, and fit within the environment they are immersed in (Hagerty \& Patusky, 1995). In this study, the students' 'belongingness' was associated with a range of issues (e.g., accommodation issues, homesickness, concerns about families, and limited networks). Housing insecurity, which potentially led to the students' lack of feeling 'being at home', was related to difficulties in finding and securing affordable quality accommodation, issues in negotiating relationships with flatmates, noisy and crowded housing. They felt that they had 'no place' in New Zealand. However, this feeling was reduced by their co-national friends who, in helping each other, created 'a temporary family'. Homesickness and concerns about families at home were a source of anxiety, which potentially affected their health and studies. Bao described her homesickness as 'bacteria'. Such issues collectively contributed to their feelings of not belonging and led to their decision to return home after graduation. Knowing that the final course was delivered online, Tuan decided to go back to Vietnam before completing his studies. Having few friends who were local people suggested that they had weak connections and were not part of the local community. Such issues potentially made them vulnerable and affected the quality of their lives.

\section{Vietnamese Students' Academic Experiences}

Learning in a new and unfamiliar system was challenging for most of the students. In this section, I focus on the difficulties facing them in the academic setting, including heavy academic workload and classroom discussion. I also discuss how the students brought their cultural identities and values into the academic settings.

\section{Heavy workloads}

The findings showed that it was challenging for the students to deal with heavy workloads that were related to their preparation before class and big assignments. Not completing set readings contributed to their difficulties in understanding lectures. Bao associated her health issues with her heavy workload. These findings match those observed in earlier studies, where the participants struggled with academic workloads, work-life balance, assignments, and that producing written work in a foreign language took up much time because this involved several drafts before completion (Heng, 2018; Lem, 2019; Naylor et 
al., 2018). A number of my participants found it challenging to complete a large amount of reading before class. They compensated for their limited understanding of teaching content by spending more time on self-directed study and seeking support from their Vietnamese classmates. Coupled with issues in daily life (finding accommodations, issues with living arrangements), heavy workload contributed to their feeling overwhelmed.

The students wished to have good academic outcomes and prioritised their studies. Their workloads left them little time to engage in social activities. This finding confirms Li's (2017) study showing that when students find their subjects demanding, they may spend less time on social activities. In this study, the students wanted to complete their programmes within the time frames. Their academic priority, heavy workloads, and lack of motivation in social engagements hindered them from connecting with others and expanding their networks.

\section{Issues with classroom discussions}

Apart from heavy academic workloads, a number of the students encountered difficulties when contributing to class discussions. This study supports Li (2017) and Smith (2020), who identified language skills as a key contributing factor for students' silence in the class. Speaking and listening are essential components of classroom interactions. In this study, limited listening skills made it difficult for the students to follow class discussions while language difficulties prevented them from fully participating in such discussions. They had to search for appropriate words and consider how to express their ideas. Furthermore, speaking before strangers was challenging and a source of anxiety for some. Concerns about making grammatical or pronunciation mistakes and classmates' failure to understand what they said made them reluctant to contribute to discussions.

The participants may have been affected by their prior learning experiences and home culture. Unfamiliarity with group discussions contributed to their limited engagement in classroom activities. In Vietnam, teachers do not always seek to design classroom activities that engage students in discussions (Das et al., 2019) and cultural values do not encourage debate or discussion with teachers (Mai \& Hall, 2017; Nghia et al., 2020). It appeared that some of the students in this study were not familiar with such teaching practices and were 
ill-prepared to study in environments where discussion with classmates and teachers was the norm and speaking in front of class encouraged.

\section{Cultural identity in academic settings}

To understand the students' experiences, it is important to explore how they maintained their identities in academic settings. This is associated with how the students chose to acculturate in the new environment (Berry, 2017). The students' cultural identities were seen in their behaviours in academic contexts. In Vietnam, the relationships between students and teachers are usually formal and students should not challenge their teachers (Harman et al., 2010; Mai \& Hall, 2017). In this study, the students valued the close relationships with their lecturers, something that might be different from what they were familiar with in Vietnam. Despite this, the students tended not to confront their lecturers about issues. For example, although Lan was unhappy about her lecturer's teaching style and felt frustrated, she did not talk about this with that lecturer. Most of the students usually chose to discuss academic questions with their classmates rather than to seek guidance from their lecturers, which made their friends become their main source of support in the classroom. This could be because they were more familiar with formal relationships with their teachers in Vietnam and might feel uncomfortable talking with them. Another reason might be that it was more convenient for them to meet and talk to their friends. Also, most of the students established closer relationships with Asian students than local ones because they found common ground and had "similar thoughts and cultures". Such practices and cultural values ingrained and affected their attitudes in the new academic environment.

\section{Major Contributors to the Vietnamese Students' Experiences in Aotearoa-New Zealand}

The findings show multiple factors impacting the Vietnamese students' experiences (see Figure 9.1) and highlight the critical role of housing security in the students' experiences. While accommodation is usually conceptualised in the literature on international students' experiences as part of their everyday experiences, in this study, it stands out as an influential contributor to their experiences, including their studies. Although the students travelled to New Zealand for study purposes, accommodation became one of their first major concerns 
and priority after they arrived in the country, as identified in Chapter Six. Finding a suitable place to live was among the first fundamental steps when starting a new life. Most of them reported a lack of assistance and information from the university about private accommodation, which they especially needed when they first came to New Zealand and were unfamiliar with the new living environment. They struggled to find a place to live given their financial constraints and felt vulnerable due to limited knowledge of the local housing market and tenants' rights. Housing issues (e.g., finding suitable accommodation, limited living spaces, and concerns about privacy and personal security) were common and remained throughout the time of some students in the country. Under some circumstances, having positive housing experiences became as important as learning experiences.

In everyday settings, some of the students lacked preparation for living in New Zealand. Cultural values and identity shaped their responses to and attitudes towards challenges. Other issues were related to language barriers, cultural dissonance, and difficulties in forming meaningful relationships. Homesickness, housing issues, and worries about families negatively affected their sense of belonging. In academic settings, they struggled with new and unfamiliar teaching and learning styles, heavy academic workload, and referencing.

The students' experiences were complex because each element of their living and academic experiences interrelated with the others. For example, Vietnamese cultural values and identity were reflected in how they negotiated relationships with their flatmates and affected the way they engaged with host country communities and academic practices. Language issues were a challenge both within and outside of the university, and affected many aspects of their experiences, such as interactions and connection with members of the host community and understanding course content.

After getting through such tough times, the students became independent individuals. Such independent selves were gained through enacting their agency, which was one of the aspects of experience that they most valued. While maintaining their home country identity, the students became accustomed to new practices and incorporated new elements (e.g. academic norms and conventions) from their New Zealand academic experiences into their academic identities, melding Vietnamese and New Zealand identities. 


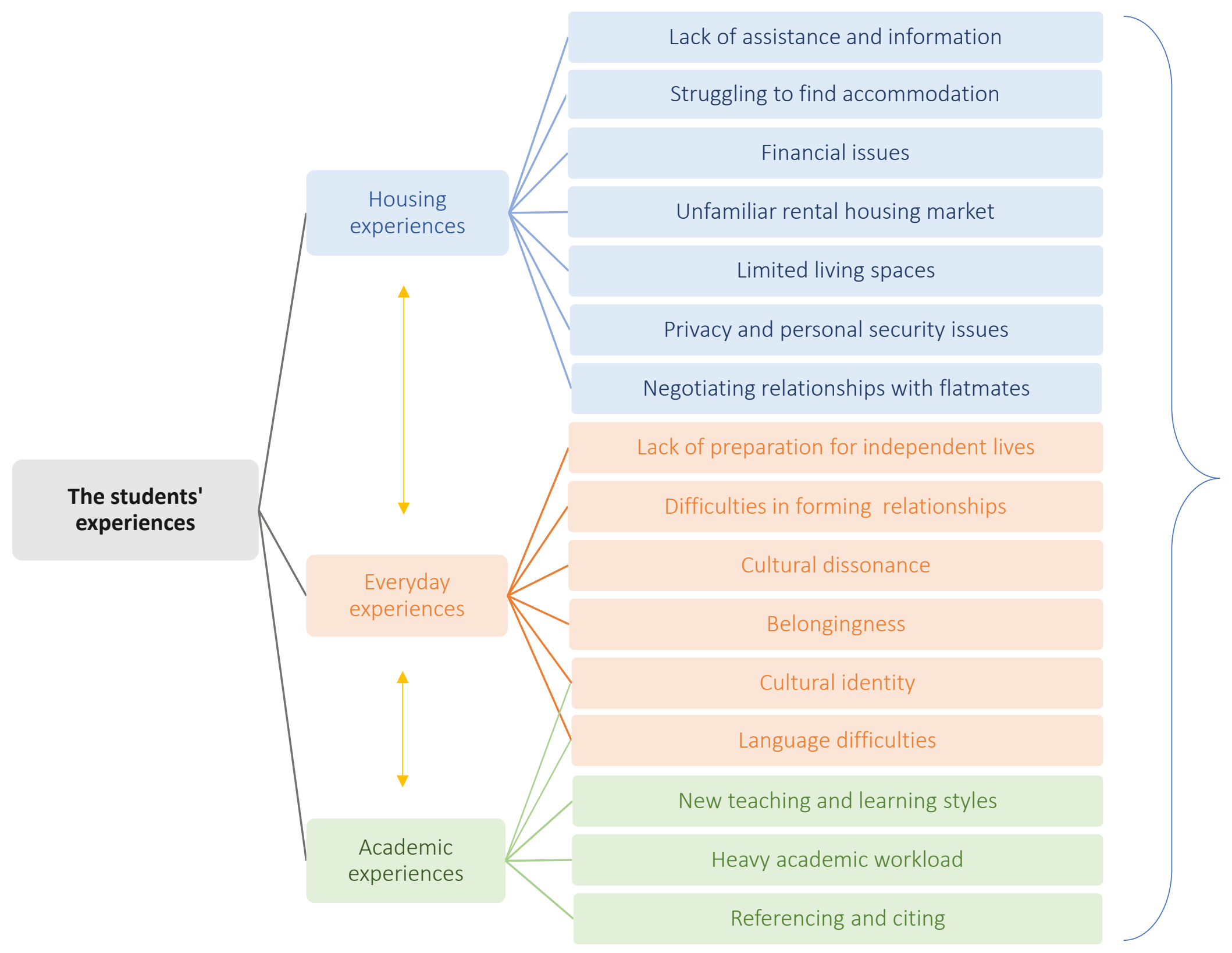

Independent selves and the melding of a New Zealand and Vietnamese identity

Figure 9.1. Major contributors to the students' experiences 
Within the context of New Zealand's international education where the government has implemented the Code of Practice for Pastoral Care of International Students, or The Code (New Zealand Qualifications Authority, 2016) and International Student Wellbeing Strategy (New Zealand Ministry of Education, 2017), the findings about the students' accommodation are relevant and important. The Code emphasises that signatories are responsible for international students' health and wellbeing associated with accommodation, and that relevant advice and information about accommodation (e.g., tenants' rights and obligations) are accessible to them. In considering the expected outcomes, the International Student Wellbeing Strategy highlights students' knowledge of rights about accommodation as part of economic wellbeing. Students' security, welfare and needs are taken into account at the institutional level. With this in mind, I provide recommendations for education providers in the next chapter.

\section{Evaluating the Theoretical Framework}

In this section, I consider how the theoretical framework based on the four theories - Berry's (2017) acculturation framework, Marginson et al.'s (2010; 2011) conceptualisation of human security and agency, and Maslow's (1970) idea of human needs - worked with this study. Although L. N. Tran's (2011) acculturation framework was not utilised in this study, it is useful to discuss its applicability to my study due to its close relation to Berry's acculturation framework and connection with Vietnamese students.

The framework was useful and comprehensive because it enabled me to interpret the experience of the Vietnamese students and look at it through various lenses. With the acculturation framework, I could see what the acculturation process was like for the students and what factors had an impact on their acculturation experiences. The notion of human security from Marginson's idea of international student security and self-formation gave me a platform to look at how the participants were both affected by environmental factors and were self-determining agents. Through the lens of Maslow's hierarchy of needs, I realised that as human beings, the students had needs to be satisfied (e.g., needs for belonging and shelter). One of the ultimate purposes of overseas study was to gain 
academic credentials. However, without acknowledging the students' basic needs and security, it would become difficult for them to achieve such goals.

In relation to Marginson's idea about identity, the students' identity is shown through their responses to issues in everyday settings. They embraced their home culture identities and had a strong connection with their families back home. In treasuring such identities, they were motivated to overcome challenges. Living and studying in another country enabled them to acquire new knowledge and have experiences that they did not have in Vietnam. In this way, they could (re)construct their identities. Further, there was a sense that some students (Hai and Trung) accepted and acquired new identities through their exposure to unfamiliar environments. Within the context of their living arrangements, this relates to their respect for cultural differences in their flatmates' lifestyles and to their efforts to accept such differences.

In different contexts, the students might use different acculturation strategies. In everyday settings, a number of them exclusively lived or mainly interacted with co-nationals, who assisted them in tough times and gave them a feeling of 'home away from home'. This choice could be categorised as 'separation', but that seems a misinterpretation of the students' intent. There was a sense that they thought integration, which refers to individuals' maintenance of heritage culture and at the same time, efforts to establish relationships with host communities, was useful to them. Tuan, for example, emphasised a need to "learn from each other" and "understand" people from different cultural backgrounds. This bi-directional process ideally involves each cultural group incorporating aspects of the other's culture and practices but there was little evidence of such initiatives from the local 'cultural group'. Nor did any of the students express adopting marginalisation strategies. A more apt interpretation of their choices is that of fitting in. A reason for this is that to achieve their purposes of overseas education, one of which was to acquire language skills and understanding of local cultures, they felt a need to engage with host communities.

In academic settings, elements of the students' practices appeared to be consistent with integration and assimilation strategies. They wanted to fit into the new academic environment. They made friends with both Vietnamese and other Asian international 
students. While they wanted to establish connections with the local students, they had few local students as friends. They acknowledged that local students contributed to forming their academic identity through observing the way the local students participated in discussions, raised questions, and hearing their perspectives. Their Vietnamese cultural identities were seen in their attitudes towards issues, for example, their avoidance of confronting or challenging their lecturers. However, they were interested in and open to the new academic conventions and norms such as academic citations and the closeness of the relationships between lecturers and students. Their openness was understandable because in deciding to study overseas, their major purposes were to gain a qualification and to acquire skills and knowledge. Overseas degrees and knowledge are valued in Vietnam and potentially improve career prospects and opportunities. There was a sense that the students were not satisfied with Vietnamese higher education, which was a driving factor for their academic journeys. These factors collectively contributed to how they engaged with their lecturers and peers in the academic environment.

Despite its comprehensiveness, the applicability of the acculturation strategies from Berry's framework to my study posed challenges. It was designed to explore the experience of immigrants and refugees. I could see potential limitations of this model for shorter-term sojourners such as international students. At times, I struggled to fit my participants' experiences into the acculturation patterns. This is likely because international students may be less involved with local communities than immigrants because of their short time in host countries (Berry, 2017). In this study, I interpreted the acculturation patterns with caution. The students from the twinning programmes stayed in New Zealand for one and a half years. After finishing their studies, two out of the ten participants remained in the country on three-year work visas while the rest returned to Vietnam. The students were conscious of their relatively limited time, and their priority was to complete their studies within the timeframe. Most anticipated that with geographical distance, it would be difficult to maintain friendships with non-co-nationals. In this context, it is hard to conclude that they preferred a particular acculturation strategy. Their adoption of any strategies did not necessarily reflect what they wanted to do. If they had had more time in New Zealand, they would be likely to change their lifestyles and behaviours. Reflecting on their experiences, some of them said that they would like to focus more on their studies to obtain better 
academic results. Others shared that they wished to enrich their experiences by travelling to as many places as possible and to make friends with more local people. They were constrained by circumstances and did not have the luxury of time to pursue new interests and friendships. These findings suggest that what seemed to be their preferred acculturation strategies at the time of this study might not be suitable strategies for them in the long run, and such strategies were subject to change over time.

The heritage and host cultures had a significant impact on the students' experiences. Their behaviours were shaped by home cultural values. This is seen in Hai's delight when he celebrated Tet holiday with the landlord's family. He sought to establish a connection with Vietnamese values through this celebration. The students perceived significant differences between home and host cultures. Such differences affected their perceptions of themselves and interactions with people from different cultural backgrounds.

With respect to L. N. Tran's (2011) acculturation framework for Vietnamese international students, I found that a number of its new elements, including the history of Vietnam, perceived attitudes of Vietnamese immigrant communities towards Vietnamese international students, and cultural mistrust had limited applicability to the situations of the students in this study. This was in part because New Zealand has a much smaller Vietnamese community than Australia has. While some of the students reported that they knew some local Vietnamese in New Zealand, they had few interactions with them and did not feel discriminated against by those people. None of them commented on political issues or on attitudes towards communism, and thus I have no evidence to show that such issues related to Vietnamese socio-political context and history were relevant to them.

In L. N. Tran's framework, cyber integration and acculturation dilemma was highlighted. In my study, the students did not report on instances of cyber integration. They were perhaps too busy with their studies to seek vicarious online acculturation experiences. I was cautious about utilising the concept of acculturation dilemma in my study. In Tran's study the dilemma in terms of interaction related to the distrust of the local Australian groups (Vietnamese and other Australians). In contrast, my findings revealed that for some of the students, there was a mismatch between their acculturation attitudes and behaviours. For 
example, although they wished to engage with the local community and to have local friends, they lacked the time to act on this. Prior to analysing my data, the acculturation dilemma had appeared to be useful for interpreting experiences. However, as explained earlier, due to a number of constraints (e.g., time constraints), the students' behaviours (or acculturation strategies) were not stable and were likely to change over time, which as a result, would not lead to an acculturation dilemma.

Maslow's and Marginson's ideas about human needs and security fit well with my study. In line with these ideas, the findings show that students' needs in terms of accommodation, support networks, and personal security had to be satisfied. Renting without a written agreement made some of them vulnerable. Anxiety about personal security was apparent and related to the female students' experiences with their flatmates. Having co-national friends made the students feel safe in a new and unfamiliar environment. They felt relief to have someone to share their unpleasant experiences with and to seek advice from. If such needs were not satisfied, their overall experience was likely to be affected.

While the students encountered a range of challenges, they actively strived to find ways to deal with them. Looking through the lens of Marginson's human agency, this is associated with students' agency. For example, with respect to their living arrangements, the students attempted to maintain harmonious relationships with their flatmates. They were conscious of the possible impacts of their behaviours on these relationships and actively sought to control their emotions. Their agency was consciously relational and required the subjugation of their personal preferences for the harmony of the group, and thus reflected Vietnamese values and identity.

In brief, there are differences in the levels of the applicability of the theories to this study. Due to considerable differences between immigrants - the target subjects of Berry's acculturation framework - and international students, the suitability of this framework to explore Vietnamese international students' experiences is limited. The acculturation framework, especially the four acculturation strategies, may be more suitable for studies on the experience of students who stay in host countries for a longer time. Marginson's 
conceptualisation of human agency and security and Maslow's hierarchy of needs fit well with my study.

\section{Chapter Summary}

I have highlighted the key findings in relation to the literature about international students and outlined some challenges that Vietnamese students face when pursuing university education in another country. Students' attitudes and responses to challenges are shaped by a range of cultural factors. Accommodation and living arrangements had a significant impact on this group of students' experiences. Language difficulties exacerbated the issues facing them in both academic and everyday settings. The differences between Vietnamese and Aotearoa-New Zealand education systems (heavy academic workloads and preference for classroom discussions) caused them difficulties. While struggling to handle problems, they had opportunities for transformation. In the next chapter, I discuss my study's contributions, outline its strengths and weaknesses, directions for further research and implications for universities and students, present my reconceptualization of the framework, and provide a reflection of my experience as a researcher. 


\section{Chapter 10 | Implications and Conclusion}

One's destination is never a place, but a new way of seeing things.

- Henry Miller

In this chapter, I summarise the key findings, discuss the theoretical contributions of this study, and describe how I reconceptualised the framework. I argue that housing security was associated with the students' satisfaction with their accommodation and was central to their wellbeing and sense of belonging. The strengths and weaknesses of the study are also addressed. I outline some of the implications for both universities and international students. Finally, I conclude the chapter with a reflection on my research journey.

\section{Summary of Key Findings}

The purpose of the study was to explore how Vietnamese postgraduate students adjusted to academic and social lives in Aotearoa-New Zealand. I set out to investigate the following question: 'What are Vietnamese students' lived experiences of everyday life and study in Aotearoa-New Zealand whilst they complete a master's degree?' In posing this question, I was interested in understanding how Vietnamese students made sense of their living and studying experiences and wish to capture the essence of their experiences.

Although Aotearoa-New Zealand universities host an increasing number of Vietnamese students, little is known about their experiences concerning living circumstances and few studies focus specifically on Vietnamese international students' experience. No research has focused on Vietnamese master's students in New Zealand. Although studies on Vietnamese students in other countries provide insights into their experiences, there are fundamental differences between New Zealand and those countries. Addressing this gap, my study has offered insights into the living experience of Vietnamese students in Aotearoa-New Zealand.

I identified seven superordinate themes that captured the essential meanings of 'what it is like' to be a Vietnamese student in Aotearoa-New Zealand. The students encountered a 
range of difficulties. In everyday settings, they wanted to connect with their families and were worried for them (e.g., children and elderly parents). It was challenging to build meaningful relationships with locals. They confronted issues in relation to their living arrangements. It was demanding to find a place to live and to have a roof over one's head because they had limited understanding of the renting laws and little knowledge of the rental market which potentially put them at risk and made them vulnerable. Their living spaces were often overcrowded and noisy, which posed problems. The students struggled negotiating relationships with their flatmates. A number of the female students reported on issues related to privacy and personal security. Such problems negatively affected their housing security and contributed to their anxieties, unrest, dissatisfaction, and lack of comfort in their houses. There was a sense that the students were ill-prepared for their new lives. However, they became accustomed to living in the new environment and more independent as time passed.

In academic settings, the students encountered a range of issues, including following lectures, understanding academic texts, and doing assignments. Differences between the Vietnamese and Aotearoa-New Zealand educational environments in relation to teaching and learning styles, heavy workload, referencing requirements, and class discussions were reported. These problems left them feeling overwhelmed and bewildered.

The students encountered more difficulties in everyday than academic settings. It has been suggested that international students from some Asian countries with predominant collectivist cultures living in Western countries with individualist cultures may be particularly prone to undesirable experiences during the process of settling in a new country (Khawaja \& Stallman, 2011). The students emphasised that they got accustomed to the new education environment after the first trimester. For example, they could deal effectively with issues related to referencing after receiving their lecturers' feedback. Difficulties with understanding assignments' criteria or lecturers' expectations for assignments usually diminished after the first assignment. However, issues related to living arrangements remained after a year. A number of them changed accommodation two or three times during their time in Aotearoa-New Zealand, and it was likely that this would continue happening if they remained in the country after completing their degrees. Besides, a strong 
connection with family was associated with a sense of not belonging after their nearly a year in the country. These findings about the students' living and learning experiences showed that they were active agentic beings who managed to become independent through either overcoming difficulties or deciding to live with them.

\section{Theoretical Contributions of the Study and Reconceptualisation of the Theoretical Model}

My research contributes to the local and international literature by exploring the individual experiences of the Vietnamese international students in New Zealand. I emphasised the role agency played in their experiences. Conceptualised as agentic beings, international students are capable of deciding on what they wish to do and who they want to be. International student security was crucial because it recognised students were bearers of rights, just like domestic students, and were protected in host countries. My revamped conceptual model is presented in Figure 10.1. 


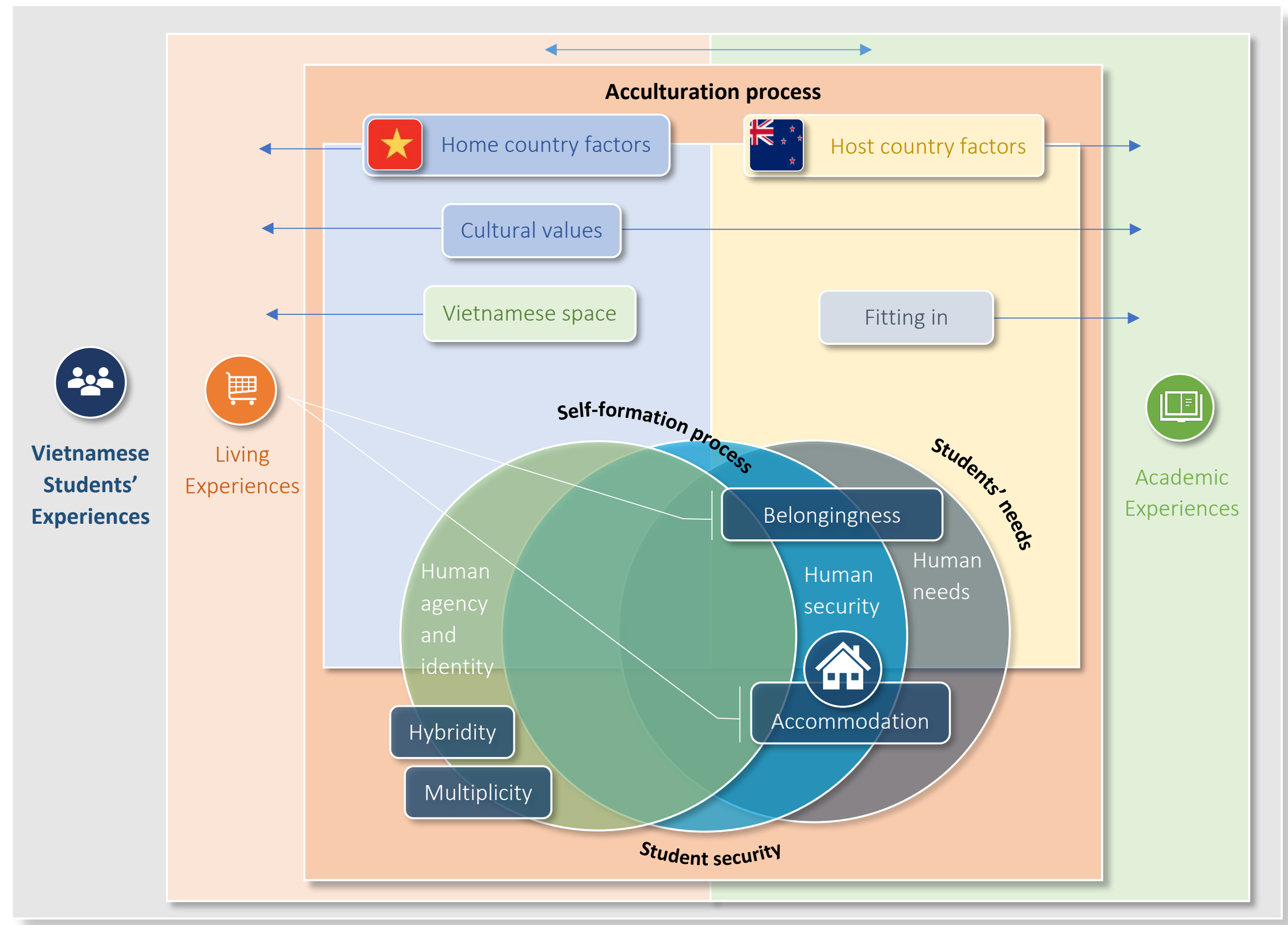

Figure 10.1. Acculturation framework for short-term Vietnamese international students 
In relation to human needs and security, housing issues emerged as an important contributor to international students' experiences, which is a neglected area of research. Past research has focused on the role of language, academic, social and emotional factors. Issues related to accommodation have only been brought to attention in recent years. This study has underlined the need to understand this aspect of their experiences.

This study has shown the limitations of Berry's acculturation framework and sheds light on the way in which the circumstantial conditions precluded the students from making their preferred choices. In a number of conditions (e.g., time constraints and academic pressure and priority), their ability to exercise agency was limited. Such conditions make their experiences unique and different from those of other cultural travellers, for example, immigrants and businesspeople. Also, these findings suggest that short-term international students' experiences are likely to be different from that of long-term students.

Rather than attempting to fit the students' experiences into the acculturation model for immigrants, I made it more relevant to their unique situations by incorporating other theories. The theoretical framework, including Berry's acculturation framework, Marginson and colleagues' perceptions of international student security, Marginson's concept of student self-formation, and Maslow's hierarchy of needs, facilitated the interdisciplinary approach of this study. This approach has advantages because the theories complemented each other, offered a broader and more detailed interpretative lens, and enabled me to look at and understand the investigated issues from multiple perspectives.

Based on the findings, I revamped the conceptual model proposed in Chapter Two to make it more relevant to Vietnamese international students. Belongingness resulted from various daily life issues and emerged as a significant contributor to the students' experiences. Vietnamese cultural values, which were part of home country factors, had a considerable impact on their experiences. I added 'accommodation' to the model to indicate the importance of these factors. Based on the findings, this new element of 'accommodation' was conceptualised as being relevant to the students' needs and security.

As discussed in Chapter Nine, the students might employ different 'acculturation strategies' in different contexts. Berry's concepts of four acculturation strategies have largely failed to 
reflect the complexity of the students' engagement with host country cultures and communities in different contexts. Considering such mismatches between Berry's conceptualisation and the students' experiences, I propose new terms that better capture their choices of strategies. In everyday settings, the students tended to live exclusively with co-nationals, which helped them establish a 'small community' within their houses and feel safe in an unfamiliar environment. They jointly created a 'temporary home', where they could be themselves and live 'Vietnamese lives' through communicating in their mother tongue, cooking Vietnamese foods, and celebrating traditional holidays. I conceptualised these 'homes' as 'Vietnamese space'. In academic settings, rather than adopting assimilation strategies, the students wanted to absorb new academic values and practices, and 'fit in' the academic environment. I had no evidence for marginalisation, and thus removed it from the initial model.

The students transformed through their education and intercultural experiences. There was some evidence that they formed new hybrid or multiple identities (Marginson, 2014). Marginson's ideas of self-formation, hybridity and multiplicity strategies and Berry's integration strategy are different but related to each other. In adapting to the new environments, these students made choices as to what strategies to use in engaging with the host country's communities and cultures. Hybridity and multiplicity involve their constructing and reconstructing their own identities within the Aotearoa-New Zealand context. They treasured Vietnamese cultural values and identities and were interested in the host country's practices and values. They decided whether to 'organise' their host and home country identities as separate parts (multiplicity), to combine them to form a larger whole (hybridity), or to use both depending on the context. Their active agency was shown through (re)constructing and negotiating their home and host identities and switching between them. By taking advantage of hybridity and multiplicity strategies, the students engaged in the process of self-formation.

Marginson's ideas of international student self-formation with a focus on hybridity and multiplicity supported my findings which highlighted the students' agency and identity. Therefore, rather than using the term 'integration', I employed the terms 'hybridity' and 'multiplicity' to highlight their transformation in both everyday and academic settings. 


\section{Strengths and Limitations}

There are strengths and limitations of the current study which are related to methodology. In this section, I address its strengths first, then move on to its limitations.

\section{Strengths}

To date, few studies have explored the experiences of Vietnamese students in AotearoaNew Zealand, especially in everyday settings. My study offers insights into an important aspect of their experiences which are related to housing and living arrangements. By focusing on these aspects, the study has offered a rich account of what it was like to be a Vietnamese student in Aotearoa-New Zealand. It has shown how issues in living arrangements might affect their learning experience and how they dealt with problems.

Most previous research on Vietnamese international students has been survey-based, standard case studies, or employed narrative inquiry. A relatively homogenous group of participants has allowed me to identify and capture the nuanced aspects and the shared essence of individual experiences within a particular group. A small sample enabled me to obtain rich and in-depth accounts of their experiences and understand how they made sense of the experiences.

The phenomenological research design with semi-structured interviews and analysis procedures allowed me to focus on new themes and topics emerging in the process of data collection or analysis. During the research process, I attempted to engage in the doublehermeneutic - a multi-layered analysis of their experiences, in which I endeavoured to make sense of the students trying to make sense of their experiences. The hermeneutic circle which emphasises the critical relationships between the parts and the whole of what was emerging enabled me to unpack the complexity of their experiences.

Rather than adopting the adjustment paradigm in psychology that has been widely used in previous studies on international students' experiences (Ra \& Trusty, 2017; Szabó et al., 2020) and that has been criticised as potentially emphasising cultural superiority and students' abandonment of their home country identities (Marginson, 2014), my project takes a student-centred perspective, and highlights the students' agency and identity. 
Given the purpose of my study is to understand how the students made sense of their experiences and capture the essence of those experiences, I consider the use of IPA as an appropriate choice. The above discussion has shown that there is a range of strengths coming from the use of IPA. However, it is important to recognise its limitations, which are presented in the next section.

\section{Limitations}

The sample of this study was small and comprised 10 Vietnamese students at a university in Wellington, Aotearoa-New Zealand, and was from three disciplines. These affected the degree to which the findings can be transferred to other settings. I described the relevant research contexts that were central to the study to aid other researchers' judgement of the transferability. Due to the constraints of the project, I was unable to interview the students before they came to New Zealand or after they returned to Vietnam. I did not observe the students in their classes. The participants might feel uncomfortable when talking about some potentially important aspects of their experiences (e.g., romantic relationship) because of my gender and my age. Besides, the participants lived in private accommodation and mainly lived with Vietnamese people, which restricts the study's possibility to reflect the living experiences of students living in other types of housing and living with people from other countries. There were only three male students out of ten, which made it difficult to capture the impacts of gender differences in their experiences. Based on these limitations, I present suggestions for further research in the next section.

\section{Suggestions for Future Research}

The findings show that the students' experiences with housing and living arrangements were important and complex. Future studies could employ a different research design (e.g., survey or mixed methods), using a larger sample of international students lodging in other types of accommodation and coming from other countries, looking at how they deal with housing-related issues.

Besides interviews, observing participants in the settings can help IPA researchers gather rich data about their experiences. Doctoral students tend to stay in host countries for a 
longer period than master's students and as a result, they may have different experiences. Being accompanied by their families in host countries might significantly affect students' adjustment experiences. In the light of these considerations, it is worth focusing on these student cohorts.

\section{Implications for Universities and Students}

Globally, more people than ever before are choosing to undertake higher or further education outside their home countries. International students now form a substantial proportion of the diverse student community at many Western universities, including VUW. Apart from differences in cultural values and beliefs, ethnic, educational backgrounds, there are similarities among many international students. For example, they move to a foreign country with a different culture and attend universities with different teaching and learning environments. These factors are interwoven with each other and contribute to the complexity of their experiences. This requires academics and universities to take measures to accommodate their diverse needs.

The findings have implications for universities and (prospective) international students. The Vietnamese students face difficulties concerning living arrangements. In the context where local and international students are challenged in finding suitable accommodation, institutions ideally would provide greater help in navigating the local housing market and securing accommodation. When the participants were not able to reside within the university halls, they turned to other housing options, one of which was the local private rental sector. Here the issue might become complex because it involved stakeholders, such as landlords and property agents. There is a need for institutions to proactively work with students and property agents and support students with navigating unfamiliar and challenging rental markets. There was limited evidence from the students' narratives that the university was providing them with such necessary advice and support. With these students, it appeared that the institution provided cursory information and left them to find accommodation. This points to a need that rather than just providing details on websites and expecting students to be responsible for investigating such information, education providers take measures to ensure that pastoral care is extended to students. 
It may be beneficial for students if agents and universities in both home and host countries could find out what support and pre-arrival assistance they need, help to find suitable accommodation, and make housing information accessible and related to their needs. Host universities may consider taking measures to increase the availability of affordable quality accommodation.

For the students, living with people outside of their families and dealing with landlords were new and challenging experiences. Other international students may have similar experiences. Universities could consider providing students with practical 'tips' on how to get along with their flatmates and to negotiate with others such as landlords.

It would be important for prospective international students to be well prepared for their new lives in foreign countries. They should actively research conditions in the new country and seek guidance from co-nationals who have studied in the host country. It would be useful for international students to equip themselves with knowledge about the social and cultural norms and values of the host country before they go abroad. Good preparation for overseas study, including language, knowledge of the host country, campus life, academic environment, can facilitate students' academic success.

Improving English should be a shared responsibility of students and institutions (Andrade \& Hartshorn, 2019). It is recommended that students be aware of their language ability and attempt to improve it. Educational institutions need to consider how to help students cope with language issues through curricular and cocurricular.

\section{Researcher's Reflection: "The End of One Journey Marks the Beginning of Another." - Luke Davies}

After a journey, I often sit down and reflect on what issues I encountered on the way. Research not only is about an outcome, but also a journey. Before conducting this research, I was heavily influenced by my experience as an international student. My fairly rudimentary understanding of what it would be like to be a Vietnamese student in New Zealand was gradually altered through the course of the research process. 
I was on a scholarship, had limited prior overseas experiences, and am single. At the beginning of the research process, I simply assumed that the overseas study would be a challenging yet rewarding journey. Personally, studying and living in a culturally diverse country were like my dream has come true. Although I understood that life and studies in a foreign country could be difficult and demanding, I did not seriously think about the emotional aspect of their experiences. I simply thought the students might find their lives and studies in a new environment stressful but assumed this would not have a strong impact on them. As a male student, I was aware that female counterparts potentially had different experiences, but I knew little about what such differences might be. I had never thought about employing an approach developed by researchers in the field of psychology to explore students' experiences. My initial research proposal was to study international students' experiences using a mixed-methods approach.

When my research progressed, and I interacted more with the participants, listened to their stories, immersed myself in their narratives, and began to analyse the data and discuss with my supervisors, I was gradually aware that there was something amiss with my initial assumptions. There were more emotional elements in their stories than I had assumed. The students felt bewildered, lonely, overwhelmed, and homesick. There were feelings of happiness and confidence when they managed to construct or reconstruct their identities. It took me months to get enlightened. I believe that I have gained a better understanding of their experiences, which would be useful for my future research.

Carrying out this research was particularly difficult for me as initially a novice researcher. Although I had prepared carefully before each interview, I still worried when going into the field. After completing an interview, I often wondered if I did something inappropriately, asked questions that I should not ask, or missed something that I should have discussed. Despite these concerns, I obtained rich data thanks to the openness and cooperation of the students.

Despite such challenges, the research process was rewarding. Through handling interview issues, my interviewing skills were enhanced. I became more confident with the later interviews. What I learned from the research process would be useful to my future research. 
Dealing with a huge amount of data was challenging and during the analysis process, I was often concerned that I was not interpretive enough. However, managing to find my way through it gave me a sense of achievement. I now feel that I have been better equipped as a researcher. Considering that I was keen to utilise a mixed-methods approach in my initial proposal, I was surprised that I developed an interest in qualitative research methods in the course of conducting the study.

Reflecting on my own experiences, I see myself in the participants' stories. We embarked on distinctive journeys but embraced our experiences in pretty much the same way, be they pleasant or not, because they have helped us explore ourselves and (re)construct our identities. In that process, we recognise what we treasure and acknowledging what we needed to improve was just as important as knowing what we were able to do well. Nearing the end of my journey, I wondered if our journeys had drawn us a few steps closer to our 'self-actualisation'. I thought deeply about this. Whatever way it may turn out to be, in a positive and optimistic manner, I do hope it had.

Every journey has an end. I had a deep sense of accomplishment when completing this study. I learnt a lot from working on this thesis, and the experience and knowledge gained are invaluable. However, at some point along the way, I spoke to myself that I needed to finish this study off and to start to work on something else. To me, this is certainly a big project and a huge milestone in my academic life but should not be the only one in my entire career. I look forward to returning to my work in Vietnam and getting involved in new and bigger projects. Apart from that, I am excited to return to my home which I am emotionally attached to and where I feel belonging. 


\section{References}

Adler, L. L., \& Gielen, U. P. (2003). Migration: Immigration and emigration in international perspective. Westport: Praeger Publishers/Greenwood Publishing Group, Inc.

Al-Amer, R., Ramjan, L., Glew, P., Darwish, M., \& Salamonson, Y. (2016). Language translation challenges with Arabic speakers participating in qualitative research studies. International Journal of Nursing Studies, 54, 150-157.

Al-Amer, R., Ramjan, L., Glew, P., Darwish, M., \& Salamonson, Y. (2014). Translation of interviews from a source language to a target language: Examining issues in crosscultural health care research. Journal of Clinical Nursing, 24, 1151-1162. doi:https://doi.org/10.1111/jocn.12681

Alexander, P. A. (2005). Psychology in learning and instruction. Englewood Cliffs, NJ: Prentice Hall.

Alloh, F., Tait, D., \& Taylor, C. (2018). Away from home: A qualitative exploration of health experiences of Nigerian students in a UK university. Journal of International Students, 8(1), 1-20.

Alsahafi, N., \& Shin, S.-C. (2017). Factors affecting the academic and cultural adjustment of Saudi international students in Australian universities. Journal of International Students, 7(1), 53-72.

Altbach, P. G. (1998). Comparative higher education: Knowledge, the university, and development. Hong Kong: Comparative education research centre, the university of Hong Kong.

Ammigan, R. (2019). Institutional satisfaction and recommendation: What really matters to international students. Journal of International Students, 9(1), 262-281.

An, S., Lim, S. S., Lee, H., \& Pham, B. (2018). Overcoming prejudice, seeking support: Transnational social media communication of female Vietnamese students in South Korea and Singapore. Transitions: Journal of Transient Migration, 2(2), 107-126.

Andrade, M. S. (2006). International students in English-speaking universities: Adjustment factors. Journal of Research in International Education, 5(2), 131-154.

Andrade, M. S., \& Hartshorn, K. J. (2019). International student transitions: A framework for success. Newcastle, UK: Cambridge Scholars Publishing.

Argyle, M. (1969). Social interaction. London: Methuen.

Arkoudis, S., Dollinger, M., Baik, C., \& Patience, A. (2019). International students' experience in Australian higher education: Can we do better? Higher Education, 77(5), 799-813.

Australian Education International. (2020). Monthly summary of international student data - Australia - March $2020 . \quad$ Retrieved from https://internationaleducation.gov.au/research/International-StudentData/Documents/MONTHLY\%20SUMMARIES/2020/Mar\%202020\%20MonthlyInfog raphic.pdf 
Avolonto, A. C. (2020). The choice of acculturation strategies: Intercultural adaptation of French international students from Sub-Saharan African Francophone countries in Ontario. (Unpublished master's thesis), York University, Retrieved from https://yorkspace.library.yorku.ca/xmlui/bitstream/handle/10315/37479/Coco Av olonto Aurore 2019 Masters.pdf? sequence=2\&isAllowed=y

Bartram, B. (2008). Supporting international students in higher education: Constructions, cultures and clashes. Teaching in Higher Education, 13(6), 657-668.

Belford, N. (2017). International students from Melbourne describing their cross-cultural transitions experiences: Culture shock, social interaction, and friendship development. Journal of International Students, 7(3), 499-521.

Benner, P. (1994). Interpretive phenomenology: Embodiment, caring, and ethics in health and illness. Thousand Oaks, CA: Sage.

Berry, J. W. (1997). Immigration, acculturation, and adaptation. Applied Psychology, 46(1), 5-34.

Berry, J. W. (2006). Stress perspectives on acculturation. In D. L. Sam \& J. W. Berry (Eds.), The Cambridge handbook of acculturation psychology (pp. 43-57). Cambridge, UK: Cambridge University Press.

Berry, J. W. (2009). A critique of critical acculturation. International Journal of Intercultural Relations, 33(5), 361-371. doi:https://doi.org/10.1016/j.ijintrel.2009.06.003

Berry, J. W. (2017). Theories and models of acculturation. In S. J. Schwartz \& J. B. Unger (Eds.), Oxford handbook of acculturation and health (pp. 15-27). New York: Oxford University Press.

Berry, J. W., Kim, U., Minde, T., \& Mok, D. (1987). Comparative studies of acculturative stress. The International Migration Review, 21(3), 491-511. doi:https://doi.org/10.2307/2546607

Berry, J. W., \& Sam, D. L. (2016). Theoretical perspectives. In D. L. Sam \& J. W. Berry (Eds.), The Cambridge handbook of acculturation psychology. Cambridge, UK: Cambridge University Press.

Berry, J. W., Trimble, J. E., \& Olmedo, E. L. (1986). The assessment of acculturation. In W. J. Lonner \& J. W. Berry (Eds.), Field methods in cross-cultural research (pp. 291-324). Newbury Park, CA: Sage.

Bhowmik, M. K., Cheung, R. Y., \& Hue, M. T. (2018). Acculturative stress and coping strategies among Mainland Chinese university students in Hong Kong: A qualitative inquiry. American Journal of Orthopsychiatry, 88(5), 550-562.

Biesta, G., Priestley, M., \& Robinson, S. (2015). The role of beliefs in teacher agency. Teachers and Teaching, 21(6), 624-640.

Biesta, G., \& Tedder, M. (2007). Agency and learning in the lifecourse: Towards an ecological perspective. Studies in the Education of Adults, 39(2), 132-149.

Billedo, C. J., Kerkhof, P., Finkenauer, C., \& Ganzeboom, H. (2019). Facebook and face-toface: Examining the short-and long-term reciprocal effects of interactions, perceived 
social support, and depression among international students. Journal of ComputerMediated Communication, 24(2), 73-89.

BMI. (2020). The outbound student recruitment market in Vietnam. Retrieved from https://bmiglobaled.com/Market-Reports/Vietnam/student-recruitment

Bochner, S., Hutnik, N., \& Furnham, A. (1985). The friendship patterns of overseas and host students in an Oxford student residence. The Journal of Social Psychology, 125(6), 689-694.

Bochner, S., McLeod, B. M., \& Lin, A. (1977). Friendship patterns of overseas students: A functional model. International Journal of Psychology, 12(4), 277-294.

Brennan, T. (1982). Loneliness at adolescent. In L. A. Peplau \& D. Perlman (Eds.), Loneliness: A sourcebook of current theory research and therapy (pp. 269-290). New York: John Wiley.

Brisset, C., Safdar, S., Lewis, J. R., \& Sabatier, C. (2010). Psychological and sociocultural adaptation of university students in France: The case of Vietnamese international students. International Journal of Intercultural Relations, 34(4), 413-426. doi:https://doi.org/10.1016/j.ijintrel.2010.02.009

Brocki, J. M., \& Wearden, A. J. (2006). A critical evaluation of the use of interpretative phenomenological analysis (IPA) in health psychology. Psychology and Health, 21(1), 87-108.

Brown, L. (2009). An ethnographic study of the friendship patterns of international students in England: An attempt to recreate home through conational interaction. International Journal of Educational Research, 48(3), 184-193.

Brunsting, N. C., Zachry, C., \& Takeuchi, R. (2018). Predictors of undergraduate international student psychosocial adjustment to US universities: A systematic review from 20092018. International Journal of Intercultural Relations, 66, 22-33.

Bui, T. A. (2021). Becoming an intercultural doctoral student: Negotiating cultural differences. Journal of International Students, 11(1), 257-265. doi:https://doi.org/10.32674/jis.v11i1.1272

Bui, T. H. L. (2017). An investigation of Vietnamese postgraduate students' negotiation of social and academic identities at a UK university. (Unpublished PhD thesis), University of Southampton, England.

Butcher, A., \& McGrath, T. (2011). A sin of omission: New Zealand's export education industry and foreign policy. In C. Holden, M. Kilkey, \& G. Ramia (Eds.), Social policy review 23: Analysis and debate in social policy (Vol. 23, pp. 257-280). Bristol: Policy Press.

Butcher, A., McGrath, T., \& Stock, P. (2008). Once returned, twice forgotten? Asian students returning home after studying in New Zealand. New Zealand Population Review, 33(34), 235-248.

Calder, M. J., Richter, S., Mao, Y., Burns, K. K., Mogale, R. S., \& Danko, M. (2016). International students attending Canadian universities: Their experiences with 
housing, finances, and other issues. The Canadian Journal of Higher Education, 46(2), 92-110.

Campbell, N. (2012). Promoting intercultural contact on campus: A project to connect and engage international and host students. Journal of Studies in International Education, 16(3), 205-227.

Cao, L., \& Zhang, T. (2012). Social networking sites and educational adaptation in higher education: A case study of Chinese international students in New Zealand. The Scientific World Journal, 2012, 1-5. doi:https://doi.org/10.1100/2012/289356

Chapman, E., \& Smith, J. A. (2002). Interpretative phenomenological analysis and the new genetics. Journal of Health Psychology, 7(2), 125-130.

Chase, S. E. (2000). Narrative inquiry: Multiple lenses, approaches, voices. In N. Denzin \& Y. Lincoln (Eds.), Handbook of qualitative research (2nd ed., pp. 651-679). Thousand Oaks, CA: Sage.

Chen, K. (2014). An investigation of cultural factors that influence Chinese accounting students in New Zealand. (Unpublished master's thesis), Auckland University of Technology, New Zealand.

Chen, M., \& Bang, H. (2020). Exploring East Asian undergraduate students' perceptions about the effectiveness of their preparation for study abroad for academic success in U.S. universities. Journal of International Students, 10(1), 181-202.

Chien, Y. G. (2020). Studying abroad in Britain: Advantages and disadvantages. Journal of Research in International Education, 19(1), 69-83.

Coffey, J., Farivar, F., \& Cameron, R. (2018). The job seeking experiences of international graduates in the host country: Australia's lost opportunity? The International Journal of Human Resource Management, 32(4), 846-870. doi:https://doi.org/10.1080/09585192.2018.1504106

Cohen, M. Z., \& Omery, A. (1994). Schools of phenomenology: Implications for research. In J. M. Morse (Ed.), Critical issues in qualitative research methods (pp. 136-156). Thousand Oaks, CA: Sage.

Cowley, P., \& Hyams-Ssekasi, D. (2018). Motivation, induction, and challenge: Examining the initial phase of international students' educational sojourn. Journal of International Students, 8(1), 109-130.

Creswell, J. W. (2014). Research design: Qualitative, quantitative, and mixed methods approaches. Thousand Oaks, CA: Sage.

Creswell, J. W., \& Poth, C. N. (2017). Qualitative inquiry and research design: Choosing among five approaches. Thousand Oaks, CA: Sage.

Cục hợp tác quốc tế - International cooperation department. (2020). Chương trình Học bổng Phát triển New Zealand (New Zealand Scholarships) năm 2021. Retrieved from http://icd.edu.vn/372/chuong-trinh-hoc-bong-phat-trien-new-zealand-newzealand-scholarships-nam-2021.html/BPF/vi-VN/CMS Cat/Cac-hoc-bong-ngoaingan-sach/CMS Detail/1679 
Cultural Atlas. (2020). Vietnamese culture. Retrieved from https://culturalatlas.sbs.com.au/vietnamese-culture/vietnamese-culture-coreconcepts

Dallmayr, F. (2009). Hermeneutics and inter-cultural dialog: Linking theory and practice. Ethics \& Global Politics, 2(1), 23-39.

Dang, X. T., \& Tran, L. T. (2017). From 'somebody'to 'nobody': International doctoral students' perspectives of home-host connectedness. In L. T. Tran \& C. Gomes (Eds.), International student connectedness and identity (pp. 75-91). Singapore: Springer.

Dao, M. T. (2005). An investigation into library use by Vietnamese students at Victoria University of Wellington. (Unpublished master's thesis), Victoria University of Wellington, New Zealand.

Das, A. K., Nguyen, Q. T., Nguyen, A. T., Nomikoudis, M., \& Dung, H. (2019). Course redesign to incorporate flipped delivery: A business degree case in Vietnam. Issues in Educational Research, 29(2), 363-383.

De Jong, G. F., \& Fawcett, J. T. (1981). Motivations for migration: An assessment and a valueexpectancy research model. In G. F. D. Jong \& R. W. Gardner (Eds.), Migration decision-making: Multidisciplinary approaches to microlevel studies in developed and developing countries (pp. 13-58). New York: Pergamon Press.

Deloitte. (2008). The experiences of international students in New Zealand: Report on the results of the national survey 2007. Wellington, New Zealand: Ministry of Education.

Demes, K. A., \& Geeraert, N. (2014). Measures matter: Scales for adaptation, cultural distance, and acculturation orientation revisited. Journal of Cross-Cultural Psychology, 45(1), 91-109.

Dentakos, S., Wintre, M., Chavoshi, S., \& Wright, L. (2017). Acculturation motivation in international student adjustment and permanent residency intentions: A mixedmethods approach. Emerging Adulthood, 5(1), 27-41.

Denzin, N. K., \& Lincoln, Y. S. (2011). Introduction: The discipline and practice of qualitative research (4th ed.). Thousand Oaks, CA: Sage.

Deumert, A., Marginson, S., Nyland, C., Ramia, G., \& Sawir, E. (2005). Global migration and social protection rights: The social and economic security of cross-border students in Australia. Global Social Policy, 5(3), 329-352.

Di Fabio, A., \& Tsuda, A. (2018). The psychology of harmony and harmonization: Advancing the perspectives for the psychology of sustainability and sustainable development. Sustainability, 10(12), 1-15. doi:https://doi.org/10.3390/su10124726

Dilthey, W. (1985). Poetry and experience - Selected works (Vol. 5). Princeton, NJ: Princeton University Press.

Ding, Q. (2016). Understanding Chinese international doctoral students in NewZealand: A literature review of contemporary writings about Chinese overseas research students. New Zealand Journal of Teachers' Work, 13(2), 118-133. 
Ding, Q., \& Devine, N. (2017). Agency and social capital in Chinese international doctoral students' conversion to Christianity. Educational Philosophy and Theory, 49(12), 1161-1172.

Ding, Q., \& Devine, N. (2018). Exploring the supervision experiences of Chinese overseas Phd students in New Zealand. Knowledge Cultures, 6(1), 62-78.

Do, H. T. (2007). East meets West: The adaptation of Vietnamese international students to California community colleges. Journal of Southeast Asian American Education and Advancement, 2(1), 1-30.

Doan, D. H. (2005). Moral education or political education in the Vietnamese educational system? Journal of Moral Education, 34(4), 451-463.

Doan, T. (2012). Vietnamese students' view on plagiarism in the Australian context. Internet Journal of Language, Culture and Society, 34(3), 26-32.

Dobson, J. E., Campbell, N. J., \& Dobson, R. (1987). Relationships among loneliness, perceptions of school, and grade point averages of high school juniors. The School Counselor, 35(2), 143-148.

Douglas, S. R. (2019). Counterfactual understandings: What Japanese undergraduate students wish they had known before a short-term study abroad experience. TESLEJ, 23(4), 1-19.

Dowling, M. (2007). From Husserl to van Manen. A review of different phenomenological approaches. International Journal of Nursing Studies, 44(1), 131-142.

Dowling, M., \& Cooney, A. (2012). Research approaches related to phenomenology: Negotiating a complex landscape. Nurse Researcher, 20(2), 21-27.

Doyle, S. (2016). Changing flows and directions for international education and mobilities in selected Asian countries: Turning to look at Asia from a New Zealand perspective. New Zealand Journal of Asian Studies, 18(2), 13-28.

Doyle, S., Manathunga, C., Prinsen, G., Tallon, R., \& Cornforth, S. (2017). African international doctoral students in New Zealand: Englishes, doctoral writing and intercultural supervision. Higher Education Research \& Development, 37(1), 1-14.

Dudgeon, P., Darlaston-Jones, D., Nikora, L. W., Waitoki, W., Pe-Pua, R., Tran, L. N., \& Rouhani, L. (2016). Changing the acculturation conversation: Indigenous cultural reclamation in Australia and Aotearoa/New Zealand. In D. L. Sam \& J. W. Berry (Eds.), The Cambridge handbook of acculturation psychology (2nd ed.). Cambridge, UK: Cambridge University Press.

Duru, E., \& Poyrazli, S. (2011). Perceived discrimination, social connectedness, and other predictors of adjustment difficulties among Turkish international students. International Journal of Psychology, 46(6), 446-454.

Eatough, V., \& Smith, J. A. (2017). Interpretative phenomenological analysis. In C. Willig \& W. Stainton-Rogers (Eds.), The Sage handbook of qualitative research in psychology (pp. 193-211). London: Sage. 
Education New Zealand. (2018a). International education strategy - He rautaki matauranga a ao 2018-2030. Retrieved from https://enz.govt.nz/assets/Uploads/InternationalEducation-Strategy-2018-2030.pdf

Education New Zealand. (2018b). New Zealand - Vietnam strategic engagement plan on education. Retrieved from https://enz.govt.nz/assets/Uploads/New-Zealand-VietNam-Strategic-Engagement-Plan-on-Education-dated-20-February-2018.pdf

Elturki, E., Liu, Y., Hjeltness, J., \& Hellmann, K. (2019). Needs, expectations, and experiences of international students in pathway programs in the United States. Journal of International Students, 9(1), 192-210.

Emirbayer, M., \& Mische, A. (1998). What is agency? American journal of sociology, 103(4), 962-1023.

Fallatah, R. H. M., \& Syed, J. (2018). A critical review of Maslow's hierarchy of needs. In Employee motivation in Saudi Arabia (pp. 19-59). Cham: Palgrave Macmillan.

Farbenblum, B., Nawaz, M., Barton, C., Djasmeini, J., Farrell, H., Meikle, P., ... Wang, W. (2019). No place like home: Addressing exploitation of international students in Sydney's housing market. Retrieved from https://apo.org.au/sites/default/files/resource-files/2019-07/apo-nid246611 2.pdf

Financial Times. (2020). US says foreign students must leave if classes go fully online. Retrieved from https://www.ft.com/content/562bfeec-0955-4689-911b$\underline{\text { b5137c4ba6b5 }}$

Fincher, R., Carter, P., Tombesi, P., Shaw, K., \& Martel, A. (2009). Transnational and temporary: Students, community and place-making in central Melbourne. Melbourne: University of Melbourne.

Fleming, V., Gaidys, U., \& Robb, Y. (2003). Hermeneutic research in nursing: Developing a Gadamerian-based research method. Nursing Inquiry, 10(2), 113-120.

Folkman, S. (2008). The case for positive emotions in the stress process. Anxiety, Stress, and Coping, 21(1), 3-14. doi:https://doi.org/10.1080/10615800701740457

Forbes-Mewett, H. (2018). The new security: Individual, community and cultural experiences. London: Palgrave Macmillan.

Forbes-Mewett, H., Marginson, S., Nyland, C., Ramia, G., \& Sawir, E. (2009). Australian university international student finances. Higher Education Policy, 22(2), 141-161.

Forbes-Mewett, H., \& Sawyer, A.-M. (2016). International students and mental health. Journal of International Students, 6(3), 661-677.

Franken, M. (2012). Re-situation challenges for international students 'becoming' researchers. Higher Education, 64(6), 845-859.

Franken, M. (2013). Significant knowledge transitions and resituation challenges in becoming a researcher: International scholarship students' perspectives. International Journal for Researcher Development, 4(2), 86-102.

Furnham, A. (2019). Culture shock: A review of the literature for practitioners. Psychology, 10(13), 1832-1855. doi:https://doi.org/10.4236/psych.2019.1013119 
Gambrel, P., \& Cianci, R. (2003). Maslow's hierarchy of needs: Does it apply in a collectivist culture. Journal of Applied Management and Entrepreneurship, 8(2), 143-161.

Gill, M. J. (2014). The possibilities of phenomenology for organizational research. Organizational Research Methods, 17(2), 118-137.

Giorgi, A. (2009). The descriptive phenomenological method in psychology: A modified Husserlian approach. Pennsylvania: Duquesne University Press.

Giorgi, A. (2010). Phenomenology and the practice of science. Existential Analysis: Journal of the Society for Existential Analysis, 21(1), 3-22.

Glass, C. R., \& Westmont, C. M. (2014). Comparative effects of belongingness on the academic success and cross-cultural interactions of domestic and international students. International Journal of Intercultural Relations, 38(1), 106-119.

Gribble, C. (2011). National policies on skilled labour and the cross-border student market, with a focus on Vietnam. In S. Marginson, S. Kaur, \& E. Sawir (Eds.), Higher education in the Asia Pacific: Strategic responses to globalization (pp. 291-307). London, UK: Springer Dordrecht Heidelberg.

Gu, Q., \& Schweisfurth, M. (2015). Transnational connections, competences and identities: Experiences of Chinese international students after their return 'home'. British Educational Research Journal, 41(6), 947-970.

Guba, E. G. (1990). The alternative paradigm dialog. In E. G. Guba (Ed.), The paradigm dialog (pp. 17-30). Newbury Park, CA: Sage.

Hagerty, B. M., \& Patusky, K. (1995). Developing a measure of sense of belonging. Nursing research, 44(1), 9-13.

Harman, G., Hayden, M., \& Pham, T. N. (2010). Higher education in Vietnam: Reform, challenges and priorities. In G. Harman, M. Hayden, \& T. N. Pham (Eds.), Reforming higher education in Vietnam: Challenges and priorities (1st ed., pp. 1-14). Dordrecht: springer.

Harvey, T., Robinson, C., \& Welch, A. (2017). The lived experiences of international students whose family remains at home. Journal of International Students, 7(3), 748-763.

Hefferon, K., \& Gil-Rodriguez, E. (2011). Interpretative phenomenological analysis. The Psychologist, 24(10), 756-759.

Heidegger, M. (1988). The basic problems of phenomenology (A. Hofstadter, Trans. Vol. 478). Bloomington, ID: Indiana University Press.

Heidegger, M. (1996). Being and time (J. Stambaugh, Trans.). Albany, NY: SUNY Press.

Hendrickson, B., Rosen, D., \& Aune, R. K. (2011). An analysis of friendship networks, social connectedness, homesickness, and satisfaction levels of international students. International Journal of Intercultural Relations, 35(3), 281-295.

Heng, T. T. (2017). Voices of Chinese international students in USA colleges: 'I want to tell them that...'. Studies in Higher Education, 42(5), 833-850. 
Heng, T. T. (2018). Different is not deficient: Contradicting stereotypes of Chinese international students in US higher education. Studies in Higher Education, 43(1), 2236.

Hiep, P. H. (2001). Teacher development: A real need for English departments in Vietnam. English Teaching Forum, 39(4), 30-33.

Ho, H. T. (2020). The experiences of Vietnamese students in New Zealand: A new countrya new home. Transitions: Journal of Transient Migration, 4(2), 203-221.

Hoang, M., Moslehpour, M., \& Seitz, V. (2019). Decision making model of Vietnamese students studying higher education in England. IAFOR Journal of Education, 7(2), 131-148.

Hoang, T. V. Y., \& Ma, L. P. F. (2016). Experiences of publishing in English: Vietnamese doctoral students' challenges and strategies. Retrieved from https://wac.colostate.edu/docs/atd/australasia/hoang ma2019.pdf

Hofstede, G. (2011). Dimensionalizing cultures: The Hofstede model in context. Online readings in psychology and culture, 2(1), 8. doi:https://doi.org/10.9707/2307$\underline{0919.1014}$

Huff, A. S. (2009). Designing research for publication. Los Angeles, CA: Sage.

Huong, T. T., \& Cong, L. V. (2018). Motivations of Vietnamese students in study abroad: A case of Vietnamese graduate students in the Philippines. International Research in Education, 6(6), 14-29.

Hussain, M., \& Shen, H. (2019). A study on academic adaptation of international students in China. Higher Education, 9(4), 80-91.

Husserl, E. (1900). Logical investigations (J. N. Findlay, Trans.). NY: Humanities Press.

Husserl, E. (1973). Experience and judgment: Investigations in a genealogy of logic (J. S. Churchill \& K. Ameriks, Trans.). Evanston, IL: Northwestern University Press.

Husserl, E. (2001). The shorter logical investigations (J. N. Findlay, Trans.). London: Routledge.

ICEF Monitor. (2018a). Education now New Zealand's fourth-largest export sector. Retrieved from https://monitor.icef.com/2018/11/education-now-new-zealands-fourthlargest-export-sector/

ICEF Monitor. (2018b). From the field: Vietnam's growing middle class fueling demand for study abroad. Retrieved from https://monitor.icef.com/2018/01/vietnams-growingmiddle-class-fueling-demand-study-abroad/

ICEF Monitor. (2018c). New Zealand's foreign enrolment dipped in 2017. Retrieved from https://monitor.icef.com/2018/05/new-zealands-foreign-enrolment-dipped-2017/

ICEF Monitor. (2019). South Korea reporting big gains in foreign enrolment. Retrieved from https://monitor.icef.com/2019/09/south-korea-reporting-big-gains-in-foreignenrolment/

Idczak, S. E. (2007). I am a nurse: Nursing students learn the art and science of nursing. Nursing Education Perspectives, 28(2), 66-71. 
IIE. (2019). A quick look at global mobility trends. Retrieved from https://www.iie.org/en/Research-and-Insights/Project-Atlas/Explore-

Data/Infographics/2019-Project-Atlas-Infographics

IIE. (2020a). Project atlas - America. Retrieved from https://www.iie.org/Research-andInsights/Project-Atlas/Explore-Data/United-States

IIE. (2020b). Project atlas - Canada. Retrieved from https://www.iie.org/Research-andInsights/Project-Atlas/Explore-Data/Canada

IIE. (2020c). Project Atlas - Japan. Retrieved from https://www.iie.org/Research-andInsights/Project-Atlas/Explore-Data/Japan

IIE. (2020d). Project Atlas - New Zealand. Retrieved from https://www.iie.org/Researchand-Insights/Project-Atlas/Explore-Data/New-Zealand

Ike, N., Baldwin, C., \& Lathouras, A. (2017). Optimising tertiary student accommodation within university neighbourhoods. Articulo-Journal of Urban Research. doi:https://doi.org/10.4000/articulo.3243

Indexmundi. (2019). Vietnam Demographics Profile 2019. Retrieved from https://www.indexmundi.com/vietnam/demographics profile.html

International student ambassadors - University of Tartu. (2016). Study abroad: The untold story. Retrieved from http://isa.ut.ee/blog/study-abroad-the-untold-story-thaivietnam/

Jamaludin, N. L., Sam, D. L., \& Sandal, G. M. (2018). Destination motivation, cultural orientation, and adaptation: International students' destination-loyalty intention. Journal of International Students, 8(1), 38-65.

Japan Student Services Organization. (2019). International Students in Japan 2018. Retrieved from https://www.jasso.go.jp/en/about/statistics/intl student/ icsFiles/afieldfile/2019 104/19/data18 brief e.pdf

Jindal-Snape, D., \& Ingram, R. (2013). Understanding and supporting triple transitions of international doctoral students: ELT and SuReCom models. Journal of Perspectives in Applied Academic Practice, 1(1), 17-24.

Johnson, B., \& Christensen, L. (2014). Educational research: Quantitative, qualitative, and mixed approaches (5th Ed.). Thousand Oaks, CA: Sage.

Judd, S. E. (2013). Housing trajectories of Chinese international students in Sydney, Australia. University of New South Wales, Australia.

Kelly, K. (2000). The higher education system in Vietnam. Institute of International Education/Vietnam, World EducationServices, 13(3), 5-6.

Khanal, J., \& Gaulee, U. (2019). Challenges of international students from pre-departure to post-Study: A literature review. Journal of International Students, 9(2), 560-581.

Khawaja, N. G., \& Stallman, H. M. (2011). Understanding the coping strategies of international students: A qualitative approach. Australian Journal of Guidance and Counselling, 21(2), 203-224. 
Kidman, J., Manathunga, C., \& Cornforth, S. (2017). Intercultural PhD supervision: Exploring the hidden curriculum in a social science faculty doctoral programme. Higher Education Research \& Development, 36(6), 1208-1221.

Kim, C.-J., Park, H.-R., Schlenk, E. A., Kang, S.-W., \& Seo, E. J. (2019). The moderating role of acculturation mode on the relationship between depressive symptoms and healthrelated quality of life among international students in Korea. Archives of Psychiatric Nursing, 33(6), 192-197.

Kim, N., Oh, S., \& Mumbauer, J. (2019). Supporting international students: Enhancing college counselors' multicultural counseling competence. Journal of College Counseling, 22(2), 179-192.

Knight, J. (2018). International education hubs. In P. Meusburger, M. Heffernan, \& L. Suarsana (Eds.), Geographies of the university (pp. 637-655). Cham: Springer Nature.

Kovach, M. (2017). Doing indigenous methodologies: A letter to a research class. In N. Denzin \& Y. Lincoln (Eds.), The Sage handbook of qualitative research (5th ed., pp. 214-234). Thousand Oaks, CA: Sage.

Krsmanovic, M. (2020). "I was new and I was afraid": The acculturation strategies adopted by international first-year undergraduate students in the United States. Journal of International Students, 10(4), 954-975. doi:https://doi.org/10.32674/jis.v10i4.1160

Kudo, K., \& Simkin, K. A. (2003). Intercultural friendship formation: The case of Japanese students at an Australian university. Journal of Intercultural Studies, 24(2), 91-114.

Kukatlapalli, J. (2016). A study of the adjustment experiences of Indian international students in New Zealand universities. (Unpublished doctoral thesis), Victoria University of Welington, New Zealand.

Kukatlapalli, J., Doyle, S., \& Bandyopadhyay, S. (2020). An investigation into the English language experiences of Indian international students studying in New Zealand universities. Higher Education Research \& Development, 39(3), 485-499.

Kuo, Y.-H. (2013). Exploring Asian international graduate students' non-academic challenges at Auburn university. Journal of Interdisciplinary Studies in Education, 1(2), 52-64.

Ladum, A., \& Burkholder, G. J. (2019). Psychological Adaptation of International Students in the Northern Part of Cyprus. Higher Learning Research Communications, 9(1), 1-19.

Lai, S. L., Pham, H.-H., \& Le, A.-V. (2019). Toward sustainable overseas mobility of Vietnamese students: Understanding determinants of attitudinal and behavioral loyalty in students of higher education. Sustainability, 11(2), 383-399. doi:https://doi.org/10.3390/su11020383

Lamberton, G., \& Ashton-Hay, S. (2015). Preparing Chinese international business students for the transition to undergraduate study in Australia. Journal of Research in International Education, 14(2), 155-171.

Langdridge, D. (2007). Phenomenological psychology: Theory, research and method. Harlow: Pearson Education.

Larkin, M., \& Thompson, A. (2012). Interpretative phenomenological analysis. In D. Harper \& A. R. Thompson (Eds.), Qualitative research methods in mental health and 
psychotherapy: A guide for students and practitioners (pp. 99-116). Oxford: John Wiley \& Sons.

Larkin, M., Watts, S., \& Clifton, E. (2006). Giving voice and making sense in interpretative phenomenological analysis. Qualitative Research in Psychology, 3(2), 102-120.

Laverty, S. M. (2003). Hermeneutic phenomenology and phenomenology: A comparison of historical and methodological considerations. International Journal of Qualitative Methods, 2(3), 21-35.

Lazarus, R. S., \& Folkman, S. (1984). Stress, appraisal, and coping New York: Springer.

Le, A. (2014). Vietnamese international student repatriates: An exploratory study. (Unpublished doctoral thesis), University of Nebraska-Lincoln, Lincoln.

Le, A. T., \& LaCost, B. Y. (2017). Vietnamese graduate international student repatriates: Reverse adjustment. Journal of International Students, 7(3), 449-466.

Le, H., Koo, F. K., Arambewela, R., \& Zutshi, A. (2017). Voices of dissent: Unpacking Vietnamese international student experience. International Journal of Educational Management, 31(3), 280-292.

Lee, E. S. (1966). A theory of migration. Demography, 3(1), 47-57.

Lee, H. K., \& Rhee, B. S. (2019). Hybrid internationalization in Korea: A promising development? International Higher Education, 96(2019), 13-15.

Lem, N. C. (2019). Factors affecting the intercultural adaptation process of international students: A case study of Vietnamese postgraduate students at a tertiary institution in Taiwan. VNU Journal of Social Sciences and Humanities, 5(2), 163-175.

Lértora, I., Sullivan, J., \& Croffie, A. (2017). They are here, now what do we do? Recommendations for supporting international student transitions. VISTAS 2017, 112.

Li, B. D. (1993). A brief overview of sino-western exchange: Past and present. In H. B. Ruth Hayhoe (Ed.), Knowledge Across Cultures: Universities East and West (pp. 301-308). Toronto, Canada: OISE Press.

Li, H. (2017). Academic integration of mainland Chinese students in Germany. Social Inclusion, 5(1), 80-92.

Li, H. (2019). Academic Integration of Chinese Students in Finland and Germany: A Comparative Perspective. Frontiers of Education in China, 14(2), 234-256.

Li, H., \& Pitkänen, P. (2018). Understanding the Integration of Mainland Chinese Students: The case of Finland. Nordic Journal of Migration Research, 8(2), 107-115.

Li, J.-B., Vazsonyi, A. T., \& Dou, K. (2018). Is individualism-collectivism associated with selfcontrol? Evidence from Chinese and US samples. PLOS ONE, 13(12), 1-14. doi:https://doi.org/10.1371/journal.pone.0208541

Li, J., Marbley, A. F., Bradley, L. J., \& Lan, W. (2016). Attitudes toward seeking professional counseling services among Chinese international students: Acculturation, ethnic identity, and English proficiency. Journal of Multicultural Counseling and Development, 44(1), 65-76. 
Li, J., Wang, Y., Liu, X., Xu, Y., \& Cui, T. (2018). Academic adaptation among international students from East Asian countries: A consensual qualitative research. Journal of International Students, 8(1), 194-214.

Lillyman, S., \& Bennett, C. (2014). Providing a positive learning experience for international students studying at UK universities: A literature review. Journal of Research in International Education, 13(1), 63-75.

Lin, Y., \& Kingminghae, W. (2014). Social support and loneliness of Chinese international students in Thailand. Journal of Population Social Studies, 22(2), 141-157.

Lincoln, Y. S., Lynham, S. A., \& Guba, E. G. (2018). Paradigmatic controversies, contradictions, and emerging confluences revisited. In N. K. Denzin \& Y. S. Lincoln (Eds.), The Sage handbook of qualitative research (5th ed., pp. 213-263). Thousand Oaks, CA: Sage.

London, J. D. (2010). Globalization and the governance of education in Viet Nam. Asia Pacific Journal of Education, 30(4), 361-379.

Long, L. D. (2014). Does it matter where college students live? Differences in satisfaction and outcomes as a function of students' living arrangement and gender. The Journal of College and University Student Housing, 40(2), 66-85.

Lopez, G. I., Figueroa, M., Connor, S. E., \& Maliski, S. L. (2008). Translation barriers in conducting qualitative research with Spanish speakers. Qualitative Health Research, 18(12), 1729-1737.

Loveridge, J., Doyle, S., \& Faamanatu-Eteuati, N. (2018). Journeys across educational and cultural borders: International postgraduate students with young children. British Journal of Sociology of Education, 39(3), 333-347.

Maharaja, G. (2018). The impact of study abroad on college students' intercultural competence and personal development. International Research and Review, 7(2), 18-41.

Mai, H. N. T., \& Hall, C. (2017). Changing views of teachers and teaching in Vietnam. Teaching Education, 28(3), 244-256.

Malim, T., Birch, A., \& Wadeley, A. (1992). Perspectives in psychology (1st ed.). Hampshire: Macmillan Press.

Marginson, S. (2012). Including the other: Regulation of the human rights of mobile students in a nation-bound world. Higher Education, 63(4), 497-512.

Marginson, S. (2014). Student self-formation in international education. Journal of Studies in International Education, 18(1), 6-22.

Marginson, S., Kaur, S., \& Sawir, E. (2011). Global, local, national in the Asia-Pacific. In S. Marginson, S. Kaur, \& E. Sawir (Eds.), Higher Education in the Asia-Pacific - Strategic Responses to Globalization (pp. 3-34). Dordrecht: Springer.

Marginson, S., Nyland, C., Sawir, E., \& Forbes-Mewett, H. (2010). International student security. Cambridge: Cambridge University Press.

Marginson, S., \& Sawir, E. (2011). Ideas for intercultural education. New York: Palgrave Macmillan. 
Martens, K., \& Starke, P. (2008). Small country, big business? New Zealand as education exporter. Comparative Education, 44(1), 3-19.

Maslow, A. H. (1943). A theory of human motivation. Psychological Review, 50(4), 370-396.

Maslow, A. H. (1954). Motivation and Personality (1st ed.). New York: Harper \& Row.

Maslow, A. H. (1962). Toward a psychology of being. Princeton, NJ, US. Princeton: D. Van Nostrand Company.

Maslow, A. H. (1970). Motivation and personality (2nd ed.). New York: Viking Press.

Maslow, A. H. (1987). Motivation and personality (3rd ed.). Delhi, India: Pearson Education.

Matheson, R., \& Sutcliffe, M. (2018). Belonging and transition: An exploration of International Business Students' postgraduate experience. Innovations in Education and Teaching International, 55(5), 602-610.

Matthews, J. (2000). Violent visions and speechless days: Corporeality and the politics of image. In I. Ang, S. Chalmers, L. Law, \& M. Thomas (Eds.), Alter/Asians: AsianAustralian Identities in Art, Media and Popular Culture. Annandale: Pluto Press.

Matua, G. A., \& Van Der Wal, D. M. (2015). Differentiating between descriptive and interpretive phenomenological research approaches. Nurse Researcher, 22(6), 2227.

Mazzarol, T., \& Soutar, G. N. (2002). "Push-pull" factors influencing international student destination choice. International Journal of Educational Management, 16(2), 82-90.

McFaul, S. (2016). International students' social network: Network mapping to gage friendship formation and student engagement on campus. Journal of International Students, 6(1), 1-13.

McGrath, C., Palmgren, P. J., \& Liljedahl, M. (2019). Twelve tips for conducting qualitative research interviews. Medical teacher, 41(9), 1002-1006.

McLoughlin, C. (2001). Inclusivity and alignment: Principles of pedagogy, task and assessment design for effective cross-cultural online learning. Distance Education, 22(1), 7-29.

McMillan, K. (2016). Relations and Relationships: 40 years of people movements from ASEAN countries to New Zealand. Retrieved from http://www.asianz.org.nz/reports/wpcontent/uploads/2016/06/Relations-and-Relationships.pdf

Meadows, E. (2011). From aid to industry: A history of international education in Australia. In D. Davis \& B. Mackintosh (Eds.), Making a difference - Australian international education (pp. 50-80). Sydney: UNSW Press.

Mertens, D. M. (2014). Research and evaluation in education and psychology: Integrating diversity with quantitative, qualitative, and mixed methods (4 ed.). Thousand Oaks, CA: Sage.

Mesidor, J. K., \& Sly, K. F. (2016). Factors that contribute to the adjustment of international students. Journal of International Students, 6(1), 262-282. 
Miller, R. S. (2012). Understanding the motivation of Vietnamese international students and their higher education experiences in the United States. (Unpublished doctoral thesis), University of North Texas, Texas.

Montgomery, C. (2010). Understanding the international student experience. New York: Palgrave Macmillan.

Moon, C. Y., Zhang, S., Larke, P., \& James, M. (2020). We are not all the same: A qualitative analysis of the nuanced differences between Chinese and South Korean international graduate students' experiences in the United States. Journal of International Students, 10(1), 28-49.

Moran, D. (2000). Introduction to phenomenology. London: Routledge.

Moustakas, C. (1994). Phenomenological research methods. Thousand Oaks, CA: Sage.

Mukminin, A. (2019). Acculturative experiences among Indonesian graduate students in Dutch higher education. Journal of International Students, 9(2), 488-510.

Naylor, R., Chakravarti, S., \& Baik, C. (2018). Expectations and experiences of off-campus PhD students in Australia. Journal of Further and Higher Education, 42(4), 524-538.

Neto, F. (2021). Loneliness among African international students in Portugal. Journal of International Students, 11(2). doi:https://doi.org/10.32674/jis.v11i2.1379

New Zealand Education. (2020). New Zealand and Vietnam celebrate 45 years of bilateral ties. Retrieved from https://enz.govt.nz/news-and-research/ed-news/new-zealandand-vietnam-celebrate-45-years-of-bilateral-ties/

New Zealand Foreign Affairs and Trade - Manatu Aorere. (2020). Joint statement on the Viet Nam and New Zealand strategic partnership - 22 July 2020. Retrieved from https://www.mfat.govt.nz/en/media-and-resources/news/joint-statement-on-theviet-nam-and-new-zealand-strategic-partnership-22-july-2020/

New Zealand history - Nga korero a ipurangi o Aotearoa. (n.d.). Vietnam war. Retrieved from https://nzhistory.govt.nz/war/vietnam-war\#heading6

New Zealand Ministry of Education. (2017). The international student wellbeing strategy. Retrieved from https://www.education.govt.nz/assets/Documents/Ministry/Strategies-andpolicies/internation/StudentWellbeingStrategyJune2017.pdf

New Zealand Qualifications Authority. (2016). Code of practice 2016 - Includes amendments 2019. Retrieved from https://www.nzqa.govt.nz/assets/Providers-andpartners/Code-of-Practice/Code-of-Practice-Amendments-2019.pdf

New Zealand Qualifications Authority. (2020). Student complaints about providers' compliance with the Code of Practice. Retrieved from https://www.nzqa.govt.nz/providers-partners/education-code-of-practice/studentcomplaints/

Nghia, T. L. H. (2015). Factors influencing prospective international students' motivation for overseas study and selection of host countries and institutions: The case of Vietnamese students. Paper presented at the 26th ISANA International Education Association Conference, Melbourne, Australia. 
Nghia, T. L. H., Phuong, P. T. N., \& Huong, T. L. K. (2020). Implementing the student-centred teaching approach in Vietnamese universities: the influence of leadership and management practices on teacher engagement. Educational Studies, 46(2), 188-204.

Nguyen, B. T. T., \& Pennycook, A. (2018). Dancing, Google and fish sauce: Vietnamese students coping with Australian universities. Asia Pacific Journal of Education, 38(4), 457-472.

Nguyen, C., \& Griffin, P. (2010). Factors influencing student achievement in Vietnam. Procedia - Social and Behavioral Sciences, 2(2), 1871-1877.

Nguyen, C. H. (2018). Historical trends of Vietnamese international student mobility. In L. T. Tran \& S. Marginson (Eds.), Internationalisation in Vietnamese higher education (pp. 141-159). Dordrecht: Springer.

Nguyen, C. T. (2011). Impacts of socio-culture on the development of autonomous learning: A lens of Vietnamese context. Journal of Studies in Education, 1(1), 1-10.

Nguyen, H. N. (1998). The Confucian Incursion into Vietnam. In W. H. Slote \& G. A. D. Vos (Eds.), Confucianism and the Family: A Study of Indo-Tibetan Scholasticism (pp. 91104). New York: State University of New York Press.

Nguyen, L. (2018). Becoming professional researchers: An exploration of the experiences of Vietnamese doctoral students in Australia. (Unpublished doctoral thesis), Retrieved from http://hdl.handle.net/11343/219372

Nguyen, M. L. T. (2012). Vietnamese students' transitions in study abroad programs. Australian Journal of Career Development, 21(3), 13-22.

Nguyen, M. N., \& Robertson, M. J. (2020). International students enacting agency in their PhD journey. Teaching in Higher Education, 1-17. doi:https://doi.org/10.1080/13562517.2020.1747423

Nguyen, N. T. (2019). Cultural modalities of Vietnamese higher education. In N. T. Nguyen \& L. T. Tran (Eds.), Reforming Vietnamese higher education: Global forces and local demands (pp. 17-33). Dordrecht: Springer.

Nguyen, N. T., \& Tran, L. T. (2019). Impacts of global forces and local demands on Vietnamese higher education. In N. T. Nguyen \& L. T. Tran (Eds.), Reforming Vietnamese Higher Education: Global forces and local demands (pp. 1-14). Dordrecht: Springer.

Nguyen, N. T. T. (2020). "The dumbest and most miserable, but I'm fitting in": A call for practice of care ethics. Journal of the Texas Tech University Ethics Center, 2(4), 6576.

Nguyen, Q., \& Buckingham, L. (2019). Source-use expectations in assignments: The perceptions and practices of Vietnamese master's students. English for Specific Purposes, 53, 90-103.

Nguyen, Q. T. N. (2016). The Vietnamese values system: A blend of Oriental, Western and Socialist values. International Education Studies, 9(12), 32-40.

Nguyen, T. (2019). A Journey of cross-cultural adaptation: An autoethnography of a Vietnamese graduate student in the American classroom. (Unpublished master's 
thesis), Eastern Illinois University, the U.S.A. Retrieved from https://thekeep.eiu.edu/cgi/viewcontent.cgi?article=5457\&context=theses

Nguyen, T., \& Angelique, H. (2017). Internalized homonegativity, confucianism, and selfesteem at the emergence of an LGBTQ identity in modern Vietnam. Journal of Homosexuality, 64(12), 1617-1631.

Nguyen, T. D. (2013). Tam giáo đồng nguyên và tính đa nguyên trong truyền thống văn hóa Việt Nam (Three Religions and pluralism in Vietnamese traditional culture). Khoa học Xã hội Việt Nam, 66, 35-43.

Nguyen, T. T. M., \& Cao, T. H. P. (2019). An evaluation of the intercultural orientation of secondary English textbooks in Vietnam: How well are students prepared to communicate in global contexts? In V. C. Le, T. M. H. Nguyen, T. T. M. Nguyen, \& R. Bernard (Eds.), Building teacher capacity in English language teaching in Vietnam: Research, policy and practice (pp. 150-165). Oxon, UK: Routledge.

Nguyen, T. T. T. (2019). Language practices across contact zones: The experiences of Vietnamese students in Taiwan. Language and Intercultural Communication, 19(6), 505-519.

Nhat, H. T., Lien, N. T., Tinh, N. T., Hang, N. V. T., \& Trang, N. T. (2018). The development of critical thinking for students in Vietnamese Schools: From policies to practices. American Journal of Educational Research, 6(5), 431-435.

Nogami, Y. (2020). Study abroad, identity, and attitude towards the English language. In M. Konakahara \& K. Tsuchiya (Eds.), English as a lingua franca in Japan: Towards multilingual practices (pp. 157-181). Cham: Springer International Publishing.

Noon, E. J. (2017). An interpretative phenomenological analysis of the barriers to the use of humour in the teaching of childhood studies. Journal of Perspectives in Applied Academic Practice, 5(3), 45-52.

Noon, E. J. (2018). Interpretive phenomenological analysis: An appropriate methodology for educational research? Journal of Perspectives in Applied Academic Practice, 6(1), 7583.

Nyland, C., Forbes-Mewett, H., Marginson, S., Ramia, G., Sawir, E., \& Smith, S. (2009). International student-workers in Australia: A new vulnerable workforce. Journal of Education and Work, 22(1), 1-14.

O'Dwyer, S. (2017). Deflating the 'Confucian Heritage Culture'thesis in intercultural and academic English education. Language, Culture and Curriculum, 30(2), 198-211.

Obeng-Odoom, F. (2012). Far away from home: The housing question and international students in Australia. Journal of Higher Education Policy and Management, 34(2), 201-216.

Osterman, K. F. (2001). Students' need for belonging in the school community. Review of Educational Research, 70(3), 323-367.

Pacheco, E.-M. (2020). International student well-being and the influence of politics. In U. Gaulee, S. Sharma, \& K. Bista (Eds.), Rethinking education across borders (pp. 193207). Singapore: Springer. 
Palmer, Y. (2015). Triple learning: The journey from student to scholar. University of Saskatchewan, Retrieved from https://harvest.usask.ca/handle/10388/ETD-2015$\underline{02-1951}$

Park, H., Lee, M.-J., Choi, G.-Y., \& Zepernick, J. S. (2017). Challenges and coping strategies of East Asian graduate students in the United States. International Social Work, 60(3), 733-749.

Pham, A. (2017). Visualising returnee re-engagement with local workplaces and community: A case study of Ho Chi Minh city, Vietnam. In L. T. Tran \& C. Gomes (Eds.), International student connectedness and identity (pp. 137-150). Singapore: Springer.

Pham, H.-H., \& Lai, S. L. (2016). Higher education as an extended duration service: An investigation of the determinants of Vietnamese overseas student loyalty. Journal of Studies in International Education, 20(5), 454-471.

Pham, H.-H., Lai, S. L., \& Vuong, Q.-H. (2019). The role of subjective task value in forming satisfaction and loyalty among Vietnamese international students: A structural equation model. The Asia-Pacific Education Researcher, 28(5), 399-409.

Pham, H. H. (2018). International students' choice of destinations for overseas study: A specific push-pull model for Vietnam. In L. T. Tran \& S. Marginson (Eds.), Internationalization in Vietnamese Higher Education (pp. 161-175). Dordrecht: Springer.

Pham, L. (2013). Social structures in the economics of international education: Perspectives from Vietnamese international tertiary students. Globalisation, Societies and Education, 11(1), 39-60.

Pham, L., \& Saltmarsh, D. (2013). International students' identities in a globalized world: Narratives from Vietnam. Journal of Research in International Education, 12(2), 129141.

Pham, L. T. (2016). Understanding the transformative potential of international education for Vietnamese overseas graduates and their communities. (Unpublished doctoral thesis), Macquarie University, Australia.

Pham, N. C., \& Shi, J. R. (2020). A qualitative study on mental distress of Vietnamese students in the USA in the Covid-19 era. Asia Pacific Journal of Health Management, 15(3), 45-57.

Pham, T. H. T. (2010). Implementing a student-centered learning approach at Vietnamese higher education institutions: Barriers under layers of casual layered analysis (CLA). Journal of Futures Studies, 15(1), 21-38.

Phan, H. L. (2001). How do culturally situated notions of 'polite'forms influence the way Vietnamese postgraduate students write academic English in Australia? Australian Journal of Education, 45(3), 296-308.

Phan, H. L. (2008). Teaching English as an international language: Identity, resistance and negotiation. Clevedon: Multilingual Matters. 
Pietkiewicz, I., \& Smith, J. A. (2014). A practical guide to using interpretative phenomenological analysis in qualitative research psychology. Psychological Journal, 20(1), 7-14.

Polit, D. F., \& Beck, C. T. (2005). Essentials of nursing research: Methods, appraisal, and utilization (6th ed.). Philadelphia: Lippincott Williams \& Wilkins.

Pond, E. (2014). Vietnam's second revolution. World Policy Journal(Spring issue: Sex and Sexuality). doi:http://dx.doi.org/10.1177/0740277514529723

Poyrazli, S., Arbona, C., Bullington, R., \& Pisecco, S. (2001). Adjustment issues of Turkish college students studying in the United States. College Student Journal, 35(1), 52-52.

Presbitero, A. (2016). Culture shock and reverse culture shock: The moderating role of cultural intelligence in international students' adaptation. International Journal of Intercultural Relations, 53(1), 28-38.

Prime Minister of Australia. (2020). Press conference - Australian parliament house, act. Retrieved from https://www.pm.gov.au/media/press-conference-australianparliament-house-act-030420

Prime Minister of Socialist Republic of Vietnam. (2000). Quyêt định về việc phê duyệt đề án "đào tạo cán bộ khoa học, kỹ thuật tại các cơ sở nước ngoài bằng ngân sách nhà nước" [Approval of the Scheme on "Training of cadres and scientists at foreign establishments by state budget"]. Retrieved from https://thuvienphapluat.vn/vanban/tai-chinh-nha-nuoc/Quyet-dinh-322-2000-QD-TTg-De-an-Dao-tao-can-bokhoa-hoc-ky-thuat-tai-nuoc-ngoai-bang-ngan-sach-nha-nuoc-8543.aspx

Prime Minister of Socialist Republic of Vietnam. (2010). Quyêt định phê duyệt Đề án Đào tạo giảng viên có trình độ tiến sĩ cho các trường đại học, cao đẳng giai đoạn 2010 - 2020 [Approval of the scheme on "Training for Lecturers with Doctoral degree for for Universities and Colleges in the Period 2010-2020"]. Retrieved from http://www.chinhphu.vn/portal/page/portal/chinhphu/hethongvanban?class id=1 \&mode=detail\&document $i d=95252$

Pringle, J., Hendry, C., \& McLafferty, E. (2011). Phenomenological approaches: Challenges and choices. Nurse Researcher, 18(2), 7-18.

Pusch, M. D. (2004). Intercultural training in historical perspective. In D. Landis, J. M. Bennett, \& M. J. Bennett (Eds.), Handbook of Intercultural Training (3rd ed., pp. 1336). Thousand Oaks, CA: Sage.

Qi, H., \& Li, F. (2020). Understanding the study experience of Chinese tourism doctoral students studying overseas. Journal of China Tourism Research, 1-19. doi:https://doi.org/10.1080/19388160.2020.1772929

Ra, Y. A., \& Trusty, J. (2017). Impact of social support and coping on acculturation and acculturative stress of East Asian international students. Journal of Multicultural Counseling and Development, 45(4), 276-291.

Racher, F. (2003). Using conjoint interviews to research the lived experience of elderly rural couples. Nurse Researcher, 19(3), 60-72. 
Racher, F. E., \& Robinson, S. (2003). Are phenomenology and postpositivism strange bedfellows? Western Journal of Nursing Research, 25(5), 464-481.

Radio New Zealand. (2018). International students bring in \$5b each year to New Zealand. Retrieved from https://www.rnz.co.nz/news/national/369911/internationalstudents-bring-in-5b-each-year-to-new-zealand

Ricoeur, P. (1970). Freud and philosophy: An essay on interpretation. Translated by Denis Savage. New Haven: Yale University Press.

Rienties, B., \& Nolan, E.-M. (2014). Understanding friendship and learning networks of international and host students using longitudinal Social Network Analysis. International Journal of Intercultural Relations, 41, 165-180.

Riessman, C. K. (2002). Narrative analysis. In A. M. Huberman \& M. B.Miles (Eds.), The qualitative researcher's companion (pp. 217-270). Thousand Oaks, CA: Sage.

Rinne, T., \& Fairweather, J. (2011). An international comparison of models of cultural and national identity and their implications for New Zealand innovation. Retrieved from http://dspace.lincoln.ac.nz/bitstream/handle/10182/5446/aeru rr 325.pdf?seque $\underline{\text { nce }=1}$

Rizvi, F. (2009). Towards cosmopolitan learning. Discourse: Studies in the Cultural Politics of Education, 30(3), 253-268.

Robertson, M. J., \& Nguyen, M. N. (2020). Robing up: Transactional identity development of Vietnamese PhD students. Asia Pacific Journal of Education, 1-11. doi:https://doi.org/10.1080/02188791.2020.1772197

Robinson, O., Somerville, K., \& Walsworth, S. (2020). Understanding friendship formation between international and host-national students in a Canadian university. Journal of International Intercultural Communication, 13(1), 49-70.

Rogoff, B. (2003). The cultural nature of human development: Oxford university press.

Rogoff, B. (2016). Culture and participation: A paradigm shift. Current Opinion in Psychology, 8, 182-189.

Rogoff, B., \& Angelillo, C. (2002). Investigating the coordinated functioning of multifaceted cultural practices in human development. Human Development, 45(4), 211-225.

Roy, M., Lu, Z., \& Loo, B. (2016). Improving the international student experience: Implications for recruitment and support. Retrieved from www.wes.org/RAS

Ryan, R., Dowler, B., Bruce, S., Gamage, S., \& Morris, A. (2016). The wellbeing of international students in the city of Sydney. Retrieved from Sydney: https://d1yoaun8syyxxt.cloudfront.net/eh183-455357af-5c3a-443a-ab418bad4a48da83-v2

Sadala, M. L. A., \& Adorno, R. d. C. F. (2002). Phenomenology as a method to investigate the experience lived: A perspective from Husserl and Merleau Ponty's thought. Journal of Advanced Nursing, 37(3), 282-293.

Sahu, P. (2020). Closure of universities due to Coronavirus Disease 2019 (COVID-19): Impact on education and mental health of students and academic staff. Cureus, 12(4), 1-6. 
Sam, D. L., \& Berry, J. W. (2010). Acculturation: When individuals and groups of different cultural backgrounds meet. Perspectives on Psychological Science, 5(4), 472-481.

Sanders, P. (1982). Phenomenology: A new way of viewing organizational research. The Academy of Management Review, 7(3), 353-360.

Saravanan, C., Mohamad, M., \& Alias, A. (2019). Coping strategies used by international students who recovered from homesickness and depression in Malaysia. International Journal of Intercultural Relations, 68, 77-87.

Sawir, E., Marginson, S., Deumert, A., Nyland, C., \& Ramia, G. (2008). Loneliness and international students: An Australian study. Journal of Studies in International Education, 12(2), 148-180.

Sawir, E., Marginson, S., Forbes-Mewett, H., Nyland, C., \& Ramia, G. (2012). International student security and English language proficiency. Journal of Studies in International Education, 16(5), 434-454.

Sawir, E., Marginson, S., Nyland, C., Ramia, G., \& Rawlings-Sanaei, F. (2009a). The pastoral care of international students in New Zealand: Is it more than a consumer protection regime? Asia Pacific Journal of Education, 29(1), 45-59.

Sawir, E., Marginson, S., Nyland, C., Ramia, G., \& Rawlings-Sanaei, F. (2009b). The social and economic security of international students: A New Zealand study. Higher Education Policy, 22(4), 461-482. doi:https://doi.org/10.1057/hep.2009.4

Schunk, D. H. (2012). Learning theories: An educational perspective (6th ed.). Boston: Pearson.

Schunk, D. H., Meece, J. R., \& Pintrich, P. R. (2012). Motivation in education: Theory, research, and applications. Boston: Pearson Higher Ed.

Scoop Business. (2020). Rents in New Zealand reach $\$ 515$ for the first time. Retrieved from https://www.scoop.co.nz/stories/BU2002/S00237/rents-in-new-zealand-reach515-for-the-first-time.htm

Selvarajah, C., \& Meyer, D. (2020). Exploring managerial leadership in Vietnam: Where Confucianism meets Dharma. Asia Pacific Business Review, 26(3), 1-27.

Sen, A. (1999). Global justice: Beyond international equity. In I. Kaul, I. Grunberg, \& M. Stern (Eds.), Global Public Goods: International Cooperation in the 21st Century (Vol. 116, pp. 116-125). New York: Oxford University Press.

Sen, A. (2000). Development as freedom. New York: Anchor Books.

Smith, C. (2020). International students and their academic experiences: Student satisfaction, student success challenges, and promising teaching practices. In $U$. Gaulee, S. Sharma, \& K. Bista (Eds.), Rethinking education across borders (pp. 271287). Singapore: Springer.

Smith, J. A. (1994). Reconstructing selves: An analysis of discrepancies between women's contemporaneous and retrospective accounts of the transition to motherhood. British Journal of Psychology, 85(3), 371-392. 
Smith, J. A. (1996). Beyond the divide between cognition and discourse: Using interpretative phenomenological analysis in health psychology. Psychology and Health, 11(2), 261271.

Smith, J. A. (2004). Reflecting on the development of interpretative phenomenological analysis and its contribution to qualitative research in psychology. Qualitative Research in Psychology, 1(1), 39-54.

Smith, J. A. (2015). Qualitative psychology: A practical guide to research methods. Thousand Oaks, CA: Sage.

Smith, J. A. (2017). Interpretative phenomenological analysis: Getting at lived experience. The Journal of Positive Psychology, 12(3), 303-304.

Smith, J. A., Flowers, P., \& Larkin, M. (2009). Interpretative phenomenological analysis: Theory, method and research. Thousand Oaks, CA: Sage.

Smith, J. A., \& Osborn, M. (2007). Qualitative psychology: A practical guide to research methods. In J. A. Smith (Ed.), (pp. 53-80). London: Sage.

Smith, J. A., \& Osborn, M. (2008). Interpretative phenomenological analysis. In J. A. Smith (Ed.), Qualitative psychology: A practical guide to research methods (pp. 53-80). London: Sage.

Smith, R. A., \& Khawaja, N. G. (2011). A review of the acculturation experiences of international students. International Journal of Intercultural Relations, 35(6), 699713. doi:https://doi.org/10.1016/j.ijintrel.2011.08.004

Son, H. N., Chi, D. T. L., \& Kingsbury, A. (2019). Indigenous knowledge and climate change adaptation of ethnic minorities in the mountainous regions of Vietnam: A case study of the Yao people in Bac Kan Province. Agricultural Systems, 176, 102683.

Squires, A. (2009). Methodological challenges in cross-language qualitative research: A research review. International Journal of Nursing Studies, 46(2), 277-287.

Stake, R. E. (1995). The art of case study research. Thousand Oaks, CA: Sage.

Starks, D., \& Nicholas, H. (2017). "Have you noticed any changes upon your return"? Vietnamese alumni after their Australian university experiences. Journal of Intercultural Communication Research, 46(3), 247-272.

Stroebe, M., Van Vliet, T., Hewstone, M., \& Willis, H. (2002). Homesickness among students in two cultures: Antecedents and consequences. British Journal of Psychology, 93(2), 147-168.

Szabó, Á., Papp, Z. Z., \& Luu, L. A. N. (2020). Social contact configurations of international students at school and outside of school: Implications for acculturation orientations and psychological adjustment. International Journal of Intercultural Relations, 77, 69-82.

Taiwan News. (2019). Foreign students in Taiwan 10\% of total university and college students in 2018. Retrieved from https://www.taiwannews.com.tw/en/news/3631340

Tang, C., Gui, X., Chen, Y., \& Magueramane, M. (2018). New to a country: Barriers for international students to access health services and opportunities for design. Paper 
presented at the Proceedings of the 12th EAI International Conference on Pervasive Computing Technologies for Healthcare.

Tay, L., \& Diener, E. (2011). Needs and subjective well-being around the world. Journal of Personality and Social Psychology, 101(2), 354-365.

The United States - Department of Justice. (2020). Country policy and information note Vietnam: Hoa Hao Buddhism. Retrieved from https://www.justice.gov/eoir/page/file/1250811/download

The World Bank. (2008). Vietnam: Higher education and skills for growth. Retrieved from http://documents.worldbank.org/curated/en/439591468338403597/pdf/444280E SWOWHIT1al1July08020081final.pdf

Tho, N. N. (2016). Confucianism and humane education in contemporary Vietnam. International Communication of Chinese Culture, 3(4), 645-671.

Thomas, D. (2020). Factors that contribute to homesickness among students in Thailand. Journal of Social Sciences, 41(1), 136-141.

Thorup-Binger, C., \& Charania, N. A. (2019). Vulnerability and capacities of international students in the face of disasters in Auckland, New Zealand: A qualitative descriptive study. International Journal of Disaster Risk Reduction, 39, 1-8. doi:https://doi.org/10.1016/j.ijdrr.2019.101136

Thurber, C. A., \& Walton, E. A. (2012). Homesickness and adjustment in university students. Journal of American College Health, 60(5), 415-419.

Tian, M., \& Lu, G. (2018). Intercultural learning, adaptation, and personal growth: a longitudinal investigation of international student experiences in China. Frontiers of Education in China, 13(1), 56-92.

Ting-Toomey, S. (2005). Identity negotiation theory: Crossing cultural boundaries. In W. B. Gudykunst (Ed.), Theorizing about intercultural communication (pp. 211-233). Thousand Oaks, CA: Sage.

Todres, L., \& Wheeler, S. (2001). The complementarity of phenomenology, hermeneutics and existentialism as a philosophical perspective for nursing research. International Journal of Nursing Studies, 38(1), 1-8.

Tomkins, L. (2017). Using interpretative phenomenological psychology in organisational research with working carers. In J. Brook \& N. King (Eds.), Applied qualitative research in psychology (pp. 86-100). London: Palgrave.

Tran, A. Q. (2017). Gods, heroes, and ancestors: An interreligious encounter in eighteenthcentury Vietnam. New York: Oxford University Press.

Tran, D. T., Kettle, M., May, L., \& Klenowski, V. (2016). Mediation of institutional English language policies on Vietnamese university teachers' classroom practices. The AsiaPacific Education Researcher, 25(5-6), 791-799.

Tran, L. H. N. (2019). Motivations for studying abroad and immigration intentions: The case of Vietnamese students. Journal of International Students, 9(3), 758-776. 
Tran, L. N. (2011). Holding on to Vietnam: Motivation and the acculturation experience of Vietnamese international students in Australia. (Unpublished doctoral thesis), University of New South Wales, Australia.

Tran, L. T. (2011). Committed, face-value, hybrid or mutual adaptation? The experiences of international students in Australian higher education. Educational Review, 63(1), 7994.

Tran, L. T. (2016). Mobility as 'becoming': A Bourdieuian analysis of the factors shaping international student mobility. British Journal of Sociology of Education, 37(8), 12681289.

Tran, L. T. (2020). How to secure recovery of international student mobility. Retrieved from https://www.universityworldnews.com/post.php?story=20200620071618800

Tran, L. T., \& Gomes, C. (2017). Student mobility, connectedness and identity. In L. T. Tran \& C. Gomes (Eds.), International student connectedness and identity (pp. 1-11). Dordrecht: Springer.

Tran, L. T., \& Marginson, S. (2014). Education for flexibility, practicality and mobility. In L. T. Tran, S. Marginson, H. Do, T. Le, N. Nguyen, T. Vu, T. Pham, \& H. Nguyen (Eds.), Higher education in Vietnam: Flexibility, mobility and practicality in the global knowledge economy. Basingstoke: Palgrave Macmillan.

Tran, L. T., \& Marginson, S. (2018). Internationalisation of Vietnamese higher education: An overview. In L. T. Tran \& S. Marginson (Eds.), Internationalisation in Vietnamese higher education (pp. 1-16). Dordrecht: Springer.

Tran, L. T., Marginson, S., \& Nguyen, N. T. (2014). Internationalisation. In L. Tran, S. Marginson, H. Do, Q. Do, T. Le, N. Nguyen, T. Vu, T. Pham, \& H. Nguyen (Eds.), Higher education in Vietnam - Flexibility, mobility and practicality in the global knowledge economy (pp. 126-149). Basingstoke, Hampshire: Palgrave.

Tran, L. T., Nghia, T. L. H., Nguyen, M., \& Ngo, M. (2019). 'Let go of out-of-date values holding us back': Foreign influences on teaching-learning, research and community engagement in Vietnamese universities. Cambridge Journal of Education, 50(3), 121.

Tran, L. T., Phan, H. L. T., Tan, G., \& Rahimi, M. (2020). 'I changed my strategy and looked for jobs on Gumtree': the ecological circumstances and international graduates' agency and strategies to navigate the Australian labour market. Compare: A Journal of Comparative and International Education, 1-19. doi:https://doi.org/10.1080/03057925.2020.1837613

Tran, L. T., \& Tan, G. (2020). 90,000 foreign graduates are stuck in Australia without financial support: it's a humanitarian and economic crisis in the making. Retrieved from https://theconversation.com/90-000-foreign-graduates-are-stuck-in-australiawithout-financial-support-its-a-humanitarian-and-economic-crisis-in-the-making136717

Tran, L. T., \& Vu, T. T. P. (2018). 'Agency in mobility': towards a conceptualisation of international student agency in transnational mobility. Educational Review, 70(2), 167-187. 
Truong, T. D., Hallinger, P., \& Sanga, K. (2017). Confucian values and school leadership in Vietnam: Exploring the influence of culture on principal decision making. Educational Management Administration \& Leadership, 45(1), 77-100.

Tuffour, I. (2017). A critical overview of interpretative phenomenological analysis: A contemporary qualitative research approach. Journal of Healthcare Communications, 2(4), 1-5.

Tuncay, T., Musabak, I., Gok, D. E., \& Kutlu, M. (2008). The relationship between anxiety, coping strategies and characteristics of patients with diabetes. Health and quality of life outcomes, 6(1), 1-9.

U.S. Embassy and Consulate in Vietnam. (2010). Báo cáo về tự do tôn giáo quốc tế năm 2010. Retrieved from https://vn.usembassy.gov/vi/irfreport2010/

UCAS. (2018). Student accommodation survey 2018/19. Retrieved from https://www.ucasmedia.com/sites/default/files/Student\%20Accommodation\%20S urvey\%202018\%20FINAL WEB.PDF?utm source=web\&utm medium=blog\&utm c ampaign=accsurvey

Ugwu, D. N., \& Adamuti-Trache, M. (2017). Post-graduation plans of international science and engineering doctoral students attending US universities. Journal of International Students, 7(1), 1-21.

UNESCO. (2020). Global Flow of Tertiary-Level Students. Retrieved from http://uis.unesco.org/en/uis-student-flow

United Nations. (1948). Universal declaration of human rights. Retrieved from https://www.un.org/en/universal-declaration-human-rights/

University World News. (2019). From recipient to partner in international education. Retrieved from https://www.universityworldnews.com/post.php?story=20190114124551804

Valle, R. S., King, M., \& Halling, S. (1989). An introduction to existential-phenomenological thought in psychology. In R. S. Valle \& S. Halling (Eds.), Existential-phenomenological perspectives in psychology: Exploring the breadth of human experience (pp. 3-16). New York: Plenum Press.

Vallely, T. J., \& Wilkinson, B. (2008). Vietnamese higher education: Crisis and response. Cambridge, MA: ASH Harvard.

van Manen, M. (1990). Researching lived experience: Human science for an action sensitive pedagogy. Albany, NY: SUNY Press.

Van Manen, M. (2007). Phenomenology of practice. Phenomenology \& Practice, 1, 11-30.

Vergara, M. B., Smith, N., \& Keele, B. (2010). Emotional intelligence, coping responses, and length of stay as correlates of acculturative stress among international university students in Thailand. Procedia - Social and Behavioral Sciences, 5, 1498-1504. doi:https://doi.org/10.1016/j.ijintrel.2011.08.004

Vietnam Ministry of Education and Training. (2020). Hợp tác chiến lược về giáo dục Việt Nam - New Zealand giai đoạn 2020-2023. Retrieved from http://icd.edu.vn/372/hop-tac- 
chien-luoc-ve-giao-duc-viet-nam--new-zealand-giai-doan-20202023.html/BPF/viVN/CMS Cat/Thong-Tin-Hop-Tac-Quoc-Te/CMS Detail/1853

VnExpress. (2016). Du học sinh Việt Nam đang học tập tại những nước nào? Retrieved from https://vnexpress.net/infographics/du-hoc-sinh-viet-nam-dang-hoc-tap-tai-nhungnuoc-nao-3493240.html

Vu, H. T. (2013). Vietnamese international students' experiences in a New Zealand university: A narrative study. (Unpublished master's thesis), Victoria University of Wellington, Wellington, New Zealand.

Vu, H. T., \& Doyle, S. (2014). Across borders and across cultures: Vietnamese students' positioning of teachers in a university twinning programme. Journal of Education for Teaching, 40(3), 267-283.

Vuong, Q.-H., Bui, Q.-K., La, V.-P., Vuong, T.-T., Nguyen, V.-H. T., Ho, M.-T., . . . Ho, M.-T. (2018). Cultural additivity: behavioural insights from the interaction of Confucianism, Buddhism and Taoism in folktales. Palgrave Communications, 4(1), 1-15.

Wagstaff, C., Jeong, H., Nolan, M., Wilson, T., Tweedlie, J., Phillips, E., ... Holland, F. (2014). The accordion and the deep bowl of spaghetti: Eight researchers' experiences of using IPA as a methodology. The Qualitative Report, 19(24), 1-15.

Wang, C., Whitehead, L., \& Bayes, S. J. (2017). "They are friendly but they don't want to be friends with you": A narrative inquiry into Chinese nursing students' learning experiences in Australia. Journal of Nursing Education and Practice, 7(8), 27-36.

Wang, X. (2015). An interpretive phenomenological analysis of Chinese students' satisfaction with postgraduate university study and life in New Zealand. (Unpublished master's thesis), Victoria University of Wellington, New Zealand.

Ward, C. (1997). Culture learning, acculturative stress, and psychopathology: Three perspectives on acculturation. Applied Psychology, 46(1), 58-62.

Ward, C. (2008). Thinking outside the Berry boxes: New perspectives on identity, acculturation and intercultural relations. International Journal of Intercultural Relations, 32(2), 105-114. doi:https://doi.org/10.1016/j.ijintrel.2007.11.002

Ward, C., Bochner, S., \& Furnham, A. (2001). The psychology of culture shock. London: Routledge.

Ward, C., \& Geeraert, N. (2016). Advancing acculturation theory and research: The acculturation process in its ecological context. Current Opinion in Psychology, 8, 98104.

Ward, C., \& Kus, L. (2012). Back to and beyond Berry's basics: The conceptualization, operationalization and classification of acculturation. International Journal of Intercultural Relations, 36(4), 472-485.

Watkins, D. A., \& Biggs, J. B. (2001). Teaching the Chinese learner: Psychological and pedagogical perspectives. Hong Kong: CERC and ACER.

Wearing, A., Le, H., Wilson, R., \& Arambewela, R. (2015). The international student's experience: An exploratory study of students from Vietnam. The International Education Journal: Comparative Perspectives, 14(1), 71-89. 
Weiss, R. S. (1973). Loneliness: The experience of emotional and social isolation. Cambridge: MIT Press.

Wilkins, S., Balakrishnan, M. S., \& Huisman, J. (2012). Student choice in higher education: Motivations for choosing to study at an international branch campus. Journal of Studies in International Education, 16(5), 413-433.

Willig, C. (2013). Introducing qualitative research in psychology. UK: McGraw-Hill Education.

Willig, C., \& Stainton-Rogers, W. (2017). The Sage handbook of qualitative research in psychology. London, UK: Sage.

World Education News and Reviews. (2017). Education in Vietnam. Retrieved from https://wenr.wes.org/2017/11/education-in-vietnam

Wu, Q. (2015). Re-examining the "Chinese learner": a case study of mainland Chinese students' learning experiences at British Universities. Higher Education, 70(4), 753766.

Xing, X., Popp, M., \& Price, H. (2020). Acculturation strategies of Chinese university students in the United States. Journal of Advances in Education Research, 5(1), 11-24.

Xu, Y. Z. (2002). A thesis on the spreading and influence of Taoism in Vietnam. Journal of Historical Science, 7, 015.

Yan, K. (2017). Chinese international students' stressors and coping strategies in the United States. Singapore: Springer.

Yan, Z. (2020). Acculturation and well-being among international students: Challenges and opportunities. In U. Gaulee, S. Sharma, \& K. Bista (Eds.), Rethinking Education Across Borders (pp. 303-315). Singapore: Springer.

Yates, L., \& Trang, N. T. Q. (2012). Beyond a discourse of deficit: The meaning of silence in the international classroom. International Education Journal: Comparative Perspectives, 11(1), 22-34.

Ye, L., \& Edwards, V. (2015). Chinese overseas doctoral student narratives of intercultural adaptation. Journal of Research in International Education, 14(3), 228-241.

Yoon, E., Langrehr, K., \& Ong, L. Z. (2011). Content analysis of acculturation research in counseling and counseling psychology: A 22-year review. Journal of Counseling Psychology, 58(1), 83-96.

Young, J. T. (2017). Confucianism and accents: Understanding the plight of the Asian international student in the US. Journal of International Students, 7(3), 433-448.

Yu, B., Bodycott, P., \& Mak, A. S. (2019). Language and interpersonal resource predictors of psychological and sociocultural adaptation: International students in Hong Kong. Journal of Studies in International Education, 23(5), 572-588.

Yu, Q., Foroudi, P., \& Gupta, S. (2019). Far apart yet close by: Social media and acculturation among international students in the UK. Technological Forecasting and Social Change, 145, 493-502. 
Yu, Y., \& Moskal, M. (2019). Missing intercultural engagements in the university experiences of Chinese international students in the UK. Compare: A Journal of Comparative and International Education, 49(4), 654-671.

Zhang, J., \& Goodson, P. (2011). Predictors of international students' psychosocial adjustment to life in the United States: A systematic review. International Journal of Intercultural Relations, 35(2), 139-162. doi:https://doi.org/10.1016/j.ijintrel.2010.11.011

Zhang, X. (2018). Chinese international students in New Zealand: Views of citizenship and democracy. Citizenship Teaching \& Learning, 13(2), 241-252.

Zhou, Y., Jindal-Snape, D., Topping, K., \& Todman, J. (2008). Theoretical models of culture shock and adaptation in international students in higher education. Studies in Higher Education, 33(1), 63-75.

Zhu, J. (2016). Chinese overseas students and intercultural learning environments: Academic adjustment, adaptation and experience. London: Macmillan.

Ziguras, C., \& McBurnie, G. (2011). International student mobility in the Asia-Pacific: From globalization to regional integration? In S. Marginson, S. Kaur, \& E. Sawir (Eds.), Higher education in the Asia-Pacific: Strategic responses to globalization (pp. 123140). Dordrecht, Netherlands: Springer.

Ziguras, C., \& Tran, L. (2020). The coronavirus outbreak is the biggest crisis ever to hit international education. Retrieved from https://theconversation.com/thecoronavirus-outbreak-is-the-biggest-crisis-ever-to-hit-international-education$\underline{131138}$ 


\section{Appendices}

\section{Appendix A | Human Ethics Approval Letter}

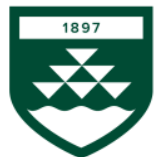

Victopla univessiry or WELLINGTON TE HERENGA WAKA
Phone

Email

\begin{tabular}{|c|c|}
\hline TO & Hau Ho \\
\hline FROM & Associate Professor Judith Loveridge, Convenor, Human Ethics Committee \\
\hline DATE & 20 October 2017 \\
\hline PAGES & 1 \\
\hline SUBJECT & $\begin{array}{l}\text { Ethics Approval Number: } 25280 \\
\text { Title: An interpretative phenomenological analysis of Vietnamese } \\
\text { postgraduate international students' experiences in New Zealand }\end{array}$ \\
\hline
\end{tabular}

Thank you for your application for ethical approval, which has now been considered by the Human Ethics Committee.

Your application has been approved from the above date and this approval is valid for three years. If your data collection is not completed by this date you should apply to the Human Ethics Committee for an extension to this approval.

Best wishes with the research.

Kind regards,

Judith Loveridge

Convenor, Victoria University of Wellington Human Ethics Committee 


\section{Appendix B | Participant Information Sheet}

\section{English version}

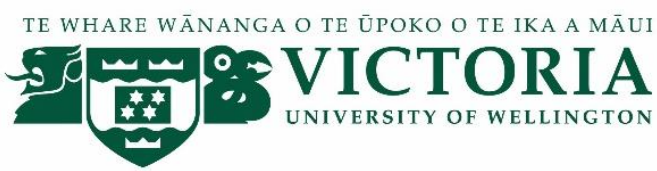

\section{An Interpretative Phenomenological Analysis of Vietnamese International Postgraduate Students' Experiences in New Zealand}

\section{INFORMATION SHEET FOR INTERVIEW PARTICIPANTS}

You are invited to take part in this research. Please read this information before deciding whether or not to take part. If you decide to participate, thank you. If you decide not to participate, thank you for considering this request.

\section{Who am I?}

My name is Ho Trung Hau and I am a doctoral student in Faculty of Education at Victoria University of Wellington. This research project is work towards my thesis.

\section{What is the aim of the project?}

The aim of this study is to explore the lived experiences of Vietnamese Master's students in New Zealand. Specifically, I aim to examine students' transformative experiences and challenges in academic, social, and personal lives, coping strategies, and the relation between their motivations to study overseas and their experiences.

This research has been approved by the Victoria University of Wellington Human Ethics Committee (reference number: 25280).

\section{How can you help?}

If you agree to participate and you are selected, I will interview you face-to-face three times. The interviews will be conducted in Vietnamese. The first interview will mainly involve getting your background information (e.g., what you were doing before coming to New 
Zealand?). In the second interview, I will ask you questions about different aspects of your experiences (e.g., course experiences, coping strategies). The third interview aims to clarify certain points or to gain more detailed information about your responses in the second interview. Each interview will take approximately 60 minutes. I will audio record the interview with your permission and write it up later. You can choose to not answer any question or stop the interview at any time, without giving a reason. You can withdraw from the study by contacting me at any time before the data collection is finished (30 July 2018). If you withdraw, the information you provided will be destroyed.

\section{What will happen to the information you give?}

This research is confidential. This means that the researchers named below will be aware of your identity, but the research data will be combined, and your identity will not be revealed in any reports, presentations, or public documentation. Only my supervisors and I will read the notes or transcript of the interview. The interview transcripts, summaries and any recordings will be kept securely and destroyed one year after the completion of the thesis.

\section{What will the project produce?}

The information from my research will be used in my $\mathrm{PhD}$ thesis and for publication in academic journals, book chapters, or presentations at conferences.

\section{If you accept this invitation, what are your rights as a research participant?}

You do not have to accept this invitation if you don't want to. If you do decide to participate, you have the right to:

- choose not to answer any question;

- ask for the recorder to be turned off at any time during the interview;

- withdraw from the study before the data collection and analysis are finished, and inform the researcher via email;

- ask any questions about the study at any time;

- receive a copy of the Vietnamese transcript of your interview recording and its English translation; 
- read over and comment on a written summary of your interview;

- agree on another name for me to use rather than your real name;

- be able to read a summary report of findings from this research by emailing the researcher to request a copy;

- withdraw from the study by contacting me at any time before the data collection is finished (30 July 2018).

\section{If you have any questions or problems, who can you contact?}

If you have any questions, either now or in the future, please feel free to contact either:

PhD Student:

Name: Ho Trung Hau

Phone:

Email:

\section{Human Ethics Committee information}

If you have any concerns about the ethical conduct of the research, you may contact the

or telephone:

\section{Supervisors:}

Dr Stephanie Doyle

Phone:

Email:

Associate Professor Joanna Kidman

Phone:

Email:

\section{Victoria University HEC Convenor: Associate Professor Susan Corbett. Email:}




\section{Vietnamese Version}

TE WHARE WĀNANGA O TE ŪPOKO O TE IKA A MĀUI

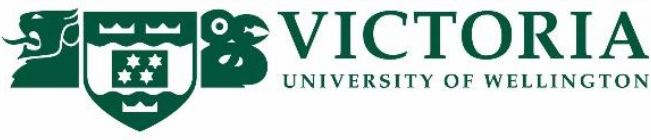

\section{Phân Tích Hiện Tượng Diễn Giải Trải Nghiệm Của Sinh Viên Quốc Tế Từ Việt Nam Bậc Sau Đại Học Ở New Zealand BẢN THÔNG TIN CHO NGƯờI THAM GIA PHỎNG VẤN}

Bạn được mời tham gia vào nghiên cứu này. Các bạn vui lòng đọc thông tin này trước khi quyết định xem có tham gia hay không. Nếu bạn quyết định tham gia, xin cảm ơn các bạn. Nếu các bạn quyết định không tham gia, tôi cảm ơn các bạn đã xem qua lời mời của tôi.

\section{Tôi là ai?}

Tôi tên Hồ Trung Hậu, nghiên cứu sinh Khoa Giáo dục của trường Đại học Victoria Wellington. Đây là nghiên cứu được sử dụng cho luận văn của tôi.

\section{Mục đích nghiên cứu là gì?}

Mục đích của nghiên cứu này là để tìm hiểu trải nghiệm sống của sinh viên Việt Nam bậc Thạc sĩ ở New Zealand. Cụ thể là, tôi sẽ xem xét sự thay đổi của bản thân các bạn, các khó khăn liên quan đến học tập, văn hóa xã hội, cách thức giải quyết khó khăn, sự liên hệ giữa động lực du học và các trải nghiệm.

Nghiên cứu này đã được chấp thuận bởi Hội đồng Đạo đức trong nghiên cứu con người của trường Đại học Victoria-Wellington [số tham chiếu: 25280].

\section{Bạn có thể giúp như thế nào?}

Nếu bạn đồng ý tham gia và được lựa chọn, tôi sẽ phỏng vấn trực tiếp các bạn ba lần. Phỏng vấn sẽ được tiến hành bằng tiếng Việt. Buổi phỏng vấn thứ nhất nhằm thu thập một số thông tin cá nhân của các bạn (ví dụ, bạn làm nghề gì trước khi đến New Zealand?). Trong buổi phỏng vấn thứ hai, tôi sẽ hỏi các bạn về các khía cạnh trải nghiệm như đã đề cập ở phần trước (ví dụ, trải nghiệm học tập, cách giải quyết khó khăn gặp phải). Buổi phỏng vấn thứ ba 
nhằm làm rõ một số thông tin hoặc tìm hiểu chi tiết về câu trả lời của các bạn ở buổi phỏng vấn thứ hai. Mỗi buổi phỏng vấn sẽ kéo dài khoảng 60 phút. Tôi sẽ thu âm buổi phỏng vấn với sự đồng ý của bạn và ghi chép lại sau. Bạn có thể chọn không trả lời bất kỳ câu hỏi nào hoặc dừng buổi phỏng vấn bất kỳ lúc nào mà không phải nêu lý do. Bạn có quyền rút khỏi nghiên cứu bất kỳ lúc nào bằng cách liên lạc với tôi trước khi việc thu thập dữ liệu hoàn thành (30/7/2018). Nếu rút khỏi nghiên cứu, thông tin của các bạn sẽ được hủy bỏ.

\section{Thông tin các bạn cung cấp sẽ được xử lý thế nào?}

Nghiên cứu này mang tính bí mật. Điều này có nghĩa là những người nghiên cứu kể tên bên dưới sẽ biết thông tin nhận dạng của bạn nhưng dữ liệu nghiên cứu sẽ được tổng hợp và nhận dạng của các bạn sẽ không được tiết lộ trong bất kỳ báo cáo, trình bày, hay tài liệu được công bố nào. Chỉ có tôi và các giáo viên hướng dẫn của tôi đọc được các ghi chú hoặc bản ghi lại của các buổi phỏng vấn. Các bản ghi lại, tóm tắt, và thu âm sẽ được giữ an toàn và tiêu hủy sau khi hoàn thành luận văn một năm.

\section{Nghiên cứu này sẽ tạo ra sản phẩm gì?}

Thông tin từ nghiên cứu của tôi được dùng cho luận văn tiến sĩ của tôi và cho việc xuất bản trong các tạp chí khoa học, sách, hoặc trình bày ở các hội nghị.

\section{Nếu bạn chấp nhận lời mời, quyền của bạn là gì?}

Bạn không cần phải chấp nhận lời mời này nếu bạn không muốn. Nếu bạn quyết định tham gia, bạn có quyền:

- chọn không trả lời bất kỳ câu hỏi nào;

- đề nghị ngừng ghi âm bất kỳ lúc nào trong buổi phỏng vấn;

- rút khỏi nghiên cứu trước khi việc thu thập và phân tích dữ liệu kết thúc, và thông báo cho người nghiên cứu biết thông qua email;

- hỏi bất kỳ câu hỏi nào về nghiên cứu bất kỳ lúc nào;

- nhận bản ghi lại bằng tiếng Việt và bản dịch tiếng Anh của thu âm buổi phỏng vấn;

- đọc lại và nhận xét bản tóm tắt buổi phỏng vấn;

- đồng ý sử dụng một tên gọi khác thay cho tên thật; 
- có thể đọc bản báo cáo tóm tắt những phát hiện của nghiên cứu này bằng cách gởi email yêu cầu cho người thực hiện nghiên cứu;

- rút khỏi nghiên cứu bằng cách liên lạc với tôi bất kỳ lúc nào trước trước khi quá trình thu thập dữ liệu kết thúc (30/7/2018).

\section{Nếu bạn có thắc mắc, bạn có thể liên lạc với ai?}

Nếu bạn có bất kỳ thắc mắc nào, bây giờ hoặc sau này, vui lòng liên hệ một trong các cá nhân sau:

Nghiên cứu sinh:

Hồ Trung Hậu

Đt:

Email:
Giáo viên hướng dẫn:

TS. Stephanie Doyle

$Đ t:$

Email:

PGS. Joanna Kidman

$Đ t:$

Email:

\section{Thông tin về Hội đồng đạo đức trong nghiên cứu con người}

Nếu bạn bận tâm về các vấn đề đạo đức của nghiên cứu, bạn có thể liên hệ Chủ tịch Hội đồng đạo đức trong nghiên cứu con người của Đại học Victoria - Wellington: PGS. Susan Corbett. Email: hoặc đt: 


\section{Appendix C | Participant Consent Form}

\section{English Version}

TE WHARE WĀNANGA O TE ŪPOKO O TE IKA A MĀUI

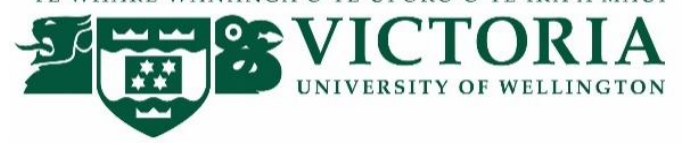

\section{An Interpretative Phenomenological Analysis of Vietnamese International Postgraduate Students' Experiences in New Zealand}

\section{CONSENT FOR INTERVIEW PARTICIPANTS}

This consent form will be held for five years.

Researcher: Ho Trung Hau, Faculty of Education, Victoria University of Wellington.

- I have read the Information Sheet and the project has been explained to me. My questions have been answered to my satisfaction. I understand that I can ask further questions at any time.

- I agree to take part in an audio recorded interview.

I understand that:

- I may withdraw from this study at any point before the data collection and analysis are finished (30 July 2018), and any information that I have provided will be returned to me or destroyed.

- The identifiable information I have provided will be destroyed one year after the completion of the thesis.

- Any information I provide will be kept confidential to the researcher and the supervisors.

- I understand that the results will be used for a PhD thesis, academic publications or presented to conferences.

- My name will not be used in reports, nor will any information that would identify me.

- I would like a copy of the transcript of my interview.

Yes $\square$

No $\square$

- I would like a summary of my interview.

Yes

Yes

No $\square$

- I would like to receive a copy of the final report and have added my email address below.

No $\square$

Signature of participant:

Name of participant:

Date:

Email: 


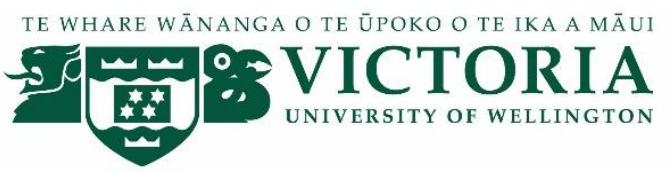

\section{Phân Tích Hiện Tượng Diễn Giải Trải Nghiệm Của Sinh Viên Quốc Tế Từ Việt Nam Bậc Sau Đại Học Ở New Zealand}

\section{ĐơN ĐỒNG Ý THAM GIA PHỎNG VẤN CỦA SINH VIÊN}

Đơn này sẽ được lưu trong năm năm

Người nghiên cứu: Hồ Trung Hậu, Khoa Giáo dục, Đại học Victoria - Wellington

- Tôi đã đọc bản thông tin nghiên cứu và đã được giải thích về dự án này. Các thắc mắc của tôi đã được giải đáp thỏa đáng. Tôi hiểu rằng tôi có thể nêu thêm thắc mắc bất kỳ lúc nào.

- Tôi đồng ý tham gia vào buổi phỏng vấn được ghi âm này.

Tôi hiểu rằng:

- Tôi có thể rút khỏi nghiên cứu này bất kỳ lúc nào trước khi việc thu thập và phân tích dữ liệu kết thúc (30/7/2018), và bất kỳ thông tin nào tôi cung cấp sẽ được trả lại cho tôi hoặc tiêu hủy.

- Những thông tin có thể nhận diện tôi đã cung cấp sẽ được tiêu hủy một năm sau khi hoàn thành luận văn.

- Thông tin tôi cung cấp sẽ được bảo mật bởi người nghiên cứu và người hướng dẫn.

- Tôi hiểu rằng các kết quả sẽ được sử dụng cho luận văn tiến sĩ, các ấn bản học thuật hoặc được trình bày ở các hội thảo.

- Tên tôi cũng như bất kỳ thông tin nào có thể nhận diện tôi sẽ không được dùng trong các báo cáo.

- Tôi muốn nhận một bản ghi lại các buổi phỏng vấn của tôi. Có $\square$ Không $\square$

- Tôi muốn nhận bản tóm tắt các buổi phỏng vấn của tôi. $\quad$ Có $\square$ Không $\square$

- Tôi muốn nhận bản báo cáo hoàn tất theo email dưới đây. Có $\square$ Không $\square$

Chữ ký của người tham gia:

Tên của người tham gia:

Ngày:

Email: 


\section{Appendix D | Participant Recruitment Flier}

\section{English version}

\section{Are you a Vietnamese master's student?}

I am currently recruiting participants for a study on Vietnamese students' experiences in New Zealand. This study will contribute to understanding of the experiences of Vietnamese students in this country.

\section{Who can participate?}

Vietnamese master's students

\section{What will I be required to do?}

Participate in three interviews in Vietnamese.

\section{Where will the interviews take place?}

Anywhere that is convenient for you.

\section{Thank you gift.}

\$20 voucher for each interview.

Interested? Contact me at:

(Ho Trung Hau)

\section{Please share!}

This study is conducted under the supervision of Dr Stephanie Doyle and Associate Professor Joanna Kidman in the School of Education, Victoria University of Wellington. 


\section{Vietnamese version}

\section{Bạn là sinh viên cao học Việt Nam?}

Tôi hiện đang tìm kiếm đối tượng cho một nghiên cứu về trải nghiệm của sinh viên Việt Nam ở New Zealand.

Nghiên cứu này góp phần tìm hiểu rõ hơn về trải nghiệm của sinh viên Việt Nam ở quốc gia này.

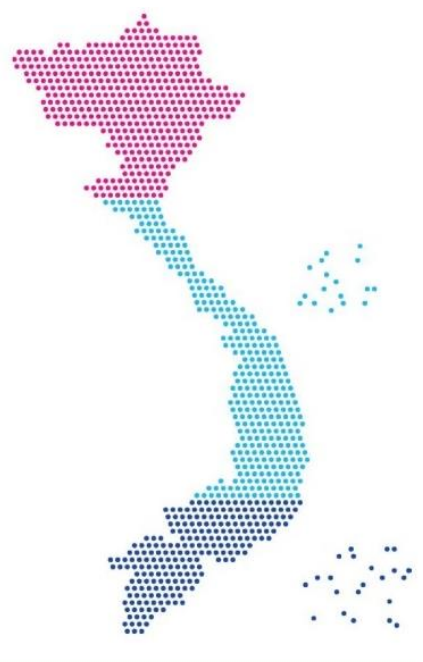

\section{Ai có thể tham gia?}

Sinh viên Việt Nam đang học Thạc sĩ tại Đại học Victoria-Wellington (VUW).

\section{Bạn sẽ được yêu cầu làm gì?}

Tham gia ba buổi phỏng vấn bằng tiếng Việt.

\section{Phỏng vấn được tiến hành ở đâu? \\ Ở nơi nào tiện lợi cho bạn.}

\section{Quà cảm ơn.}

Phiếu mua hàng \$20/buổi phỏng vấn.

Nếu bạn quan tâm, hãy liên lạc với tôi:

(Hồ Trung Hậu)

Hãy chia sẻ thông báo này.

Xin cảm ơn!

Nghiên cứu này được thực hiện dưới sự hướng dẫn của TS. Stephanie Doyle và PGS. Joanna Kidman thuộc Khoa Giáo dục, Đại học Victoria - Wellington. 


\section{Appendix E | Indicative Interview Questions}

\section{Prompts (in English)}

To gain further information:

- How/Where/Why or When did that happen?

- Could you give me some examples?

- What was useful to you during that difficult time?

- What did you enjoy/value?

- What is confusing/challenging to you?

- What made a difference?

- What did you not expect?

\section{Prompts (in Vietnamese)}

Nhằm lấy thêm thông tin:

- Điều đó đã xảy ra như thế nào/ở đâu/tại sao/khi nào?

- Bạn cho ví dụ được không?

- Điều gì giúp bạn vượt qua khó khăn?

- Điều gì bạn trân trọng?

- Điều gì gây khó khăn cho bạn?

- Điều gì tạo nên sự khác biệt?

- Điều gì bạn không ngờ?
To clarify details:

- What did you mean by that?

- Did you mean...?
Nhằm làm rõ thông tin:

- Bạn muốn nói gì?

- Có phải ý bạn là...?

To elicit the participants' reflection, Nhằm nắm được cảm xúc và suy nghĩ của emotions or feelings:

đối tượng nghiên cứu:

- How did you feel when...?

- Bạn cảm thấy như thế nào khi...?

- How did that affect you?

- What were your thoughts at that time?

- Điều đó ảnh hưởng bạn như thế nào?

- What did that mean to you?

- Bạn suy nghĩ gì lúc đó?

- Điều đó có ý nghĩa gì? 


\section{First interview}

\section{English version}

- Could you please tell me about your background (family, job, and education)?

- Could you tell me about your personalities?

- Have you lived away from home? How do you think this might relate to your living experiences in New Zealand?

- Do you usually talk to your family?

- What motivated you to study overseas? For choosing New Zealand? For enrolling in Victoria University? For getting the qualification?

- Can you tell me about your previous study? How do your prior study experiences relate to your decision to study overseas?

\section{Vietnamese version}

- Bạn có thể cho biết vài thông tin cá nhân của bạn (gia đình, công việc, giáo dục)?

- Bạn tự nhận xét về tính cách bản thân mình như thế nào?

- Bạn có từng sống xa gia đình chưa? Việc đó có ảnh hưởng như thế nào đối với cuộc sống của bạn lúc này?

- Bạn thường liên lạc với gia đình không?

- Tại sao bạn muốn đi du học? Tại sao bạn chọn New Zealand, trường Đại học Victoria-Wellington, và ngành học này?

- Bạn có thể nói về việc học đại học trước đây của bạn không? Những trải nghiệm đó có liên quan như thế nào đến việc bạn quyết định du học?

- What would you like to do after • Bạn dự định làm gì sau khi tốt nghiệp? graduation?

- How did you prepare for study and life in New Zealand (e.g., visa application, accommodation, and funding support)? Your source of information and support?

- Did you come to New Zealand by yourself or together with someone? How was your journey?

- Bạn đã chuẩn bị cho học tập và cuộc sống ở New Zealand như thế nào (xin visa, tìm nơi ở, và tài chính)? Bạn tìm kiếm thông tin và hỗ trợ từ đâu?

- Bạn đến New Zealand một mình hay đi cùng bạn bè? Chuyến đi của bạn như thế nào?

- Are you working part-time?

- Bạn có đi làm thêm không?

- How did you plan to pay for your tuition fees and living costs? Any difficulties?

- Bạn có thể chia sẻ về kế hoạch tài chính cho du học như thế nào? Có khó khăn gì không?

- Has your financial situation changed since you came here? What has led to the change?

- Kế hoạch của bạn có thay đổi từ lúc bạn đến đây không? Điều gì dẫn đến sự thay đổi?

- Is there anything else that we did not discuss and that you wish to share with me or to comment?
- Có điều gì chúng ta chưa thảo luận mà bạn lại muốn nói thêm hoặc bình luận thêm không? 


\section{Second interview}

\section{English version}

- Do you want to clarify or add any details to what we discussed in our earlier interview before we get started?

\section{Academic experiences}

- What did you do in your first days at the university? Your early impressions about university life? Your first week? Your first assignment?

- Tell me about difficulties with the new learning environment? Do you seek help from support services? How have things changed with time?

- What do you feel about your academic progress?

- What are your overall impressions of your educational experiences so far?

\section{Socio-cultural and personal experiences}

- Tell me about your networks. What do you think about making friends with local students/other international students?

- Please tell me about your initial impressions of daily life here. What did you do on your first days?

- Tell me about your accommodation.

- Tell me about your flatmates. What are your experiences with them?

- Have you taken part in any extracurricular activities?

- What are your overall impressions of your daily experiences so far?

\section{Vietnamese version}

- Trước khi mình tiến hành buổi phỏng vấn, bạn có muốn bổ sung thêm gì vào nội dung đã nói trong buổi trước không?

\section{Trải nghiệm học tập}

- Bạn làm gì trong những ngày đầu tiên đến trường? Ấn tượng đầu tiên về môi trường học tập ở đây như thế nào? Tuần học đầu tiên? Bài tập đầu tiên?

- Điều gì gây khó khăn cho bạn? Bạn có gặp khó khăn gì không? Bạn có dùng dịch vụ của nhà trường không? Mọi việc thay đổi như thế nào?

Bạn cảm thấy như thế nào về tiến độ học tập của mình?

- Trải nghiệm chung là gì?

\section{Trải nghiệm văn hóa xã hội}

- Hãy kể về những mối quan hệ của bạn. Bạn nghĩ gì về việc kết bạn với sinh viên New Zealand/các sinh viên quốc tế khác?

- Hãy nói về những ấn tượng ban đầu của bạn về cuộc sống ở đây. Những ngày đầu tiên của bạn như thế nào?

- Hãy nói về chỗ ở của bạn.

- Hãy kể về người ở chung với bạn. Tương tác của bạn với họ như thế nào?

- Bạn có tham gia vào hoạt động ngoại khóa nào không?

Ấn tượng chung của bạn là gì? 
- [For participants with partners/children left behind] Can you tell me a little about your family? What are the advantages/disadvantages? How have you dealt with challenges?

- How have things changed with time?

- Is there anything else that we did not discuss and that you wish to share with me or to comment?
- [Dành cho người tham gia phỏng vấn có gia điinh] Bạn có thể cho tôi biết chút ít về gia đình của bạn không? Có khó khăn gì không? Bạn đã giải quyết các khó khăn như thế nào?

- Mọi thứ đã thay đổi theo thời gian như thế nào?

- Có điều gì chúng ta chưa thảo luận mà bạn lại muốn nói thêm không? 


\section{Third interview}

\section{English version}

- Do you want to clarify or add any details to what we discussed in our earlier interview before we get started?

- Please describe your experiences since our last interview. How have you been feeling over the last few months? Have there been any changes since our last interview in terms of your study and your life?

- How do you feel about your academic progress now?

- Please tell me about how you have changed after being in New Zealand for nearly one year (e.g., language skills, friendship network, communication skills, level of confidence and personal views).

- Please tell me about your plans after graduation.

- What suggestions would you like to give to prospective international students? To the university?

- Is there anything else that we did not discuss and that you wish to share with me or to comment?

- We have finished the three interviews, but please feel free to contact me over the next few months if you wish to add or clarify something.

- Can I contact you later if I have some follow-up questions?

\section{Vietnamese version}

- Trước khi mình tiến hành buổi phỏng vấn, bạn có muốn bổ sung thêm gì vào nội dung đã nói trong buổi trước không?

- Vui lòng nói về trải nghiệm của bạn kể từ buổi phỏng vấn vừa qua của chúng ta. Có thay đổi gì kể từ cuộc phỏng vấn vừa qua của chúng ta liên quan đến việc học của bạn và cuộc sống của bạn?

- Bạn cảm thấy thế nào về tiến độ học tập của bạn đến giờ?

- Hãy nói cho tôi biết bạn đã thay đổi như thế nào trong một năm qua (kỹ năng ngôn ngữ, mạng lưới bạn bè, kỹ năng giao tiếp, mức độ tự tin, và quan điểm sống)?

- Kế hoạch của bạn sau khi tốt nghiệp là gì?

- Có lời khuyên nào bạn muốn cung cấp cho sinh viên quốc tế tương lai? Góp ý cho trường đại học?

- Bạn có muốn bổ sung hoặc bình luận thêm điều gì không?

- Chúng ta đã hoàn thành ba buổi phỏng vấn, nhưng bạn có thể liên lạc để cung cấp thêm thông tin nếu bạn muốn.

- Tôi có thể liên lạc lại bạn để hỏi thêm một số câu hỏi nếu có không? 


\section{Appendix F | Summary of Studies on Vietnamese International Students' Experiences}

\begin{tabular}{|c|c|c|c|c|}
\hline Author/Year & Country & $\begin{array}{l}\text { Participants/Sample } \\
\text { size }\end{array}$ & Purposes and Methodology & Key findings \\
\hline \multirow[t]{2}{*}{ Phan (2001) } & Australia & 4 PG students & $\begin{array}{l}\text { - Exploring how the notion of } \\
\text { 'politeness' influences the way } \\
\text { students write academic English } \\
\text { - Interviews/Thematic analysis }\end{array}$ & $\begin{array}{l}\text { - There were differences between } \\
\text { Vietnamese and English essays in terms of } \\
\text { expression of critical analytical thinking and } \\
\text { referencing conventions. English writing was } \\
\text { more critical than Vietnamese writing. } \\
\text { English writing had referencing conventions } \\
\text { while Vietnamese writing did not usually } \\
\text { require referencing. }\end{array}$ \\
\hline & & & & $\begin{array}{l}\text { - The participants knew how to write good } \\
\text { English essays and did not encounter serious } \\
\text { problems with English writing. }\end{array}$ \\
\hline \multirow[t]{3}{*}{ Dao $(2005) *$} & NZ & 48 UG and PG students & $\begin{array}{l}\text { - Investigating the library use of } \\
\text { students at one university }\end{array}$ & \multirow{2}{*}{$\begin{array}{l}\text { - The students visited the library to study and } \\
\text { use the Internet. They liked using the Internet } \\
\text { to search for information and preferred print } \\
\text { documents. The library catalogue was } \\
\text { important. }\end{array}$} \\
\hline & & & $\begin{array}{l}\text { - Questionnaire/Descriptive } \\
\text { statistics }\end{array}$ & \\
\hline & & & & $\begin{array}{l}\text { - They were satisfied with library services and } \\
\text { information sources. }\end{array}$ \\
\hline Do (2007) & USA & 8 UG students & $\begin{array}{l}\text { - Exploring students' adaptation } \\
\text { experiences }\end{array}$ & $\begin{array}{l}\text { - The students relied on themselves rather } \\
\text { than support services for personal problems. }\end{array}$ \\
\hline
\end{tabular}




\begin{tabular}{|c|c|c|c|c|}
\hline Author/Year & Country & $\begin{array}{l}\text { Participants/Sample } \\
\text { size }\end{array}$ & Purposes and Methodology & Key findings \\
\hline & & & $\begin{array}{l}\text { - Qualitative case study } \\
\text { - Weekly diaries over one } \\
\text { semester, interviews } \\
\text { - Data analysed using Stake's } \\
\text { (1995) approach }\end{array}$ & $\begin{array}{l}\text { - Although facing language-related issues in } \\
\text { the classroom, they had good academic } \\
\text { performance. }\end{array}$ \\
\hline Brisset et al. (2010) & France & $\begin{array}{l}112 \text { Vietnamese UG } \\
\text { and PG students } \\
101 \text { French UG and PG } \\
\text { students }\end{array}$ & $\begin{array}{l}\text { - Exploring factors affecting } \\
\text { Vietnamese students' adaptation } \\
\text { to France } \\
\text { - Quantitative/Correlational, } \\
\text { Exploratory and path analyses }\end{array}$ & $\begin{array}{l}\text { - Attachment intimacy and trait-anxiety } \\
\text { moderated by psychological distress were } \\
\text { relevant to adaptation for both Vietnamese } \\
\text { and French students. } \\
\text { - Co-national identification was relevant to } \\
\text { the Vietnamese students'adaptation process. } \\
\text { - Attachment issues appeared to have a } \\
\text { greater impact on adaptation for the } \\
\text { Vietnamese than the French students. }\end{array}$ \\
\hline L. N. Tran (2011) * & Australia & $\begin{array}{l}51 \text { UG and graduate } \\
\text { students participating } \\
\text { in interviews } \\
301 \text { UG and graduate } \\
\text { students responding } \\
\text { to survey }\end{array}$ & $\begin{array}{l}\text { - Students' motivations to study } \\
\text { overseas and acculturation } \\
\text { experiences } \\
\text { - Mixed-methods triangulation } \\
\text { design-convergence model } \\
\text { - Interviews/correlational } \\
\text { analysis and survey/grounded } \\
\text { theory approach }\end{array}$ & $\begin{array}{l}\text { - Students' motivation included self- } \\
\text { development, education, stimulation, and } \\
\text { wealth. } \\
\text { - Challenges included language difficulties, } \\
\text { interactions with local students, } \\
\text { loneliness/isolation, homesickness, and } \\
\text { academic adjustment. }\end{array}$ \\
\hline
\end{tabular}




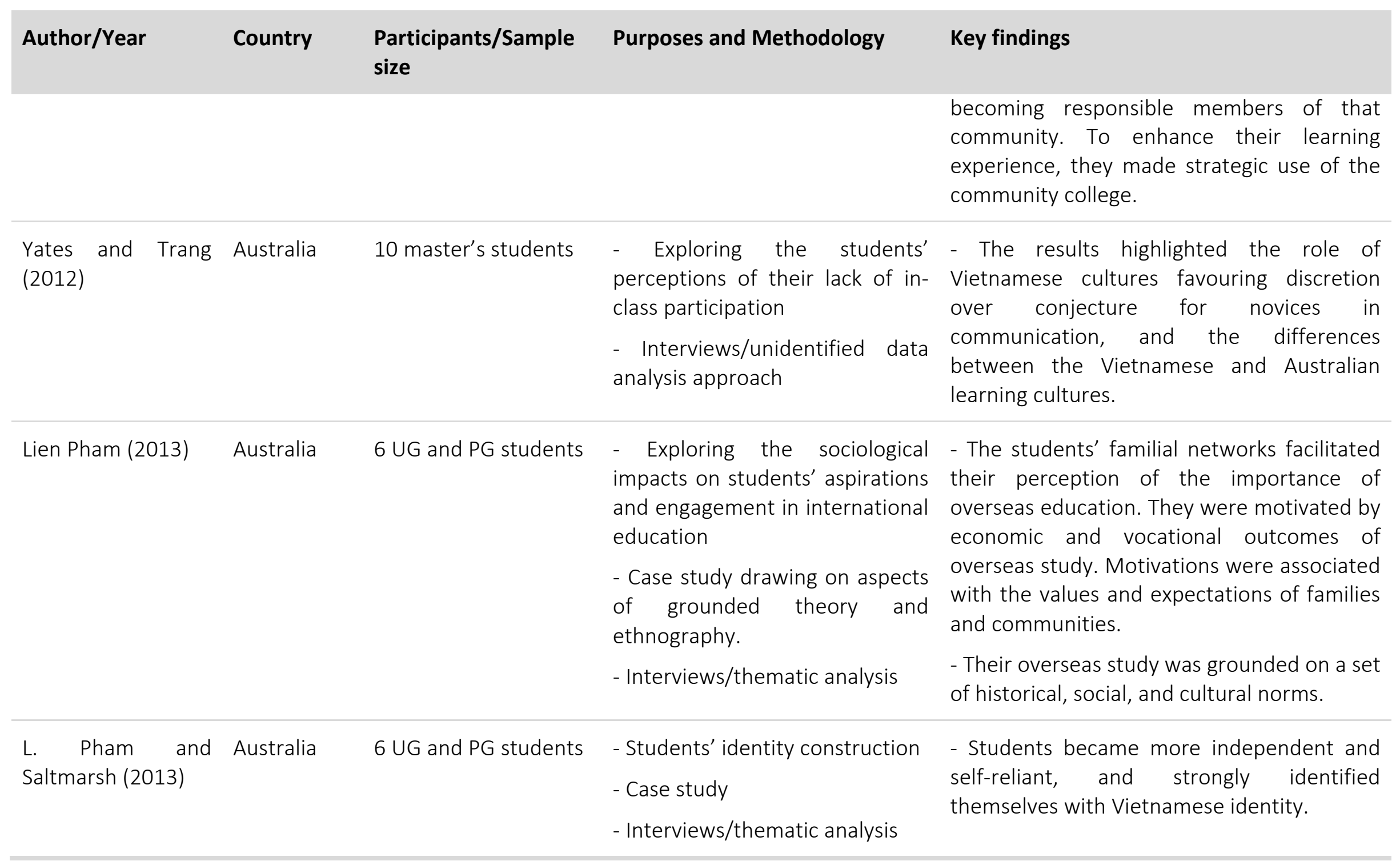




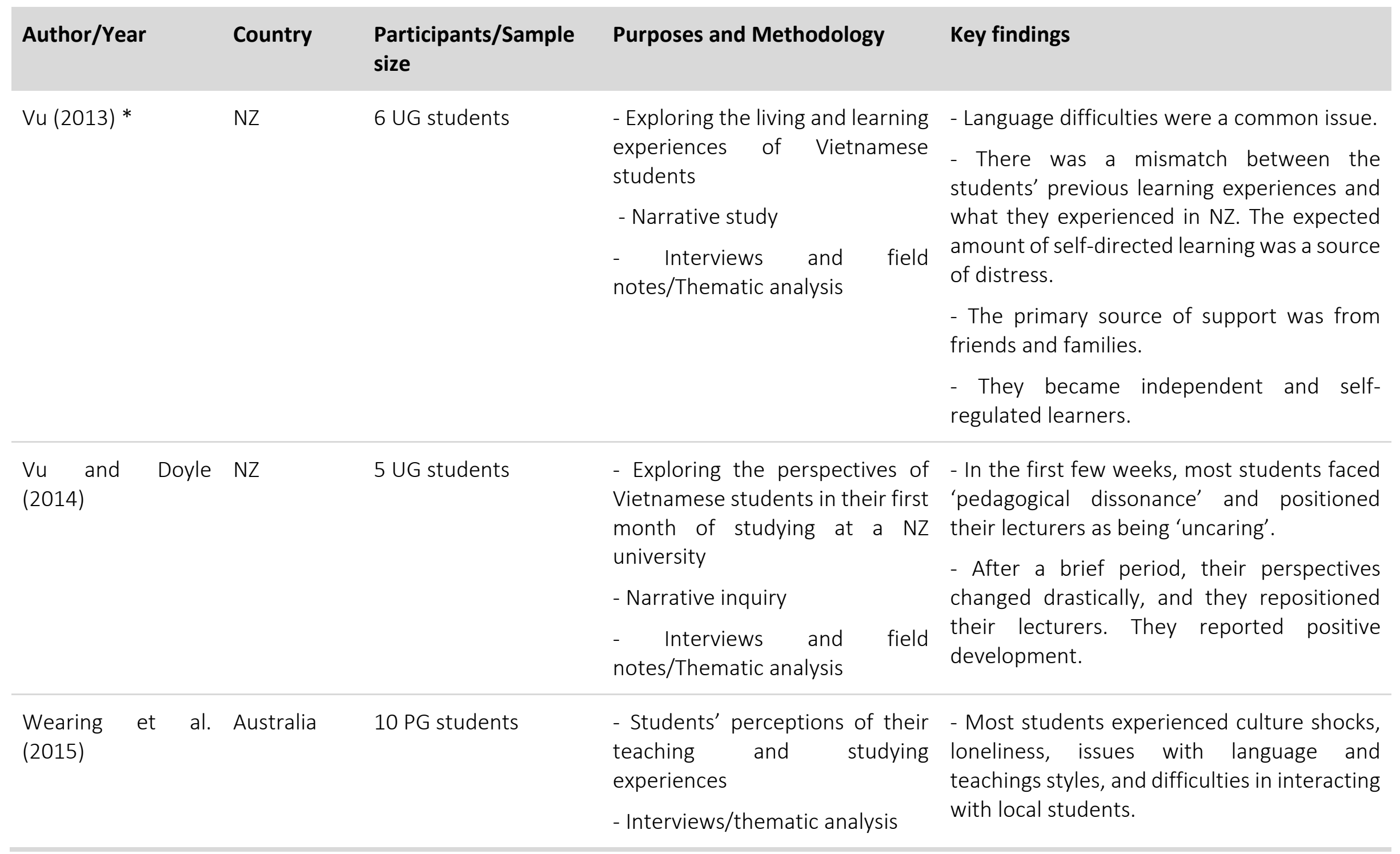




\begin{tabular}{|c|c|c|c|c|}
\hline Author/Year & Country & $\begin{array}{l}\text { Participants/Sample } \\
\text { size }\end{array}$ & Purposes and Methodology & Key findings \\
\hline $\begin{array}{l}\text { T. V. Y. Hoang and } \\
\text { Ma (2016) }\end{array}$ & Australia & 7 PhD students & $\begin{array}{l}\text { - Investigating the students' } \\
\text { experience of writing for } \\
\text { publication in English } \\
\text { - Qualitative/Content analysis }\end{array}$ & $\begin{array}{l}\text { - Students encountered similar difficulties in } \\
\text { scholarly writing to other groups of EAL } \\
\text { (English as an Additional Language) PhD } \\
\text { students (e.g., thinking in Vietnamese, } \\
\text { difficulties in writing sections, and reviewers' } \\
\text { negative comments on their language). } \\
\text { - Students faced different strategies and } \\
\text { challenges (e.g., power inequality between } \\
\text { co-authors, linguistic bias in journal } \\
\text { gatekeeping) from those of students in other } \\
\text { non-English speaking countries. }\end{array}$ \\
\hline L. T. Pham (2016) * & Vietnam & $\begin{array}{l}\text { Returnees with } \\
\text { Bachelor, master's and } \\
\text { PhD degrees from } \\
\text { various countries, such } \\
\text { as Australia, the USA, } \\
\text { the UK, and Singapore } \\
280 \\
\text { respondents survey } \\
48 \text { interviewees }\end{array}$ & $\begin{array}{l}\text { - Exploring the transformative } \\
\text { potential of Vietnamese } \\
\text { overseas-educated returnees in } \\
\text { their local communities } \\
\text { - Mixed methods case study } \\
\text { - Surveys/descriptive statistics } \\
\text { and interviews/thematic analysis }\end{array}$ & $\begin{array}{l}\text { - The findings highlighted the types of } \\
\text { overseas-acquired skills, attributes, } \\
\text { knowledge, and attitudes that the } \\
\text { participants utilised in their workplaces and } \\
\text { communities. }\end{array}$ \\
\hline
\end{tabular}




\begin{tabular}{|c|c|c|c|c|}
\hline Author/Year & Country & $\begin{array}{l}\text { Participants/Sample } \\
\text { size }\end{array}$ & Purposes and Methodology & Key findings \\
\hline $\begin{array}{l}\text { H.-H. Pham and Lai } \\
(2016)\end{array}$ & $\begin{array}{l}15+ \\
\text { countries }\end{array}$ & $\begin{array}{l}252 \text { UG and PG } \\
\text { students }\end{array}$ & $\begin{array}{l}\text { - Exploring the roles of adjusted } \\
\text { expectation, satisfaction, } \\
\text { disconfirmation, and } \\
\text { commitment in student loyalty } \\
\text { - Questionnaires (using a } \\
\text { structure equation model to } \\
\text { analyse data) }\end{array}$ & $\begin{array}{l}\text { - The direct and indirect roles of satisfaction } \\
\text { and commitment in international student } \\
\text { loyalty were confirmed. } \\
\text { - Adjusted expectation mediated the } \\
\text { satisfaction-commitment relationship and } \\
\text { affected their loyalty through commitment. }\end{array}$ \\
\hline T. H. L. Bui (2017) * & UK & 8 PG students & $\begin{array}{l}\text { - Investing students' negotiation } \\
\text { of social and academic identities } \\
\text { - Narrative inquiry } \\
\text { - Interviews/thematic analysis } \\
\text { and positioning analysis }\end{array}$ & $\begin{array}{l}\text { - Identity negotiation involved reflective and } \\
\text { interactive positionings. Most participants } \\
\text { preferred standard English and viewed } \\
\text { themselves as deficient compared to local } \\
\text { and European peers. } \\
\text { - Their transformation in positionings of their } \\
\text { own and others' English was highlighted. }\end{array}$ \\
\hline $\begin{array}{l}\text { Dang and Tran } \\
\text { (2017) }\end{array}$ & Australia & 2 PhD students & $\begin{array}{l}\text { - Investing students' academic } \\
\text { connectedness in the } \\
\text { international education context } \\
\text { - Narrative inquiry } \\
\text { - Interviews/Thematic analysis }\end{array}$ & $\begin{array}{l}\text { - The students' connectedness was built } \\
\text { idiosyncratically and influenced by many } \\
\text { factors (e.g., identities, positions, and } \\
\text { transnational social fields). } \\
\text { - They felt connected to host institutions but } \\
\text { maintained a strong sense of belongingness } \\
\text { to home institutions. }\end{array}$ \\
\hline
\end{tabular}




\begin{tabular}{|c|c|c|c|c|}
\hline Author/Year & Country & $\begin{array}{l}\text { Participants/Sample } \\
\text { size }\end{array}$ & Purposes and Methodology & Key findings \\
\hline $\begin{array}{ll}\text { H. Le, Koo, } & \text { Krambewela, and } \\
\text { Zutshi (2017) } & \end{array}$ & Australia & $\begin{array}{l}\text { Vietnamese students } \\
\text { and Australian staff } \\
\text { members (lecturer, } \\
\text { student advisor and } \\
\text { administrator) } \\
12 \text { UG and PG students } \\
\text { for focus groups } \\
10 \text { students and } 4 \text { staff } \\
\text { members for } \\
\text { interviews }\end{array}$ & $\begin{array}{l}\text { - Examining the students' } \\
\text { experiences with the campus } \\
\text { learning environment } \\
\text { - Focus group and interviews } \\
\text { - Qualitative/Thematic analysis }\end{array}$ & $\begin{array}{l}\text { - There was a divergence of views between } \\
\text { students and staff on teaching and learning. } \\
\text { For example, students expected teachers to } \\
\text { provide answers to all questions while } \\
\text { teachers acted as facilitators of active } \\
\text { learning. Teachers commented that students } \\
\text { were task oriented but had difficulties with } \\
\text { analytical thinking. The differences were } \\
\text { driven by students' expectations of their } \\
\text { learning environment. }\end{array}$ \\
\hline $\begin{array}{l}\text { A. T. Le and LaCost } \\
\text { (2017) }\end{array}$ & Vietnam & $\begin{array}{l}7 \text { (graduates returning } \\
\text { from the USA) }\end{array}$ & $\begin{array}{l}\text { - Exploring the experiences of } \\
\text { students who returned to } \\
\text { Vietnam after graduating from a } \\
\text { USA university } \\
\text { - Interviews/Inductive reasoning } \\
\text { approach }\end{array}$ & $\begin{array}{l}\text { - The students changed considerably after } \\
\text { their time in the USA. Most experienced } \\
\text { unexpected reverse culture shock and had to } \\
\text { make efforts to fit back into the Vietnamese } \\
\text { society. }\end{array}$ \\
\hline A. Pham (2017) & Vietnam & $\begin{array}{l}12 \text { graduates returning } \\
\text { from Australia } \\
12 \text { human resource } \\
\text { managers in Vietnam }\end{array}$ & $\begin{array}{l}\text { - Investigating international } \\
\text { student returnees' re- } \\
\text { engagement with workplaces } \\
\text { and communities in Vietnam } \\
\text { - Case study }\end{array}$ & $\begin{array}{l}\text { - The findings highlighted the participants' } \\
\text { self-formation, re-engagement upon return, } \\
\text { knowledge, skills and working attitudes } \\
\text { acquired through international education, } \\
\text { and reverse culture shock. }\end{array}$ \\
\hline
\end{tabular}




\begin{tabular}{|c|c|c|c|c|}
\hline Author/Year & Country & $\begin{array}{l}\text { Participants/Sample } \\
\text { size }\end{array}$ & Purposes and Methodology & Key findings \\
\hline & & & $\begin{array}{l}\text { - Interviews (unidentified data } \\
\text { analysis approach) }\end{array}$ & $\begin{array}{l}\text { - The student returnees were aware of } \\
\text { challenges in transitioning to work but were } \\
\text { not well-prepared for them. }\end{array}$ \\
\hline $\begin{array}{l}\text { Starks and Nicholas } \\
(2017)\end{array}$ & Vietnam & $\begin{array}{l}3 \text { graduates returning } \\
\text { from Australia }\end{array}$ & $\begin{array}{l}\text { - Exploring how Vietnamese } \\
\text { alumni have changed through } \\
\text { studying abroad and how these } \\
\text { changes are accepted and } \\
\text { contested by their students, } \\
\text { colleagues and the public } \\
\text { - Extended engagement, focus } \\
\text { group interviews (unidentified } \\
\text { data analysis approach) }\end{array}$ & $\begin{array}{l}\text { - The alumni's changes involved self- } \\
\text { presentation (new ways of being, interacting } \\
\text { and talking) and were associated with } \\
\text { practices that the participants viewed as } \\
\text { western. These self-representations were } \\
\text { related to their work context and broader } \\
\text { community and are contested. }\end{array}$ \\
\hline An et al. (2018) & $\begin{array}{l}\text { South Korea } \\
\text { and } \\
\text { Singapore }\end{array}$ & $\begin{array}{l}20 \text { students living in } \\
\text { South Korea and } 25 \\
\text { students living in } \\
\text { Singapore }\end{array}$ & $\begin{array}{l}\text { - Exploring the role of social } \\
\text { media in the daily lives of female } \\
\text { students } \\
\text { - Interviews (unidentified data } \\
\text { analysis approach), focus group } \\
\text { discussions and media diaries } \\
\text { - One week of media monitoring } \\
\text { exercise and one week of media } \\
\text { deprivation exercise. }\end{array}$ & $\begin{array}{l}\text { - Students in South Korea had strong } \\
\text { connection with their co-national friends } \\
\text { because of perceptions of discrimination. } \\
\text { Students in Singapore integrated easily into } \\
\text { the host country. } \\
\text { - Digital media enabled them to sustain their } \\
\text { emotional well-being by helping them } \\
\text { maintain contact with family and friends in } \\
\text { Vietnam. }\end{array}$ \\
\hline
\end{tabular}




\begin{tabular}{|c|c|c|c|c|}
\hline Author/Year & Country & $\begin{array}{l}\text { Participants/Sample } \\
\text { size }\end{array}$ & Purposes and Methodology & Key findings \\
\hline $\begin{array}{l}\text { Huong and Cong } \\
\text { (2018) }\end{array}$ & $\begin{array}{l}\text { The } \\
\text { Philippines }\end{array}$ & 56 PG students & $\begin{array}{l}\text { - Factors influencing the } \\
\text { students' decision to study } \\
\text { abroad and choose the } \\
\text { Philippines as their destination } \\
\text { country } \\
\text { - Mixed methods approach } \\
\text { - Questionnaires/descriptive } \\
\text { statistics and semi-structured } \\
\text { interview/unidentified data } \\
\text { analysis approach }\end{array}$ & $\begin{array}{l}\text { - The study found the motivational factors of } \\
\text { the students' decision to study overseas - } \\
\text { professional development, English } \\
\text { improvement, and better educational } \\
\text { quality, and students' choice of the } \\
\text { Philippines - affordable living costs and } \\
\text { tuition fees, English speaking environment, } \\
\text { convenience of travelling, and proximity to } \\
\text { the home country. }\end{array}$ \\
\hline $\begin{array}{l}\text { B. T. T. Nguyen and } \\
\text { Pennycook (2018) }\end{array}$ & Australia & 24 UG and PG students & $\begin{array}{l}\text { - Exploring students' language, } \\
\text { academic and socio-cultural } \\
\text { experiences } \\
\text { - Interviews/Inductive analysis }\end{array}$ & $\begin{array}{l}\text { - Students struggled to adapt to different } \\
\text { educational norms and supervisors' } \\
\text { expectations. Different strategies were used } \\
\text { to cope with linguistic and other concerns. } \\
\text { - The study highlighted students' agentive } \\
\text { modes of accommodation in academic and } \\
\text { daily life settings. }\end{array}$ \\
\hline L. Nguyen (2018) * & Australia & $38 \mathrm{PhD}$ students & $\begin{array}{l}\text { - Exploring the lived experiences } \\
\text { of students in Australia with a } \\
\text { focus on the reconstruction of } \\
\text { their identity because of their } \\
\text { transnational experiences }\end{array}$ & $\begin{array}{l}\text { - The findings highlighted the students' } \\
\text { identity reconstruction which were } \\
\text { associated with an expanding, } \\
\text { transnationalised social imaginary. The re- } \\
\text { construction of identity included a sense of } \\
\text { agency, a shifting understanding of }\end{array}$ \\
\hline
\end{tabular}




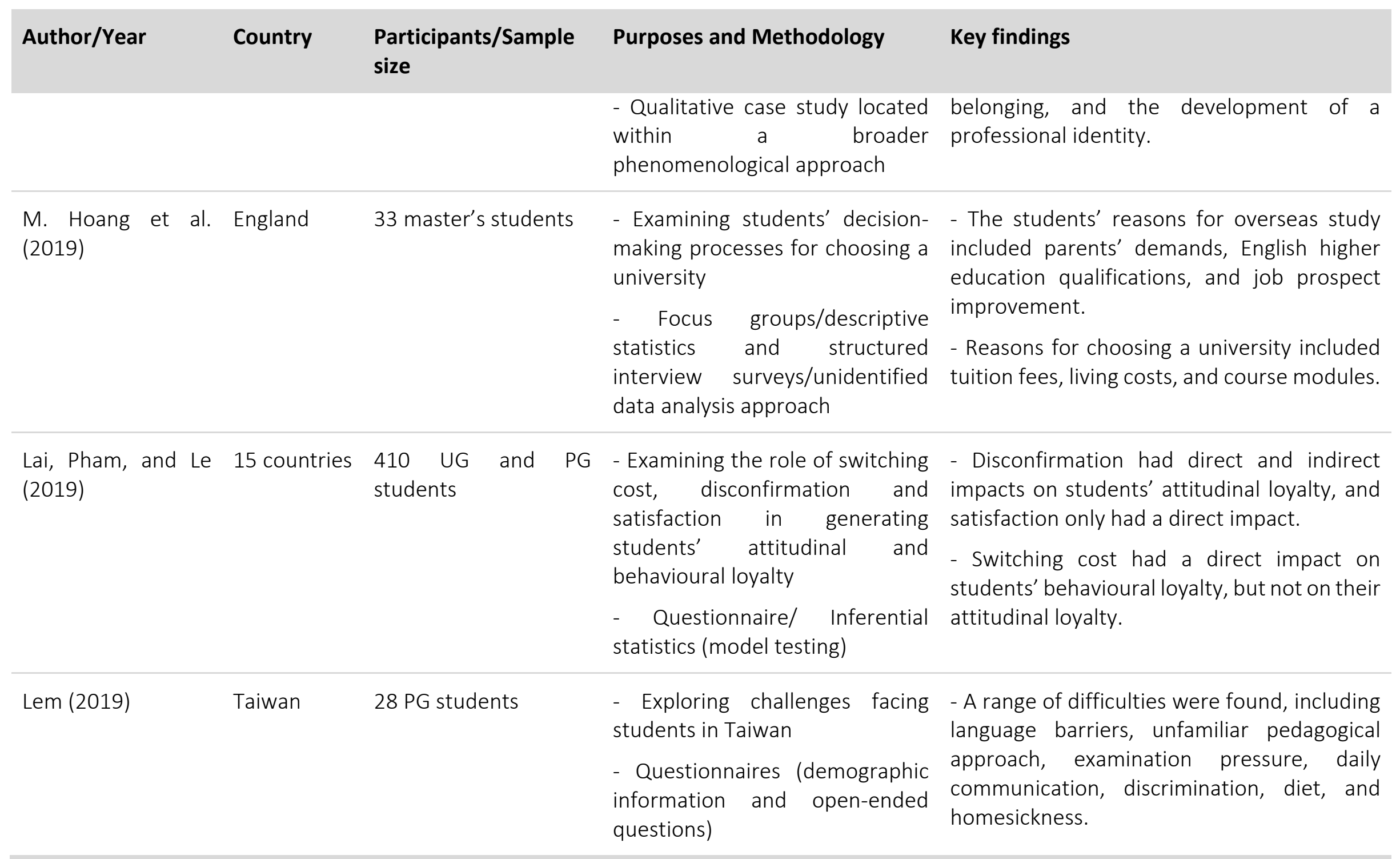




\begin{tabular}{|c|c|c|c|c|}
\hline Author/Year & Country & $\begin{array}{l}\text { Participants/Sample } \\
\text { size }\end{array}$ & Purposes and Methodology & Key findings \\
\hline & & & \multicolumn{2}{|l|}{ - Interviews/Content analysis } \\
\hline $\begin{array}{l}\text { T. T. T. Nguyen } \\
\text { (2019) }\end{array}$ & Taiwan & 36 PG students & $\begin{array}{l}\text { - Examining the language } \\
\text { practices of Vietnamese students } \\
\text { in terms of their social } \\
\text { connectedness across contact } \\
\text { zones } \\
\text { - Interviews/thematic analysis }\end{array}$ & $\begin{array}{l}\text { - The students shaped their social imaginary } \\
\text { of their position through managing their } \\
\text { English and Chinese in communication with } \\
\text { people of various backgrounds. } \\
\text { - Chinese proficiency helped to establish } \\
\text { connectedness with locals. }\end{array}$ \\
\hline T. Nguyen (2019) * & USA & 1 master's student & $\begin{array}{l}\text { - Examining the cross-cultural } \\
\text { adaptation of Vietnamese } \\
\text { students } \\
\text { - Autoethnography }\end{array}$ & $\begin{array}{l}\text { - Stages of her adaptation included the } \\
\text { honeymoon (e.g., pleasant experience in the } \\
\text { classroom), the hostility (e.g., struggling with } \\
\text { cultural differences), and the adjusting (e.g., } \\
\text { negotiating identity, changing behaviours } \\
\text { and 'integrating' with the host culture). These } \\
\text { stages did not occur step by step. } \\
\text { - Challenges were related to the transition to } \\
\text { higher education level, differences in cultural } \\
\text { background, language barrier, and balancing } \\
\text { between the Vietnamese } \\
\text { background/identity and the American } \\
\text { cultural environment. }\end{array}$ \\
\hline
\end{tabular}




\begin{tabular}{|c|c|c|c|c|}
\hline Author/Year & Country & $\begin{array}{l}\text { Participants/Sample } \\
\text { size }\end{array}$ & Purposes and Methodology & Key findings \\
\hline $\begin{array}{l}\text { Q. Nguyen and } \\
\text { Buckingham (2019) }\end{array}$ & NZ & 7 master's students & $\begin{array}{l}\text { - Investigating the students' } \\
\text { approach to using sources in } \\
\text { assignments } \\
\text { - Interviews/thematic analysis } \\
\text { - Students' essays and } \\
\text { assignment guidelines }\end{array}$ & $\begin{array}{l}\text { - The students' overall above-average } \\
\text { academic ability, mature-student status, and } \\
\text { prior writing experience contributed to their } \\
\text { successful performance in their assignments. }\end{array}$ \\
\hline $\begin{array}{l}\text { H.-H. Pham et al. } \\
\text { (2019) }\end{array}$ & $\begin{array}{l}\text { Over } 15 \\
\text { countries }\end{array}$ & $\begin{array}{l}410 \text { UG and PG } \\
\text { students }\end{array}$ & $\begin{array}{l}\text { - Exploring the role of subjective } \\
\text { task value in forming satisfaction } \\
\text { and loyalty of international } \\
\text { students } \\
\text { - Questionnaire/Structural } \\
\text { equation model }\end{array}$ & $\begin{array}{l}\text { - Satisfaction and disconfirmation served as } \\
\text { direct and indirect antecedents of student } \\
\text { loyalty. Intrinsic value directly and indirectly } \\
\text { affected loyalty through satisfaction and } \\
\text { disconfirmation which were mediators. } \\
\text { Attainment value had a slight impact and } \\
\text { utility value had no impact on loyalty. }\end{array}$ \\
\hline L. H. N. Tran (2019) & 17 countries & $\begin{array}{l}368 \text { prospective and } \\
\text { current international } \\
\text { students }\end{array}$ & $\begin{array}{l}\text { - Investigating students' } \\
\text { motivations to study overseas } \\
\text { and their immigration intentions } \\
\text { - Questionnaire/Descriptive } \\
\text { statistics } \\
\text { - Interviews (unidentified data } \\
\text { analysis approach) }\end{array}$ & $\begin{array}{l}\text { - The results revealed } 12 \text { push and pull factors } \\
\text { that motivated the students to study } \\
\text { overseas and } 18 \text { factors that were associated } \\
\text { with their immigration intentions. } \\
\text { - Their personal attachment to the home } \\
\text { country (Vietnam) and perceived adaptability } \\
\text { to a host country affected their immigration } \\
\text { intentions. }\end{array}$ \\
\hline
\end{tabular}




\begin{tabular}{|c|c|c|c|c|}
\hline Author/Year & Country & $\begin{array}{l}\text { Participants/Sample } \\
\text { size }\end{array}$ & Purposes and Methodology & Key findings \\
\hline T. A. Bui (2021) & Canada & 1 PhD student & $\begin{array}{l}\text { The author examines her } \\
\text { experience as an international } \\
\text { doctoral student transitioning to } \\
\text { a new culture } \\
\text { - Self-reflective study }\end{array}$ & $\begin{array}{l}\text { - The findings revealed conflicts and tensions } \\
\text { emerging through various dialectical } \\
\text { processes, highlighting a vulnerable self and } \\
\text { profound changes when the author engaged } \\
\text { in transnational space. }\end{array}$ \\
\hline $\begin{array}{l}\text { M. N. Nguyen and } \\
\text { Robertson (2020) }\end{array}$ & Australia & $\begin{array}{l}6 \text { recent doctoral } \\
\text { graduates }\end{array}$ & $\begin{array}{l}\text { - Investigating students' accounts } \\
\text { of their candidature at a } \\
\text { university, focusing on how they } \\
\text { enacted agency } \\
\text { - Case study } \\
\text { - Interviews/thematic analysis }\end{array}$ & $\begin{array}{l}\text { - The findings highlighted the students' } \\
\text { agency. They had a high level of agency, } \\
\text { which was shown in how they dealt with } \\
\text { language in academic settings, using soft } \\
\text { strategies, transforming into a more } \\
\text { confident self, and mobilising their families } \\
\text { and maturated networks. }\end{array}$ \\
\hline $\begin{array}{l}\text { N. C. Pham and Shi } \\
(2020)\end{array}$ & USA & 20 UG and PG students & $\begin{array}{l}\text { - Exploring how the COVID-19 } \\
\text { pandemic impacts on } \\
\text { Vietnamese students' mental } \\
\text { distress } \\
\text { - Interviews/narrative textual } \\
\text { analysis }\end{array}$ & $\begin{array}{l}\text { - Factors that contributed to the students' } \\
\text { mental distress included limited access to on- } \\
\text { campus facilities and public services, risk of } \\
\text { infection, isolation during the lockdown, and } \\
\text { inability to return to Vietnam. }\end{array}$ \\
\hline $\begin{array}{l}\text { N. T. T. Nguyen } \\
(2020)\end{array}$ & USA & 1 PhD student & $\begin{array}{l}\text { - Exploring the experience of } \\
\text { Vietnamese international female } \\
\text { PG students }\end{array}$ & $\begin{array}{l}\text { - The student encountered stereotypes and } \\
\text { language difficulties. Her personality (e.g., } \\
\text { timid and unsociable manners) contributed }\end{array}$ \\
\hline
\end{tabular}




\begin{tabular}{|c|c|c|c|c|}
\hline \multirow[t]{3}{*}{ Author/Year } & Country & $\begin{array}{l}\text { Participants/Sample } \\
\text { size }\end{array}$ & Purposes and Methodology & Key findings \\
\hline & & & - Interviews/narrative enquiry & $\begin{array}{l}\text { to her challenges. She viewed herself as an } \\
\text { outsider in her initial university. }\end{array}$ \\
\hline & & & & $\begin{array}{l}\text { - She sought to cope with challenges through } \\
\text { being self-reliant and adjusting herself to } \\
\text { academic norms. She lacked definite } \\
\text { resources, for example, voices and women's } \\
\text { collective strengths. }\end{array}$ \\
\hline \multirow[t]{4}{*}{$\begin{array}{l}\text { Robertson and } \\
\text { Nguyen (2020) }\end{array}$} & Australia & $\begin{array}{l}6 \text { recent doctoral } \\
\text { graduates }\end{array}$ & $\begin{array}{l}\text { - Exploring students' identity } \\
\text { development after their } \\
\text { candidature at a university, }\end{array}$ & $\begin{array}{l}\text { - Cultural differences of power relationships } \\
\text { contributed to the challenges of identity } \\
\text { development. }\end{array}$ \\
\hline & & & $\begin{array}{l}\text { focusing on how they } \\
\text { empowered themselves and } \\
\text { formed their academic identities }\end{array}$ & $\begin{array}{l}\text { - The participants found it hard to deal with } \\
\text { their supervisory relationships at some stages } \\
\text { of their candidature. They tried to develop }\end{array}$ \\
\hline & & & - Case study & their new doctoral identity in different ways. \\
\hline & & & - Interviews/thematic analysis & $\begin{array}{l}\text { - Gender differences were seen in their } \\
\text { interactions with their supervisors. }\end{array}$ \\
\hline
\end{tabular}

UG: Undergraduate

PG: Postgraduate

NZ: New Zealand

An asterisk $\left({ }^{*}\right)$ indicates the source is a thesis. 